Supporting information for

\title{
An Isoxazole Strategy for the Synthesis of Fully Substituted Nicotinates
}

Ekaterina E. Galenko, Mariya A. Kryukova, Mikhail S. Novikov, Alexander F. Khlebnikov*

St. Petersburg State University, Institute of Chemistry, 7/9 Universitetskaya nab., St. Petersburg, 199034, Russia

E-mail: a.khlebnokov@spbu.ru

Table of Contents

\begin{tabular}{|l|l|}
\hline X-Ray Diffraction Experiments for 4i & S2 \\
\hline X-Ray Diffraction Experiments for 7b & S7 \\
\hline X-Ray Diffraction Experiments for 7c & S13 \\
\hline${ }^{1}$ H NMR Spectrum of Compound 11b & S19 \\
\hline NMR Spectra of Compounds 1 & S20 \\
\hline NMR Spectra of Compound 2d & S32 \\
\hline NMR Spectra of Compounds 4i, 4'i & S35 \\
\hline NMR Spectra of Compounds 7 & S 41 \\
\hline NMR Spectra of Compound 8 & S86 \\
\hline NMR Spectra of Compounds 9,10 & S89 \\
\hline
\end{tabular}




\section{X-RAY DIFFRACTION EXPERIMENTS}

Crystal structures of $\mathbf{4 i}, \mathbf{7 b}$ and $\mathbf{7} \mathbf{c}$ were determined by single crystal X-ray diffraction analysis. Suitable crystals were selected and fixed on micro-mounts and the diffraction data were collected on a HyPix diffractometer. The crystals of $\mathbf{4 i}, \mathbf{7 b}$ and $\mathbf{7 c}$ were measured at a temperature of $100(2) \mathrm{K}$, using monochromated $\mathrm{CuK} \alpha$ radiation. The unit cell parameters and refinement characteristics of the crystal structures of 4i, 7b and 7c are given below. Using Olex2 [1], the structures were solved with the ShelXT [2] structure solution program using Intrinsic Phasing and refined with the ShelXL [3] refinement package using Least Squares minimization.

References

1. Dolomanov, O.V.; Bourhis, L.J.; Gildea, R.J; Howard, J.A.K.; Puschmann, H. J. Appl. Cryst. 2009, 42, 339.

2. Sheldrick, G.M. Acta Cryst. 2015, A71,

3. 3. Sheldrick, G.M.Acta Cryst. 2015, C71, 3.

\section{2-((5-Methoxy-3-phenylisoxazol-4-yl)(4-nitrophenyl)methyl)-3,4-dihydronaphthalen-1(2H)-one}

Single crystals of $\mathbf{4 i}$ were obtained by slow evaporation of dichloromethane solution at room temperature. (CCDC 2053525).

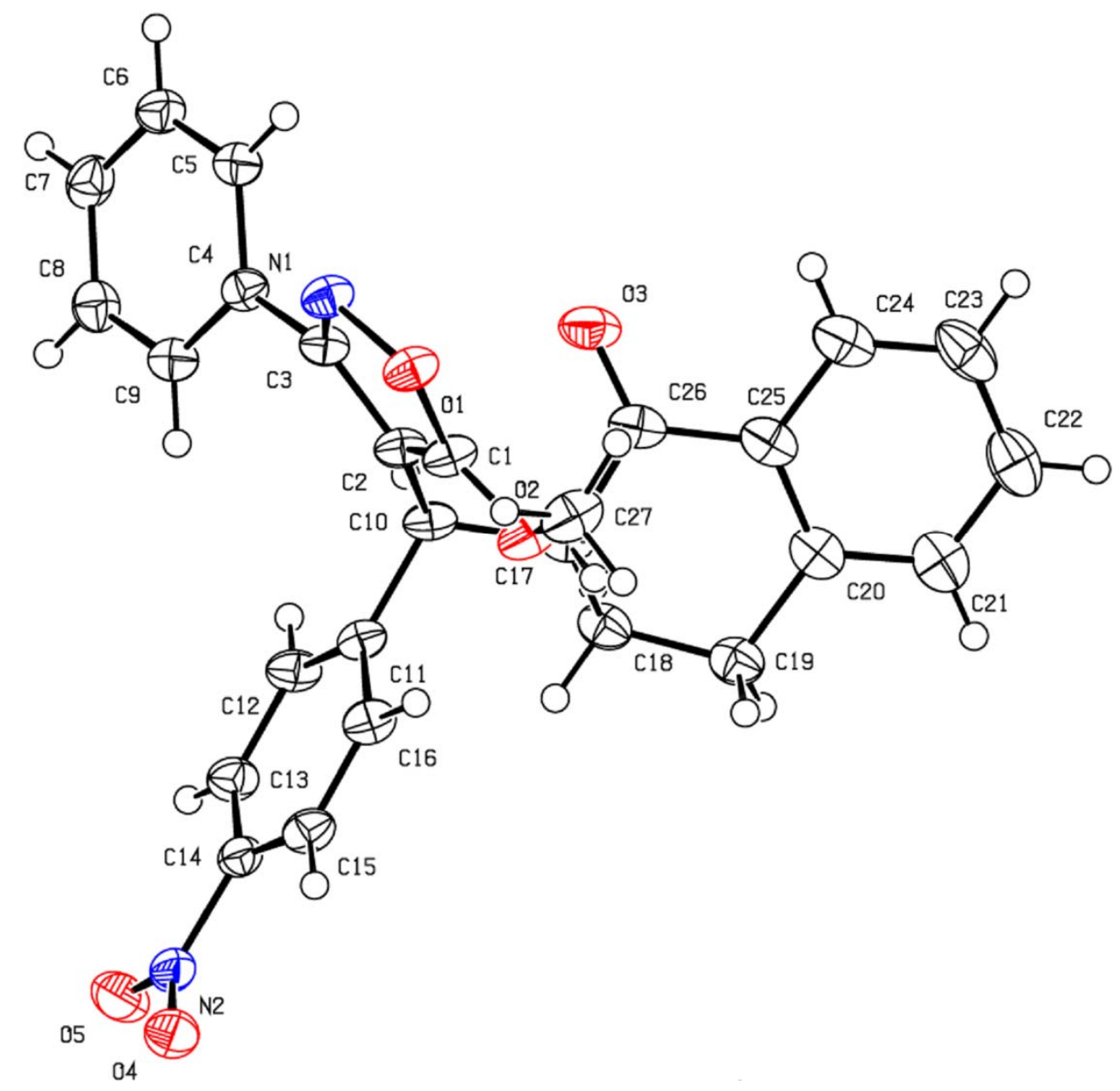

Molecular structure of compound $\mathbf{4 i}$, displacement parameters are drawn at 50\% probability level. 
Table S1. Crystal data and structure refinement for $4 i$.

\begin{tabular}{|c|c|}
\hline Identification code & 4i (17626_CAT16.56a) \\
\hline Empirical formula & $\mathrm{C}_{27} \mathrm{H}_{22} \mathrm{~N}_{2} \overline{\mathrm{O}}_{5}$ \\
\hline Formula weight & 454.46 \\
\hline Temperature/K & $100.00(10)$ \\
\hline Crystal system & monoclinic \\
\hline Space group & $\mathrm{P} 2{ }_{1} / \mathrm{c}$ \\
\hline $\mathrm{a} / \AA$ & $9.9953(2)$ \\
\hline $\mathrm{b} / \AA$ & $14.5820(2)$ \\
\hline $\mathrm{c} / \AA$ & $15.5765(2)$ \\
\hline$\alpha /^{\circ}$ & 90 \\
\hline$\beta /{ }^{\circ}$ & $99.4140(10)$ \\
\hline$\gamma /{ }^{\circ}$ & 90 \\
\hline Volume $/ \AA^{3}$ & $2239.72(6)$ \\
\hline Z & 4 \\
\hline$\rho_{\text {calc }} \mathrm{g} / \mathrm{cm}^{3}$ & 1.348 \\
\hline$\mu / \mathrm{mm}^{-1}$ & 0.769 \\
\hline $\mathrm{F}(000)$ & 952.0 \\
\hline Crystal size $/ \mathrm{mm}^{3}$ & $0.15 \times 0.13 \times 0.10$ \\
\hline Radiation & $\mathrm{CuK} \alpha(\lambda=1.54184)$ \\
\hline $2 \Theta$ range for data collection ${ }^{\circ}$ & 8.36 to 140.924 \\
\hline Index ranges & $-10 \leq \mathrm{h} \leq 12,-17 \leq \mathrm{k} \leq 17,-18 \leq 1 \leq 19$ \\
\hline Reflections collected & 13848 \\
\hline Independent reflections & $4274\left[\mathrm{R}_{\text {int }}=0.0326, \mathrm{R}_{\text {sigma }}=0.0335\right]$ \\
\hline Data/restraints/parameters & $4274 / 0 / 308$ \\
\hline Goodness-of-fit on $\mathrm{F}^{2}$ & 1.039 \\
\hline Final $\mathrm{R}$ indexes $[\mathrm{I}>=2 \sigma(\mathrm{I})]$ & $\mathrm{R}_{1}=0.0384, \mathrm{wR}_{2}=0.0994$ \\
\hline Final $\mathrm{R}$ indexes [all data] & $\mathrm{R}_{1}=0.0446, \mathrm{wR}_{2}=0.1042$ \\
\hline Largest diff. peak/hole / e $\AA^{-3}$ & $0.14 /-0.23$ \\
\hline
\end{tabular}

Table S2. Fractional Atomic Coordinates $\left(\times 10^{4}\right)$ and Equivalent Isotropic Displacement Parameters $\left(\AA^{2} \times 10^{3}\right)$ for $4 i$. $U_{e q}$ is defined as $1 / 3$ of of the trace of the orthogonalised $U_{I J}$ tensor.

\begin{tabular}{|l|l|l|l|l|}
\hline \multicolumn{1}{|c|}{ Atom } & \multicolumn{1}{|c|}{$\boldsymbol{1}$} & \multicolumn{1}{c|}{$\boldsymbol{y}$} & \multicolumn{1}{c|}{$\mathbf{z}$} & \multicolumn{1}{c|}{$\mathbf{U}(\mathbf{e q})$} \\
\hline $\mathrm{O}_{1}$ & $8730.5(9)$ & $602.1(7)$ & $5340.2(6)$ & $28.8(2)$ \\
\hline $\mathrm{O}_{2}$ & $8905.9(10)$ & $1852.6(7)$ & $4471.6(6)$ & $33.9(2)$ \\
\hline $\mathrm{O}_{4}$ & $3219.1(12)$ & $2091.2(8)$ & $1040.3(6)$ & $39.1(3)$ \\
\hline $\mathrm{O}_{3}$ & $7702.0(11)$ & $2926.4(8)$ & $6627.6(6)$ & $38.1(3)$ \\
\hline $\mathrm{O}_{5}$ & $1508.7(11)$ & $2519.1(9)$ & $1632.8(7)$ & $42.6(3)$ \\
\hline $\mathrm{N}_{1}$ & $7796.2(1)$ & $233.5(8)$ & $5850.8(7)$ & $28.2(3)$ \\
\hline $\mathrm{N}_{2}$ & $2710.9(13)$ & $2324.1(8)$ & $1676.0(7)$ & $30.4(3)$ \\
\hline $\mathrm{C}_{11}$ & $5238.8(13)$ & $2405.0(9)$ & $4133.5(8)$ & $24.3(3)$ \\
\hline $\mathrm{C}_{10}$ & $6140.8(13)$ & $2440.8(9)$ & $5024.8(8)$ & $25.3(3)$ \\
\hline $\mathrm{C}_{14}$ & $3599.5(14)$ & $2364.1(9)$ & $2522.7(8)$ & $25.6(3)$ \\
\hline $\mathrm{C}_{2}$ & $6984.3(14)$ & $1584.6(9)$ & $5221.0(8)$ & $26.0(3)$ \\
\hline $\mathrm{C}_{12}$ & $3851.6(14)$ & $2556.4(10)$ & $4057.0(8)$ & $28.6(3)$ \\
\hline $\mathrm{C}_{16}$ & $5795.3(14)$ & $2249.4(9)$ & $3378.8(8)$ & $28.0(3)$ \\
\hline $\mathrm{C}_{15}$ & $4975.1(15)$ & $2232.2(9)$ & $2569.7(8)$ & $28.6(3)$ \\
\hline $\mathrm{C}_{3}$ & $6788.6(13)$ & $830.4(9)$ & $5769.2(8)$ & $25.7(3)$ \\
\hline $\mathrm{C}_{1}$ & $8200.5(14)$ & $1387.2(9)$ & $4985.4(8)$ & $28.0(3)$ \\
\hline $\mathrm{C}_{4}$ & $5646.2(14)$ & $636.7(9)$ & $6234.3(8)$ & $25.2(3)$ \\
\hline $\mathrm{C}_{9}$ & $4309.4(14)$ & $763.4(9)$ & $5828.6(8)$ & $28.0(3)$ \\
\hline $\mathrm{C}_{26}$ & $7785.5(13)$ & $3456.7(10)$ & $6034.6(8)$ & $29.2(3)$ \\
\hline
\end{tabular}




\begin{tabular}{|l|l|l|l|l|}
\hline $\mathrm{C}_{17}$ & $7045.3(13)$ & $3303.9(9)$ & $5110.4(8)$ & $25.4(3)$ \\
\hline $\mathrm{C}_{25}$ & $8570.9(14)$ & $4328.2(11)$ & $6179.7(8)$ & $31.1(3)$ \\
\hline $\mathrm{C}_{5}$ & $5892.3(15)$ & $287.6(10)$ & $7082.1(8)$ & $29.7(3)$ \\
\hline $\mathrm{C}_{13}$ & $3019.0(14)$ & $2542.3(10)$ & $3252.9(8)$ & $29.3(3)$ \\
\hline $\mathrm{C}_{8}$ & $3243.6(14)$ & $551.2(10)$ & $6261.3(9)$ & $30.9(3)$ \\
\hline $\mathrm{C}_{20}$ & $8357.9(14)$ & $5050.1(10)$ & $5578.4(9)$ & $30.9(3)$ \\
\hline $\mathrm{C}_{18}$ & $6293.0(14)$ & $4201.4(9)$ & $4836.9(9)$ & $29.6(3)$ \\
\hline $\mathrm{C}_{7}$ & $3495.2(15)$ & $215.6(10)$ & $7106.8(9)$ & $32.5(3)$ \\
\hline $\mathrm{C}_{6}$ & $4822.3(15)$ & $80.7(10)$ & $7511.1(9)$ & $31.9(3)$ \\
\hline $\mathrm{C}_{19}$ & $7309.9(15)$ & $4965.4(10)$ & $4765.9(9)$ & $32.3(3)$ \\
\hline $\mathrm{C}_{24}$ & $9506.3(14)$ & $4432.4(12)$ & $6948.5(9)$ & $38.3(4)$ \\
\hline $\mathrm{C}_{27}$ & $10219.0(15)$ & $1506.6(11)$ & $4384.1(10)$ & $37.7(3)$ \\
\hline $\mathrm{C}_{21}$ & $9112.0(15)$ & $5854.5(11)$ & $5750.1(10)$ & $38.3(3)$ \\
\hline $\mathrm{C}_{22}$ & $10030.3(15)$ & $5953.0(12)$ & $6513.2(11)$ & $43.1(4)$ \\
\hline $\mathrm{C}_{23}$ & $10220.7(15)$ & $5244.2(14)$ & $7117.9(10)$ & $44.4(4)$ \\
\hline
\end{tabular}

Table S3. Anisotropic Displacement Parameters $\left(\AA^{2} \times 10^{3}\right)$ for $4 \mathrm{i}$. The Anisotropic displacement factor exponent takes the form: $-2 \pi^{2}\left[h^{2} a *^{2} U_{11}+2 h k a * b * U_{12}+\ldots\right]$.

\begin{tabular}{|c|c|c|c|c|c|c|}
\hline Atom & $\mathrm{U}_{11}$ & $\mathbf{U}_{22}$ & $\mathbf{U}_{33}$ & $\mathbf{U}_{23}$ & $\mathbf{U}_{13}$ & $\mathbf{U}_{12}$ \\
\hline $\mathrm{O}_{1}$ & $27.7(5)$ & $29.7(5)$ & $30.9(5)$ & $7.9(4)$ & $10.0(4)$ & $8.9(4)$ \\
\hline $\mathrm{O}_{2}$ & $31.0(5)$ & $34.3(5)$ & $40.6(5)$ & $12.7(4)$ & 18.7(4) & $12.6(4)$ \\
\hline $\mathrm{O}_{4}$ & $54.1(7)$ & $43.1(6)$ & $21.8(5)$ & $-6.0(4)$ & $11.3(4)$ & $-9.2(5)$ \\
\hline $\mathrm{O}_{3}$ & $34.5(6)$ & $55.7(7)$ & $24.3(5)$ & $8.3(4)$ & $5.1(4)$ & $3.2(5)$ \\
\hline $\mathrm{O}_{5}$ & $34.3(6)$ & $63.3(8)$ & $28.7(5)$ & $1.0(5)$ & $0.8(4)$ & $-6.1(5)$ \\
\hline $\mathrm{N}_{1}$ & $28.1(6)$ & $31.4(6)$ & $26.5(5)$ & $6.2(4)$ & $8.9(4)$ & $5.0(5)$ \\
\hline $\mathrm{N}_{2}$ & $39.7(7)$ & $30.9(6)$ & $21.4(5)$ & $0.1(4)$ & $7.0(5)$ & $-8.2(5)$ \\
\hline $\mathrm{C}_{11}$ & $28.7(7)$ & $23.8(6)$ & $21.8(6)$ & $3.3(5)$ & $8.0(5)$ & $4.5(5)$ \\
\hline $\mathrm{C}_{10}$ & $26.8(7)$ & $29.4(7)$ & $21.2(6)$ & $3.9(5)$ & $8.2(5)$ & $8.6(5)$ \\
\hline $\mathrm{C}_{14}$ & $33.9(7)$ & $22.9(6)$ & $20.6(6)$ & $0.9(5)$ & $6.1(5)$ & $-2.5(5)$ \\
\hline $\mathrm{C}_{2}$ & $26.8(7)$ & $30.3(7)$ & $22.1(6)$ & $4.8(5)$ & $7.4(5)$ & $6.8(5)$ \\
\hline $\mathrm{C}_{12}$ & $29.5(7)$ & $38.0(7)$ & $20.3(6)$ & $1.3(5)$ & $10.1(5)$ & $5.4(6)$ \\
\hline $\mathrm{C}_{16}$ & $28.9(7)$ & $30.2(7)$ & $27.0(6)$ & $2.0(5)$ & $11.0(5)$ & $5.9(5)$ \\
\hline $\mathrm{C}_{15}$ & $36.9(7)$ & $29.1(7)$ & $22.8(6)$ & $0.0(5)$ & $13.4(5)$ & $2.8(6)$ \\
\hline $\mathrm{C}_{3}$ & $26.5(6)$ & $29.7(7)$ & $21.0(6)$ & $1.4(5)$ & $4.4(5)$ & $4.2(5)$ \\
\hline $\mathrm{C}_{1}$ & $30.5(7)$ & $28.3(7)$ & $26.7(6)$ & $7.0(5)$ & $9.3(5)$ & $7.6(5)$ \\
\hline $\mathrm{C}_{4}$ & $28.7(7)$ & $24.5(6)$ & $23.2(6)$ & $0.0(5)$ & $6.7(5)$ & $2.2(5)$ \\
\hline $\mathrm{C}_{9}$ & $30.8(7)$ & $29.2(7)$ & $23.9(6)$ & $1.1(5)$ & $4.5(5)$ & $3.9(5)$ \\
\hline $\mathrm{C}_{26}$ & $24.0(6)$ & $41.5(8)$ & $23.0(6)$ & $1.5(5)$ & $7.0(5)$ & $10.2(6)$ \\
\hline $\mathrm{C}_{17}$ & $25.7(6)$ & $30.3(7)$ & $20.8(6)$ & $1.9(5)$ & $5.5(5)$ & $9.0(5)$ \\
\hline $\mathrm{C}_{25}$ & $23.2(6)$ & $45.7(8)$ & $25.3(6)$ & $-5.5(6)$ & $6.8(5)$ & $7.7(6)$ \\
\hline $\mathrm{C}_{5}$ & $31.5(7)$ & $32.9(7)$ & $24.2(6)$ & $2.2(5)$ & $2.9(5)$ & $-1.2(6)$ \\
\hline $\mathrm{C}_{13}$ & $27.5(7)$ & $37.1(7)$ & $24.5(6)$ & $3.0(5)$ & $7.8(5)$ & $1.7(6)$ \\
\hline $\mathrm{C}_{8}$ & 26.9(7) & $30.6(7)$ & $35.2(7)$ & $-1.4(5)$ & $4.9(5)$ & $0.9(5)$ \\
\hline $\mathrm{C}_{20}$ & $26.0(7)$ & $35.6(7)$ & $31.5(7)$ & $-6.6(6)$ & $5.9(5)$ & $8.5(6)$ \\
\hline $\mathrm{C}_{18}$ & $29.8(7)$ & $29.1(7)$ & $28.3(6)$ & $-1.7(5)$ & $-0.1(5)$ & $8.9(6)$ \\
\hline $\mathrm{C}_{7}$ & $33.9(7)$ & $33.8(7)$ & $32.8(7)$ & $-2.0(6)$ & $13.9(6)$ & $-4.9(6)$ \\
\hline $\mathrm{C}_{6}$ & $39.9(8)$ & $33.2(7)$ & $23.0(6)$ & $1.1(5)$ & $6.7(5)$ & $-4.3(6)$ \\
\hline $\mathrm{C}_{19}$ & $34.1(7)$ & $29.9(7)$ & $31.5(7)$ & $-0.1(5)$ & $1.5(6)$ & $7.9(6)$ \\
\hline $\mathrm{C}_{24}$ & $25.7(7)$ & $61.9(10)$ & $27.8(7)$ & $-2.3(7)$ & $5.6(5)$ & $5.5(7)$ \\
\hline $\mathrm{C}_{27}$ & $28.0(7)$ & $42.6(8)$ & $45.9(8)$ & $13.5(7)$ & 16.1(6) & $11.2(6)$ \\
\hline $\mathrm{C}_{21}$ & $29.9(7)$ & $40.6(8)$ & $44.2(8)$ & $-7.7(6)$ & $5.7(6)$ & $7.5(6)$ \\
\hline $\mathrm{C}_{22}$ & $27.1(7)$ & $51.3(10)$ & $50.2(9)$ & $-16.0(8)$ & $4.3(6)$ & $1.7(7)$ \\
\hline $\mathrm{C}_{23}$ & $23.1(7)$ & $72.3(12)$ & $36.9(8)$ & $-14.9(8)$ & $2.1(6)$ & $1.8(7)$ \\
\hline
\end{tabular}


Table S4. Bond Lengths for $4 i$.

\begin{tabular}{|c|c|c|c|c|c|}
\hline Atom & Atom & Length/Å & Atom & Atom & Length/Å \\
\hline $\mathrm{O}_{1}$ & $\mathrm{~N}_{1}$ & $1.4272(14)$ & $\mathrm{C}_{16}$ & $\mathrm{C}_{15}$ & $1.387(2)$ \\
\hline $\mathrm{O}_{1}$ & $\mathrm{C}_{1}$ & $1.3420(16)$ & $\mathrm{C}_{3}$ & $\mathrm{C}_{4}$ & $1.4761(18)$ \\
\hline $\mathrm{O}_{2}$ & $\mathrm{C}_{1}$ & $1.3352(16)$ & $\mathrm{C}_{4}$ & $\mathrm{C}_{9}$ & $1.3938(19)$ \\
\hline $\mathrm{O}_{2}$ & $\mathrm{C}_{27}$ & $1.4333(16)$ & $\mathrm{C}_{4}$ & $\mathrm{C}_{5}$ & $1.3991(18)$ \\
\hline $\mathrm{O}_{4}$ & $\mathrm{~N}_{2}$ & $1.2322(15)$ & $\mathrm{C}_{9}$ & $\mathrm{C}_{8}$ & $1.386(2)$ \\
\hline $\mathrm{O}_{3}$ & $\mathrm{C}_{26}$ & $1.2179(18)$ & $\mathrm{C}_{26}$ & $\mathrm{C}_{17}$ & $1.5234(17)$ \\
\hline $\mathrm{O}_{5}$ & $\mathrm{~N}_{2}$ & $1.2260(17)$ & $\mathrm{C}_{26}$ & $\mathrm{C}_{25}$ & $1.491(2)$ \\
\hline $\mathrm{N}_{1}$ & $\mathrm{C}_{3}$ & $1.3215(17)$ & $\mathrm{C}_{17}$ & $\mathrm{C}_{18}$ & $1.5346(18)$ \\
\hline $\mathrm{N}_{2}$ & $\mathrm{C}_{14}$ & $1.4658(17)$ & $\mathrm{C}_{25}$ & $\mathrm{C}_{20}$ & $1.402(2)$ \\
\hline $\mathrm{C}_{11}$ & $\mathrm{C}_{10}$ & $1.5276(17)$ & $\mathrm{C}_{25}$ & $\mathrm{C}_{24}$ & $1.402(2)$ \\
\hline $\mathrm{C}_{11}$ & $\mathrm{C}_{12}$ & $1.3898(19)$ & $\mathrm{C}_{5}$ & $\mathrm{C}_{6}$ & $1.3839(19)$ \\
\hline $\mathrm{C}_{11}$ & $\mathrm{C}_{16}$ & $1.3988(17)$ & $\mathrm{C}_{8}$ & $\mathrm{C}_{7}$ & $1.389(2)$ \\
\hline $\mathrm{C}_{10}$ & $\mathrm{C}_{2}$ & $1.5097(17)$ & $\mathrm{C}_{20}$ & $\mathrm{C}_{19}$ & $1.5101(19)$ \\
\hline $\mathrm{C}_{10}$ & $\mathrm{C}_{17}$ & $1.5427(19)$ & $\mathrm{C}_{20}$ & $\mathrm{C}_{21}$ & $1.396(2)$ \\
\hline $\mathrm{C}_{14}$ & $\mathrm{C}_{15}$ & $1.378(2)$ & $\mathrm{C}_{18}$ & $\mathrm{C}_{19}$ & $1.524(2)$ \\
\hline $\mathrm{C}_{14}$ & $\mathrm{C}_{13}$ & $1.3833(18)$ & $\mathrm{C}_{7}$ & $\mathrm{C}_{6}$ & $1.386(2)$ \\
\hline $\mathrm{C}_{2}$ & $\mathrm{C}_{3}$ & $1.4253(19)$ & $\mathrm{C}_{24}$ & $\mathrm{C}_{23}$ & $1.385(3)$ \\
\hline $\mathrm{C}_{2}$ & $\mathrm{C}_{1}$ & $1.3569(19)$ & $\mathrm{C}_{21}$ & $\mathrm{C}_{22}$ & $1.385(2)$ \\
\hline $\mathrm{C}_{12}$ & $\mathrm{C}_{13}$ & $1.3859(19)$ & $\mathrm{C}_{22}$ & $\mathrm{C}_{23}$ & $1.390(3)$ \\
\hline
\end{tabular}

Table S5. Bond Angles for $4 i$.

\begin{tabular}{|l|l|l|l|}
\hline Atom & Atom & Atom & \multicolumn{1}{|c|}{ Angle/ } \\
\hline $\mathrm{C}_{1}$ & $\mathrm{O}_{1}$ & $\mathrm{~N}_{1}$ & $107.50(9)$ \\
\hline $\mathrm{C}_{1}$ & $\mathrm{O}_{2}$ & $\mathrm{C}_{27}$ & $117.36(11)$ \\
\hline $\mathrm{C}_{3}$ & $\mathrm{~N}_{1}$ & $\mathrm{O}_{1}$ & $104.84(10)$ \\
\hline $\mathrm{O}_{4}$ & $\mathrm{~N}_{2}$ & $\mathrm{C}_{14}$ & $117.73(12)$ \\
\hline $\mathrm{O}_{5}$ & $\mathrm{~N}_{2}$ & $\mathrm{O}_{4}$ & $123.30(12)$ \\
\hline $\mathrm{O}_{5}$ & $\mathrm{~N}_{2}$ & $\mathrm{C}_{14}$ & $118.97(11)$ \\
\hline $\mathrm{C}_{12}$ & $\mathrm{C}_{11}$ & $\mathrm{C}_{10}$ & $120.31(11)$ \\
\hline $\mathrm{C}_{12}$ & $\mathrm{C}_{11}$ & $\mathrm{C}_{16}$ & $118.82(12)$ \\
\hline $\mathrm{C}_{16}$ & $\mathrm{C}_{11}$ & $\mathrm{C}_{10}$ & $120.84(12)$ \\
\hline $\mathrm{C}_{11}$ & $\mathrm{C}_{10}$ & $\mathrm{C}_{17}$ & $110.91(10)$ \\
\hline $\mathrm{C}_{2}$ & $\mathrm{C}_{10}$ & $\mathrm{C}_{11}$ & $112.51(11)$ \\
\hline $\mathrm{C}_{2}$ & $\mathrm{C}_{10}$ & $\mathrm{C}_{17}$ & $110.93(11)$ \\
\hline $\mathrm{C}_{15}$ & $\mathrm{C}_{14}$ & $\mathrm{~N}_{2}$ & $119.64(11)$ \\
\hline $\mathrm{C}_{15}$ & $\mathrm{C}_{14}$ & $\mathrm{C}_{13}$ & $122.10(12)$ \\
\hline $\mathrm{C}_{13}$ & $\mathrm{C}_{14}$ & $\mathrm{~N}_{2}$ & $118.26(13)$ \\
\hline $\mathrm{C}_{3}$ & $\mathrm{C}_{2}$ & $\mathrm{C}_{10}$ & $129.24(12)$ \\
\hline $\mathrm{C}_{1}$ & $\mathrm{C}_{2}$ & $\mathrm{C}_{10}$ & $128.03(12)$ \\
\hline $\mathrm{C}_{1}$ & $\mathrm{C}_{2}$ & $\mathrm{C}_{3}$ & $102.43(11)$ \\
\hline $\mathrm{C}_{13}$ & $\mathrm{C}_{12}$ & $\mathrm{C}_{11}$ & $121.21(12)$ \\
\hline $\mathrm{C}_{15}$ & $\mathrm{C}_{16}$ & $\mathrm{C}_{11}$ & $120.63(13)$ \\
\hline $\mathrm{C}_{14}$ & $\mathrm{C}_{15}$ & $\mathrm{C}_{16}$ & $118.81(12)$ \\
\hline $\mathrm{N}_{1}$ & $\mathrm{C}_{3}$ & $\mathrm{C}_{2}$ & $112.88(12)$ \\
\hline $\mathrm{N}_{1}$ & $\mathrm{C}_{3}$ & $\mathrm{C}_{4}$ & $117.72(11)$ \\
\hline $\mathrm{C}_{2}$ & $\mathrm{C}_{3}$ & $\mathrm{C}_{4}$ & $129.40(12)$ \\
\hline $\mathrm{O}_{1}$ & $\mathrm{C}_{1}$ & $\mathrm{C}_{2}$ & $112.35(11)$ \\
\hline $\mathrm{O}_{2}$ & $\mathrm{C}_{1}$ & $\mathrm{O}_{1}$ & $117.96(11)$ \\
\hline $\mathrm{O}_{2}$ & $\mathrm{C}_{1}$ & $\mathrm{C}_{2}$ & $129.69(12)$ \\
\hline & & & \\
\hline
\end{tabular}

\begin{tabular}{|l|l|l|c|}
\hline Atom & Atom & Atom & Angle $^{\circ}$ \\
\hline $\mathrm{C}_{9}$ & $\mathrm{C}_{4}$ & $\mathrm{C}_{3}$ & $120.88(11)$ \\
\hline $\mathrm{C}_{9}$ & $\mathrm{C}_{4}$ & $\mathrm{C}_{5}$ & $118.93(12)$ \\
\hline $\mathrm{C}_{5}$ & $\mathrm{C}_{4}$ & $\mathrm{C}_{3}$ & $120.15(12)$ \\
\hline $\mathrm{C}_{8}$ & $\mathrm{C}_{9}$ & $\mathrm{C}_{4}$ & $120.41(12)$ \\
\hline $\mathrm{O}_{3}$ & $\mathrm{C}_{26}$ & $\mathrm{C}_{17}$ & $122.74(14)$ \\
\hline $\mathrm{O}_{3}$ & $\mathrm{C}_{26}$ & $\mathrm{C}_{25}$ & $121.72(12)$ \\
\hline $\mathrm{C}_{25}$ & $\mathrm{C}_{26}$ & $\mathrm{C}_{17}$ & $115.47(11)$ \\
\hline $\mathrm{C}_{26}$ & $\mathrm{C}_{17}$ & $\mathrm{C}_{10}$ & $112.84(11)$ \\
\hline $\mathrm{C}_{26}$ & $\mathrm{C}_{17}$ & $\mathrm{C}_{18}$ & $106.13(11)$ \\
\hline $\mathrm{C}_{18}$ & $\mathrm{C}_{17}$ & $\mathrm{C}_{10}$ & $114.71(11)$ \\
\hline $\mathrm{C}_{20}$ & $\mathrm{C}_{25}$ & $\mathrm{C}_{26}$ & $121.33(12)$ \\
\hline $\mathrm{C}_{24}$ & $\mathrm{C}_{25}$ & $\mathrm{C}_{26}$ & $118.87(14)$ \\
\hline $\mathrm{C}_{24}$ & $\mathrm{C}_{25}$ & $\mathrm{C}_{20}$ & $119.77(15)$ \\
\hline $\mathrm{C}_{6}$ & $\mathrm{C}_{5}$ & $\mathrm{C}_{4}$ & $120.31(13)$ \\
\hline $\mathrm{C}_{14}$ & $\mathrm{C}_{13}$ & $\mathrm{C}_{12}$ & $118.38(13)$ \\
\hline $\mathrm{C}_{9}$ & $\mathrm{C}_{8}$ & $\mathrm{C}_{7}$ & $120.37(13)$ \\
\hline $\mathrm{C}_{25}$ & $\mathrm{C}_{20}$ & $\mathrm{C}_{19}$ & $120.47(13)$ \\
\hline $\mathrm{C}_{21}$ & $\mathrm{C}_{20}$ & $\mathrm{C}_{25}$ & $118.98(13)$ \\
\hline $\mathrm{C}_{21}$ & $\mathrm{C}_{20}$ & $\mathrm{C}_{19}$ & $120.53(13)$ \\
\hline $\mathrm{C}_{19}$ & $\mathrm{C}_{18}$ & $\mathrm{C}_{17}$ & $109.95(11)$ \\
\hline $\mathrm{C}_{6}$ & $\mathrm{C}_{7}$ & $\mathrm{C}_{8}$ & $119.49(13)$ \\
\hline $\mathrm{C}_{5}$ & $\mathrm{C}_{6}$ & $\mathrm{C}_{7}$ & $120.49(12)$ \\
\hline $\mathrm{C}_{20}$ & $\mathrm{C}_{19}$ & $\mathrm{C}_{18}$ & $112.24(12)$ \\
\hline $\mathrm{C}_{23}$ & $\mathrm{C}_{24}$ & $\mathrm{C}_{25}$ & $120.42(16)$ \\
\hline $\mathrm{C}_{22}$ & $\mathrm{C}_{21}$ & $\mathrm{C}_{20}$ & $120.84(16)$ \\
\hline $\mathrm{C}_{21}$ & $\mathrm{C}_{22}$ & $\mathrm{C}_{23}$ & $120.15(16)$ \\
\hline $\mathrm{C}_{24}$ & $\mathrm{C}_{23}$ & $\mathrm{C}_{22}$ & $119.80(14)$ \\
\hline & & & \\
\hline
\end{tabular}


Table S6. Hydrogen Atom Coordinates $\left(\AA^{\times} \times 10^{4}\right)$ and Isotropic Displacement Parameters $\left(\AA^{2} \times 10^{3}\right)$ for 4i.

\begin{tabular}{|l|l|l|l|l|}
\hline \multicolumn{1}{|c|}{ Atom } & \multicolumn{1}{c|}{$\boldsymbol{x}$} & \multicolumn{1}{c|}{$\boldsymbol{z}$} & U(eq) \\
\hline $\mathrm{H}_{10}$ & 5543 & 2487 & 5463 & 30 \\
\hline $\mathrm{H}_{12}$ & 3475 & 2669 & 4555 & 34 \\
\hline $\mathrm{H}_{16}$ & 6724 & 2157 & 3420 & 34 \\
\hline $\mathrm{H}_{15}$ & 5347 & 2134 & 2068 & 34 \\
\hline $\mathrm{H}_{9}$ & 4132 & 992 & 5264 & 34 \\
\hline $\mathrm{H}_{17}$ & 7733 & 3218 & 4735 & 31 \\
\hline $\mathrm{H}_{5}$ & 6779 & 194 & 7359 & 36 \\
\hline $\mathrm{H}_{13}$ & 2093 & 2650 & 3205 & 35 \\
\hline $\mathrm{H}_{8}$ & 2355 & 634 & 5984 & 37 \\
\hline $\mathrm{H}_{18 \mathrm{~A}}$ & 5710 & 4118 & 4280 & 36 \\
\hline $\mathrm{H}_{18 B}$ & 5728 & 4368 & 5263 & 36 \\
\hline $\mathrm{H}_{7}$ & 2779 & 82 & 7399 & 39 \\
\hline $\mathrm{H}_{6}$ & 4995 & -151 & 8075 & 38 \\
\hline $\mathrm{H}_{19 \mathrm{~A}}$ & 7764 & 4844 & 4273 & 39 \\
\hline $\mathrm{H}_{19 B}$ & 6828 & 5542 & 4660 & 39 \\
\hline $\mathrm{H}_{24}$ & 9648 & 3954 & 7347 & 46 \\
\hline $\mathrm{H}_{27 \mathrm{~A}}$ & 10590 & 1868 & 3964 & 57 \\
\hline $\mathrm{H}_{27 \mathrm{~B}}$ & 10139 & 880 & 4194 & 57 \\
\hline $\mathrm{H}_{27 \mathrm{C}}$ & 10807 & 1540 & 4936 & 57 \\
\hline $\mathrm{H}_{21}$ & 8996 & 6330 & 5347 & 46 \\
\hline $\mathrm{H}_{22}$ & 10521 & 6494 & 6621 & 52 \\
\hline $\mathrm{H}_{23}$ & 10826 & 5315 & 7635 & 53 \\
\hline
\end{tabular}

Crystal Data for $\mathrm{C}_{27} \mathrm{H}_{22} \mathrm{~N}_{2} \mathrm{O}_{5}(M=454.46 \mathrm{~g} / \mathrm{mol})$ : monoclinic, space group $\mathrm{P} 2{ }_{1} / \mathrm{c}$ (no. 14), $a=9.9953(2) \AA$, $b=14.5820(2) \AA, c=15.5765(2) \AA, \beta=99.4140(10)^{\circ}, V=2239.72(6) \AA^{3}, Z=4, T=100.00(10) \mathrm{K}$, $\mu(\mathrm{CuK \alpha})=0.769 \mathrm{~mm}^{-1}$, Dcalc $=1.348 \mathrm{~g} / \mathrm{cm}^{3}, 13848$ reflections measured $\left(8.36^{\circ} \leq 2 \Theta \leq 140.924^{\circ}\right), 4274$ unique $\left(R_{\text {int }}=0.0326, \mathrm{R}_{\text {sigma }}=0.0335\right)$ which were used in all calculations. The final $R_{1}$ was 0.0384 (I > $2 \sigma(\mathrm{I}))$ and $w R_{2}$ was 0.1042 (all data).

Details:

\section{Fixed Uiso}

At 1.2 times of: all $\mathrm{C}(\mathrm{H})$ groups, all $\mathrm{C}(\mathrm{H}, \mathrm{H})$ groups

At 1.5 times of: all $\mathrm{C}(\mathrm{H}, \mathrm{H}, \mathrm{H})$ groups

2a. Ternary $\mathrm{CH}$ refined with riding coordinates: $\mathrm{C}_{10}\left(\mathrm{H}_{10}\right), \mathrm{C}_{17}\left(\mathrm{H}_{17}\right)$

2b. Secondary $\mathrm{CH}_{2}$ refined with riding coordinates: $\mathrm{C}_{18}\left(\mathrm{H}_{18 \mathrm{~A}}, \mathrm{H}_{18 \mathrm{~B}}\right), \mathrm{C}_{19}\left(\mathrm{H}_{19 \mathrm{~A}}, \mathrm{H}_{19 \mathrm{~B}}\right)$

2c. Aromatic/amide $\mathrm{H}$ refined with riding coordinates: $\mathrm{C}_{12}\left(\mathrm{H}_{12}\right), \mathrm{C}_{16}\left(\mathrm{H}_{16}\right), \mathrm{C}_{15}\left(\mathrm{H}_{15}\right), \mathrm{C}_{9}\left(\mathrm{H}_{9}\right), \mathrm{C}_{5}\left(\mathrm{H}_{5}\right)$,

$\mathrm{C}_{13}\left(\mathrm{H}_{13}\right), \mathrm{C}_{8}\left(\mathrm{H}_{8}\right), \mathrm{C}_{7}\left(\mathrm{H}_{7}\right), \mathrm{C}_{6}\left(\mathrm{H}_{6}\right), \mathrm{C}_{24}\left(\mathrm{H}_{24}\right), \mathrm{C}_{21}\left(\mathrm{H}_{21}\right), \mathrm{C}_{22}\left(\mathrm{H}_{22}\right), \mathrm{C}_{23}\left(\mathrm{H}_{23}\right)$

2d. Idealised Me refined as rotating group: $\mathrm{C}_{27}\left(\mathrm{H}_{27 \mathrm{~A}}, \mathrm{H}_{27 \mathrm{~B}}, \mathrm{H}_{27 \mathrm{C}}\right)$ 
Single crystals of $\mathbf{7 b}$ were obtained by slow evaporation of dichloromethane solution at room temperature. (CCDC 2053526).

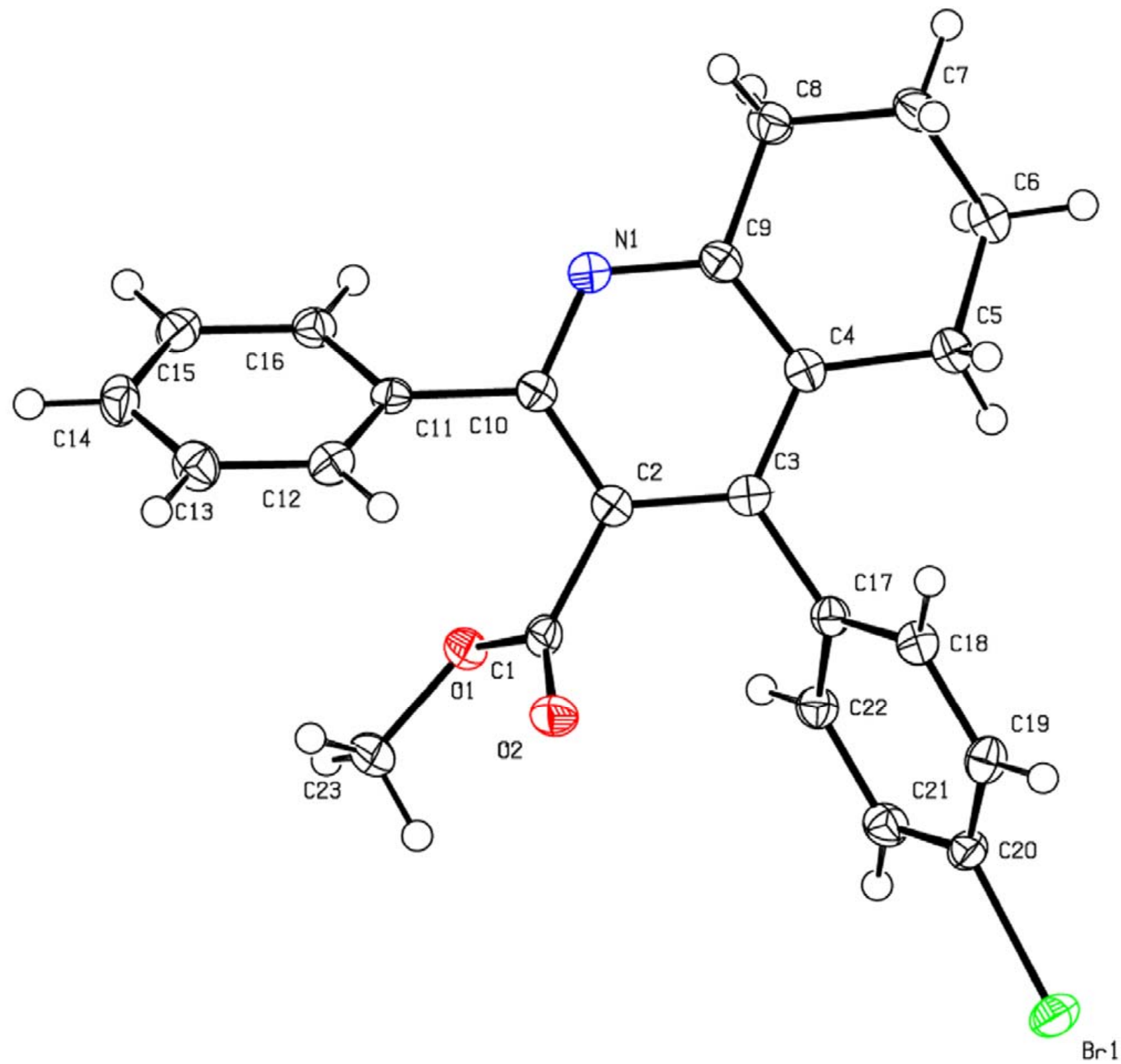

Molecular structure of compound $\mathbf{7 b}$, displacement parameters are drawn at 50\% probability level. 
Table S7. Crystal data and structure refinement for $\mathbf{7 b}$.

\begin{tabular}{|c|c|}
\hline Identification code & 7b (17504_CAT-12.65b) \\
\hline Empirical formula & $\mathrm{C}_{23} \mathrm{H}_{20} \mathrm{BrNO}_{2}$ \\
\hline Formula weight & 422.31 \\
\hline Temperature/K & $99.97(13)$ \\
\hline Crystal system & monoclinic \\
\hline Space group & $\mathrm{P} 2{ }_{1} / \mathrm{c}$ \\
\hline $\mathrm{a} / \AA$ & $11.5469(6)$ \\
\hline $\mathrm{b} / \AA$ & $14.4494(5)$ \\
\hline $\mathrm{c} / \AA ̊$ & $11.9365(5)$ \\
\hline$\alpha /^{\circ}$ & 90 \\
\hline$\beta / \circ$ & $108.360(5)$ \\
\hline$\gamma /{ }^{\circ}$ & 90 \\
\hline Volume $/ \AA^{3}$ & $1890.18(15)$ \\
\hline $\mathrm{Z}$ & 4 \\
\hline$\rho_{\text {calc }} g / \mathrm{cm}^{3}$ & 1.484 \\
\hline$\mu / \mathrm{mm}^{-1}$ & 3.103 \\
\hline $\mathrm{F}(000)$ & 864.0 \\
\hline Crystal size $/ \mathrm{mm}^{3}$ & $0.20 \times 0.18 \times 0.13$ \\
\hline Radiation & $\mathrm{CuK} \alpha(\lambda=1.54184)$ \\
\hline $2 \Theta$ range for data collection ${ }^{\circ}$ & 8.068 to 154.676 \\
\hline Index ranges & $-14 \leq \mathrm{h} \leq 14,-17 \leq \mathrm{k} \leq 18,-15 \leq 1 \leq 14$ \\
\hline Reflections collected & 15080 \\
\hline Independent reflections & $3914\left[\mathrm{R}_{\text {int }}=0.0437, \mathrm{R}_{\text {sigma }}=0.0387\right]$ \\
\hline Data/restraints/parameters & $3914 / 0 / 245$ \\
\hline Goodness-of-fit on $\mathrm{F}^{2}$ & 1.075 \\
\hline Final R indexes $[\mathrm{I}>=2 \sigma(\mathrm{I})]$ & $\mathrm{R}_{1}=0.0336, \mathrm{wR}_{2}=0.0903$ \\
\hline Final $\mathrm{R}$ indexes [all data] & $\mathrm{R}_{1}=0.0372, \mathrm{wR}_{2}=0.0928$ \\
\hline Largest diff. peak/hole / e $\AA^{-3}$ & $0.45 /-0.45$ \\
\hline
\end{tabular}

Table S8. Fractional Atomic Coordinates $\left(\times 10^{4}\right)$ and Equivalent Isotropic Displacement Parameters $\left(\AA^{2} \times 10^{3}\right)$ for $7 \mathrm{~b} . \mathrm{U}_{\mathrm{eq}}$ is defined as $1 / 3$ of of the trace of the orthogonalised $U_{\mathrm{IJ}}$ tensor.

\begin{tabular}{|c|c|c|c|c|}
\hline Atom & $x$ & $y$ & $z$ & $\mathrm{U}(\mathbf{e q})$ \\
\hline $\mathrm{Br}_{1}$ & $1981.2(2)$ & $1385.6(2)$ & $5368.2(2)$ & $25.34(10)$ \\
\hline $\mathrm{O}_{2}$ & $1813.7(13)$ & $5535.3(10)$ & $5108.5(12)$ & $23.3(3)$ \\
\hline $\mathrm{O}_{1}$ & $596.7(12)$ & $6062.2(9)$ & $3355.0(12)$ & $20.8(3)$ \\
\hline $\mathrm{N}_{1}$ & $3771.1(14)$ & $6771.0(11)$ & $2594.7(13)$ & $17.6(3)$ \\
\hline $\mathrm{C}_{10}$ & 2935.4(16) & $6676.4(13)$ & $3145.5(15)$ & $16.9(3)$ \\
\hline $\mathrm{C}_{9}$ & $4383.6(16)$ & $6016.7(13)$ & $2435.1(16)$ & $18.2(4)$ \\
\hline $\mathrm{C}_{11}$ & $2345.5(16)$ & $7547.9(12)$ & $3354.9(16)$ & $17.3(3)$ \\
\hline $\mathrm{C}_{3}$ & $3302.4(16)$ & $5030.7(12)$ & $3394.5(15)$ & $16.8(3)$ \\
\hline $\mathrm{C}_{16}$ & 1811.9(16) & $8140.5(13)$ & $2410.5(16)$ & $19.3(4)$ \\
\hline $\mathrm{C}_{17}$ & $3020.8(16)$ & $4116.3(12)$ & $3822.4(15)$ & $16.8(3)$ \\
\hline $\mathrm{C}_{4}$ & $4201.6(16)$ & $5130.9(12)$ & 2841.4(15) & $17.3(3)$ \\
\hline $\mathrm{C}_{1}$ & 1663.3(16) & $5771.0(12)$ & $4103.7(16)$ & $18.0(3)$ \\
\hline $\mathrm{C}_{2}$ & $2645.7(16)$ & $5816.5(12)$ & $3529.6(15)$ & $16.8(3)$ \\
\hline $\mathrm{C}_{21}$ & 1541.9(17) & 2923.4(13) & $3810.6(17)$ & $21.3(4)$ \\
\hline $\mathrm{C}_{20}$ & $2424.0(17)$ & 2477.1(12) & $4708.4(16)$ & $18.9(3)$ \\
\hline $\mathrm{C}_{15}$ & $1260.4(17)$ & $8958.3(13)$ & $2590.0(17)$ & $21.6(4)$ \\
\hline $\mathrm{C}_{5}$ & 4941.9(17) & $4315.3(13)$ & $2646.9(16)$ & $20.6(4)$ \\
\hline $\mathrm{C}_{18}$ & $3897.8(18)$ & $3627.8(12)$ & $4698.4(17)$ & $19.6(4)$ \\
\hline
\end{tabular}




\begin{tabular}{|l|l|l|l|l|}
\hline $\mathrm{C}_{19}$ & $3604.0(17)$ & $2809.7(13)$ & $5153.1(16)$ & $20.5(4)$ \\
\hline $\mathrm{C}_{22}$ & $1850.0(17)$ & $3746.1(13)$ & $3375.0(16)$ & $19.0(4)$ \\
\hline $\mathrm{C}_{12}$ & $2350.5(17)$ & $7797.9(13)$ & $4485.2(16)$ & $20.5(4)$ \\
\hline $\mathrm{C}_{13}$ & $1799.0(19)$ & $8612.9(13)$ & $4660.9(19)$ & $24.0(4)$ \\
\hline $\mathrm{C}_{8}$ & $5281.5(18)$ & $6195.8(14)$ & $1776.6(17)$ & $22.5(4)$ \\
\hline $\mathrm{C}_{7}$ & $6160.3(17)$ & $5396.5(14)$ & $1840.6(18)$ & $23.1(4)$ \\
\hline $\mathrm{C}_{6}$ & $5452.7(18)$ & $4486.4(14)$ & $1633.6(17)$ & $23.1(4)$ \\
\hline $\mathrm{C}_{14}$ & $1241.2(18)$ & $9188.0(13)$ & $3713.2(18)$ & $23.7(4)$ \\
\hline $\mathrm{C}_{23}$ & $-353.1(19)$ & $6256.3(15)$ & $3876(2)$ & $27.0(4)$ \\
\hline
\end{tabular}

Table S9. Anisotropic Displacement Parameters $\left(\AA^{2} \times 10^{3}\right)$ for $7 \mathrm{~b}$. The Anisotropic displacement factor exponent takes the form: $-2 \pi^{2}\left[h^{2} a *{ }^{2} U_{11}+2 h k a * b * U_{12}+\ldots\right]$.

\begin{tabular}{|l|l|l|l|l|l|l|}
\hline \multicolumn{1}{|c|}{$\mathbf{A t o m}$} & \multicolumn{1}{|c|}{$\mathbf{U}_{\mathbf{1 1}}$} & \multicolumn{1}{|c|}{$\mathbf{U}_{\mathbf{2 2}}$} & \multicolumn{1}{c|}{$\mathbf{U}_{\mathbf{3 3}}$} & \multicolumn{1}{|c|}{$\mathbf{U}_{\mathbf{2 3}}$} & \multicolumn{1}{|c|}{$\mathbf{U}_{\mathbf{1 3}}$} & \multicolumn{1}{|c|}{$\mathbf{U}_{\mathbf{1 2}}$} \\
\hline $\mathrm{Br}_{1}$ & $28.05(14)$ & $19.47(14)$ & $29.11(14)$ & $3.64(7)$ & $9.90(10)$ & $-3.50(7)$ \\
\hline $\mathrm{O}_{2}$ & $26.3(7)$ & $25.3(7)$ & $21.2(6)$ & $3.5(5)$ & $11.8(5)$ & $1.2(5)$ \\
\hline $\mathrm{O}_{1}$ & $16.8(6)$ & $21.2(6)$ & $25.1(7)$ & $0.2(5)$ & $7.8(5)$ & $-0.1(5)$ \\
\hline $\mathrm{N}_{1}$ & $17.2(7)$ & $18.6(7)$ & $16.5(7)$ & $0.2(5)$ & $4.5(6)$ & $-0.9(6)$ \\
\hline $\mathrm{C}_{10}$ & $15.4(8)$ & $19.3(8)$ & $14.9(8)$ & $0.7(6)$ & $3.5(6)$ & $0.7(7)$ \\
\hline $\mathrm{C}_{9}$ & $15.0(8)$ & $21.5(9)$ & $17.3(8)$ & $-0.2(7)$ & $4.2(6)$ & $0.3(7)$ \\
\hline $\mathrm{C}_{11}$ & $14.3(8)$ & $16.5(8)$ & $21.6(8)$ & $0.7(7)$ & $6.6(6)$ & $-2.3(6)$ \\
\hline $\mathrm{C}_{3}$ & $16.3(8)$ & $19.1(8)$ & $13.2(8)$ & $-0.9(6)$ & $2.2(6)$ & $-1.8(6)$ \\
\hline $\mathrm{C}_{16}$ & $17.1(8)$ & $20.5(9)$ & $20.4(8)$ & $0.6(7)$ & $5.9(7)$ & $-1.4(7)$ \\
\hline $\mathrm{C}_{17}$ & $18.6(8)$ & $16.4(8)$ & $16.0(8)$ & $-2.5(6)$ & $6.3(6)$ & $-0.9(6)$ \\
\hline $\mathrm{C}_{4}$ & $15.1(8)$ & $20.0(8)$ & $15.0(8)$ & $-1.6(6)$ & $2.1(6)$ & $0.7(6)$ \\
\hline $\mathrm{C}_{1}$ & $19.0(8)$ & $13.8(8)$ & $21.7(9)$ & $-2.7(6)$ & $7.2(7)$ & $-0.9(6)$ \\
\hline $\mathrm{C}_{2}$ & $15.3(8)$ & $19.8(8)$ & $14.2(8)$ & $-0.5(6)$ & $3.0(6)$ & $-0.1(6)$ \\
\hline $\mathrm{C}_{21}$ & $17.6(8)$ & $22.6(9)$ & $24.1(9)$ & $-3.3(7)$ & $7.1(7)$ & $-2.8(7)$ \\
\hline $\mathrm{C}_{20}$ & $24.5(9)$ & $14.6(8)$ & $19.5(8)$ & $-0.3(6)$ & $9.8(7)$ & $-0.9(7)$ \\
\hline $\mathrm{C}_{15}$ & $18.4(9)$ & $19.1(9)$ & $25.6(9)$ & $3.0(7)$ & $4.3(7)$ & $-0.6(7)$ \\
\hline $\mathrm{C}_{5}$ & $18.5(8)$ & $22.2(9)$ & $21.1(9)$ & $-0.6(7)$ & $6.1(7)$ & $2.7(7)$ \\
\hline $\mathrm{C}_{18}$ & $18.3(9)$ & $20.1(9)$ & $19.6(9)$ & $-1.7(6)$ & $4.8(7)$ & $-0.4(6)$ \\
\hline $\mathrm{C}_{19}$ & $21.7(9)$ & $19.4(8)$ & $18.2(8)$ & $0.7(7)$ & $3.3(7)$ & $1.9(7)$ \\
\hline $\mathrm{C}_{22}$ & $17.0(8)$ & $20.2(8)$ & $19.0(8)$ & $0.9(7)$ & $4.5(7)$ & $1.0(7)$ \\
\hline $\mathrm{C}_{12}$ & $19.9(8)$ & $20.3(9)$ & $19.5(8)$ & $0.4(7)$ & $3.7(7)$ & $-1.9(7)$ \\
\hline $\mathrm{C}_{13}$ & $24.7(10)$ & $25.1(10)$ & $22.4(9)$ & $-5.3(7)$ & $7.6(8)$ & $-1.2(7)$ \\
\hline $\mathrm{C}_{8}$ & $22.9(9)$ & $24.7(9)$ & $22.3(9)$ & $3.2(7)$ & $10.5(7)$ & $2.4(8)$ \\
\hline $\mathrm{C}_{7}$ & $17.9(8)$ & $28.3(10)$ & $25.0(9)$ & $2.0(7)$ & $9.4(7)$ & $2.4(7)$ \\
\hline $\mathrm{C}_{6}$ & $20.5(9)$ & $25.0(9)$ & $24.4(9)$ & $-1.8(7)$ & $8.0(7)$ & $3.9(7)$ \\
\hline $\mathrm{C}_{14}$ & $20.1(9)$ & $17.4(8)$ & $32.3(10)$ & $-5.0(7)$ & $6.4(7)$ & $0.3(7)$ \\
\hline $\mathrm{C}_{23}$ & $20.0(9)$ & $25.8(10)$ & $39.6(12)$ & $0.5(8)$ & $15.8(8)$ & $0.3(8)$ \\
\hline
\end{tabular}


Table S10. Bond Lengths for $7 \mathrm{~b}$.

\begin{tabular}{|l|l|l|}
\hline Atom & Atom & Length/Å \\
\hline $\mathrm{Br}_{1}$ & $\mathrm{C}_{20}$ & $1.9034(18)$ \\
\hline $\mathrm{O}_{2}$ & $\mathrm{C}_{1}$ & $1.205(2)$ \\
\hline $\mathrm{O}_{1}$ & $\mathrm{C}_{1}$ & $1.341(2)$ \\
\hline $\mathrm{O}_{1}$ & $\mathrm{C}_{23}$ & $1.448(2)$ \\
\hline $\mathrm{N}_{1}$ & $\mathrm{C}_{10}$ & $1.335(2)$ \\
\hline $\mathrm{N}_{1}$ & $\mathrm{C}_{9}$ & $1.345(2)$ \\
\hline $\mathrm{C}_{10}$ & $\mathrm{C}_{11}$ & $1.490(3)$ \\
\hline $\mathrm{C}_{10}$ & $\mathrm{C}_{2}$ & $1.401(3)$ \\
\hline $\mathrm{C}_{9}$ & $\mathrm{C}_{4}$ & $1.408(3)$ \\
\hline $\mathrm{C}_{9}$ & $\mathrm{C}_{8}$ & $1.508(3)$ \\
\hline $\mathrm{C}_{11}$ & $\mathrm{C}_{16}$ & $1.395(3)$ \\
\hline $\mathrm{C}_{11}$ & $\mathrm{C}_{12}$ & $1.395(3)$ \\
\hline $\mathrm{C}_{3}$ & $\mathrm{C}_{17}$ & $1.489(2)$ \\
\hline $\mathrm{C}_{3}$ & $\mathrm{C}_{4}$ & $1.402(3)$ \\
\hline $\mathrm{C}_{3}$ & $\mathrm{C}_{2}$ & $1.403(3)$ \\
\hline
\end{tabular}

\begin{tabular}{|l|l|l|}
\hline Atom & Atom & Length/A \\
\hline $\mathrm{C}_{16}$ & $\mathrm{C}_{15}$ & $1.391(3)$ \\
\hline $\mathrm{C}_{17}$ & $\mathrm{C}_{18}$ & $1.396(3)$ \\
\hline $\mathrm{C}_{17}$ & $\mathrm{C}_{22}$ & $1.394(3)$ \\
\hline $\mathrm{C}_{4}$ & $\mathrm{C}_{5}$ & $1.516(3)$ \\
\hline $\mathrm{C}_{1}$ & $\mathrm{C}_{2}$ & $1.500(3)$ \\
\hline $\mathrm{C}_{21}$ & $\mathrm{C}_{20}$ & $1.384(3)$ \\
\hline $\mathrm{C}_{21}$ & $\mathrm{C}_{22}$ & $1.388(3)$ \\
\hline $\mathrm{C}_{20}$ & $\mathrm{C}_{19}$ & $1.383(3)$ \\
\hline $\mathrm{C}_{15}$ & $\mathrm{C}_{14}$ & $1.388(3)$ \\
\hline $\mathrm{C}_{5}$ & $\mathrm{C}_{6}$ & $1.524(3)$ \\
\hline $\mathrm{C}_{18}$ & $\mathrm{C}_{19}$ & $1.387(3)$ \\
\hline $\mathrm{C}_{12}$ & $\mathrm{C}_{13}$ & $1.386(3)$ \\
\hline $\mathrm{C}_{13}$ & $\mathrm{C}_{14}$ & $1.388(3)$ \\
\hline $\mathrm{C}_{8}$ & $\mathrm{C}_{7}$ & $1.523(3)$ \\
\hline $\mathrm{C}_{7}$ & $\mathrm{C}_{6}$ & $1.527(3)$ \\
\hline
\end{tabular}

Table S11. Bond Angles for $7 \mathrm{~b}$.

\begin{tabular}{|l|l|l|c|}
\hline Atom & Atom & Atom & Angle $^{\circ}$ \\
\hline $\mathrm{C}_{1}$ & $\mathrm{O}_{1}$ & $\mathrm{C}_{23}$ & $115.80(15)$ \\
\hline $\mathrm{C}_{10}$ & $\mathrm{~N}_{1}$ & $\mathrm{C}_{9}$ & $118.74(16)$ \\
\hline $\mathrm{N}_{1}$ & $\mathrm{C}_{10}$ & $\mathrm{C}_{11}$ & $115.84(16)$ \\
\hline $\mathrm{N}_{1}$ & $\mathrm{C}_{10}$ & $\mathrm{C}_{2}$ & $122.42(17)$ \\
\hline $\mathrm{C}_{2}$ & $\mathrm{C}_{10}$ & $\mathrm{C}_{11}$ & $121.72(16)$ \\
\hline $\mathrm{N}_{1}$ & $\mathrm{C}_{9}$ & $\mathrm{C}_{4}$ & $123.07(17)$ \\
\hline $\mathrm{N}_{1}$ & $\mathrm{C}_{9}$ & $\mathrm{C}_{8}$ & $114.42(16)$ \\
\hline $\mathrm{C}_{4}$ & $\mathrm{C}_{9}$ & $\mathrm{C}_{8}$ & $122.51(17)$ \\
\hline $\mathrm{C}_{16}$ & $\mathrm{C}_{11}$ & $\mathrm{C}_{10}$ & $119.48(16)$ \\
\hline $\mathrm{C}_{12}$ & $\mathrm{C}_{11}$ & $\mathrm{C}_{10}$ & $121.22(16)$ \\
\hline $\mathrm{C}_{12}$ & $\mathrm{C}_{11}$ & $\mathrm{C}_{16}$ & $119.27(17)$ \\
\hline $\mathrm{C}_{4}$ & $\mathrm{C}_{3}$ & $\mathrm{C}_{17}$ & $121.81(16)$ \\
\hline $\mathrm{C}_{2}$ & $\mathrm{C}_{3}$ & $\mathrm{C}_{17}$ & $119.64(16)$ \\
\hline $\mathrm{C}_{2}$ & $\mathrm{C}_{3}$ & $\mathrm{C}_{4}$ & $118.54(16)$ \\
\hline $\mathrm{C}_{15}$ & $\mathrm{C}_{16}$ & $\mathrm{C}_{11}$ & $120.28(18)$ \\
\hline $\mathrm{C}_{18}$ & $\mathrm{C}_{17}$ & $\mathrm{C}_{3}$ & $121.34(16)$ \\
\hline $\mathrm{C}_{22}$ & $\mathrm{C}_{17}$ & $\mathrm{C}_{3}$ & $119.95(16)$ \\
\hline $\mathrm{C}_{22}$ & $\mathrm{C}_{17}$ & $\mathrm{C}_{18}$ & $118.67(17)$ \\
\hline $\mathrm{C}_{9}$ & $\mathrm{C}_{4}$ & $\mathrm{C}_{5}$ & $120.09(16)$ \\
\hline $\mathrm{C}_{3}$ & $\mathrm{C}_{4}$ & $\mathrm{C}_{9}$ & $117.97(16)$ \\
\hline $\mathrm{C}_{3}$ & $\mathrm{C}_{4}$ & $\mathrm{C}_{5}$ & $121.92(16)$ \\
\hline
\end{tabular}

\begin{tabular}{|c|c|c|c|}
\hline Atom & Atom & Atom & Angle $/^{\circ}$ \\
\hline $\mathrm{O}_{2}$ & $\mathrm{C}_{1}$ & $\mathrm{O}_{1}$ & $124.46(17)$ \\
\hline $\mathrm{O}_{2}$ & $\mathrm{C}_{1}$ & $\mathrm{C}_{2}$ & $125.04(16)$ \\
\hline $\mathrm{O}_{1}$ & $\mathrm{C}_{1}$ & $\mathrm{C}_{2}$ & $110.46(15)$ \\
\hline $\mathrm{C}_{10}$ & $\mathrm{C}_{2}$ & $\mathrm{C}_{3}$ & $119.14(17)$ \\
\hline $\mathrm{C}_{10}$ & $\mathrm{C}_{2}$ & $\mathrm{C}_{1}$ & $118.77(16)$ \\
\hline $\mathrm{C}_{3}$ & $\mathrm{C}_{2}$ & $\mathrm{C}_{1}$ & $122.07(16)$ \\
\hline $\mathrm{C}_{20}$ & $\mathrm{C}_{21}$ & $\mathrm{C}_{22}$ & $118.44(17)$ \\
\hline $\mathrm{C}_{21}$ & $\mathrm{C}_{20}$ & $\mathrm{Br}_{1}$ & $118.59(14)$ \\
\hline $\mathrm{C}_{19}$ & $\mathrm{C}_{20}$ & $\mathrm{Br}_{1}$ & $119.31(14)$ \\
\hline $\mathrm{C}_{19}$ & $\mathrm{C}_{20}$ & $\mathrm{C}_{21}$ & $122.09(17)$ \\
\hline $\mathrm{C}_{14}$ & $\mathrm{C}_{15}$ & $\mathrm{C}_{16}$ & $119.96(18)$ \\
\hline $\mathrm{C}_{4}$ & $\mathrm{C}_{5}$ & $\mathrm{C}_{6}$ & $111.92(16)$ \\
\hline $\mathrm{C}_{19}$ & $\mathrm{C}_{18}$ & $\mathrm{C}_{17}$ & $120.99(18)$ \\
\hline $\mathrm{C}_{20}$ & $\mathrm{C}_{19}$ & $\mathrm{C}_{18}$ & $118.61(17)$ \\
\hline $\mathrm{C}_{21}$ & $\mathrm{C}_{22}$ & $\mathrm{C}_{17}$ & $121.14(17)$ \\
\hline $\mathrm{C}_{13}$ & $\mathrm{C}_{12}$ & $\mathrm{C}_{11}$ & $120.28(18)$ \\
\hline $\mathrm{C}_{12}$ & $\mathrm{C}_{13}$ & $\mathrm{C}_{14}$ & $120.19(19)$ \\
\hline $\mathrm{C}_{9}$ & $\mathrm{C}_{8}$ & $\mathrm{C}_{7}$ & $113.60(16)$ \\
\hline $\mathrm{C}_{8}$ & $\mathrm{C}_{7}$ & $\mathrm{C}_{6}$ & $109.32(16)$ \\
\hline $\mathrm{C}_{5}$ & $\mathrm{C}_{6}$ & $\mathrm{C}_{7}$ & $109.42(16)$ \\
\hline $\mathrm{C}_{13}$ & $\mathrm{C}_{14}$ & $\mathrm{C}_{15}$ & $119.97(18)$ \\
\hline
\end{tabular}


Table S12. Torsion Angles for $7 \mathbf{b}$.

\begin{tabular}{|l|l|l|l|l|}
\hline $\mathbf{A}$ & \multicolumn{1}{|c|}{$\mathbf{B}$} & \multicolumn{1}{|c|}{$\mathbf{C}$} & \multicolumn{1}{|c|}{$\mathbf{D}_{18}$} & \multicolumn{1}{|c|}{ Angle/ } \\
\hline $\mathrm{Br}_{1}$ & $\mathrm{C}_{20}$ & $\mathrm{C}_{19}$ & $\mathrm{C}_{18}$ & $-17.19(14)$ \\
\hline $\mathrm{O}_{2}$ & $\mathrm{C}_{1}$ & $\mathrm{C}_{2}$ & $\mathrm{C}_{10}$ & $-117.0(2)$ \\
\hline $\mathrm{O}_{2}$ & $\mathrm{C}_{1}$ & $\mathrm{C}_{2}$ & $\mathrm{C}_{3}$ & $61.3(3)$ \\
\hline $\mathrm{O}_{1}$ & $\mathrm{C}_{1}$ & $\mathrm{C}_{2}$ & $\mathrm{C}_{10}$ & $60.8(2)$ \\
\hline $\mathrm{O}_{1}$ & $\mathrm{C}_{1}$ & $\mathrm{C}_{2}$ & $\mathrm{C}_{3}$ & $-120.92(17)$ \\
\hline $\mathrm{N}_{1}$ & $\mathrm{C}_{10}$ & $\mathrm{C}_{11}$ & $\mathrm{C}_{16}$ & $53.0(2)$ \\
\hline $\mathrm{N}_{1}$ & $\mathrm{C}_{10}$ & $\mathrm{C}_{11}$ & $\mathrm{C}_{12}$ & $-124.91(18)$ \\
\hline $\mathrm{N}_{1}$ & $\mathrm{C}_{10}$ & $\mathrm{C}_{2}$ & $\mathrm{C}_{3}$ & $3.8(3)$ \\
\hline $\mathrm{N}_{1}$ & $\mathrm{C}_{10}$ & $\mathrm{C}_{2}$ & $\mathrm{C}_{1}$ & $-177.91(15)$ \\
\hline $\mathrm{N}_{1}$ & $\mathrm{C}_{9}$ & $\mathrm{C}_{4}$ & $\mathrm{C}_{3}$ & $2.7(3)$ \\
\hline $\mathrm{N}_{1}$ & $\mathrm{C}_{9}$ & $\mathrm{C}_{4}$ & $\mathrm{C}_{5}$ & $-178.96(16)$ \\
\hline $\mathrm{N}_{1}$ & $\mathrm{C}_{9}$ & $\mathrm{C}_{8}$ & $\mathrm{C}_{7}$ & $167.11(16)$ \\
\hline $\mathrm{C}_{10}$ & $\mathrm{~N}_{1}$ & $\mathrm{C}_{9}$ & $\mathrm{C}_{4}$ & $-1.2(3)$ \\
\hline $\mathrm{C}_{10}$ & $\mathrm{~N}_{1}$ & $\mathrm{C}_{9}$ & $\mathrm{C}_{8}$ & $178.49(15)$ \\
\hline $\mathrm{C}_{10}$ & $\mathrm{C}_{11}$ & $\mathrm{C}_{16}$ & $\mathrm{C}_{15}$ & $-179.80(17)$ \\
\hline $\mathrm{C}_{10}$ & $\mathrm{C}_{11}$ & $\mathrm{C}_{12}$ & $\mathrm{C}_{13}$ & $179.83(17)$ \\
\hline $\mathrm{C}_{9}$ & $\mathrm{~N}_{1}$ & $\mathrm{C}_{10}$ & $\mathrm{C}_{11}$ & $176.51(15)$ \\
\hline $\mathrm{C}_{9}$ & $\mathrm{~N}_{1}$ & $\mathrm{C}_{10}$ & $\mathrm{C}_{2}$ & $-2.1(3)$ \\
\hline $\mathrm{C}_{9}$ & $\mathrm{C}_{4}$ & $\mathrm{C}_{5}$ & $\mathrm{C}_{6}$ & $-21.8(2)$ \\
\hline $\mathrm{C}_{9}$ & $\mathrm{C}_{8}$ & $\mathrm{C}_{7}$ & $\mathrm{C}_{6}$ & $44.5(2)$ \\
\hline $\mathrm{C}_{11}$ & $\mathrm{C}_{10}$ & $\mathrm{C}_{2}$ & $\mathrm{C}_{3}$ & $-174.76(16)$ \\
\hline $\mathrm{C}_{11}$ & $\mathrm{C}_{10}$ & $\mathrm{C}_{2}$ & $\mathrm{C}_{1}$ & $3.6(2)$ \\
\hline $\mathrm{C}_{11}$ & $\mathrm{C}_{16}$ & $\mathrm{C}_{15}$ & $\mathrm{C}_{14}$ & $0.1(3)$ \\
\hline $\mathrm{C}_{11}$ & $\mathrm{C}_{12}$ & $\mathrm{C}_{13}$ & $\mathrm{C}_{14}$ & $-0.2(3)$ \\
\hline $\mathrm{C}_{3}$ & $\mathrm{C}_{17}$ & $\mathrm{C}_{18}$ & $\mathrm{C}_{19}$ & $175.26(17)$ \\
\hline $\mathrm{C}_{3}$ & $\mathrm{C}_{17}$ & $\mathrm{C}_{22}$ & $\mathrm{C}_{21}$ & $-175.94(17)$ \\
\hline $\mathrm{C}_{3}$ & $\mathrm{C}_{4}$ & $\mathrm{C}_{5}$ & $\mathrm{C}_{6}$ & $156.52(16)$ \\
\hline $\mathrm{C}_{16}$ & $\mathrm{C}_{11}$ & $\mathrm{C}_{12}$ & $\mathrm{C}_{13}$ & $1.9(3)$ \\
\hline $\mathrm{C}_{16}$ & $\mathrm{C}_{15}$ & $\mathrm{C}_{14}$ & $\mathrm{C}_{13}$ & $1.6(3)$ \\
\hline $\mathrm{C}_{17}$ & $\mathrm{C}_{3}$ & $\mathrm{C}_{4}$ & $\mathrm{C}_{9}$ & $178.25(15)$ \\
\hline & & & & \\
\hline
\end{tabular}

\begin{tabular}{|l|l|l|l|l|}
\hline \multicolumn{1}{|c|}{$\mathbf{A}$} & \multicolumn{1}{|c|}{$\mathbf{B}$} & \multicolumn{1}{|c|}{$\mathbf{C}$} & \multicolumn{1}{|c|}{ A } & \multicolumn{1}{|c|}{ Angle ${ }^{\circ}$} \\
\hline $\mathrm{C}_{17}$ & $\mathrm{C}_{3}$ & $\mathrm{C}_{4}$ & $\mathrm{C}_{5}$ & $-0.1(2)$ \\
\hline $\mathrm{C}_{17}$ & $\mathrm{C}_{3}$ & $\mathrm{C}_{2}$ & $\mathrm{C}_{10}$ & $178.71(15)$ \\
\hline $\mathrm{C}_{17}$ & $\mathrm{C}_{3}$ & $\mathrm{C}_{2}$ & $\mathrm{C}_{1}$ & $0.4(2)$ \\
\hline $\mathrm{C}_{17}$ & $\mathrm{C}_{18}$ & $\mathrm{C}_{19}$ & $\mathrm{C}_{20}$ & $0.9(3)$ \\
\hline $\mathrm{C}_{4}$ & $\mathrm{C}_{9}$ & $\mathrm{C}_{8}$ & $\mathrm{C}_{7}$ & $-13.2(2)$ \\
\hline $\mathrm{C}_{4}$ & $\mathrm{C}_{3}$ & $\mathrm{C}_{17}$ & $\mathrm{C}_{18}$ & $62.1(2)$ \\
\hline $\mathrm{C}_{4}$ & $\mathrm{C}_{3}$ & $\mathrm{C}_{17}$ & $\mathrm{C}_{22}$ & $-120.02(19)$ \\
\hline $\mathrm{C}_{4}$ & $\mathrm{C}_{3}$ & $\mathrm{C}_{2}$ & $\mathrm{C}_{10}$ & $-2.1(2)$ \\
\hline $\mathrm{C}_{4}$ & $\mathrm{C}_{3}$ & $\mathrm{C}_{2}$ & $\mathrm{C}_{1}$ & $179.61(15)$ \\
\hline $\mathrm{C}_{4}$ & $\mathrm{C}_{5}$ & $\mathrm{C}_{6}$ & $\mathrm{C}_{7}$ & $53.6(2)$ \\
\hline $\mathrm{C}_{2}$ & $\mathrm{C}_{10}$ & $\mathrm{C}_{11}$ & $\mathrm{C}_{16}$ & $-128.40(18)$ \\
\hline $\mathrm{C}_{2}$ & $\mathrm{C}_{10}$ & $\mathrm{C}_{11}$ & $\mathrm{C}_{12}$ & $53.7(2)$ \\
\hline $\mathrm{C}_{2}$ & $\mathrm{C}_{3}$ & $\mathrm{C}_{17}$ & $\mathrm{C}_{18}$ & $-118.72(19)$ \\
\hline $\mathrm{C}_{2}$ & $\mathrm{C}_{3}$ & $\mathrm{C}_{17}$ & $\mathrm{C}_{22}$ & $59.1(2)$ \\
\hline $\mathrm{C}_{2}$ & $\mathrm{C}_{3}$ & $\mathrm{C}_{4}$ & $\mathrm{C}_{9}$ & $-0.9(2)$ \\
\hline $\mathrm{C}_{2}$ & $\mathrm{C}_{3}$ & $\mathrm{C}_{4}$ & $\mathrm{C}_{5}$ & $-179.24(16)$ \\
\hline $\mathrm{C}_{21}$ & $\mathrm{C}_{20}$ & $\mathrm{C}_{19}$ & $\mathrm{C}_{18}$ & $1.5(3)$ \\
\hline $\mathrm{C}_{20}$ & $\mathrm{C}_{21}$ & $\mathrm{C}_{22}$ & $\mathrm{C}_{17}$ & $0.3(3)$ \\
\hline $\mathrm{C}_{18}$ & $\mathrm{C}_{17}$ & $\mathrm{C}_{22}$ & $\mathrm{C}_{21}$ & $2.0(3)$ \\
\hline $\mathrm{C}_{22}$ & $\mathrm{C}_{17}$ & $\mathrm{C}_{18}$ & $\mathrm{C}_{19}$ & $-2.6(3)$ \\
\hline $\mathrm{C}_{22}$ & $\mathrm{C}_{21}$ & $\mathrm{C}_{20}$ & $\mathrm{Br}_{1}$ & $176.57(14)$ \\
\hline $\mathrm{C}_{22}$ & $\mathrm{C}_{21}$ & $\mathrm{C}_{20}$ & $\mathrm{C}_{19}$ & $-2.1(3)$ \\
\hline $\mathrm{C}_{12}$ & $\mathrm{C}_{11}$ & $\mathrm{C}_{16}$ & $\mathrm{C}_{15}$ & $-1.8(3)$ \\
\hline $\mathrm{C}_{12}$ & $\mathrm{C}_{13}$ & $\mathrm{C}_{14}$ & $\mathrm{C}_{15}$ & $-1.5(3)$ \\
\hline $\mathrm{C}_{8}$ & $\mathrm{C}_{9}$ & $\mathrm{C}_{4}$ & $\mathrm{C}_{3}$ & $-176.98(16)$ \\
\hline $\mathrm{C}_{8}$ & $\mathrm{C}_{9}$ & $\mathrm{C}_{4}$ & $\mathrm{C}_{5}$ & $1.4(3)$ \\
\hline $\mathrm{C}_{8}$ & $\mathrm{C}_{7}$ & $\mathrm{C}_{6}$ & $\mathrm{C}_{5}$ & $-65.7(2)$ \\
\hline $\mathrm{C}_{23}$ & $\mathrm{O}_{1}$ & $\mathrm{C}_{1}$ & $\mathrm{O}_{2}$ & $11.5(3)$ \\
\hline $\mathrm{C}_{23}$ & $\mathrm{O}_{1}$ & $\mathrm{C}_{1}$ & $\mathrm{C}_{2}$ & $-166.23(15)$ \\
\hline & & & & \\
\hline
\end{tabular}

Table S13. Hydrogen Atom Coordinates $\left(\AA \times 10^{4}\right)$ and Isotropic Displacement Parameters $\left(\AA^{2} \times 10^{3}\right)$ for $7 b$.

\begin{tabular}{|l|l|l|l|l|}
\hline \multicolumn{1}{|c|}{ Atom } & \multicolumn{1}{c|}{$\boldsymbol{x}$} & \multicolumn{1}{c|}{$\boldsymbol{z}$} & \multicolumn{1}{c|}{ U(eq) } \\
\hline $\mathrm{H}_{16}$ & 1825 & 7988 & 1658 & 23 \\
\hline $\mathrm{H}_{21}$ & 761 & 2678 & 3506 & 26 \\
\hline $\mathrm{H}_{15}$ & 905 & 9351 & 1958 & 26 \\
\hline $\mathrm{H}_{5 \mathrm{~A}}$ & 4429 & 3768 & 2477 & 25 \\
\hline $\mathrm{H}_{5 \mathrm{~B}}$ & 5611 & 4200 & 3364 & 25 \\
\hline $\mathrm{H}_{18}$ & 4691 & 3855 & 4981 & 24 \\
\hline $\mathrm{H}_{19}$ & 4188 & 2491 & 5744 & 25 \\
\hline $\mathrm{H}_{22}$ & 1265 & 4056 & 2774 & 23 \\
\hline $\mathrm{H}_{12}$ & 2726 & 7416 & 5124 & 25 \\
\hline $\mathrm{H}_{13}$ & 1803 & 8775 & 5416 & 29 \\
\hline $\mathrm{H}_{8 \mathrm{~A}}$ & 5749 & 6746 & 2098 & 27 \\
\hline $\mathrm{H}_{8 \mathrm{~B}}$ & 4833 & 6318 & 955 & 27 \\
\hline $\mathrm{H}_{7 \mathrm{~A}}$ & 6780 & 5387 & 2610 & 28 \\
\hline $\mathrm{H}_{7 \mathrm{~B}}$ & 6563 & 5476 & 1246 & 28 \\
\hline
\end{tabular}




\begin{tabular}{|l|l|l|l|l|}
\hline $\mathrm{H}_{6 \mathrm{~A}}$ & 4791 & 4515 & 894 & 28 \\
\hline $\mathrm{H}_{6 \mathrm{~B}}$ & 5989 & 3981 & 1586 & 28 \\
\hline $\mathrm{H}_{14}$ & 855 & 9727 & 3831 & 28 \\
\hline $\mathrm{H}_{23 \mathrm{~A}}$ & -1096 & 6405 & 3264 & 40 \\
\hline $\mathrm{H}_{23 \mathrm{~B}}$ & -112 & 6770 & 4408 & 40 \\
\hline $\mathrm{H}_{23 \mathrm{C}}$ & -480 & 5722 & 4301 & 40 \\
\hline
\end{tabular}

Crystal Data for $\mathrm{C}_{23} \mathrm{H}_{20} \mathrm{BrNO}_{2}(M=422.31 \mathrm{~g} / \mathrm{mol})$ : monoclinic, space group $\mathrm{P} 2{ }_{1} / \mathrm{c}$ (no. 14), $a=$ 11.5469(6) $\AA, b=14.4494(5) \AA, c=11.9365(5) \AA, \beta=108.360(5)^{\circ}, V=1890.18(15) \AA^{3}, Z=4, T=$ $99.97(13) \mathrm{K}, \mu(\mathrm{CuK} \alpha)=3.103 \mathrm{~mm}^{-1}$, Dcalc $=1.484 \mathrm{~g} / \mathrm{cm}^{3}, 15080$ reflections measured $\left(8.068^{\circ} \leq 2 \Theta \leq\right.$ $\left.154.676^{\circ}\right), 3914$ unique $\left(R_{\text {int }}=0.0437, \mathrm{R}_{\text {sigma }}=0.0387\right)$ which were used in all calculations. The final $R_{1}$ was $0.0336(\mathrm{I}>2 \sigma(\mathrm{I}))$ and $w R_{2}$ was 0.0928 (all data).

Details:

\section{Fixed Uiso}

At 1.2 times of: all $\mathrm{C}(\mathrm{H})$ groups, $\mathrm{All} \mathrm{C}(\mathrm{H}, \mathrm{H})$ groups

At 1.5 times of: all $\mathrm{C}(\mathrm{H}, \mathrm{H}, \mathrm{H})$ groups

2a. Secondary $\mathrm{CH}_{2}$ refined with riding coordinates: $\mathrm{C}_{5}\left(\mathrm{H}_{5 \mathrm{~A}}, \mathrm{H}_{5 \mathrm{~B}}\right), \mathrm{C}_{8}\left(\mathrm{H}_{8 \mathrm{~A}}, \mathrm{H}_{8 \mathrm{~B}}\right), \mathrm{C}_{7}\left(\mathrm{H}_{7 \mathrm{~A}}, \mathrm{H}_{7 \mathrm{~B}}\right), \mathrm{C}_{6}\left(\mathrm{H}_{6 \mathrm{~A}}, \mathrm{H}_{6 \mathrm{~B}}\right)$ 2b. Aromatic/amide $\mathrm{H}$ refined with riding coordinates: $\mathrm{C}_{16}\left(\mathrm{H}_{16}\right), \mathrm{C}_{21}\left(\mathrm{H}_{21}\right), \mathrm{C}_{15}\left(\mathrm{H}_{15}\right), \mathrm{C}_{18}\left(\mathrm{H}_{18}\right), \mathrm{C}_{19}\left(\mathrm{H}_{19}\right)$, $\mathrm{C}_{22}\left(\mathrm{H}_{22}\right), \mathrm{C}_{12}\left(\mathrm{H}_{12}\right), \mathrm{C}_{13}\left(\mathrm{H}_{13}\right), \mathrm{C}_{14}\left(\mathrm{H}_{14}\right)$

2c. Idealised Me refined as rotating group: $\mathrm{C}_{23}\left(\mathrm{H}_{23 \mathrm{~A}}, \mathrm{H}_{23 \mathrm{~B}}, \mathrm{H}_{23 \mathrm{C}}\right)$ 
Single crystals of $7 \mathbf{c}$ were obtained by slow evaporation of dichloromethane solution at room temperature. (CCDC 2053527).

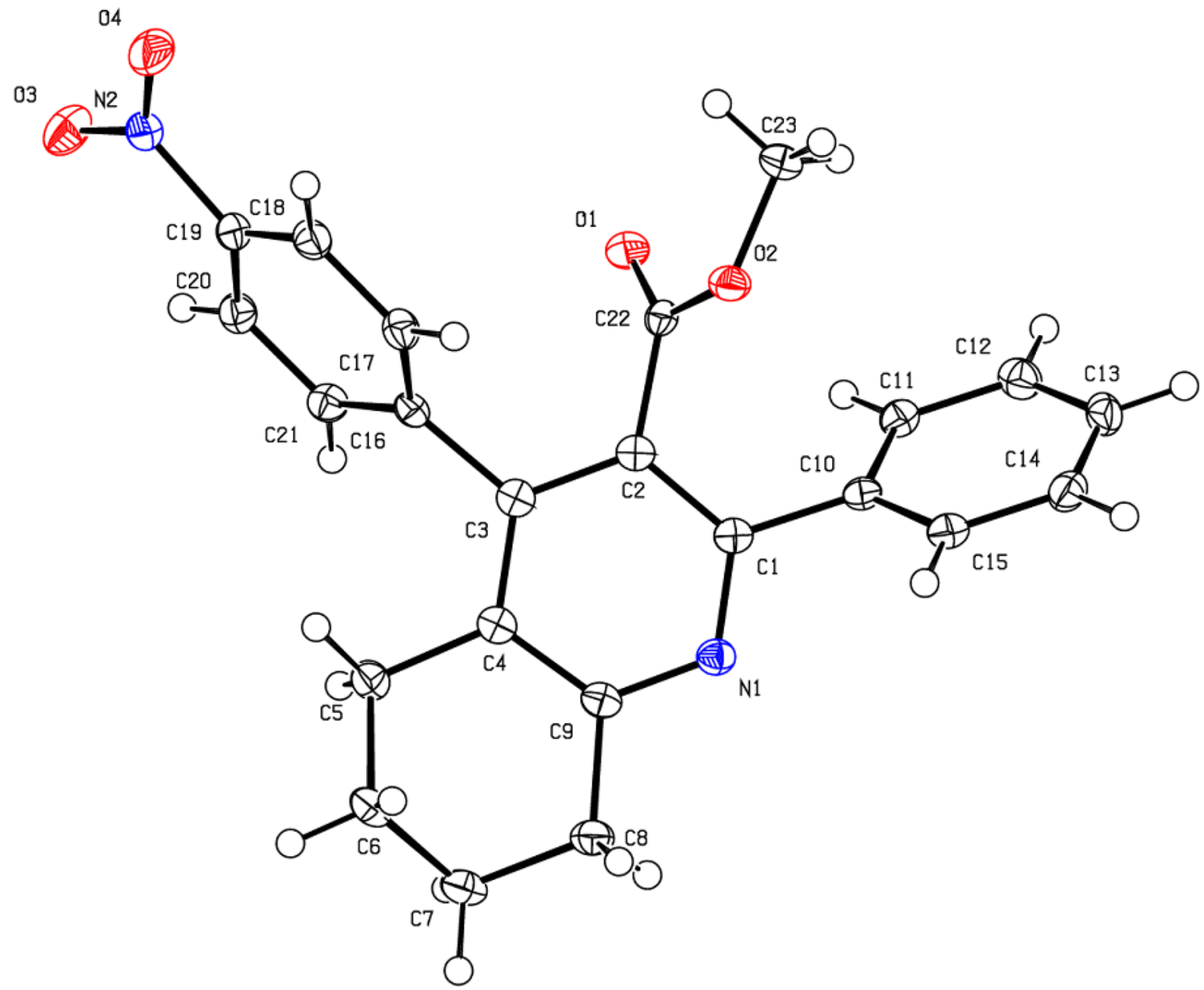

Molecular structure of compound 7c, displacement parameters are drawn at 50\% probability level. 
Table S14. Crystal data and structure refinement for 7c.

\begin{tabular}{|c|c|}
\hline Identification code & 7c (17504_CAT-12.65c) \\
\hline Empirical formula & $\mathrm{C}_{23} \mathrm{H}_{20} \mathrm{~N}_{2} \mathrm{O}_{4}$ \\
\hline Formula weight & 388.41 \\
\hline Temperature/K & $99.96(16)$ \\
\hline Crystal system & monoclinic \\
\hline Space group & $\mathrm{P} 2{ }_{1} / \mathrm{c}$ \\
\hline $\mathrm{a} / \AA$ & $11.6294(4)$ \\
\hline $\mathrm{b} / \AA$ & $14.3841(3)$ \\
\hline $\mathrm{c} / \AA$ & $11.7731(4)$ \\
\hline$\alpha /^{\circ}$ & 90 \\
\hline$\beta /{ }^{\circ}$ & $107.965(3)$ \\
\hline$\gamma /{ }^{\circ}$ & 90 \\
\hline Volume $/ \AA^{3}$ & $1873.38(10)$ \\
\hline Z & 4 \\
\hline$\rho_{\text {calc }} \mathrm{g} / \mathrm{cm}^{3}$ & 1.377 \\
\hline$\mu / \mathrm{mm}^{-1}$ & 0.778 \\
\hline $\mathrm{F}(000)$ & 816.0 \\
\hline Crystal size $/ \mathrm{mm}^{3}$ & $0.20 \times 0.15 \times 0.10$ \\
\hline Radiation & $\mathrm{CuK} \alpha(\lambda=1.54184)$ \\
\hline $2 \Theta$ range for data collection ${ }^{\circ}$ & 10.01 to 139.874 \\
\hline Index ranges & $-14 \leq \mathrm{h} \leq 13,-17 \leq \mathrm{k} \leq 13,-14 \leq 1 \leq 14$ \\
\hline Reflections collected & 13682 \\
\hline Independent reflections & $3455\left[\mathrm{R}_{\text {int }}=0.0355, \mathrm{R}_{\text {sigma }}=0.0279\right]$ \\
\hline Data/restraints/parameters & $3455 / 0 / 263$ \\
\hline Goodness-of-fit on $\mathrm{F}^{2}$ & 1.052 \\
\hline Final $\mathrm{R}$ indexes $[\mathrm{I}>=2 \sigma(\mathrm{I})]$ & $\mathrm{R}_{1}=0.0338, \mathrm{wR}_{2}=0.0864$ \\
\hline Final $\mathrm{R}$ indexes [all data] & $\mathrm{R}_{1}=0.0365, \mathrm{wR}_{2}=0.0882$ \\
\hline Largest diff. peak/hole / e $\AA^{-3}$ & $0.19 /-0.23$ \\
\hline
\end{tabular}

Table S15. Fractional Atomic Coordinates $\left(\times 10^{4}\right)$ and Equivalent Isotropic Displacement Parameters $\left(\AA^{2} \times 10^{3}\right)$ for $7 \mathrm{c} . \mathrm{U}_{\mathrm{eq}}$ is defined as $1 / 3$ of of the trace of the orthogonalised $U_{\mathrm{IJ}}$ tensor.

\begin{tabular}{|c|c|c|c|c|}
\hline Atom & $x$ & $y$ & $\mathbf{Z}$ & $\mathrm{U}(\mathbf{e q})$ \\
\hline $\mathrm{O}_{2}$ & $9333.5(8)$ & $3855.4(6)$ & $6542.8(7)$ & 19.87(19) \\
\hline $\mathrm{O}_{1}$ & $8139.5(8)$ & $4496.6(6)$ & $4840.2(7)$ & $21.6(2)$ \\
\hline $\mathrm{O}_{3}$ & 7473.3(9) & $8818.8(6)$ & $4359.1(8)$ & $30.1(2)$ \\
\hline $\mathrm{O}_{4}$ & 9290.2(9) & $8516.0(6)$ & $5475.4(10)$ & $34.6(2)$ \\
\hline $\mathrm{N}_{1}$ & $6137.5(9)$ & $3134.0(7)$ & $7282.3(8)$ & $17.1(2)$ \\
\hline $\mathrm{N}_{2}$ & $8208.0(10)$ & $8335.5(7)$ & 5091.9(9) & $21.4(2)$ \\
\hline $\mathrm{C}_{19}$ & $7774.7(11)$ & $7490.7(8)$ & $5533.4(10)$ & $18.6(2)$ \\
\hline $\mathrm{C}_{16}$ & $7028.0(10)$ & $5840.5(8)$ & $6270.3(10)$ & $16.4(2)$ \\
\hline $\mathrm{C}_{22}$ & $8284.0(10)$ & $4191.9(7)$ & $5826.9(10)$ & $16.2(2)$ \\
\hline $\mathrm{C}_{10}$ & $7571.3(10)$ & $2375.4(8)$ & $6493.0(10)$ & $16.6(2)$ \\
\hline $\mathrm{C}_{2}$ & $7307.4(10)$ & $4119.5(8)$ & $6415.1(9)$ & $16.2(2)$ \\
\hline $\mathrm{C}_{3}$ & $6677.7(10)$ & $4905.0(8)$ & $6603.4(9)$ & $16.1(2)$ \\
\hline $\mathrm{C}_{4}$ & 5763.4(10) & $4794.2(8)$ & $7140.4(9)$ & $16.4(2)$ \\
\hline $\mathrm{C}_{9}$ & $5551.7(10)$ & $3888.9(8)$ & $7486.5(9)$ & $17.0(2)$ \\
\hline $\mathrm{C}_{20}$ & 6591.3(11) & $7203.8(8)$ & $5003.1(10)$ & $19.2(2)$ \\
\hline $\mathrm{C}_{1}$ & $6988.6(10)$ & $3240.3(8)$ & $6740.4(9)$ & $15.9(2)$ \\
\hline $\mathrm{C}_{11}$ & $7568.7(11)$ & $2159.2(8)$ & $5335.1(10)$ & $18.4(2)$ \\
\hline $\mathrm{C}_{17}$ & $8197.2(11)$ & $6172.5(8)$ & $6818.2(10)$ & $19.0(2)$ \\
\hline
\end{tabular}




\begin{tabular}{|l|l|l|l|l|}
\hline $\mathrm{C}_{5}$ & $5047.5(11)$ & $5609.4(8)$ & $7372.7(10)$ & $19.5(2)$ \\
\hline $\mathrm{C}_{21}$ & $6223.0(11)$ & $6373.7(8)$ & $5384.3(10)$ & $18.6(2)$ \\
\hline $\mathrm{C}_{6}$ & $4545.4(11)$ & $5410.6(9)$ & $8402.3(11)$ & $21.7(3)$ \\
\hline $\mathrm{C}_{14}$ & $8663.5(11)$ & $958.2(8)$ & $7201.3(11)$ & $21.2(3)$ \\
\hline $\mathrm{C}_{18}$ & $8581.2(11)$ & $6998.8(8)$ & $6450.9(11)$ & $20.2(2)$ \\
\hline $\mathrm{C}_{8}$ & $4648.6(11)$ & $3695.7(8)$ & $8146.7(11)$ & $21.2(3)$ \\
\hline $\mathrm{C}_{15}$ & $8109.4(11)$ & $1759.8(8)$ & $7419.8(10)$ & $19.1(2)$ \\
\hline $\mathrm{C}_{23}$ & $10266.3(11)$ & $3712.5(9)$ & $5983.3(12)$ & $24.0(3)$ \\
\hline $\mathrm{C}_{13}$ & $8685.7(11)$ & $755.2(8)$ & $6054.0(11)$ & $22.7(3)$ \\
\hline $\mathrm{C}_{7}$ & $3813.7(11)$ & $4512.1(9)$ & $8149.4(11)$ & $22.6(3)$ \\
\hline $\mathrm{C}_{12}$ & $8124.3(12)$ & $1353.0(8)$ & $5119.2(11)$ & $22.4(3)$ \\
\hline
\end{tabular}

Table S16. Anisotropic Displacement Parameters $\left(\AA^{2} \times 10^{3}\right)$ for $7 \mathrm{c}$. The Anisotropic displacement factor exponent takes the form: $-2 \pi^{2}\left[h^{2} a *{ }^{2} U_{11}+2 h k a * b * U_{12}+\ldots\right]$.

\begin{tabular}{|l|l|l|l|l|l|l|}
\hline \multicolumn{1}{|c|}{ Atom } & \multicolumn{1}{|c|}{$\mathbf{U}_{\mathbf{1 1}}$} & \multicolumn{1}{c|}{$\mathbf{U}_{\mathbf{2 2}}$} & \multicolumn{1}{c|}{$\mathbf{U}_{\mathbf{3 3}}$} & \multicolumn{1}{c|}{$\mathbf{U}_{\mathbf{2 3}}$} & \multicolumn{1}{c|}{$\mathbf{U}_{\mathbf{1 3}}$} & \multicolumn{1}{c|}{$\mathbf{U}_{\mathbf{1 2}}$} \\
\hline $\mathrm{O}_{2}$ & $16.3(4)$ & $22.4(4)$ & $22.6(4)$ & $1.4(3)$ & $8.3(3)$ & $-0.3(3)$ \\
\hline $\mathrm{O}_{1}$ & $24.1(5)$ & $23.1(4)$ & $20.2(4)$ & $2.4(3)$ & $10.7(3)$ & $-0.2(3)$ \\
\hline $\mathrm{O}_{3}$ & $33.1(5)$ & $25.4(5)$ & $29.7(5)$ & $8.8(4)$ & $6.8(4)$ & $-2.0(4)$ \\
\hline $\mathrm{O}_{4}$ & $21.6(5)$ & $23.4(5)$ & $60.6(7)$ & $5.6(4)$ & $15.2(5)$ & $-2.5(4)$ \\
\hline $\mathrm{N}_{1}$ & $16.5(5)$ & $18.9(5)$ & $16.0(4)$ & $1.3(4)$ & $5.0(4)$ & $0.0(4)$ \\
\hline $\mathrm{N}_{2}$ & $23.3(6)$ & $17.0(5)$ & $27.3(5)$ & $-2.5(4)$ & $12.7(4)$ & $-0.6(4)$ \\
\hline $\mathrm{C}_{19}$ & $22.4(6)$ & $14.5(5)$ & $21.8(5)$ & $-2.3(4)$ & $10.9(5)$ & $-0.5(4)$ \\
\hline $\mathrm{C}_{16}$ & $17.9(6)$ & $16.8(5)$ & $16.5(5)$ & $-2.3(4)$ & $8.4(4)$ & $1.1(4)$ \\
\hline $\mathrm{C}_{22}$ & $18.4(6)$ & $12.3(5)$ & $18.9(5)$ & $-2.5(4)$ & $7.3(4)$ & $-2.6(4)$ \\
\hline $\mathrm{C}_{10}$ & $13.0(5)$ & $16.9(5)$ & $20.4(5)$ & $-0.4(4)$ & $5.6(4)$ & $-3.2(4)$ \\
\hline $\mathrm{C}_{2}$ & $15.1(5)$ & $18.6(6)$ & $14.2(5)$ & $-0.4(4)$ & $3.8(4)$ & $-1.0(4)$ \\
\hline $\mathrm{C}_{3}$ & $15.3(6)$ & $18.7(5)$ & $12.7(5)$ & $-1.0(4)$ & $2.0(4)$ & $-0.5(4)$ \\
\hline $\mathrm{C}_{4}$ & $14.7(5)$ & $19.7(6)$ & $13.6(5)$ & $-1.0(4)$ & $2.6(4)$ & $-0.2(4)$ \\
\hline $\mathrm{C}_{9}$ & $14.5(6)$ & $21.4(6)$ & $14.8(5)$ & $-0.3(4)$ & $4.1(4)$ & $0.0(4)$ \\
\hline $\mathrm{C}_{20}$ & $20.7(6)$ & $18.7(5)$ & $18.2(5)$ & $0.5(4)$ & $6.0(4)$ & $2.3(4)$ \\
\hline $\mathrm{C}_{1}$ & $14.4(5)$ & $19.0(6)$ & $13.7(5)$ & $0.7(4)$ & $3.2(4)$ & $-0.7(4)$ \\
\hline $\mathrm{C}_{11}$ & $17.6(6)$ & $18.6(5)$ & $18.6(5)$ & $1.0(4)$ & $5.0(4)$ & $-1.6(4)$ \\
\hline $\mathrm{C}_{17}$ & $16.8(6)$ & $18.4(6)$ & $20.7(6)$ & $-0.3(4)$ & $4.3(4)$ & $2.4(4)$ \\
\hline $\mathrm{C}_{5}$ & $18.5(6)$ & $20.3(6)$ & $19.9(5)$ & $0.3(4)$ & $6.3(4)$ & $2.5(4)$ \\
\hline $\mathrm{C}_{21}$ & $16.8(6)$ & $20.5(6)$ & $18.2(5)$ & $-1.7(4)$ & $4.9(4)$ & $-1.4(4)$ \\
\hline $\mathrm{C}_{6}$ & $19.8(6)$ & $25.3(6)$ & $22.0(6)$ & $-0.9(5)$ & $9.5(5)$ & $4.5(5)$ \\
\hline $\mathrm{C}_{14}$ & $16.8(6)$ & $18.3(6)$ & $27.3(6)$ & $4.8(5)$ & $4.9(5)$ & $-0.1(4)$ \\
\hline $\mathrm{C}_{18}$ & $16.4(6)$ & $18.7(6)$ & $26.0(6)$ & $-4.4(5)$ & $6.9(5)$ & $-1.3(4)$ \\
\hline $\mathrm{C}_{8}$ & $20.5(6)$ & $23.9(6)$ & $21.9(6)$ & $2.0(5)$ & $10.5(5)$ & $-0.5(5)$ \\
\hline $\mathrm{C}_{15}$ & $17.3(6)$ & $20.9(6)$ & $19.8(5)$ & $1.2(4)$ & $6.9(4)$ & $-2.2(4)$ \\
\hline $\mathrm{C}_{23}$ & $17.7(6)$ & $24.2(6)$ & $34.0(7)$ & $0.5(5)$ & $13.7(5)$ & $0.7(5)$ \\
\hline $\mathrm{C}_{13}$ & $17.3(6)$ & $16.4(5)$ & $34.7(7)$ & $-4.6(5)$ & $8.4(5)$ & $-0.9(4)$ \\
\hline $\mathrm{C}_{7}$ & $17.9(6)$ & $29.1(6)$ & $23.2(6)$ & $1.4(5)$ & $10.1(5)$ & $1.6(5)$ \\
\hline $\mathrm{C}_{12}$ & $21.2(6)$ & $23.6(6)$ & $23.3(6)$ & $-5.5(5)$ & $8.1(5)$ & $-2.5(5)$ \\
\hline
\end{tabular}


Table S17. Bond Lengths for 7c.

\begin{tabular}{|c|c|c|}
\hline Atom & Atom & Length/Å \\
\hline $\mathrm{O}_{2}$ & $\mathrm{C}_{22}$ & $1.3415(15)$ \\
\hline $\mathrm{O}_{2}$ & $\mathrm{C}_{23}$ & $1.4469(14)$ \\
\hline $\mathrm{O}_{1}$ & $\mathrm{C}_{22}$ & $1.2039(14)$ \\
\hline $\mathrm{O}_{3}$ & $\mathrm{~N}_{2}$ & $1.2259(14)$ \\
\hline $\mathrm{O}_{4}$ & $\mathrm{~N}_{2}$ & $1.2266(15)$ \\
\hline $\mathrm{N}_{1}$ & $\mathrm{C}_{9}$ & $1.3423(15)$ \\
\hline $\mathrm{N}_{1}$ & $\mathrm{C}_{1}$ & $1.3415(15)$ \\
\hline $\mathrm{N}_{2}$ & $\mathrm{C}_{19}$ & $1.4706(15)$ \\
\hline $\mathrm{C}_{19}$ & $\mathrm{C}_{20}$ & $1.3871(18)$ \\
\hline $\mathrm{C}_{19}$ & $\mathrm{C}_{18}$ & $1.3864(17)$ \\
\hline $\mathrm{C}_{16}$ & $\mathrm{C}_{3}$ & $1.4930(16)$ \\
\hline $\mathrm{C}_{16}$ & $\mathrm{C}_{17}$ & $1.3972(17)$ \\
\hline $\mathrm{C}_{16}$ & $\mathrm{C}_{21}$ & $1.3965(16)$ \\
\hline $\mathrm{C}_{22}$ & $\mathrm{C}_{2}$ & $1.5052(15)$ \\
\hline $\mathrm{C}_{10}$ & $\mathrm{C}_{1}$ & $1.4878(16)$ \\
\hline $\mathrm{C}_{10}$ & $\mathrm{C}_{11}$ & $1.3974(16)$ \\
\hline
\end{tabular}

\begin{tabular}{|l|l|l|}
\hline Atom & Atom & Length $/ \AA$ \\
\hline $\mathrm{C}_{10}$ & $\mathrm{C}_{15}$ & $1.3945(16)$ \\
\hline $\mathrm{C}_{2}$ & $\mathrm{C}_{3}$ & $1.4006(16)$ \\
\hline $\mathrm{C}_{2}$ & $\mathrm{C}_{1}$ & $1.4039(16)$ \\
\hline $\mathrm{C}_{3}$ & $\mathrm{C}_{4}$ & $1.4037(16)$ \\
\hline $\mathrm{C}_{4}$ & $\mathrm{C}_{9}$ & $1.4086(16)$ \\
\hline $\mathrm{C}_{4}$ & $\mathrm{C}_{5}$ & $1.5110(16)$ \\
\hline $\mathrm{C}_{9}$ & $\mathrm{C}_{8}$ & $1.5126(16)$ \\
\hline $\mathrm{C}_{20}$ & $\mathrm{C}_{21}$ & $1.3889(17)$ \\
\hline $\mathrm{C}_{11}$ & $\mathrm{C}_{12}$ & $1.3886(17)$ \\
\hline $\mathrm{C}_{17}$ & $\mathrm{C}_{18}$ & $1.3850(17)$ \\
\hline $\mathrm{C}_{5}$ & $\mathrm{C}_{6}$ & $1.5259(16)$ \\
\hline $\mathrm{C}_{6}$ & $\mathrm{C}_{7}$ & $1.5254(18)$ \\
\hline $\mathrm{C}_{14}$ & $\mathrm{C}_{15}$ & $1.3831(17)$ \\
\hline $\mathrm{C}_{14}$ & $\mathrm{C}_{13}$ & $1.3899(18)$ \\
\hline $\mathrm{C}_{8}$ & $\mathrm{C}_{7}$ & $1.5243(17)$ \\
\hline $\mathrm{C}_{13}$ & $\mathrm{C}_{12}$ & $1.3906(18)$ \\
\hline
\end{tabular}

Table S18. Bond Angles for 7c.

\begin{tabular}{|l|l|l|l|}
\hline Atom & Atom & Atom & \multicolumn{1}{|c|}{ Angle/ } \\
\hline $\mathrm{C}_{22}$ & $\mathrm{O}_{2}$ & $\mathrm{C}_{23}$ & $115.46(9)$ \\
\hline $\mathrm{C}_{1}$ & $\mathrm{~N}_{1}$ & $\mathrm{C}_{9}$ & $118.86(10)$ \\
\hline $\mathrm{O}_{3}$ & $\mathrm{~N}_{2}$ & $\mathrm{O}_{4}$ & $123.52(10)$ \\
\hline $\mathrm{O}_{3}$ & $\mathrm{~N}_{2}$ & $\mathrm{C}_{19}$ & $118.48(10)$ \\
\hline $\mathrm{O}_{4}$ & $\mathrm{~N}_{2}$ & $\mathrm{C}_{19}$ & $117.99(10)$ \\
\hline $\mathrm{C}_{20}$ & $\mathrm{C}_{19}$ & $\mathrm{~N}_{2}$ & $119.17(10)$ \\
\hline $\mathrm{C}_{18}$ & $\mathrm{C}_{19}$ & $\mathrm{~N}_{2}$ & $118.28(11)$ \\
\hline $\mathrm{C}_{18}$ & $\mathrm{C}_{19}$ & $\mathrm{C}_{20}$ & $122.53(11)$ \\
\hline $\mathrm{C}_{17}$ & $\mathrm{C}_{16}$ & $\mathrm{C}_{3}$ & $119.50(10)$ \\
\hline $\mathrm{C}_{21}$ & $\mathrm{C}_{16}$ & $\mathrm{C}_{3}$ & $121.12(10)$ \\
\hline $\mathrm{C}_{21}$ & $\mathrm{C}_{16}$ & $\mathrm{C}_{17}$ & $119.33(11)$ \\
\hline $\mathrm{O}_{2}$ & $\mathrm{C}_{22}$ & $\mathrm{C}_{2}$ & $110.82(9)$ \\
\hline $\mathrm{O}_{1}$ & $\mathrm{C}_{22}$ & $\mathrm{O}_{2}$ & $124.52(10)$ \\
\hline $\mathrm{O}_{1}$ & $\mathrm{C}_{22}$ & $\mathrm{C}_{2}$ & $124.65(10)$ \\
\hline $\mathrm{C}_{11}$ & $\mathrm{C}_{10}$ & $\mathrm{C}_{1}$ & $120.86(10)$ \\
\hline $\mathrm{C}_{15}$ & $\mathrm{C}_{10}$ & $\mathrm{C}_{1}$ & $119.96(10)$ \\
\hline $\mathrm{C}_{15}$ & $\mathrm{C}_{10}$ & $\mathrm{C}_{11}$ & $119.17(11)$ \\
\hline $\mathrm{C}_{3}$ & $\mathrm{C}_{2}$ & $\mathrm{C}_{22}$ & $121.50(10)$ \\
\hline $\mathrm{C}_{3}$ & $\mathrm{C}_{2}$ & $\mathrm{C}_{1}$ & $119.24(10)$ \\
\hline $\mathrm{C}_{1}$ & $\mathrm{C}_{2}$ & $\mathrm{C}_{22}$ & $119.21(10)$ \\
\hline $\mathrm{C}_{2}$ & $\mathrm{C}_{3}$ & $\mathrm{C}_{16}$ & $119.15(10)$ \\
\hline $\mathrm{C}_{2}$ & $\mathrm{C}_{3}$ & $\mathrm{C}_{4}$ & $119.04(10)$ \\
\hline $\mathrm{C}_{4}$ & $\mathrm{C}_{3}$ & $\mathrm{C}_{16}$ & $121.77(10)$ \\
\hline & & & \\
\hline
\end{tabular}

\begin{tabular}{|l|l|l|c|}
\hline Atom & Atom & Atom & \multicolumn{1}{|c|}{ Angle $^{\circ}$} \\
\hline $\mathrm{C}_{3}$ & $\mathrm{C}_{4}$ & $\mathrm{C}_{9}$ & $117.35(10)$ \\
\hline $\mathrm{C}_{3}$ & $\mathrm{C}_{4}$ & $\mathrm{C}_{5}$ & $121.98(10)$ \\
\hline $\mathrm{C}_{9}$ & $\mathrm{C}_{4}$ & $\mathrm{C}_{5}$ & $120.65(10)$ \\
\hline $\mathrm{N}_{1}$ & $\mathrm{C}_{9}$ & $\mathrm{C}_{4}$ & $123.53(10)$ \\
\hline $\mathrm{N}_{1}$ & $\mathrm{C}_{9}$ & $\mathrm{C}_{8}$ & $114.49(10)$ \\
\hline $\mathrm{C}_{4}$ & $\mathrm{C}_{9}$ & $\mathrm{C}_{8}$ & $121.97(10)$ \\
\hline $\mathrm{C}_{19}$ & $\mathrm{C}_{20}$ & $\mathrm{C}_{21}$ & $118.19(11)$ \\
\hline $\mathrm{N}_{1}$ & $\mathrm{C}_{1}$ & $\mathrm{C}_{10}$ & $116.43(10)$ \\
\hline $\mathrm{N}_{1}$ & $\mathrm{C}_{1}$ & $\mathrm{C}_{2}$ & $121.87(10)$ \\
\hline $\mathrm{C}_{2}$ & $\mathrm{C}_{1}$ & $\mathrm{C}_{10}$ & $121.70(10)$ \\
\hline $\mathrm{C}_{12}$ & $\mathrm{C}_{11}$ & $\mathrm{C}_{10}$ & $120.22(11)$ \\
\hline $\mathrm{C}_{18}$ & $\mathrm{C}_{17}$ & $\mathrm{C}_{16}$ & $120.72(11)$ \\
\hline $\mathrm{C}_{4}$ & $\mathrm{C}_{5}$ & $\mathrm{C}_{6}$ & $111.44(10)$ \\
\hline $\mathrm{C}_{20}$ & $\mathrm{C}_{21}$ & $\mathrm{C}_{16}$ & $120.73(11)$ \\
\hline $\mathrm{C}_{7}$ & $\mathrm{C}_{6}$ & $\mathrm{C}_{5}$ & $109.32(10)$ \\
\hline $\mathrm{C}_{15}$ & $\mathrm{C}_{14}$ & $\mathrm{C}_{13}$ & $120.35(11)$ \\
\hline $\mathrm{C}_{17}$ & $\mathrm{C}_{18}$ & $\mathrm{C}_{19}$ & $118.38(11)$ \\
\hline $\mathrm{C}_{9}$ & $\mathrm{C}_{8}$ & $\mathrm{C}_{7}$ & $113.78(10)$ \\
\hline $\mathrm{C}_{14}$ & $\mathrm{C}_{15}$ & $\mathrm{C}_{10}$ & $120.41(11)$ \\
\hline $\mathrm{C}_{14}$ & $\mathrm{C}_{13}$ & $\mathrm{C}_{12}$ & $119.62(11)$ \\
\hline $\mathrm{C}_{8}$ & $\mathrm{C}_{7}$ & $\mathrm{C}_{6}$ & $109.62(10)$ \\
\hline $\mathrm{C}_{11}$ & $\mathrm{C}_{12}$ & $\mathrm{C}_{13}$ & $120.19(11)$ \\
\hline
\end{tabular}


Table S19. Torsion Angles for 7c.

\begin{tabular}{|c|c|c|c|c|}
\hline A & B & C & D & Angle $/{ }^{\circ}$ \\
\hline $\mathrm{O}_{2}$ & $\mathrm{C}_{22}$ & $\mathrm{C}_{2}$ & $\mathrm{C}_{3}$ & $122.20(11)$ \\
\hline $\mathrm{O}_{2}$ & $\mathrm{C}_{22}$ & $\mathrm{C}_{2}$ & $\mathrm{C}_{1}$ & $60.30(13)$ \\
\hline $\mathrm{O}_{1}$ & $\mathrm{C}_{22}$ & $\mathrm{C}_{2}$ & $\mathrm{C}_{3}$ & $58.91(16)$ \\
\hline $\mathrm{O}_{1}$ & $\mathrm{C}_{22}$ & $\mathrm{C}_{2}$ & $\mathrm{C}_{1}$ & $118.59(12)$ \\
\hline $\mathrm{O}_{3}$ & $\mathrm{~N}_{2}$ & $\mathrm{C}_{19}$ & $\mathrm{C}_{20}$ & $8.09(15)$ \\
\hline $\mathrm{O}_{3}$ & $\mathrm{~N}_{2}$ & $\mathrm{C}_{19}$ & $\mathrm{C}_{18}$ & $-173.40(10)$ \\
\hline $\mathrm{O}_{4}$ & $\mathrm{~N}_{2}$ & $\mathrm{C}_{19}$ & $\mathrm{C} 20$ & $0(11)$ \\
\hline $\mathrm{O}_{4}$ & $\mathrm{~N}_{2}$ & $\mathrm{C}_{19}$ & $\mathrm{C}_{18}$ & 6.91 \\
\hline $\mathrm{N}_{1}$ & $\mathrm{C}_{9}$ & $\mathrm{C}_{8}$ & $\mathrm{C}_{7}$ & -168 \\
\hline $\mathrm{N}_{2}$ & $\mathrm{C}_{19}$ & $\mathrm{C}_{20}$ & $\mathrm{C}_{21}$ & 176. \\
\hline $\mathrm{N}_{2}$ & $\mathrm{C}_{19}$ & $\mathrm{C}_{18}$ & & (10) \\
\hline $\mathrm{C}_{19}$ & $\mathrm{C}_{20}$ & $\mathrm{C}_{21}$ & $\mathrm{C}_{16}$ & -0.5 \\
\hline $\mathrm{C}_{16}$ & $\mathrm{C}_{3}$ & $\mathrm{C}_{4}$ & $\mathrm{C}_{9}$ & $1(10)$ \\
\hline $\mathrm{C}_{16}$ & $\mathrm{C}_{3}$ & $\mathrm{C}_{4}$ & $\mathrm{C}_{5}$ & 2.2 \\
\hline $\mathrm{C}_{16}$ & $\mathrm{C}_{17}$ & $\mathrm{C}_{18}$ & $\mathrm{C}_{19}$ & 0.46 \\
\hline $\mathrm{C}_{22}$ & $\mathrm{C}_{2}$ & $\mathrm{C}_{3}$ & $\mathrm{C}_{16}$ & -3.22 \\
\hline $\mathrm{C}_{22}$ & $\mathrm{C}_{2}$ & $\mathrm{C}_{3}$ & $\mathrm{C}_{4}$ & 179. \\
\hline $\mathrm{C}_{22}$ & $\mathrm{C}_{2}$ & $\mathrm{C}_{1}$ & $\mathrm{~N}_{1}$ & 179. \\
\hline $\mathrm{C}_{22}$ & $\mathrm{C}_{2}$ & $\mathrm{C}_{1}$ & & -1.4 \\
\hline $\mathrm{C}_{10}$ & $\mathrm{C}_{11}$ & $\mathrm{C}_{12}$ & $\mathrm{C}_{13}$ & 0.06 \\
\hline $\mathrm{C}_{2}$ & $\mathrm{C}_{3}$ & $\mathrm{C}_{4}$ & $\mathrm{C}_{9}$ & $1.43(1$ \\
\hline $\mathrm{C}_{2}$ & $\mathrm{C}_{3}$ & $\mathrm{C}_{4}$ & $\mathrm{C}_{5}$ & 179. \\
\hline $\mathrm{C}_{3}$ & $\mathrm{C}_{16}$ & $\mathrm{C}_{17}$ & & 174. \\
\hline $\mathrm{C}_{3}$ & $\mathrm{C}_{16}$ & & & -174 \\
\hline $\mathrm{C}_{3}$ & $\mathrm{C}_{2}$ & $\mathrm{C}_{1}$ & $\mathrm{~N}_{1}$ & $-3.40(16)$ \\
\hline $\mathrm{C}_{3}$ & $\mathrm{C}_{2}$ & $\mathrm{C}_{1}$ & $\mathrm{C}_{10}$ & $176.10(10)$ \\
\hline $\mathrm{C}_{3}$ & $\mathrm{C}_{4}$ & $\mathrm{C}_{9}$ & $\mathrm{~N}_{1}$ & $-3.00(16)$ \\
\hline $\mathrm{C}_{3}$ & $\mathrm{C}_{4}$ & $\mathrm{C}_{9}$ & & $175.83(10)$ \\
\hline $\mathrm{C}_{3}$ & $\mathrm{C}_{4}$ & $\mathrm{C}_{5}$ & $\mathrm{C}_{6}$ & $-154.74(10)$ \\
\hline $\mathrm{C}_{4}$ & $\mathrm{C}_{9}$ & $\mathrm{C}_{8}$ & $\mathrm{C}_{7}$ & $12.88(16)$ \\
\hline $\mathrm{C}_{4}$ & $\mathrm{C}_{5}$ & $\mathrm{C}_{6}$ & $\mathrm{C}_{7}$ & $-54.63(13)$ \\
\hline $\mathrm{C}_{9}$ & $\mathrm{~N}_{1}$ & $\mathrm{C}_{1}$ & $\mathrm{C}_{10}$ & $-177.57(9)$ \\
\hline
\end{tabular}

\begin{tabular}{|c|l|l|l|l|}
\hline $\mathbf{A}$ & \multicolumn{1}{|c|}{$\mathbf{B}$} & \multicolumn{1}{|c|}{$\mathbf{C}$} & \multicolumn{1}{|c|}{$\mathbf{D}$} & \multicolumn{1}{|c|}{ Angle ${ }^{\circ}$} \\
\hline $\mathrm{C}_{9}$ & $\mathrm{~N}_{1}$ & $\mathrm{C}_{1}$ & $\mathrm{C}_{2}$ & $1.96(16)$ \\
\hline $\mathrm{C}_{9}$ & $\mathrm{C}_{4}$ & $\mathrm{C}_{5}$ & $\mathrm{C}_{6}$ & $23.71(15)$ \\
\hline $\mathrm{C}_{9}$ & $\mathrm{C}_{8}$ & $\mathrm{C}_{7}$ & $\mathrm{C}_{6}$ & $-43.56(13)$ \\
\hline $\mathrm{C}_{20}$ & $\mathrm{C}_{19}$ & $\mathrm{C}_{18}$ & $\mathrm{C}_{17}$ & $2.38(17)$ \\
\hline $\mathrm{C}_{1}$ & $\mathrm{~N}_{1}$ & $\mathrm{C}_{9}$ & $\mathrm{C}_{4}$ & $1.31(16)$ \\
\hline $\mathrm{C}_{1}$ & $\mathrm{~N}_{1}$ & $\mathrm{C}_{9}$ & $\mathrm{C}_{8}$ & $-177.60(10)$ \\
\hline $\mathrm{C}_{1}$ & $\mathrm{C}_{10}$ & $\mathrm{C}_{11}$ & $\mathrm{C}_{12}$ & $179.50(11)$ \\
\hline $\mathrm{C}_{1}$ & $\mathrm{C}_{10}$ & $\mathrm{C}_{15}$ & $\mathrm{C}_{14}$ & $-179.38(10)$ \\
\hline $\mathrm{C}_{1}$ & $\mathrm{C}_{2}$ & $\mathrm{C}_{3}$ & $\mathrm{C}_{16}$ & $179.27(10)$ \\
\hline $\mathrm{C}_{1}$ & $\mathrm{C}_{2}$ & $\mathrm{C}_{3}$ & $\mathrm{C}_{4}$ & $1.57(16)$ \\
\hline $\mathrm{C}_{11}$ & $\mathrm{C}_{10}$ & $\mathrm{C}_{1}$ & $\mathrm{~N}_{1}$ & $124.05(11)$ \\
\hline $\mathrm{C}_{11}$ & $\mathrm{C}_{10}$ & $\mathrm{C}_{1}$ & $\mathrm{C}_{2}$ & $-55.48(15)$ \\
\hline $\mathrm{C}_{11}$ & $\mathrm{C}_{10}$ & $\mathrm{C}_{15}$ & $\mathrm{C}_{14}$ & $1.80(17)$ \\
\hline $\mathrm{C}_{17}$ & $\mathrm{C}_{16}$ & $\mathrm{C}_{3}$ & $\mathrm{C}_{2}$ & $-60.62(14)$ \\
\hline $\mathrm{C}_{17}$ & $\mathrm{C}_{16}$ & $\mathrm{C}_{3}$ & $\mathrm{C}_{4}$ & $117.02(12)$ \\
\hline $\mathrm{C}_{17}$ & $\mathrm{C}_{16}$ & $\mathrm{C}_{21}$ & $\mathrm{C}_{20}$ & $3.26(16)$ \\
\hline $\mathrm{C}_{5}$ & $\mathrm{C}_{4}$ & $\mathrm{C}_{9}$ & $\mathrm{~N}_{1}$ & $178.48(10)$ \\
\hline $\mathrm{C}_{5}$ & $\mathrm{C}_{4}$ & $\mathrm{C}_{9}$ & $\mathrm{C}_{8}$ & $-2.69(16)$ \\
\hline $\mathrm{C}_{5}$ & $\mathrm{C}_{6}$ & $\mathrm{C}_{7}$ & $\mathrm{C}_{8}$ & $65.51(12)$ \\
\hline $\mathrm{C}_{21}$ & $\mathrm{C}_{16}$ & $\mathrm{C}_{3}$ & $\mathrm{C}_{2}$ & $116.68(12)$ \\
\hline $\mathrm{C}_{21}$ & $\mathrm{C}_{16}$ & $\mathrm{C}_{3}$ & $\mathrm{C}_{4}$ & $-65.68(14)$ \\
\hline $\mathrm{C}_{21}$ & $\mathrm{C}_{16}$ & $\mathrm{C}_{17}$ & $\mathrm{C}_{18}$ & $-3.22(17)$ \\
\hline $\mathrm{C}_{14}$ & $\mathrm{C}_{13}$ & $\mathrm{C}_{12}$ & $\mathrm{C}_{11}$ & $1.47(18)$ \\
\hline $\mathrm{C}_{18}$ & $\mathrm{C}_{19}$ & $\mathrm{C}_{20}$ & $\mathrm{C}_{21}$ & $-2.33(17)$ \\
\hline $\mathrm{C}_{15}$ & $\mathrm{C}_{10}$ & $\mathrm{C}_{1}$ & $\mathrm{~N}_{1}$ & $-54.75(14)$ \\
\hline $\mathrm{C}_{15}$ & $\mathrm{C}_{10}$ & $\mathrm{C}_{1}$ & $\mathrm{C}_{2}$ & $125.72(12)$ \\
\hline $\mathrm{C}_{15}$ & $\mathrm{C}_{10}$ & $\mathrm{C}_{11}$ & $\mathrm{C}_{12}$ & $-1.69(17)$ \\
\hline $\mathrm{C}_{15}$ & $\mathrm{C}_{14}$ & $\mathrm{C}_{13}$ & $\mathrm{C}_{12}$ & $-1.37(18)$ \\
\hline $\mathrm{C}_{23}$ & $\mathrm{O}_{2}$ & $\mathrm{C}_{22}$ & $\mathrm{O}_{1}$ & $-11.10(15)$ \\
\hline $\mathrm{C}_{23}$ & $\mathrm{O}_{2}$ & $\mathrm{C}_{22}$ & $\mathrm{C}_{2}$ & $167.79(9)$ \\
\hline $\mathrm{C}_{13}$ & $\mathrm{C}_{14}$ & $\mathrm{C}_{15}$ & $\mathrm{C}_{10}$ & $-0.27(18)$ \\
\hline & & & & \\
\hline
\end{tabular}

Table S20. Hydrogen Atom Coordinates $\left(\AA \times 10^{4}\right)$ and Isotropic Displacement Parameters $\left(\AA^{2} \times 10^{3}\right)$ for $7 \mathrm{c}$.

\begin{tabular}{|l|l|l|l|l|}
\hline \multicolumn{1}{|c|}{ Atom } & \multicolumn{1}{c|}{$\boldsymbol{x}$} & \multicolumn{1}{c|}{$\boldsymbol{y}$} & \multicolumn{1}{c|}{ U(eq) } \\
\hline $\mathrm{H}_{20}$ & 6059 & 7558 & 4408 & 23 \\
\hline $\mathrm{H}_{11}$ & 7194 & 2557 & 4707 & 22 \\
\hline $\mathrm{H}_{17}$ & 8723 & 5835 & 7436 & 23 \\
\hline $\mathrm{H}_{5 \mathrm{~A}}$ & 4385 & 5743 & 6657 & 23 \\
\hline $\mathrm{H}_{5 \mathrm{~B}}$ & 5564 & 6154 & 7561 & 23 \\
\hline $\mathrm{H}_{21}$ & 5432 & 6171 & 5046 & 22 \\
\hline $\mathrm{H}_{6 \mathrm{~A}}$ & 5205 & 5351 & 9141 & 26 \\
\hline $\mathrm{H}_{6 \mathrm{~B}}$ & 4034 & 5921 & 8489 & 26 \\
\hline $\mathrm{H}_{14}$ & 9023 & 553 & 7825 & 25 \\
\hline $\mathrm{H}_{18}$ & 9361 & 7218 & 6811 & 24 \\
\hline $\mathrm{H}_{8 \mathrm{~A}}$ & 5085 & 3533 & 8966 & 25 \\
\hline $\mathrm{H}_{8 \mathrm{~B}}$ & 4161 & 3164 & 7783 & 25 \\
\hline
\end{tabular}




\begin{tabular}{|l|l|l|l|l|}
\hline $\mathrm{H}_{15}$ & 8096 & 1889 & 8189 & 23 \\
\hline $\mathrm{H}_{23 \mathrm{~A}}$ & 10017 & 3234 & 5390 & 36 \\
\hline $\mathrm{H}_{23 \mathrm{~B}}$ & 11002 & 3529 & 6577 & 36 \\
\hline $\mathrm{H}_{23 \mathrm{C}}$ & 10398 & 4280 & 5613 & 36 \\
\hline $\mathrm{H}_{13}$ & 9074 & 223 & 5912 & 27 \\
\hline $\mathrm{H}_{7 \mathrm{~A}}$ & 3420 & 4416 & 8756 & 27 \\
\hline $\mathrm{H}_{7 \mathrm{~B}}$ & 3193 & 4555 & 7380 & 27 \\
\hline $\mathrm{H}_{12}$ & 8121 & 1212 & 4347 & 27 \\
\hline
\end{tabular}

Crystal Data for $\mathrm{C}_{23} \mathrm{H}_{20} \mathrm{~N}_{2} \mathrm{O}_{4}(M=388.41 \mathrm{~g} / \mathrm{mol})$ : monoclinic, space group $\mathrm{P} 2{ }_{1} / \mathrm{c}$ (no. 14), $a=$ 11.6294(4) $\AA, b=14.3841(3) \AA, c=11.7731(4) \AA, \beta=107.965(3)^{\circ}, V=1873.38(10) \AA^{3}, Z=4, T=$ $99.96(16) \mathrm{K}, \mu(\mathrm{CuK} \alpha)=0.778 \mathrm{~mm}^{-1}$, Dcalc $=1.377 \mathrm{~g} / \mathrm{cm}^{3}, 13682$ reflections measured $\left(10.01^{\circ} \leq 2 \Theta \leq\right.$ $\left.139.874^{\circ}\right), 3455$ unique $\left(R_{\text {int }}=0.0355, \mathrm{R}_{\text {sigma }}=0.0279\right)$ which were used in all calculations. The final $R_{1}$ was $0.0338(\mathrm{I}>2 \sigma(\mathrm{I}))$ and $w R_{2}$ was 0.0882 (all data).

Details:

\section{Fixed Uiso}

At 1.2 times of: all $\mathrm{C}(\mathrm{H})$ groups, $\mathrm{All} \mathrm{C}(\mathrm{H}, \mathrm{H})$ groups

At 1.5 times of: all $\mathrm{C}(\mathrm{H}, \mathrm{H}, \mathrm{H})$ groups

2a. Secondary $\mathrm{CH}_{2}$ refined with riding coordinates: $\mathrm{C}_{5}\left(\mathrm{H}_{5 \mathrm{~A}}, \mathrm{H}_{5 \mathrm{~B}}\right), \mathrm{C}_{6}\left(\mathrm{H}_{6 \mathrm{~A}}, \mathrm{H}_{6 \mathrm{~B}}\right), \mathrm{C}_{8}\left(\mathrm{H}_{8 \mathrm{~A}}, \mathrm{H}_{8 \mathrm{~B}}\right), \mathrm{C}_{7}\left(\mathrm{H}_{7 \mathrm{~A}}, \mathrm{H}_{7 \mathrm{~B}}\right)$ 2b. Aromatic/amide $\mathrm{H}$ refined with riding coordinates: $\mathrm{C}_{20}\left(\mathrm{H}_{20}\right), \mathrm{C}_{11}\left(\mathrm{H}_{11}\right), \mathrm{C}_{17}\left(\mathrm{H}_{17}\right), \mathrm{C}_{21}\left(\mathrm{H}_{21}\right), \mathrm{C}_{14}\left(\mathrm{H}_{14}\right)$, $\mathrm{C}_{18}\left(\mathrm{H}_{18}\right), \mathrm{C}_{15}\left(\mathrm{H}_{15}\right), \mathrm{C}_{13}\left(\mathrm{H}_{13}\right), \mathrm{C}_{12}\left(\mathrm{H}_{12}\right)$

2c. Idealised Me refined as rotating group: $\mathrm{C}_{23}\left(\mathrm{H}_{23 \mathrm{~A}}, \mathrm{H}_{23 \mathrm{~B}}, \mathrm{H}_{23 \mathrm{C}}\right)$ 
3-(Quinolin-2-yl)isoxazol-5(4H)-one/3-(quinolin-2-yl)isoxazol-5-ol 11b, ${ }^{1} \mathrm{H}$ NMR, $400 \mathrm{MHz}$, DMSO- $d_{6}$
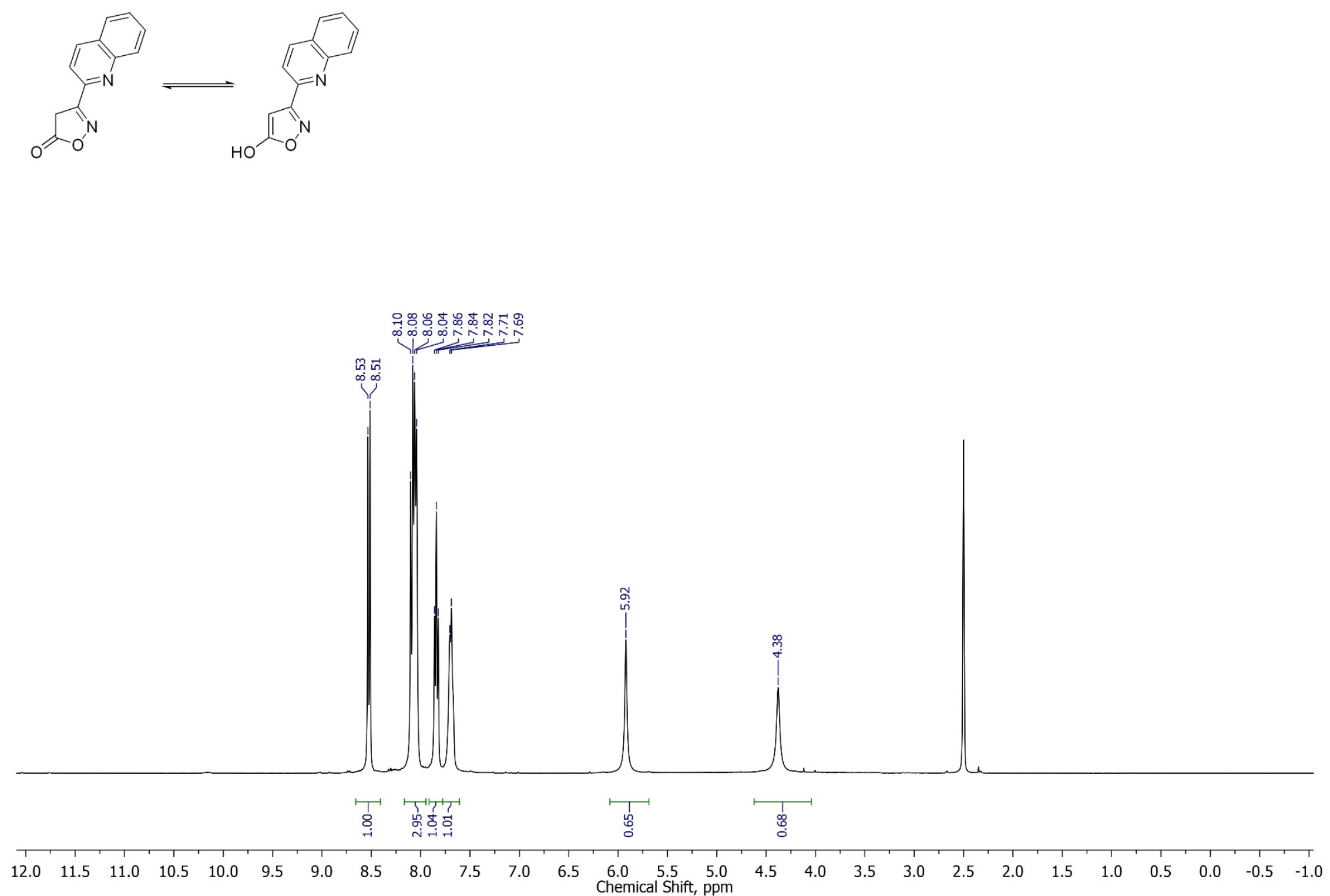
4-(4-Chlorobenzylidene)-3-(pyridin-2-yl)isoxazol-5(4H)-one $1 \mathrm{e},{ }^{1} \mathrm{H} \mathrm{NMR}, 400 \mathrm{MHz}, \mathrm{CDCl}_{3}$
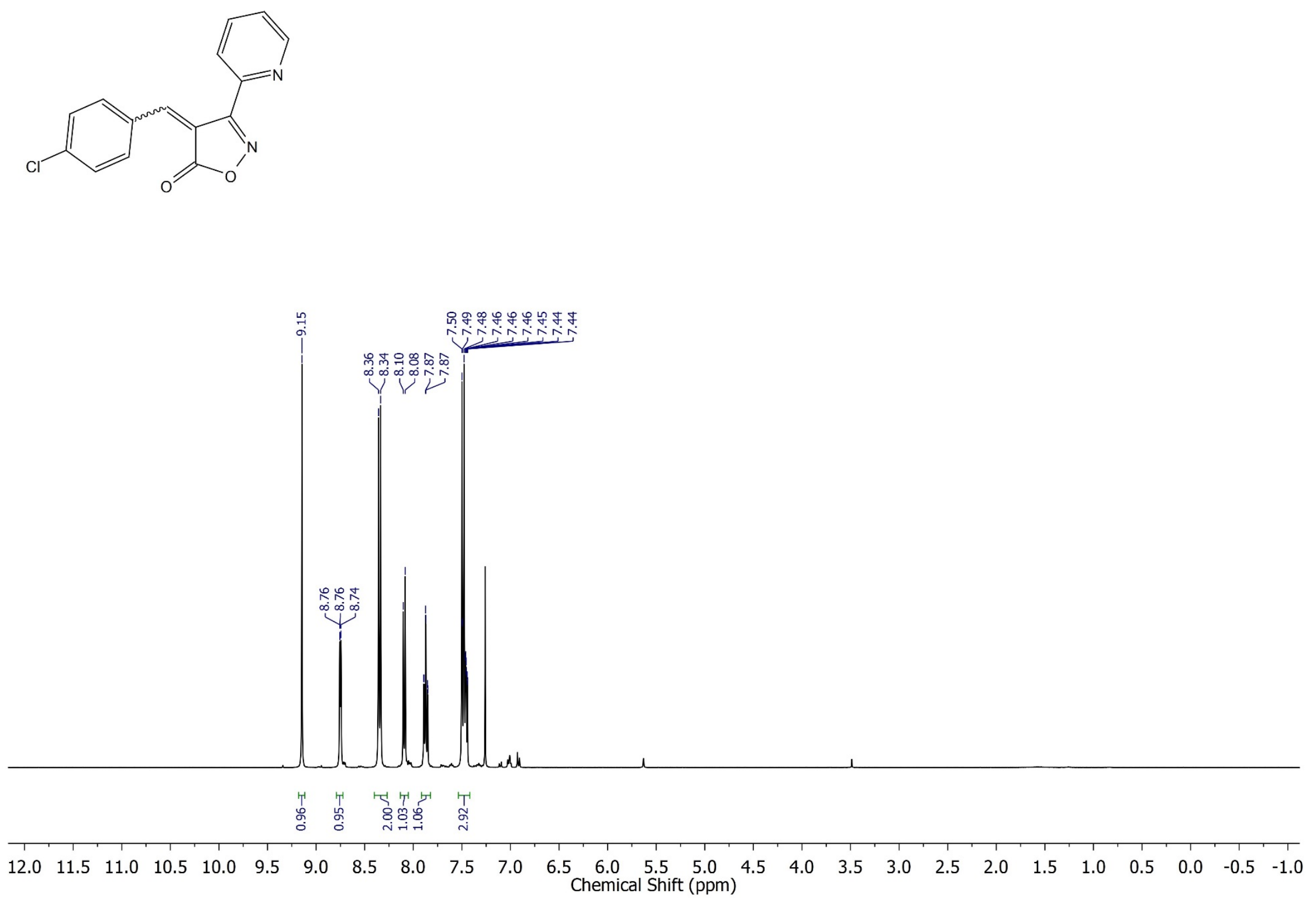
4-(4-Chlorobenzylidene)-3-(pyridin-2-yl)isoxazol-5(4H)-one $1 \mathrm{e},{ }^{13} \mathrm{C}\left\{{ }^{1} \mathrm{H}\right\} \mathrm{NMR}, 100 \mathrm{MHz}, \mathrm{CDCl}_{3}$

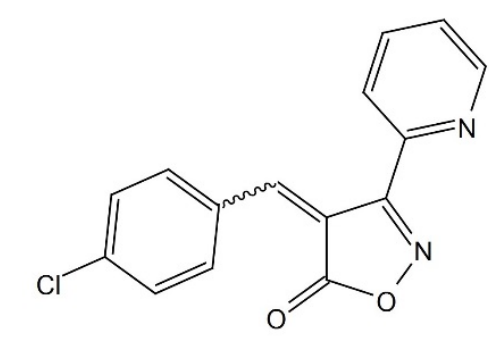

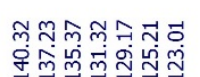
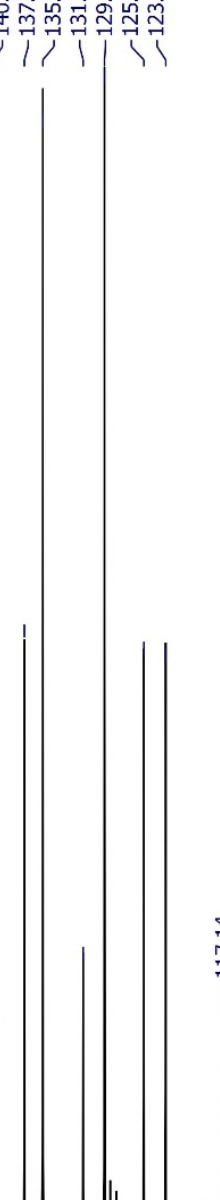

$$
210
$$

200190 
4-(4-Chlorobenzylidene)-3-(pyridin-2-yl)isoxazol-5(4H)-one 1e, DEPT, $100 \mathrm{MHz}^{-\mathrm{CDCl}_{3}}$
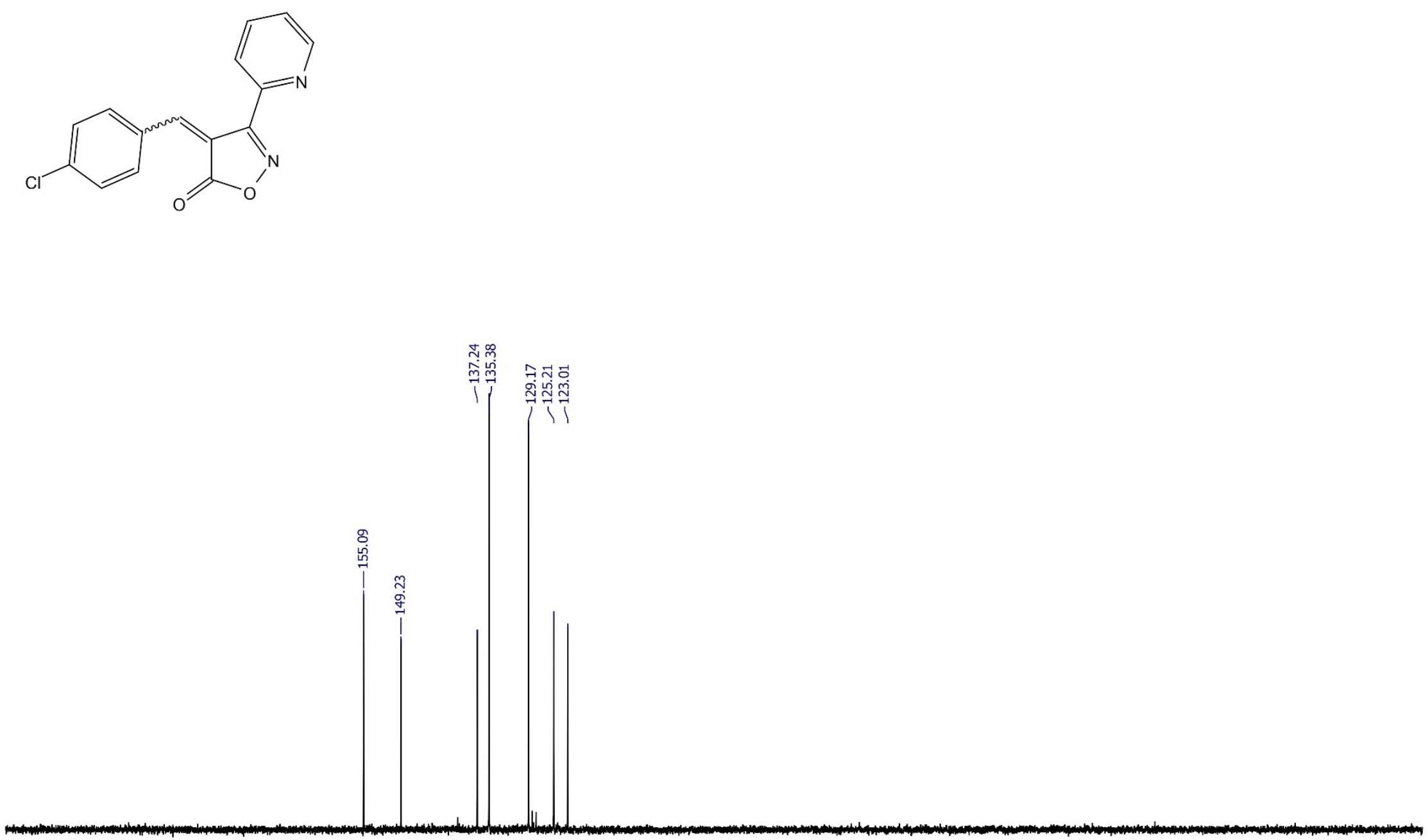

1

200 
4-(4-Methylbenzylidene)-3-(quinolin-2-yl)isoxazol-5(4H)-one 1f, ${ }^{1} \mathrm{H} \mathrm{NMR}, 400 \mathrm{MHz}, \mathrm{CDCl}_{3}$
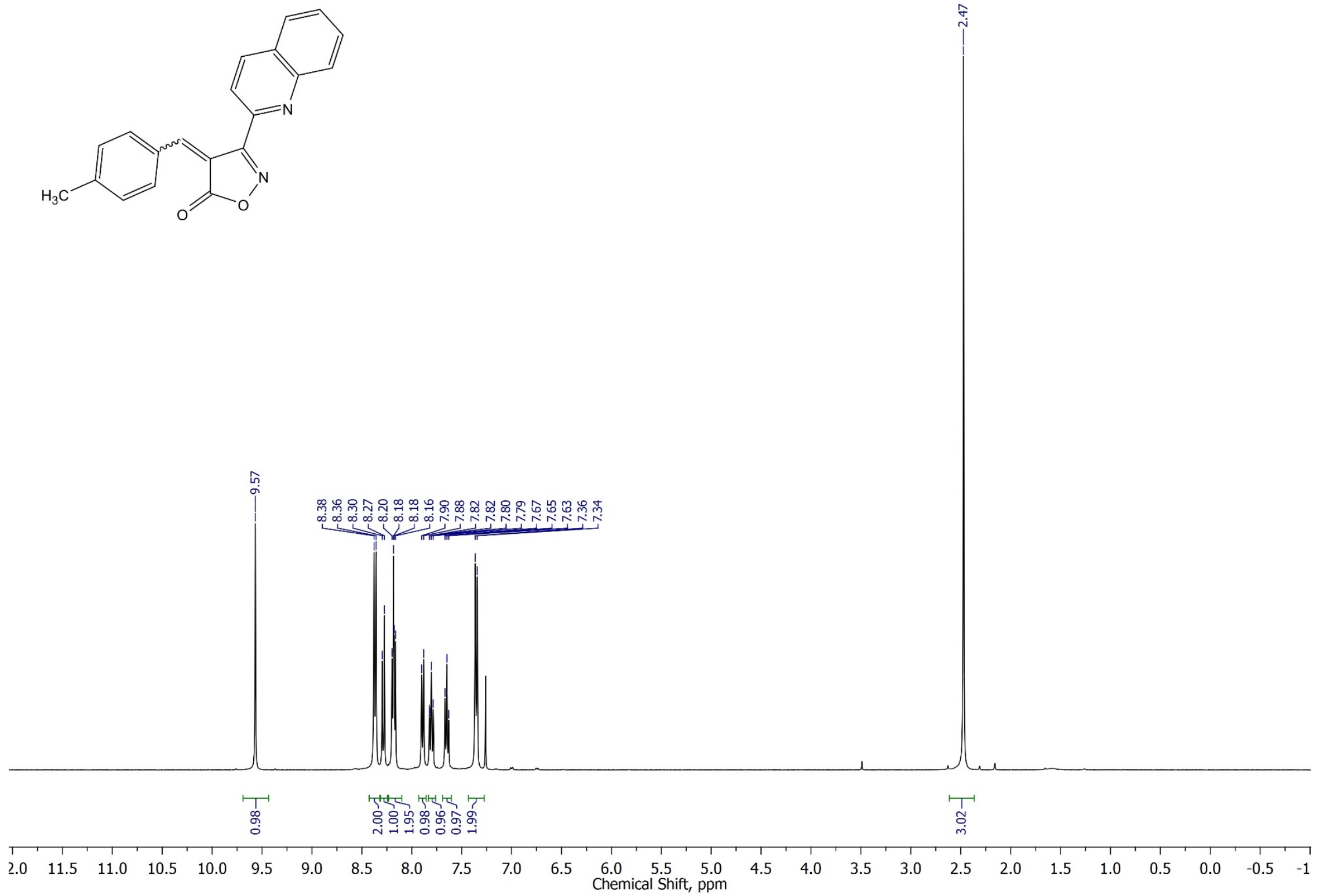
4-(4-Methylbenzylidene)-3-(quinolin-2-yl)isoxazol-5(4H)-one 1f, ${ }^{13} \mathrm{C}\left\{{ }^{1} \mathrm{H}\right\} \mathrm{NMR}, 100 \mathrm{MHz}, \mathrm{CDCl}_{3}$
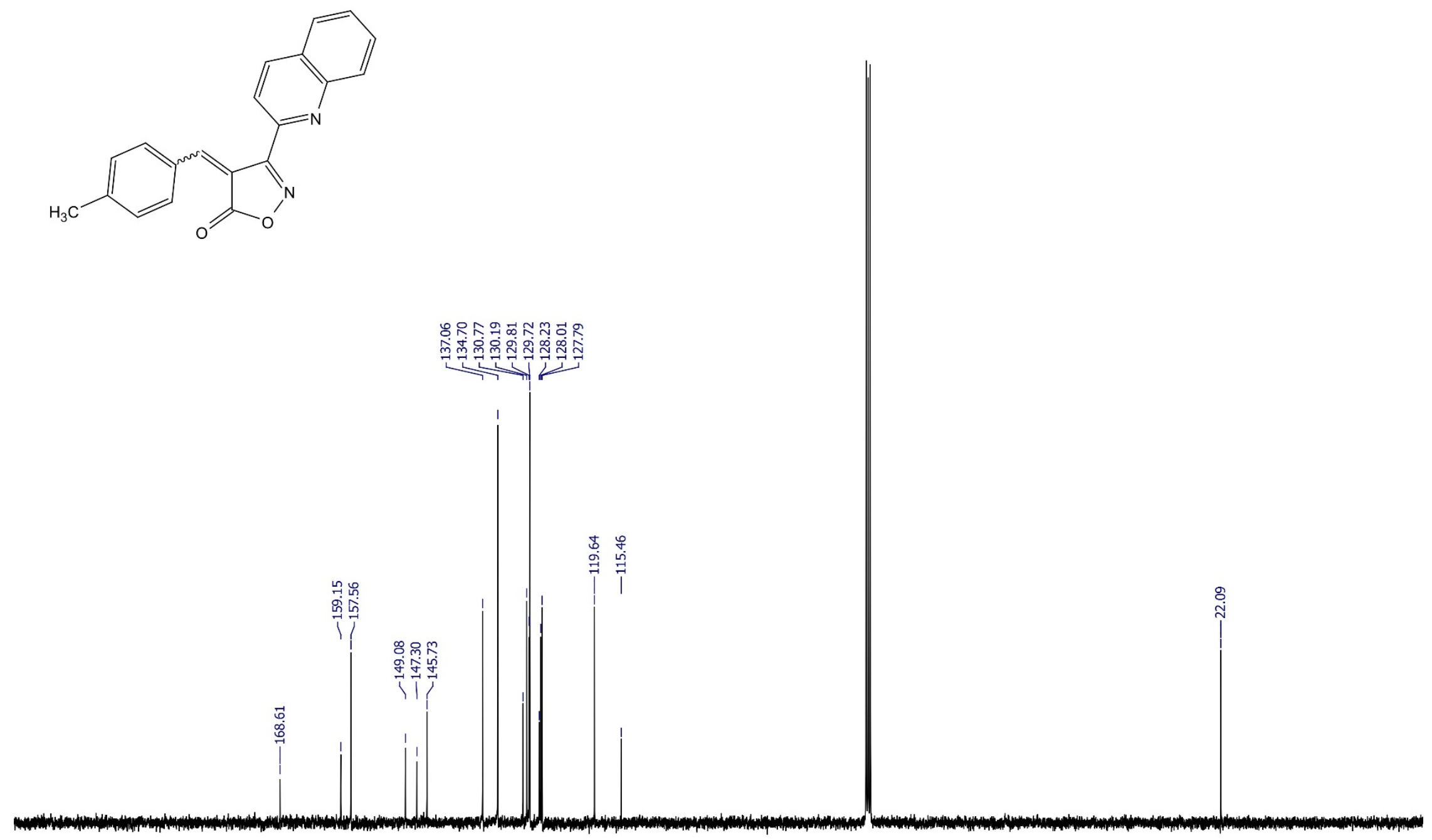

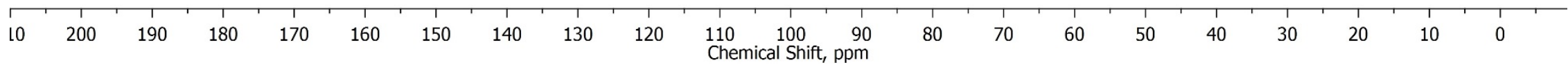




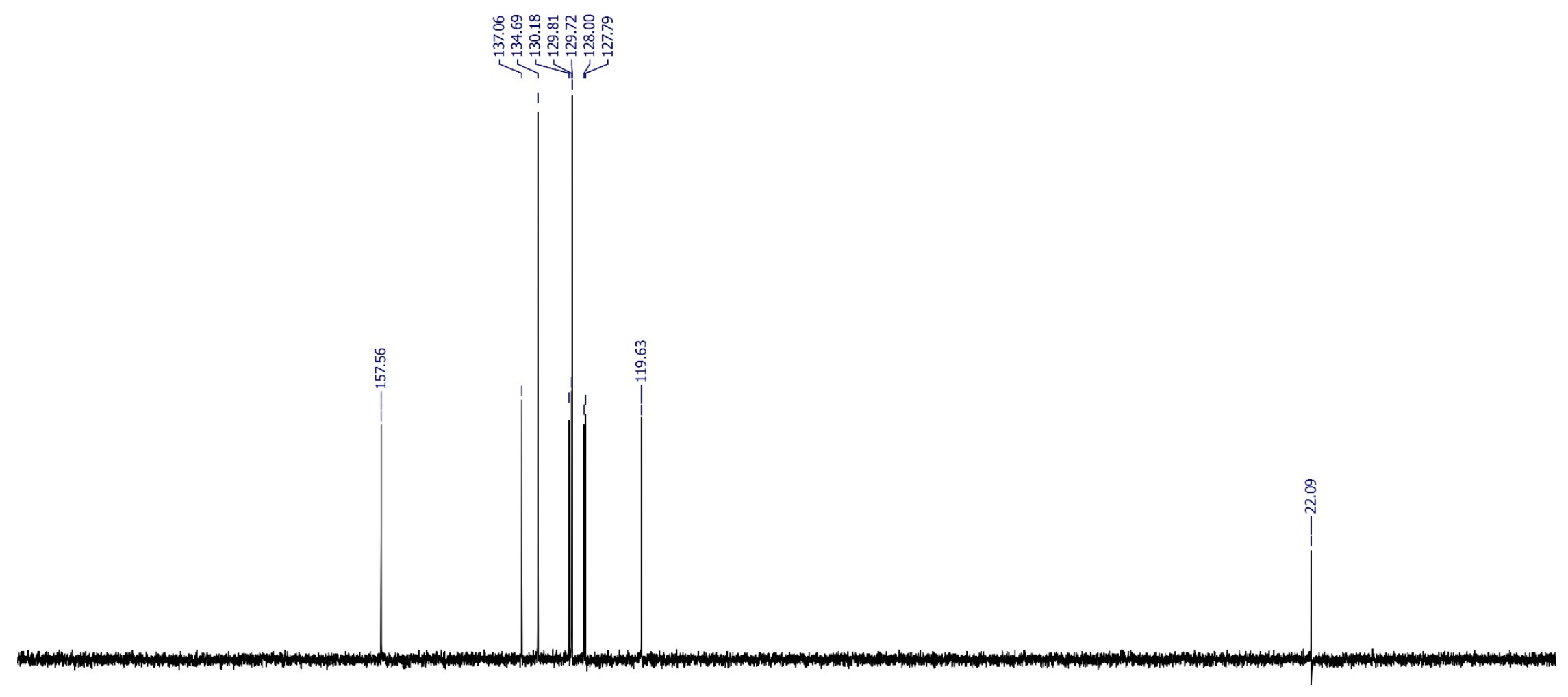

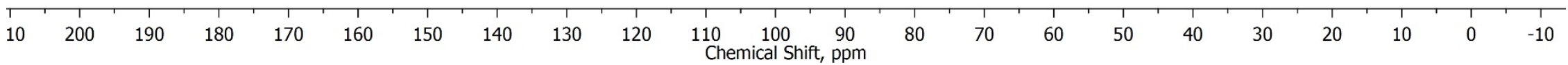


3-(4-Bromophenyl)-4-(cyclopropylmethylene)isoxazol-5(4H)-one 1i, ${ }^{1} \mathrm{H} \mathrm{NMR}, 400 \mathrm{MHz}, \mathrm{CDCl}_{3}$

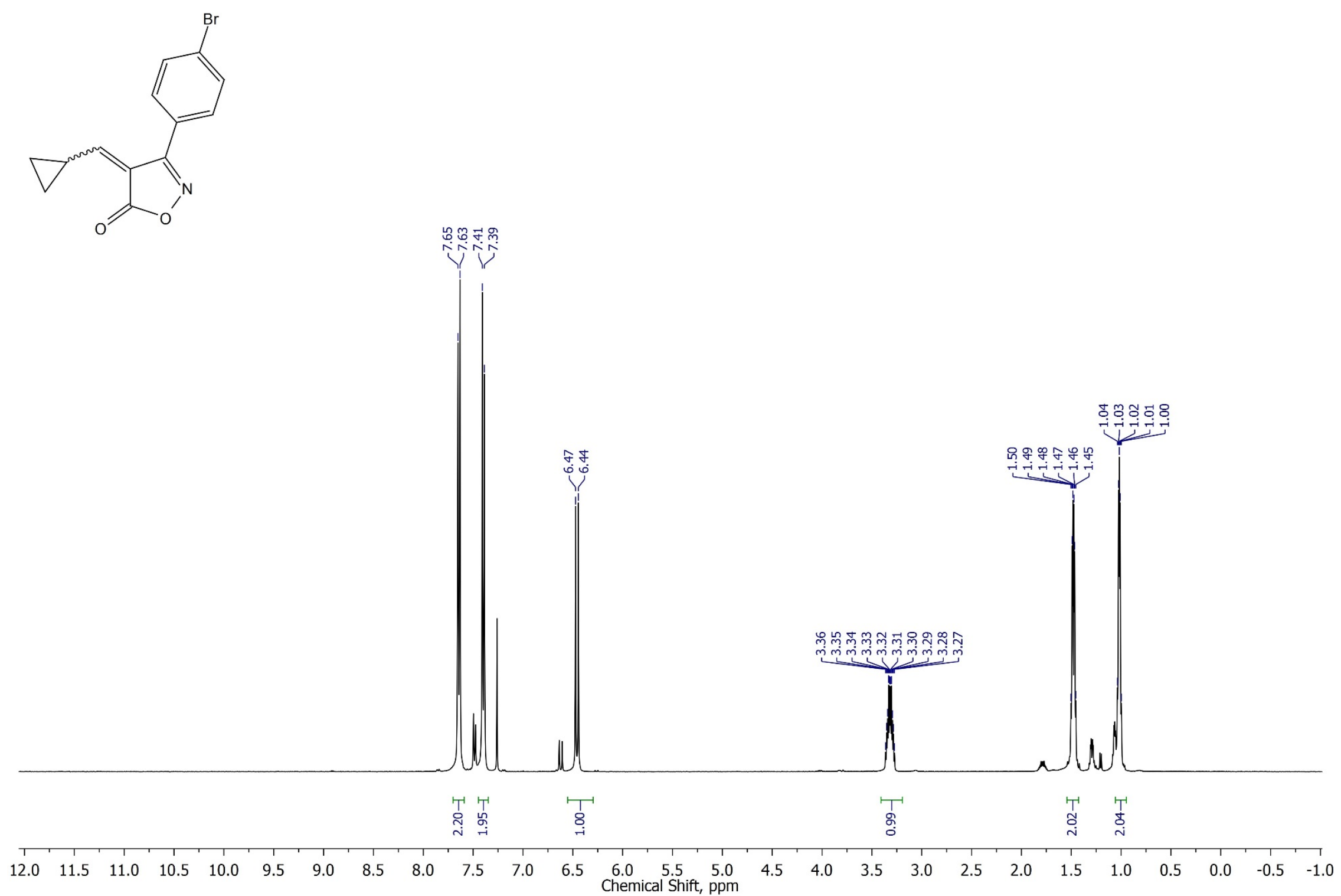


3-(4-Bromophenyl)-4-(cyclopropylmethylene)isoxazol-5(4H)-one 1i, ${ }^{13} \mathrm{C}\left\{{ }^{1} \mathrm{H}\right\} \mathrm{NMR}, 100 \mathrm{MHz}, \mathrm{CDCl}_{3}$
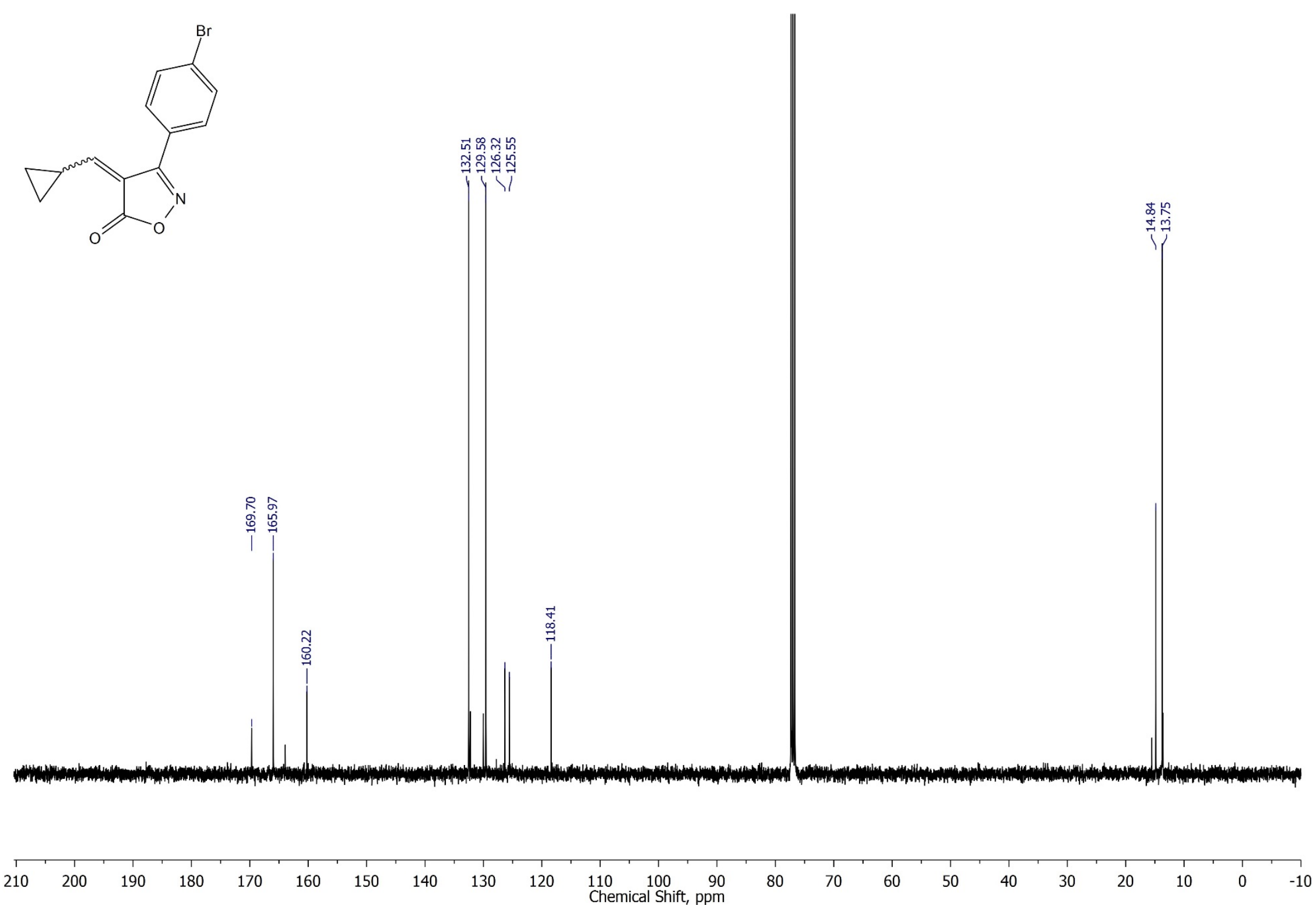
3-(4-Bromophenyl)-4-(cyclopropylmethylene)isoxazol-5(4H)-one 1i, DEPT, $100 \mathrm{MHz}, \mathrm{CDCl}_{3}$
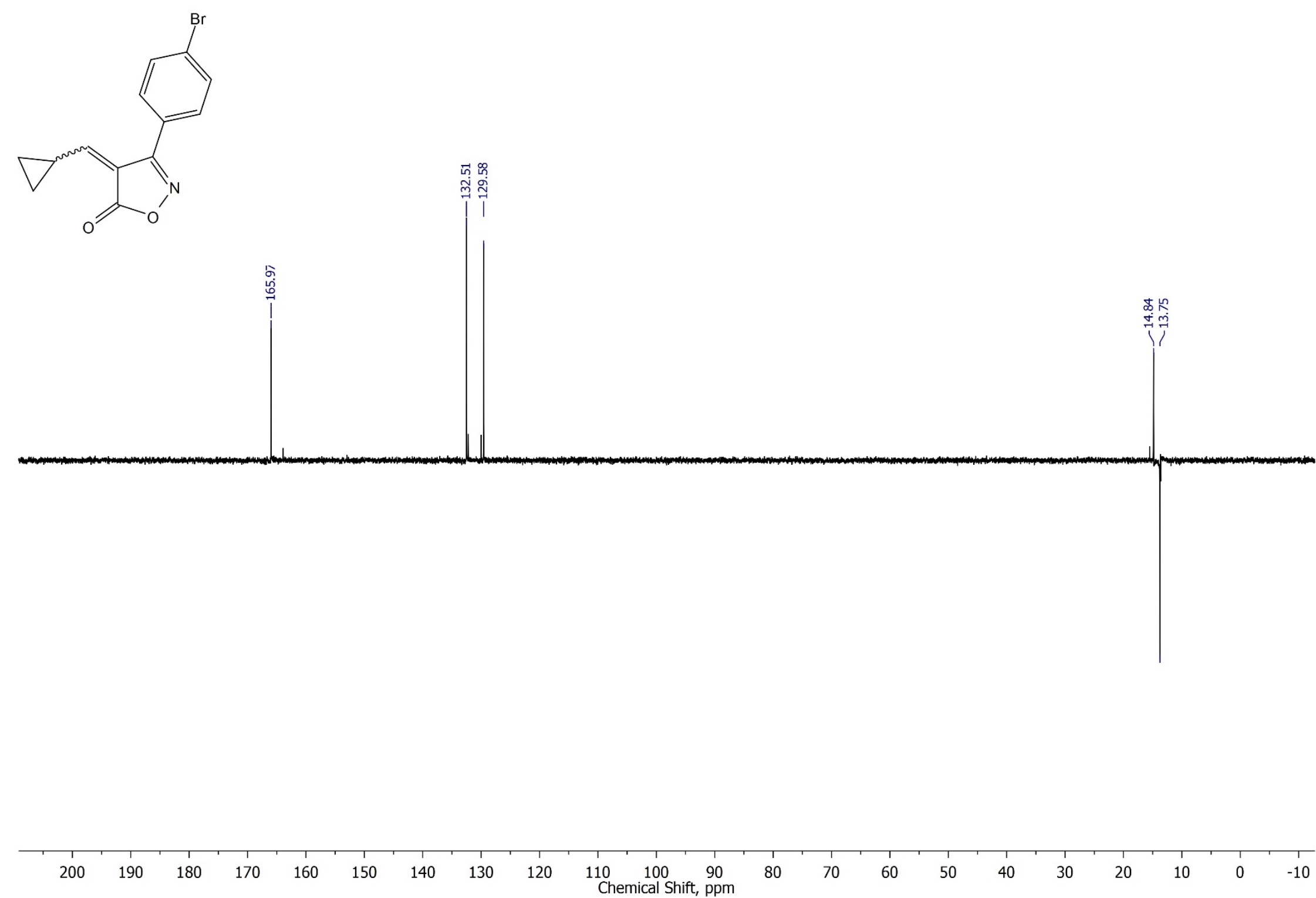
3-(tert-Butyl)-4-(4-(tert-butyl)benzylidene)isoxazol-5(4H)-one 1j, ${ }^{1} \mathrm{H} \mathrm{NMR}, 400 \mathrm{MHz}, \mathrm{CDCl}_{3}$
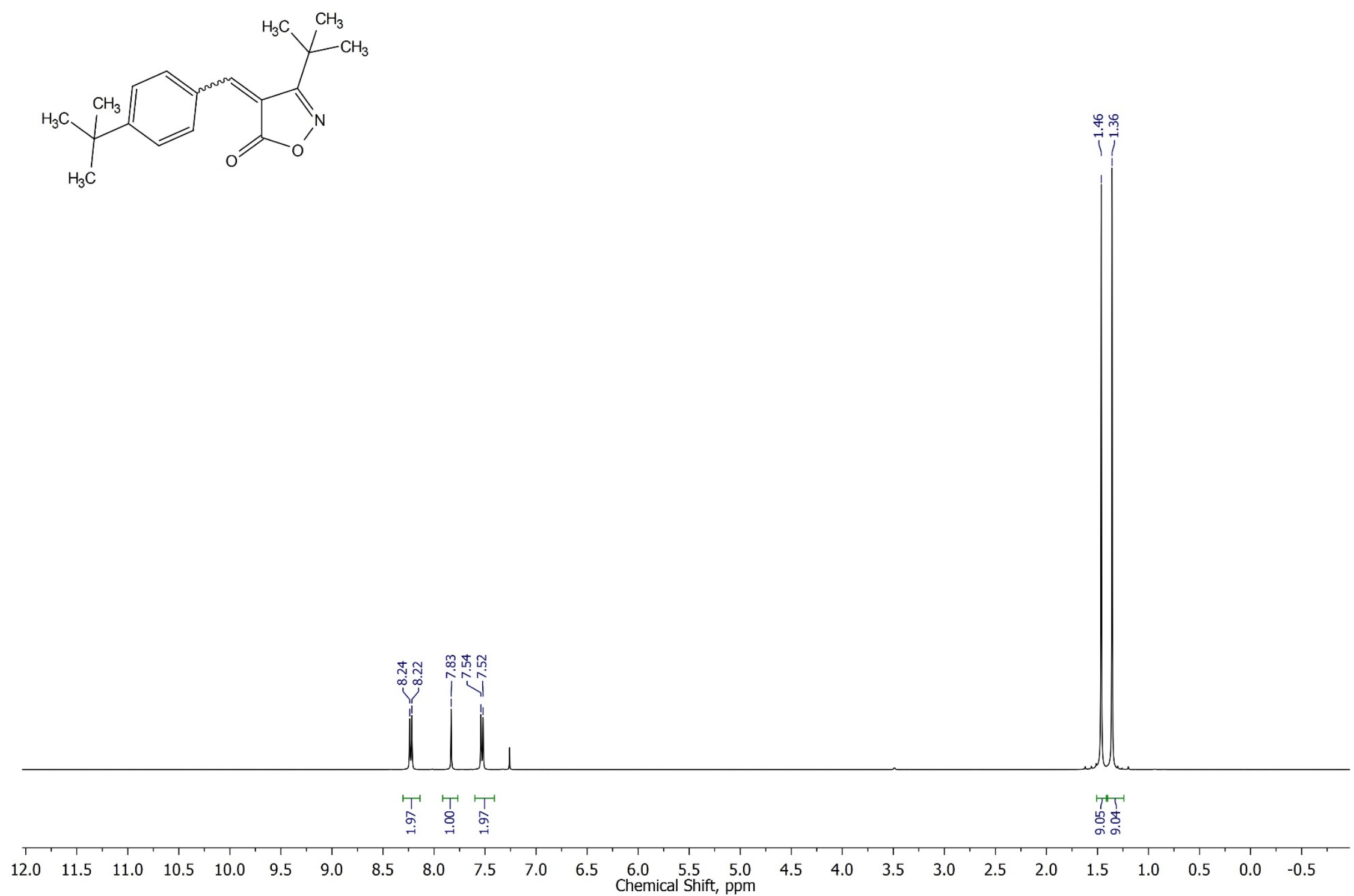
3-(tert-Butyl)-4-(4-(tert-butyl)benzylidene)isoxazol-5(4H)-one $1 \mathrm{j},{ }^{13} \mathrm{C}\left\{{ }^{1} \mathrm{H}\right\} \mathrm{NMR}, 100 \mathrm{MHz}, \mathrm{CDCl}_{3}$
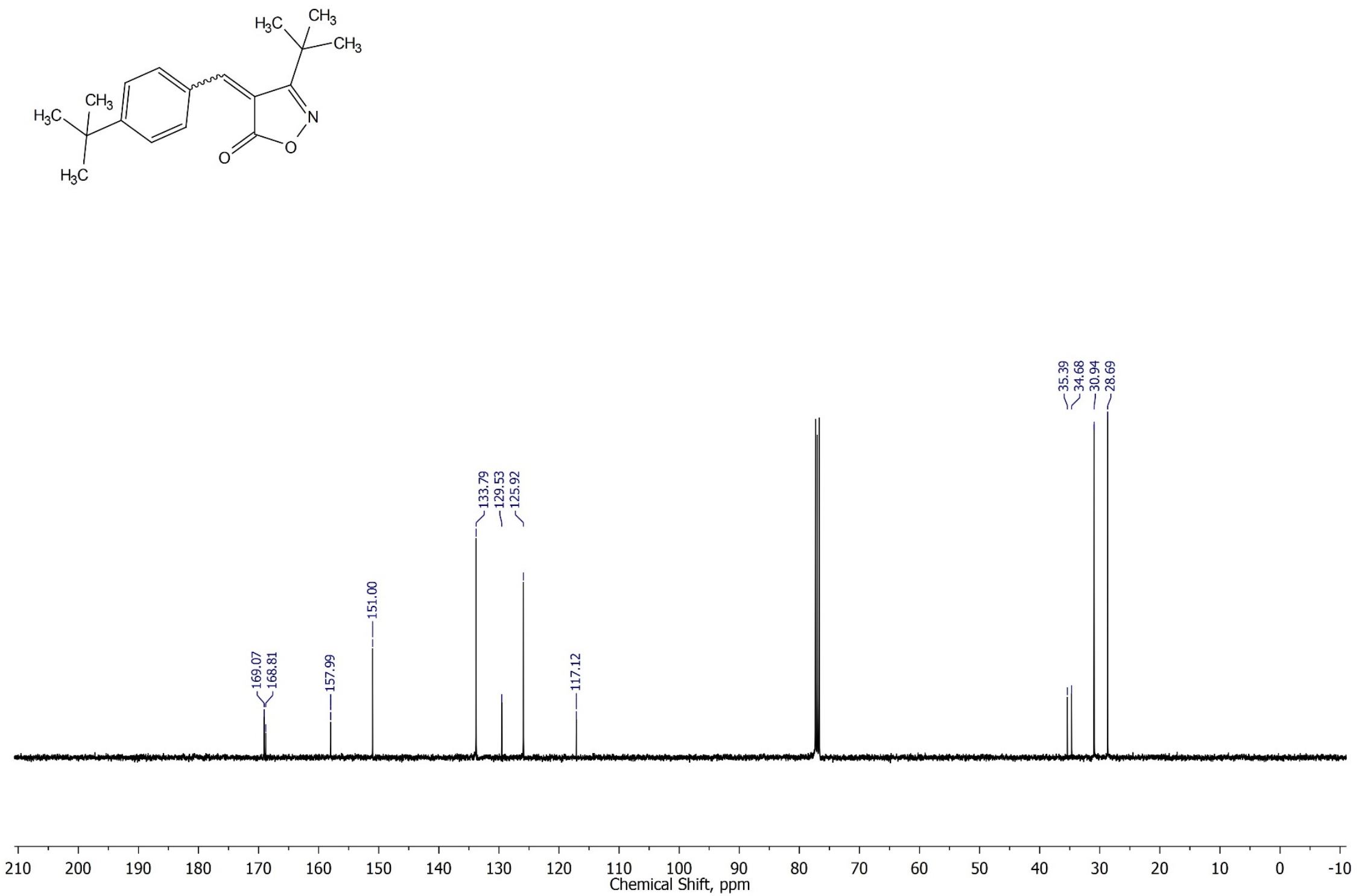

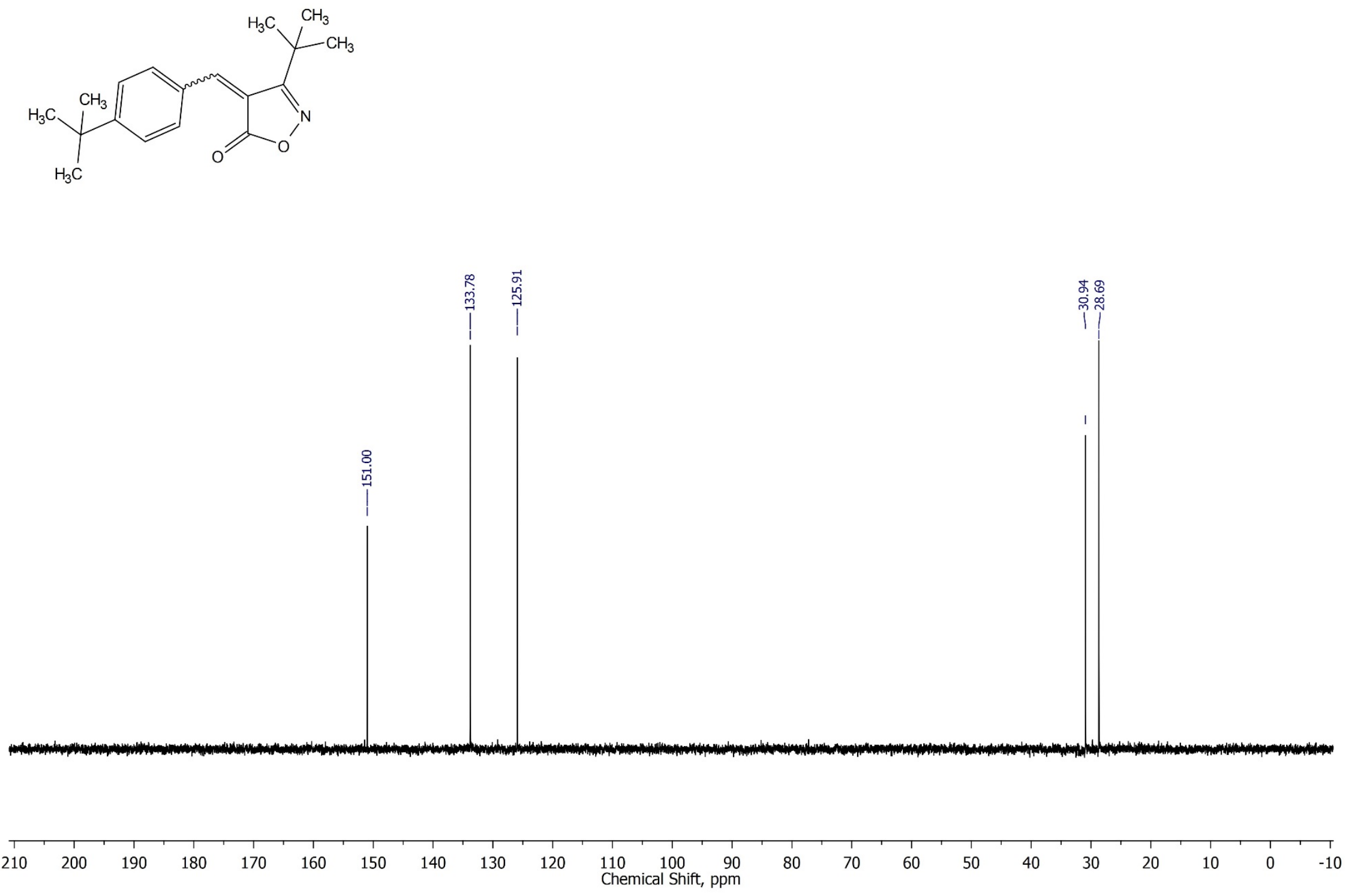
4-(7-Methoxy-3,4-dihydronaphthalen-1-yl)morpholine 2d, ${ }^{1} \mathrm{H} \mathrm{NMR}, 400 \mathrm{MHz}, \mathrm{CDCl}_{3}$

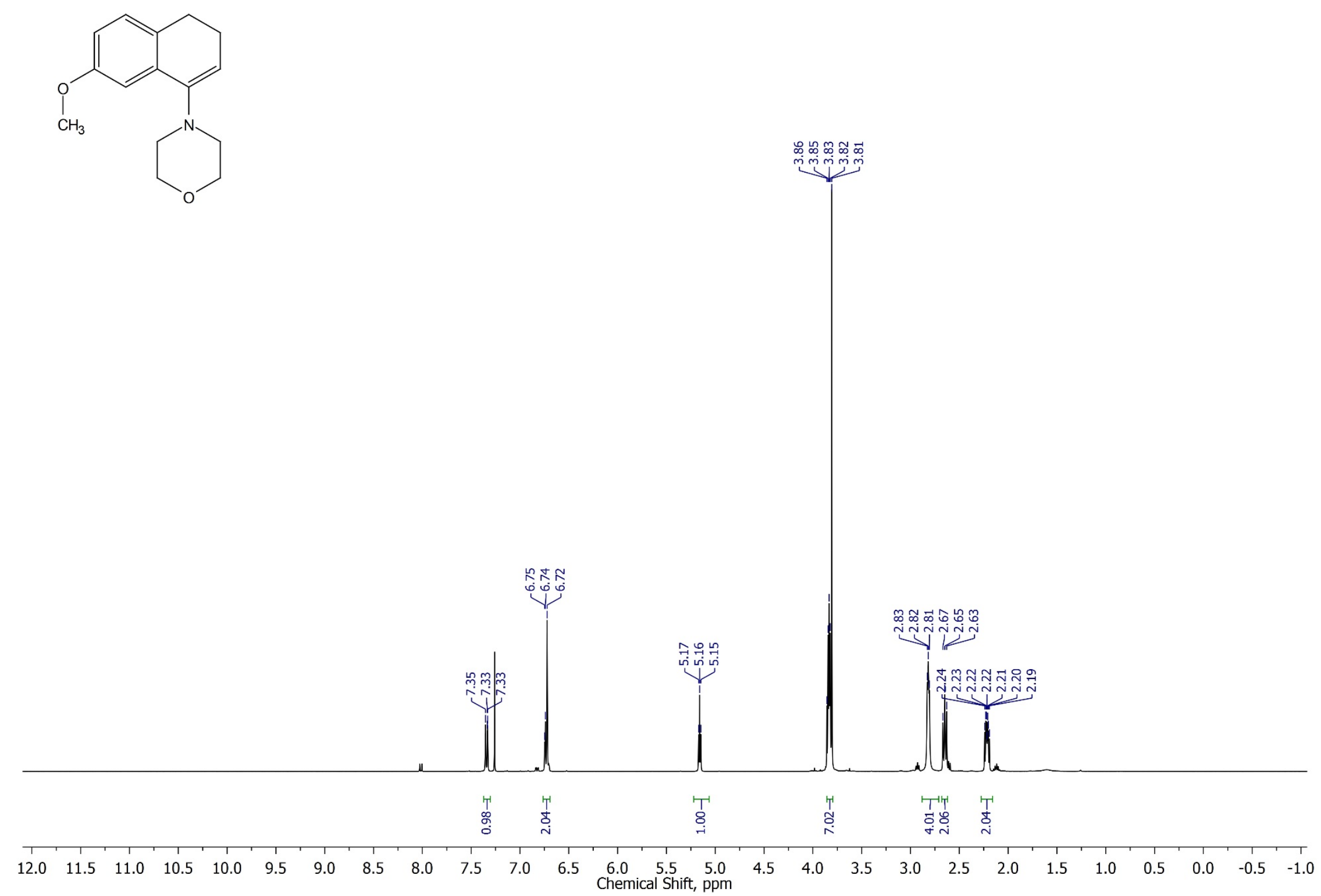


4-(7-Methoxy-3,4-dihydronaphthalen-1-yl)morpholine 2d, ${ }^{13} \mathrm{C}\left\{{ }^{1} \mathrm{H}\right\}$ NMR, $100 \mathrm{MHz}, \mathrm{CDCl}_{3}$
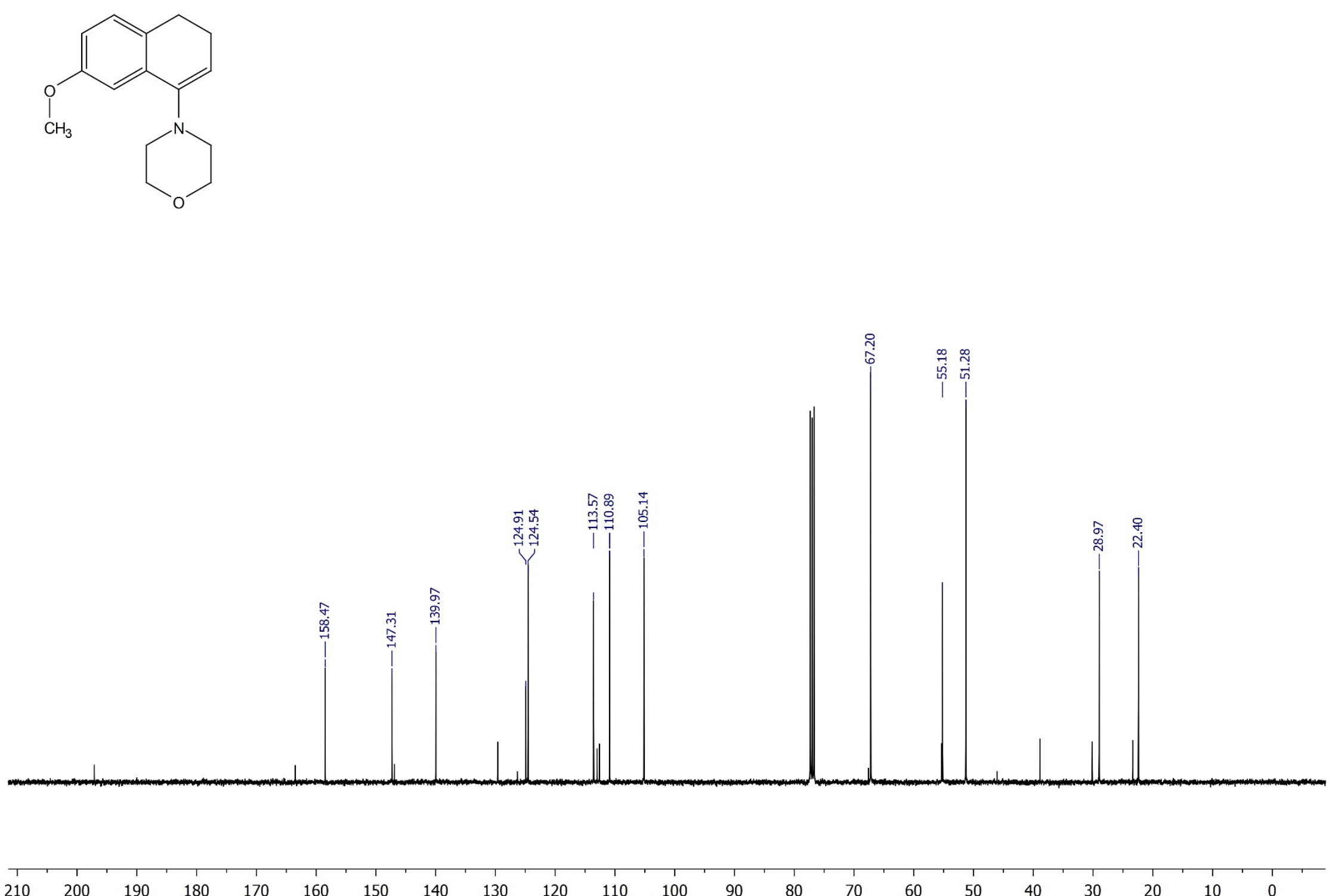

$180 \quad 17$

160

$150 \quad 140$

$130 \quad 120$

$\begin{array}{lll}110 & 100 \quad 90\end{array}$

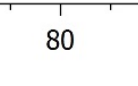

$60 \quad 50$

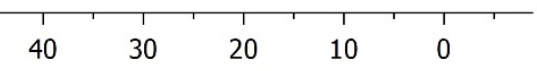


4-(7-Methoxy-3,4-dihydronaphthalen-1-yl)morpholine 2d, DEPT, $100 \mathrm{MHz}, \mathrm{CDCl}_{3}$
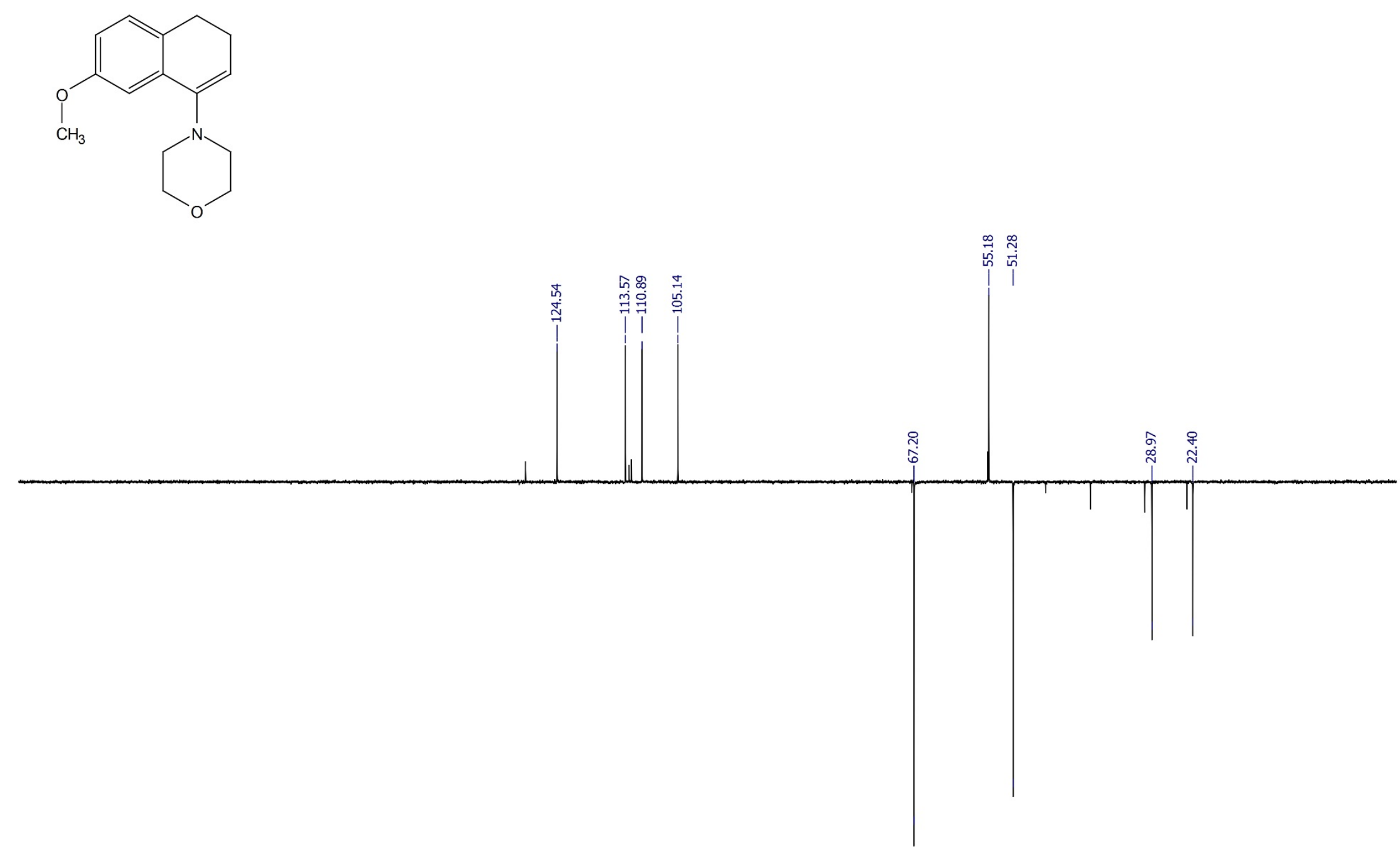

$$
210
$$

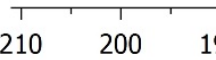

$190 \quad 180$

170 

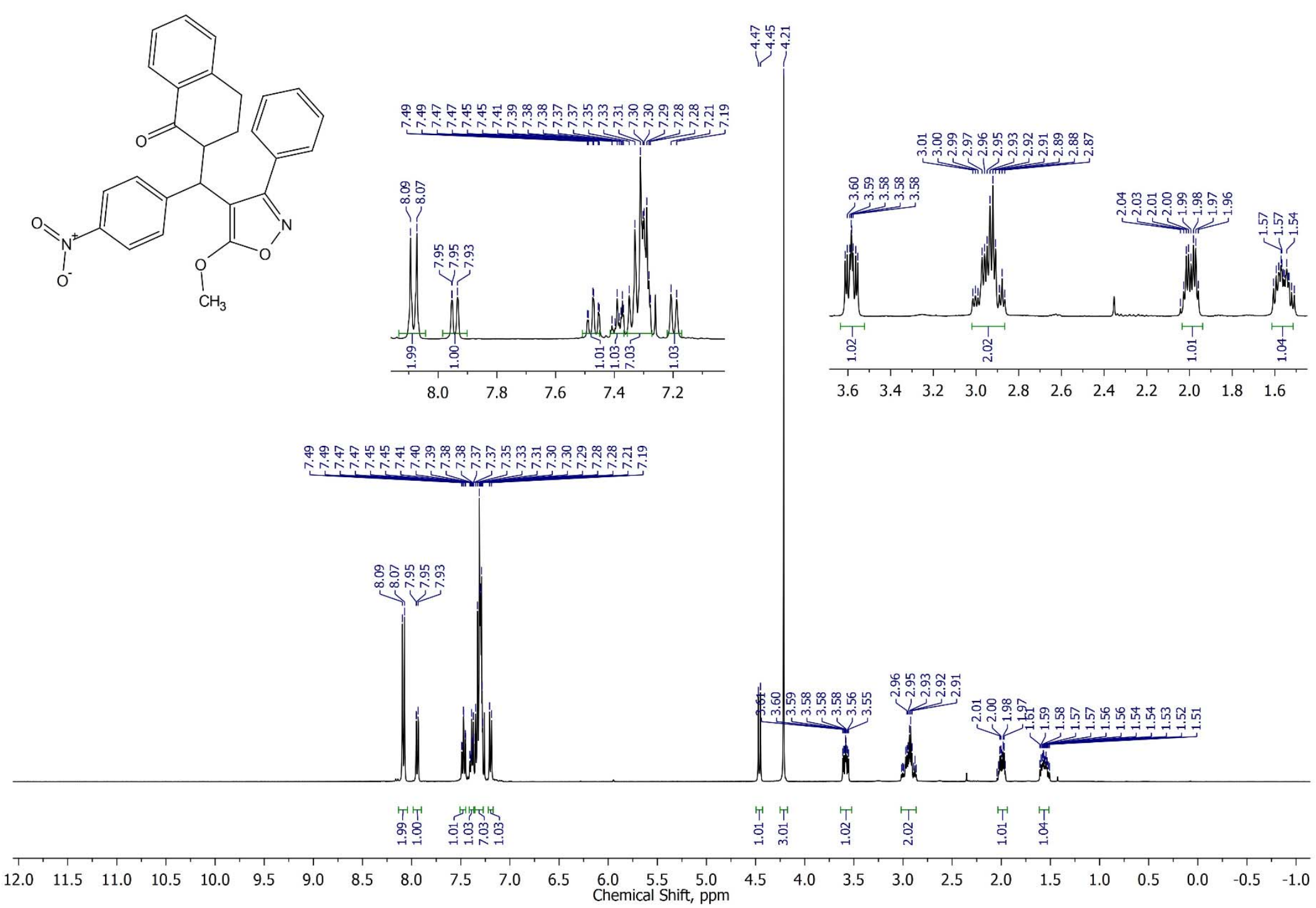

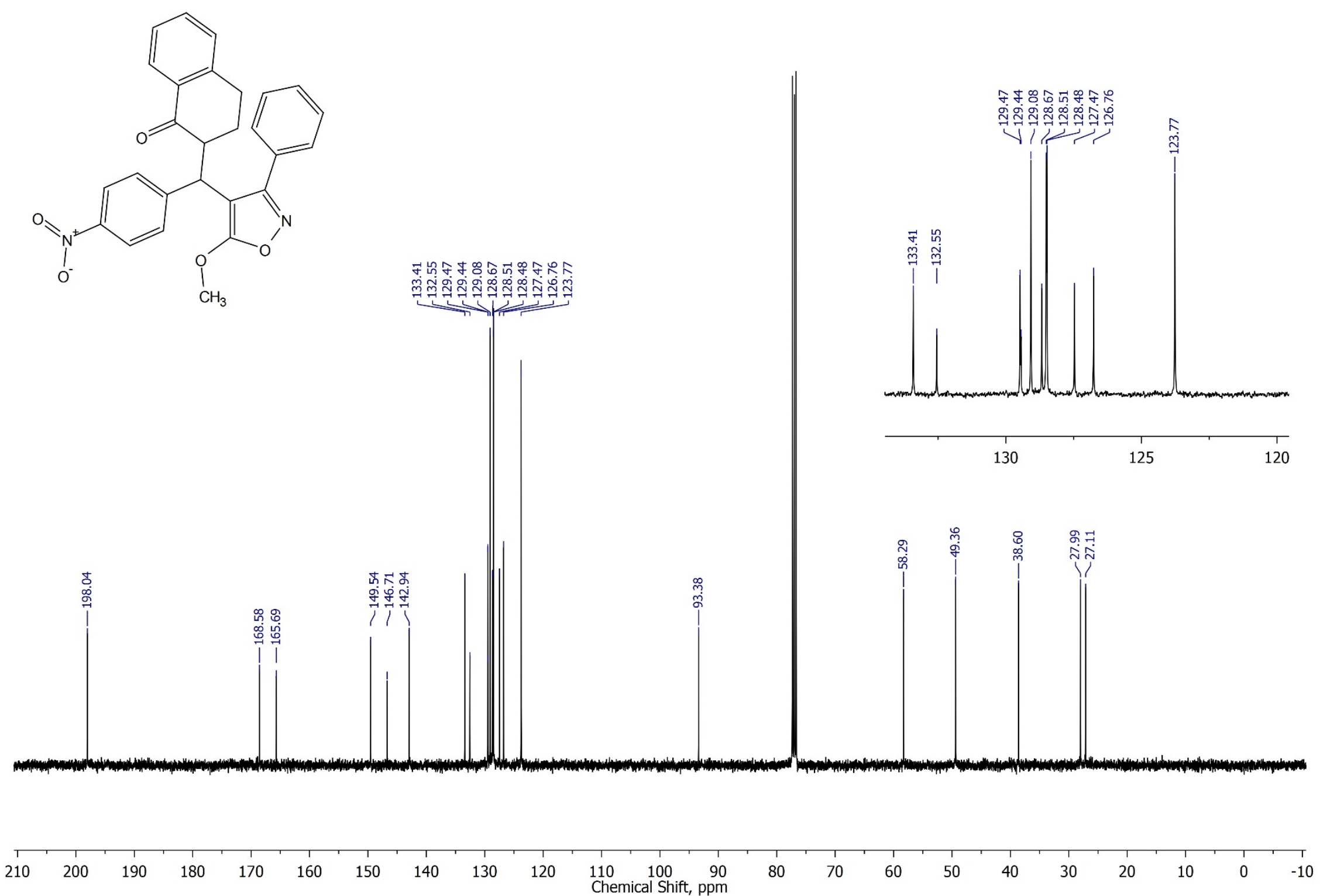
2-((5-Methoxy-3-phenylisoxazol-4-yl)(4-nitrophenyl)methyl)-3,4-dihydronaphthalen-1(2H)-one 4i, DEPT, $100 \mathrm{MHz}^{\mathrm{C}} \mathrm{CDCl}_{3}$

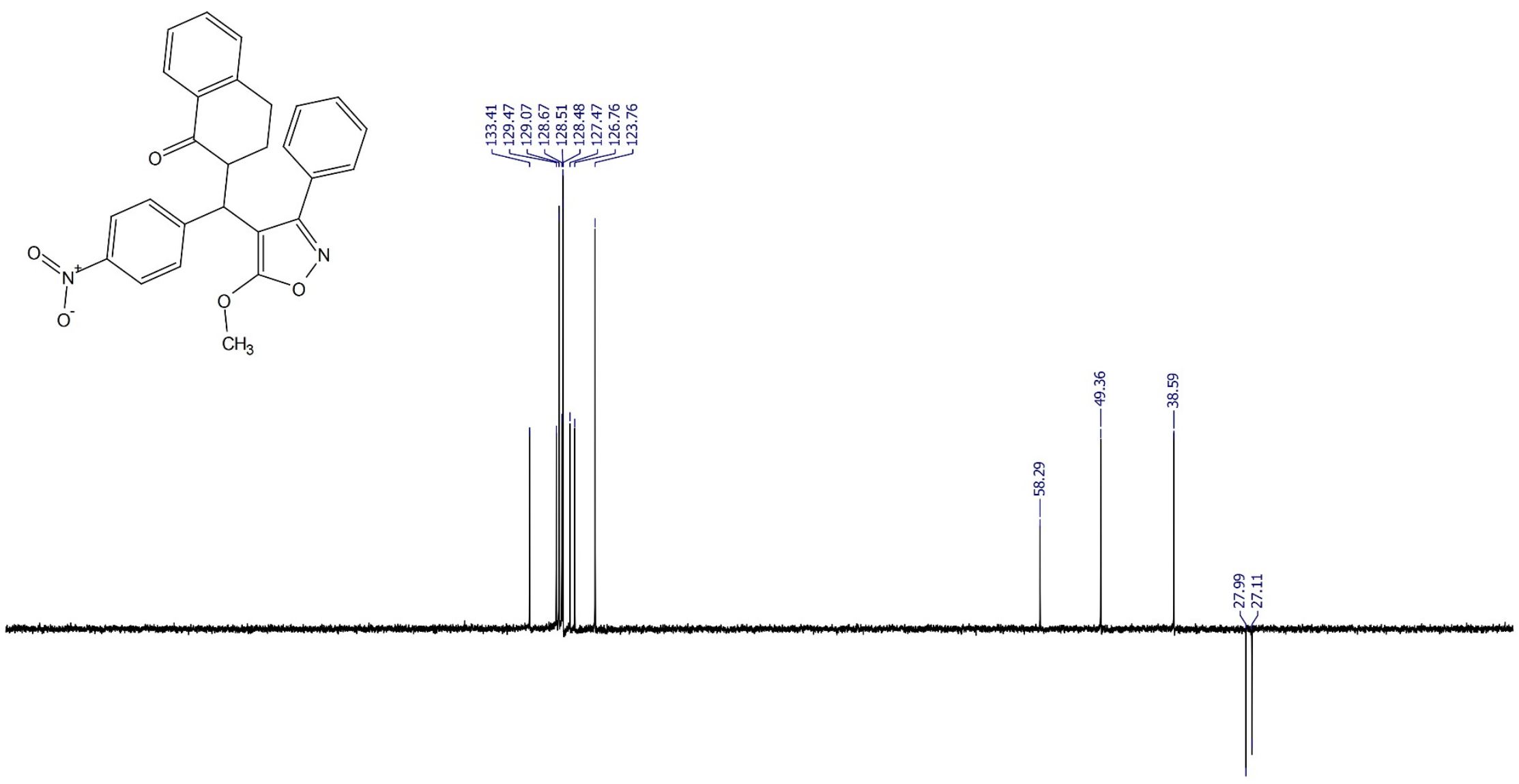

$\begin{array}{llllllllllllllllllllll}210 & 200 & 190 & 180 & 170 & 160 & 150 & 140 & 130 & 120 & \begin{array}{c}110 \\ \text { Chemical Shift, ppm }\end{array} & 80 & 70 & 60 & 50 & 40 & 30 & 20 & 10 & 0 & -10\end{array}$ 
2-Methyl-4-((4-nitrophenyl)(1-oxo-1,2,3,4-tetrahydronaphthalen-2-yl)methyl)-3-phenylisoxazol-5(2H)-one 4'i, ${ }^{1} \mathrm{H}^{\mathrm{NMR}}, 400 \mathrm{MHz}, \mathrm{CDCl}_{3}$

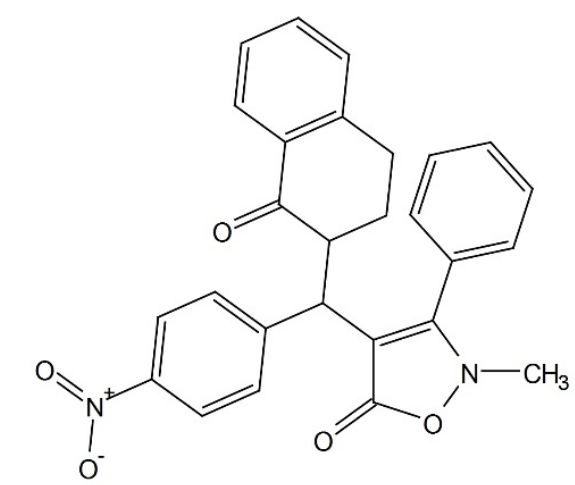

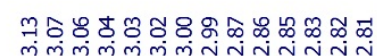

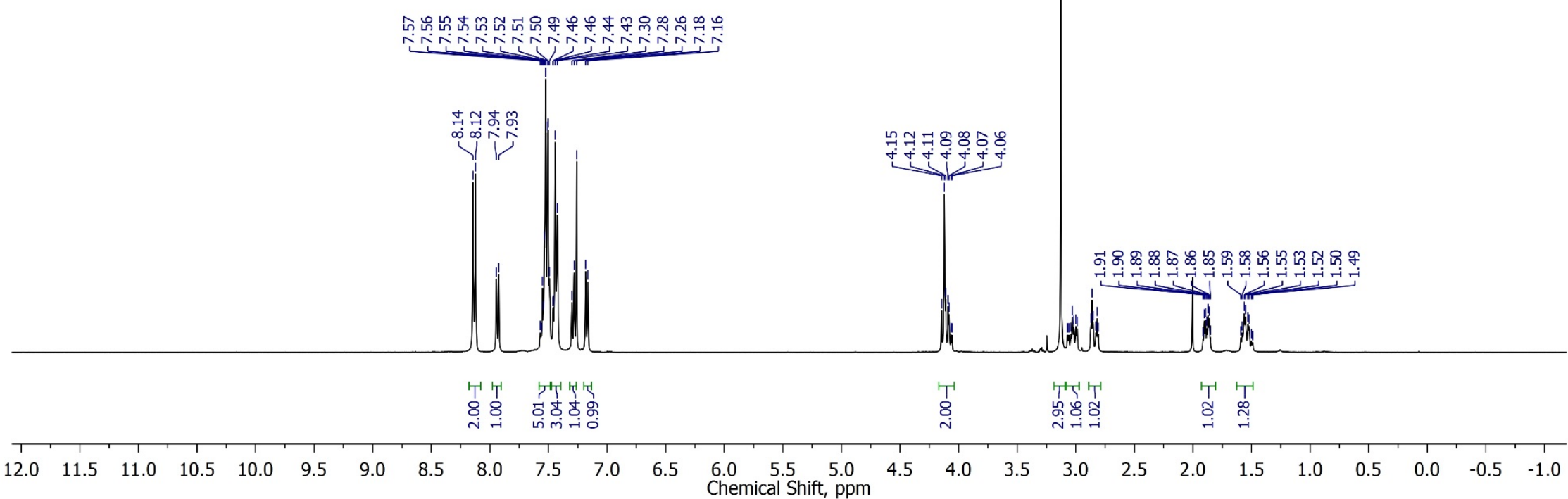


2-Methyl-4-((4-nitrophenyl)(1-oxo-1,2,3,4-tetrahydronaphthalen-2-yl)methyl)-3-phenylisoxazol-5(2H)-one 4'i, ${ }^{13} \mathrm{C}\left\{{ }^{1} \mathrm{H}\right\}$ NMR, $100 \mathrm{MHz}, \mathrm{CDCl}{ }_{3}$
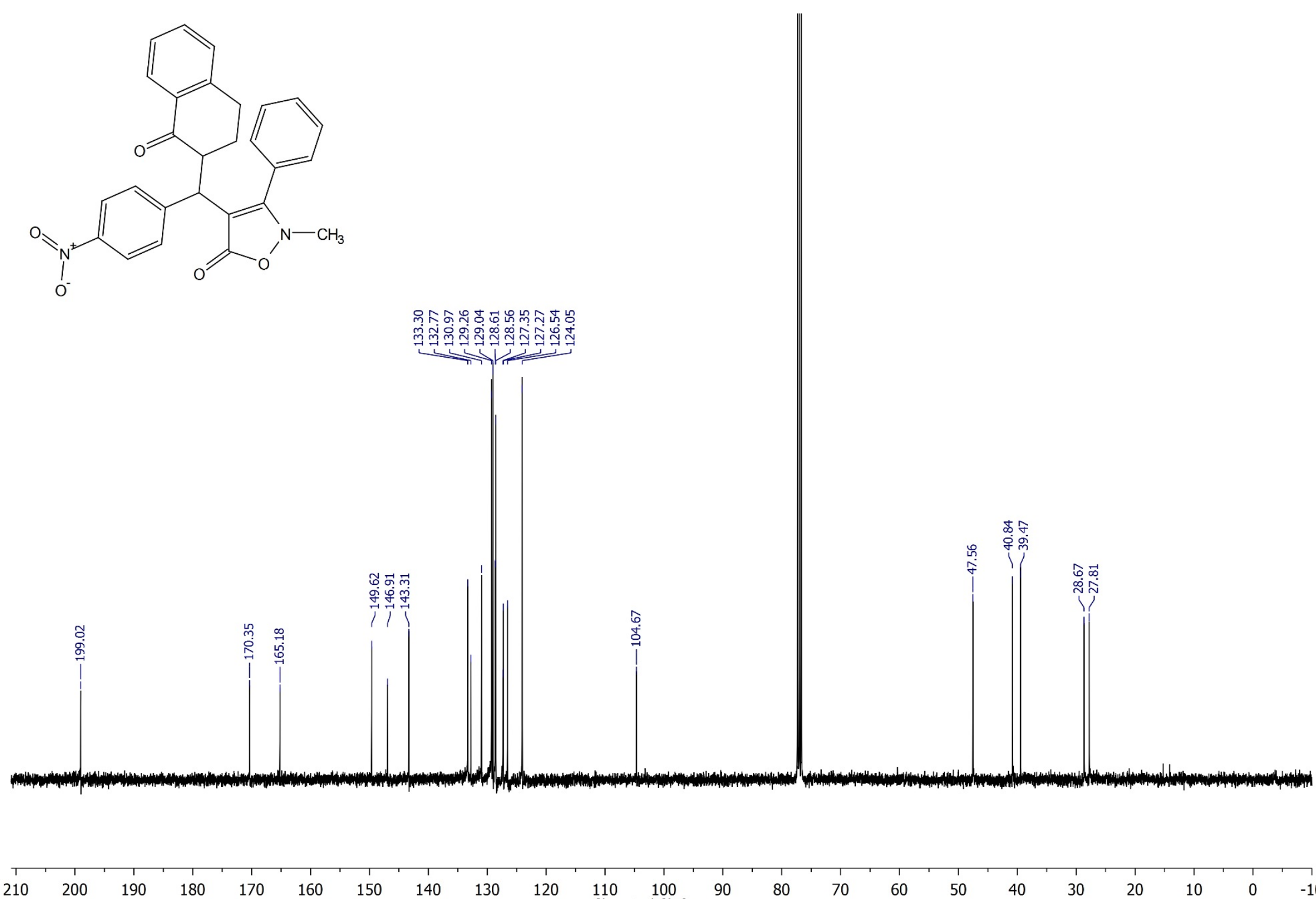

180

$170 \quad 160$

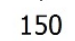

140

130

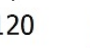

$\begin{array}{lrr}110 & 100 & 90 \\ \text { Chemical Shift, } & \text { ppm }\end{array}$

$80 \quad 70$

60

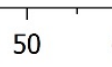

40

30

$10 \quad 0 \quad-10$ 
2-Methyl-4-((4-nitrophenyl)(1-oxo-1,2,3,4-tetrahydronaphthalen-2-yl)methyl)-3-phenylisoxazol-5(2H)-one 4'i, DEPT, 100 MHz, CDCl 3

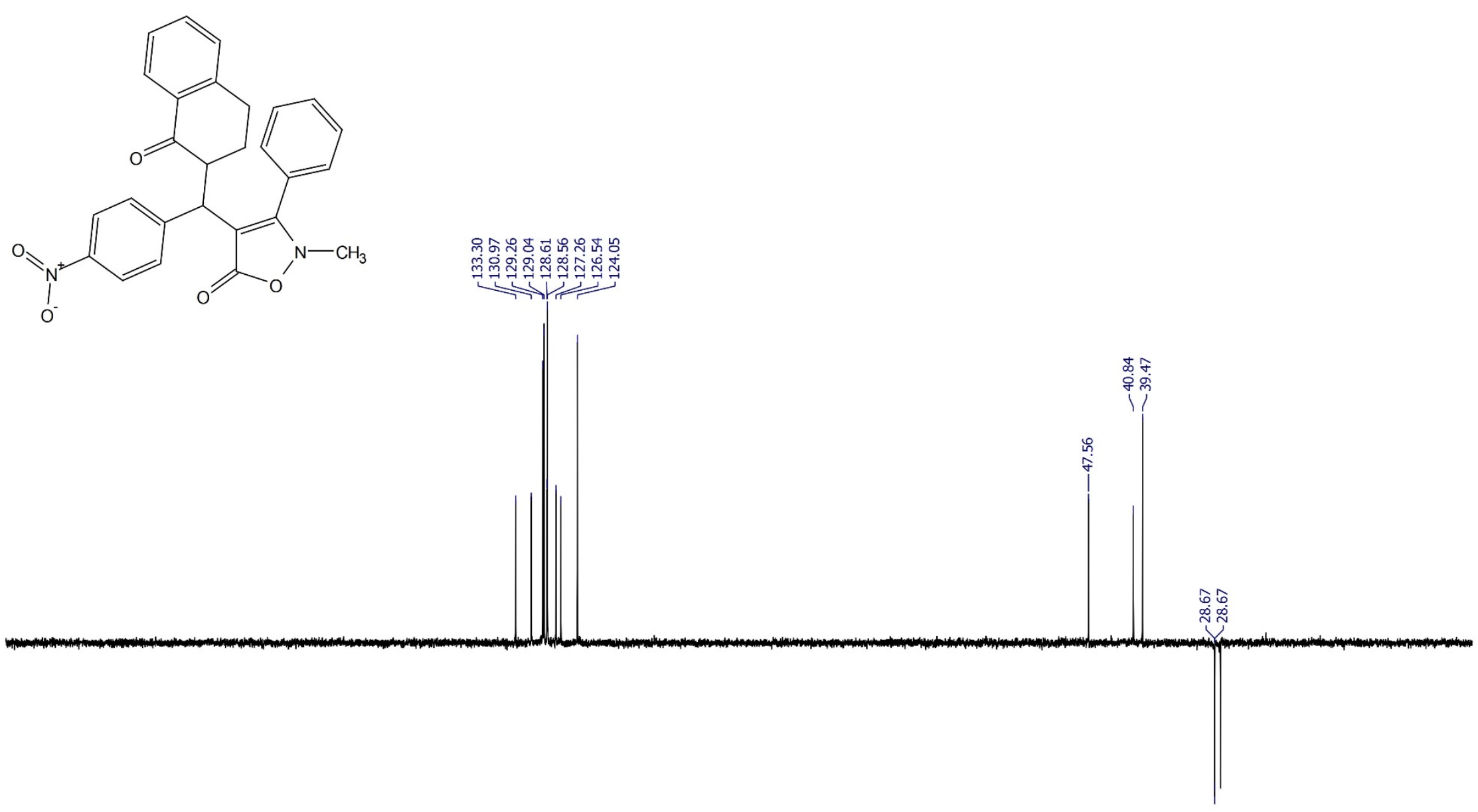


Methyl 4-(4-methoxyphenyl)-2-phenyl-5,6,7,8-tetrahydroquinoline-3-carboxylate 7a, ${ }^{1} \mathrm{H} \mathrm{NMR}, 400 \mathrm{MHz}, \mathrm{CDCl}_{3}$
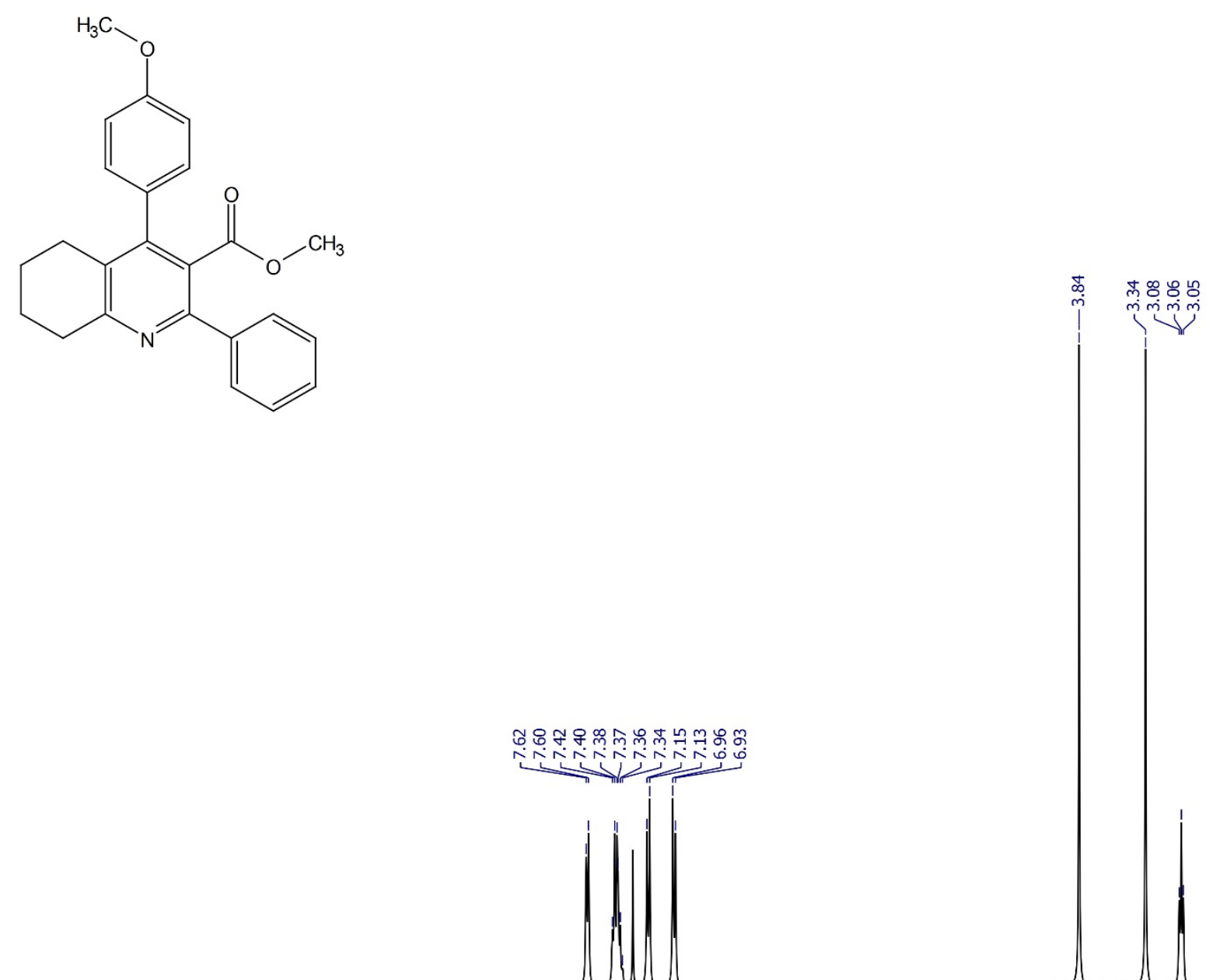

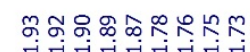

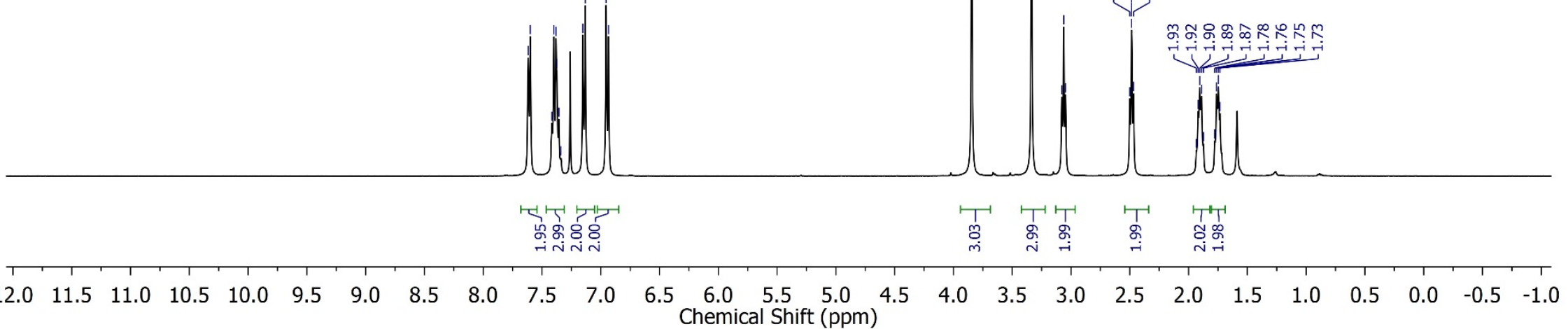


Methyl 4-(4-methoxyphenyl)-2-phenyl-5,6,7,8-tetrahydroquinoline-3-carboxylate $7 \mathrm{a},{ }^{13} \mathrm{C}\left\{{ }^{1} \mathrm{H}\right\} \mathrm{NMR}, 100 \mathrm{MHz}^{\mathrm{CDCl}} \mathrm{C}_{3}$
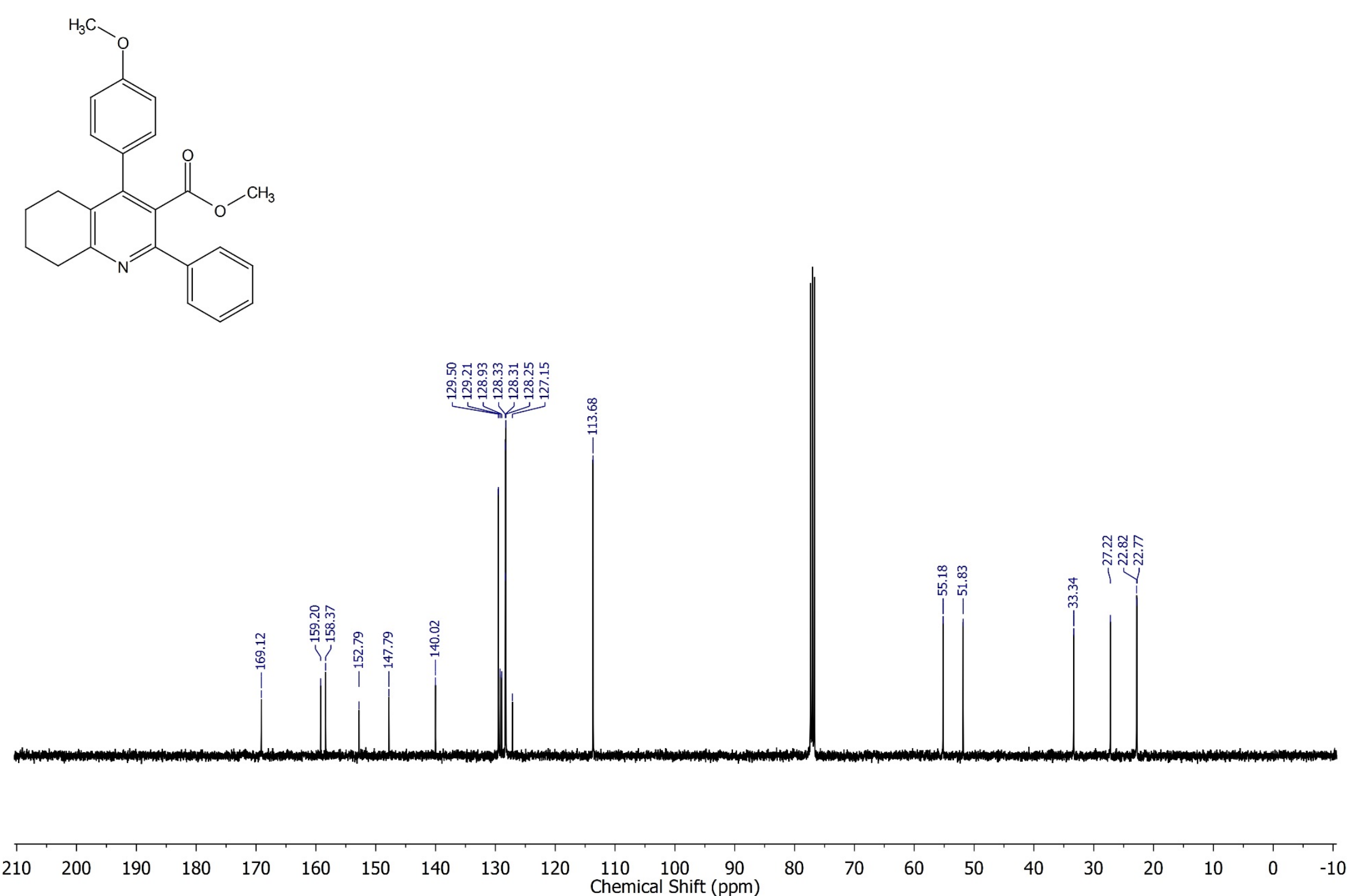
Methyl 4-(4-methoxyphenyl)-2-phenyl-5,6,7,8-tetrahydroquinoline-3-carboxylate 7a, DEPT, $100 \mathrm{MHz}, \mathrm{CDCl}_{3}$

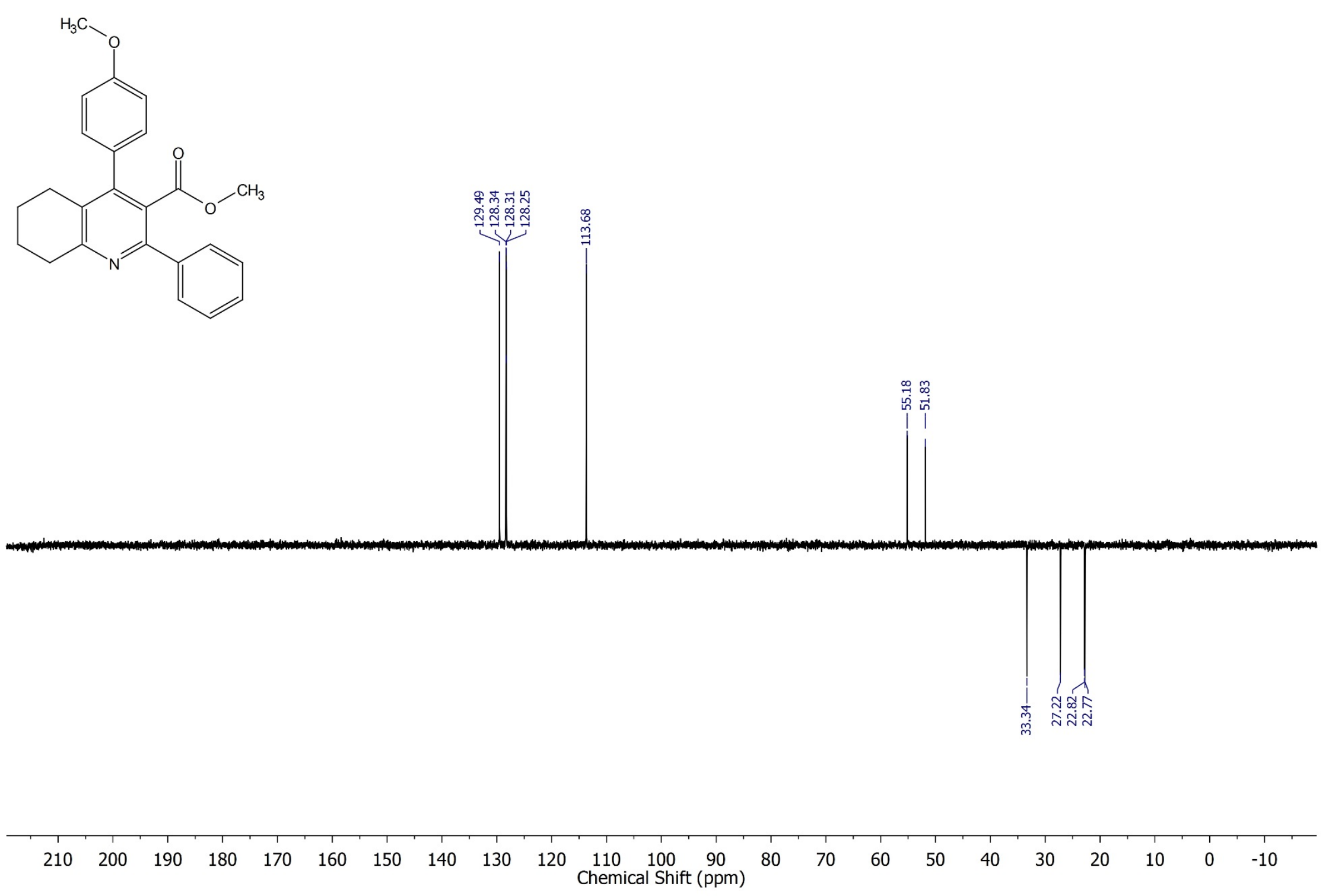


Methyl 4-(4-bromophenyl)-2-phenyl-5,6,7,8-tetrahydroquinoline-3-carboxylate $7 \mathrm{~b},{ }^{1} \mathrm{H}$ NMR, $400 \mathrm{MHz}, \mathrm{CDCl}_{3}$
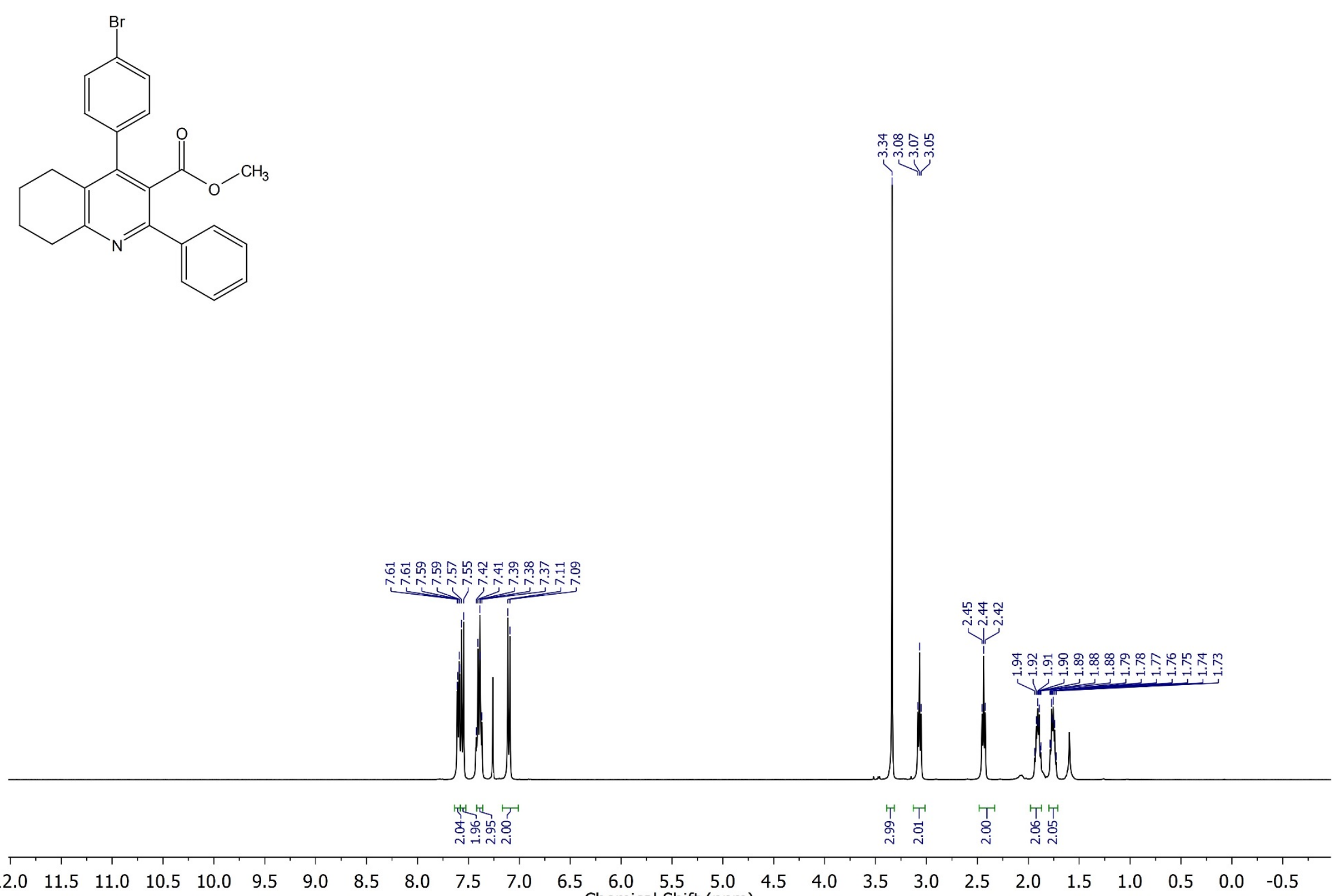
Methyl 4-(4-bromophenyl)-2-phenyl-5,6,7,8-tetrahydroquinoline-3-carboxylate $7 \mathrm{~b},{ }^{13} \mathrm{C}\left\{{ }^{1} \mathrm{H}\right\}$ NMR, $100 \mathrm{MHz}, \mathrm{CDCl}_{3}$
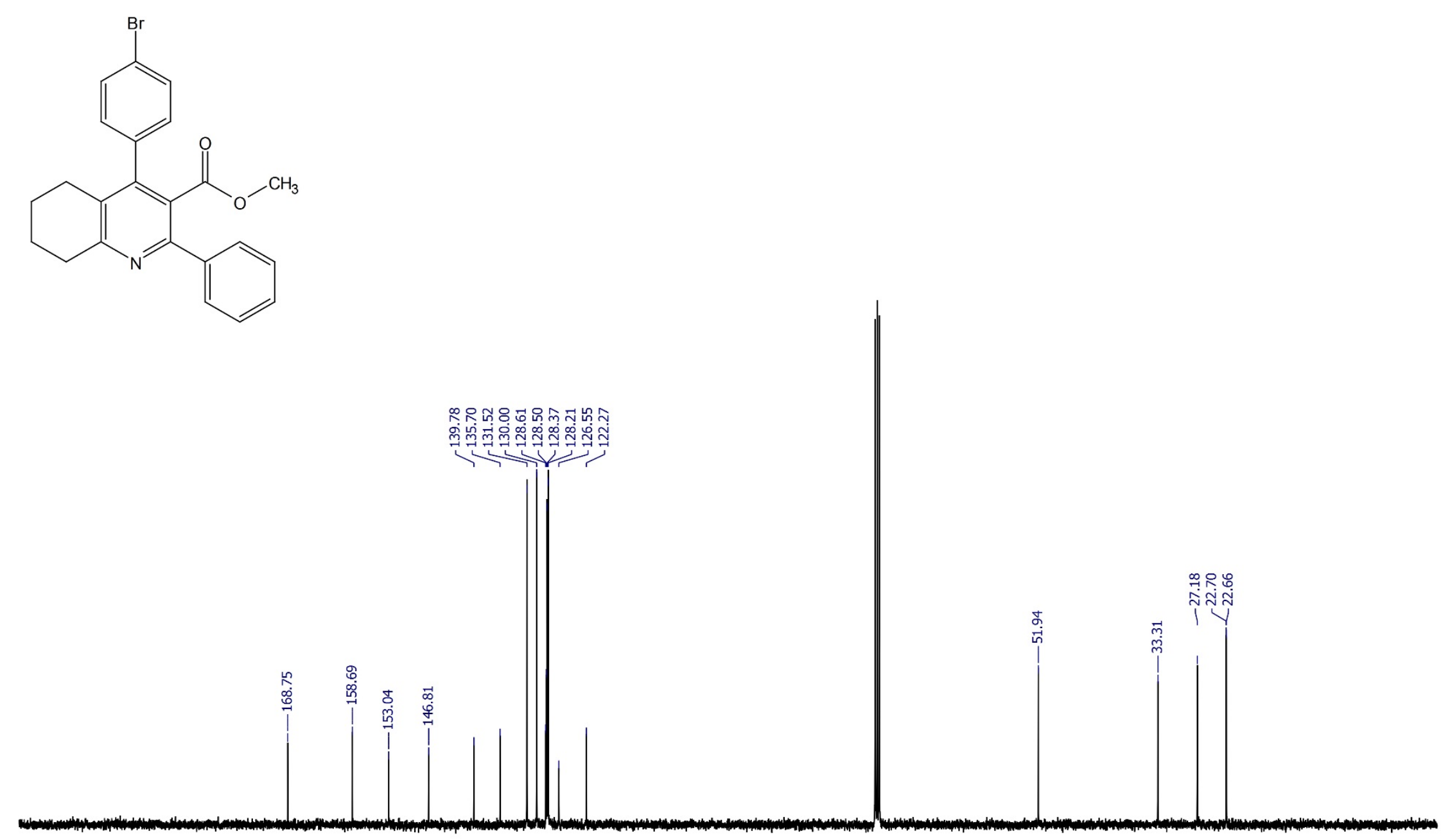

210 
Methyl 4-(4-bromophenyl)-2-phenyl-5,6,7,8-tetrahydroquinoline-3-carboxylate 7b, DEPT, $100 \mathrm{MHz}, \mathrm{CDCl}_{3}$
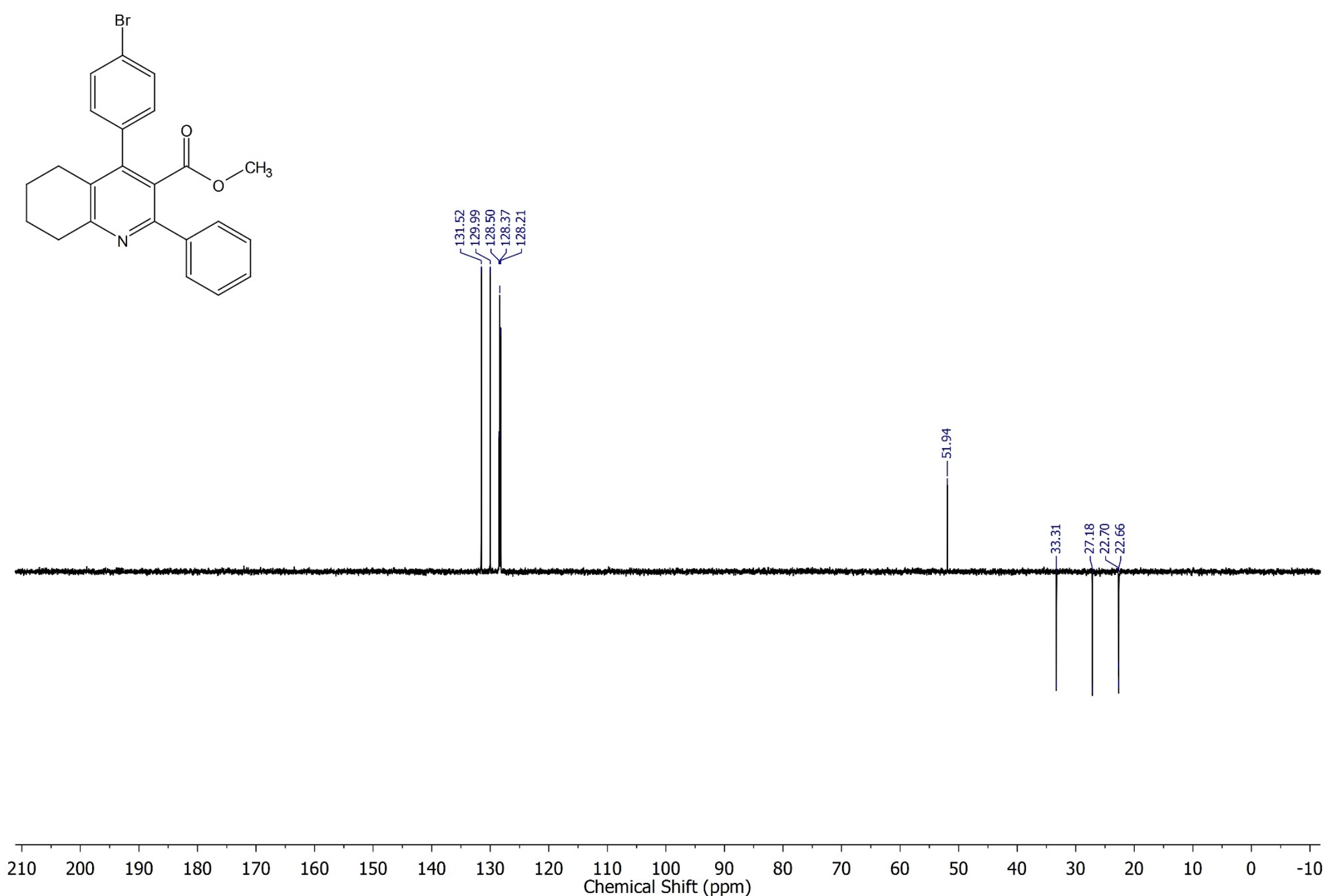
Methyl 4-(4-nitrophenyl)-2-phenyl-5,6,7,8-tetrahydroquinoline-3-carboxylate 7c, ${ }^{1} \mathrm{H} \mathrm{NMR}, 400 \mathrm{MHz}, \mathrm{CDCl}_{3}$
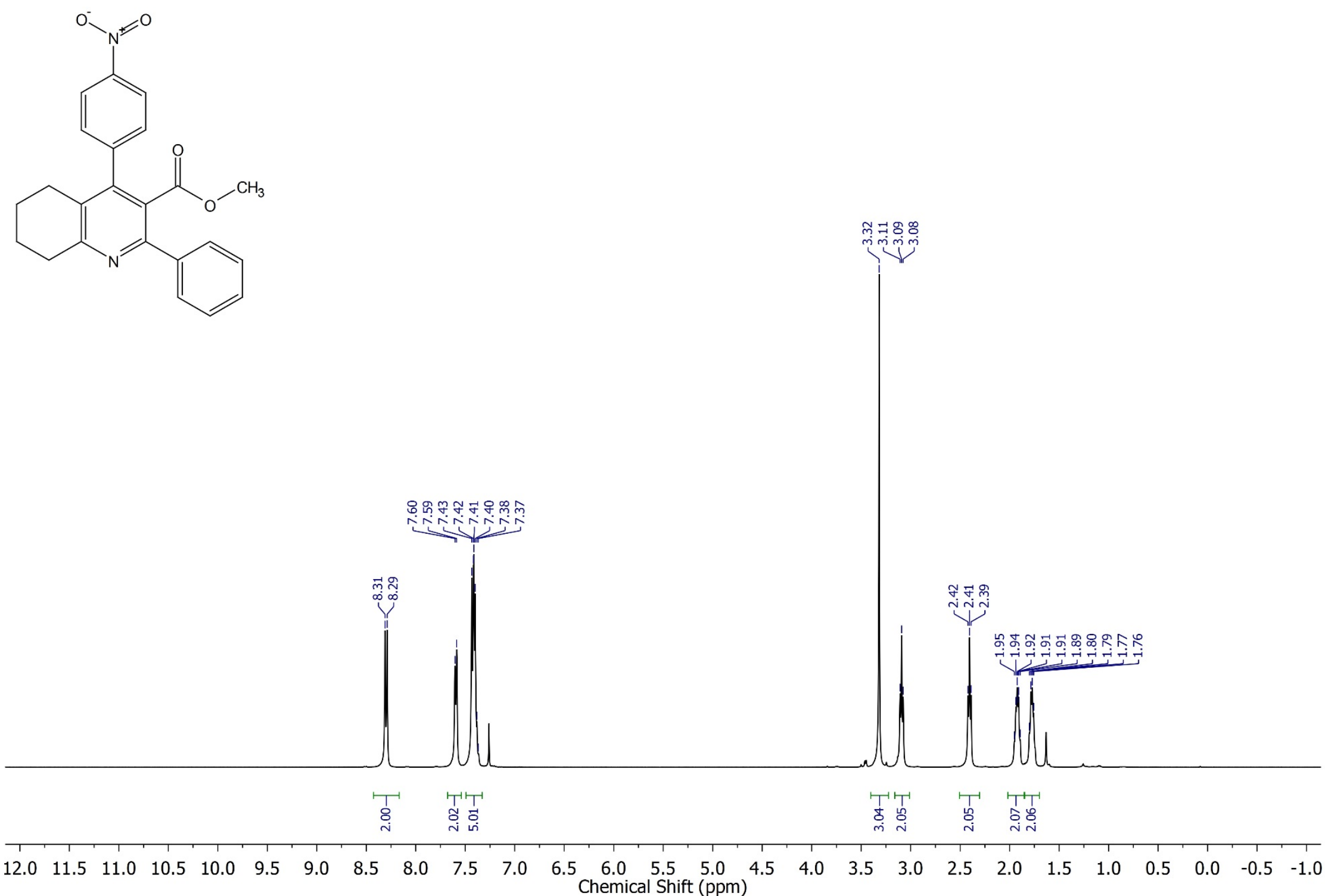
Methyl 4-(4-nitrophenyl)-2-phenyl-5,6,7,8-tetrahydroquinoline-3-carboxylate $7 \mathrm{c},{ }^{13} \mathrm{C}\left\{{ }^{1} \mathrm{H}\right\} \mathrm{NMR}, 100 \mathrm{MHz} \mathrm{CDCl}_{3}$
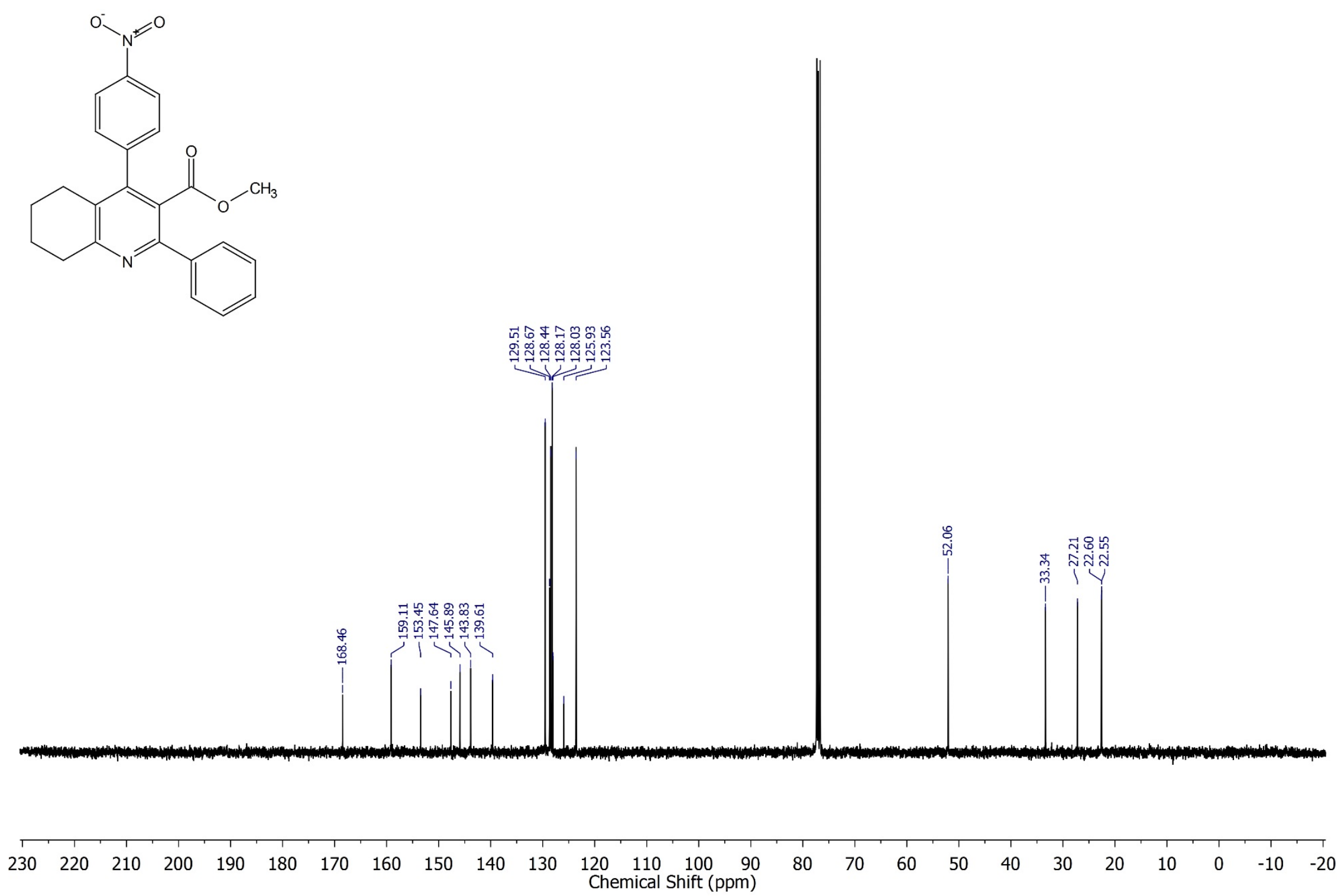
Methyl 4-(4-nitrophenyl)-2-phenyl-5,6,7,8-tetrahydroquinoline-3-carboxylate 7c, DEPT, $100 \mathrm{MHz}, \mathrm{CDCl}_{3}$

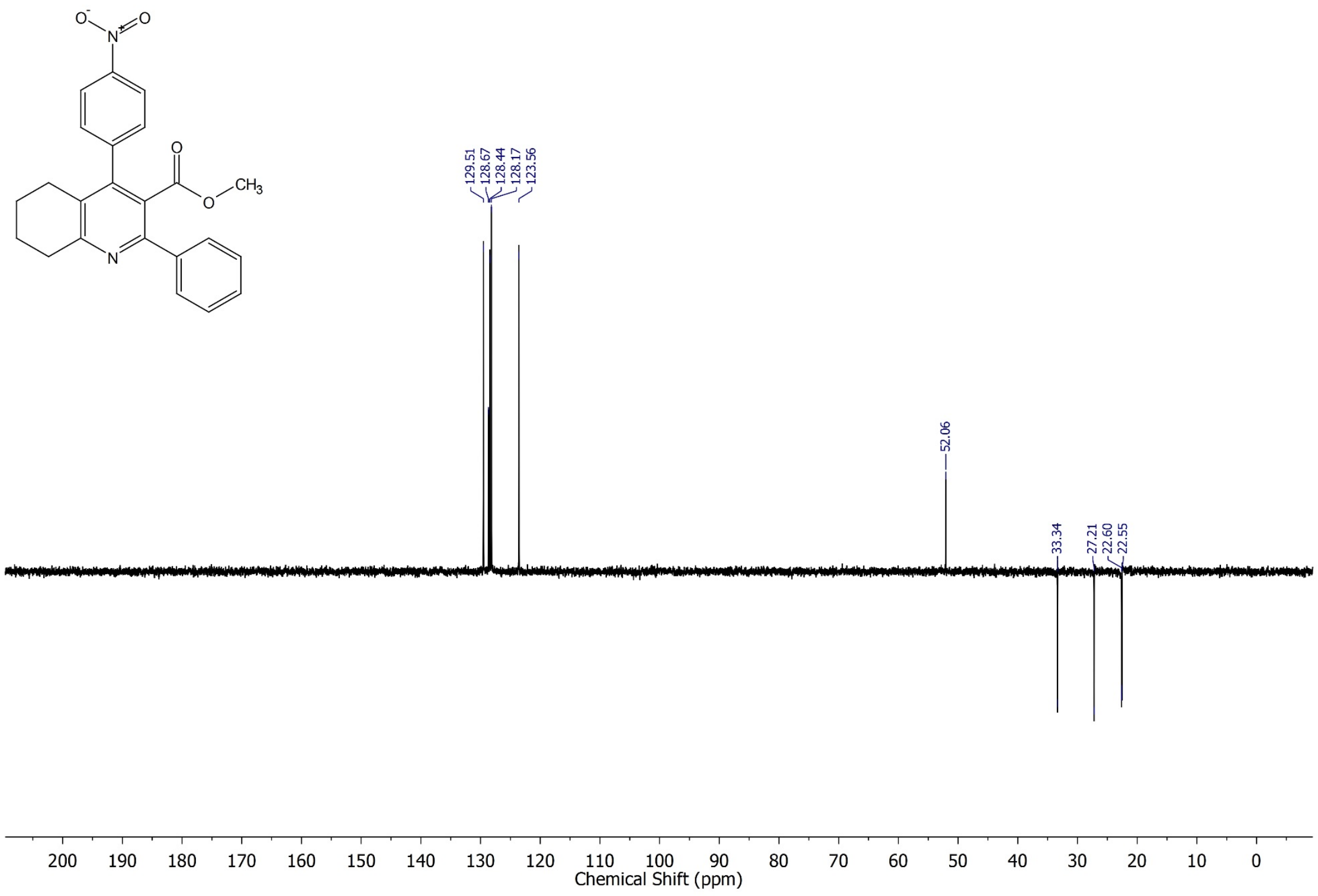


Methyl 2-(4-nitrophenyl)-4-(p-tolyl)-5,6,7,8-tetrahydroquinoline-3-carboxylate 7d, ${ }^{1} \mathrm{H} \mathrm{NMR}, 400 \mathrm{MHz}, \mathrm{CDCl}_{3}$
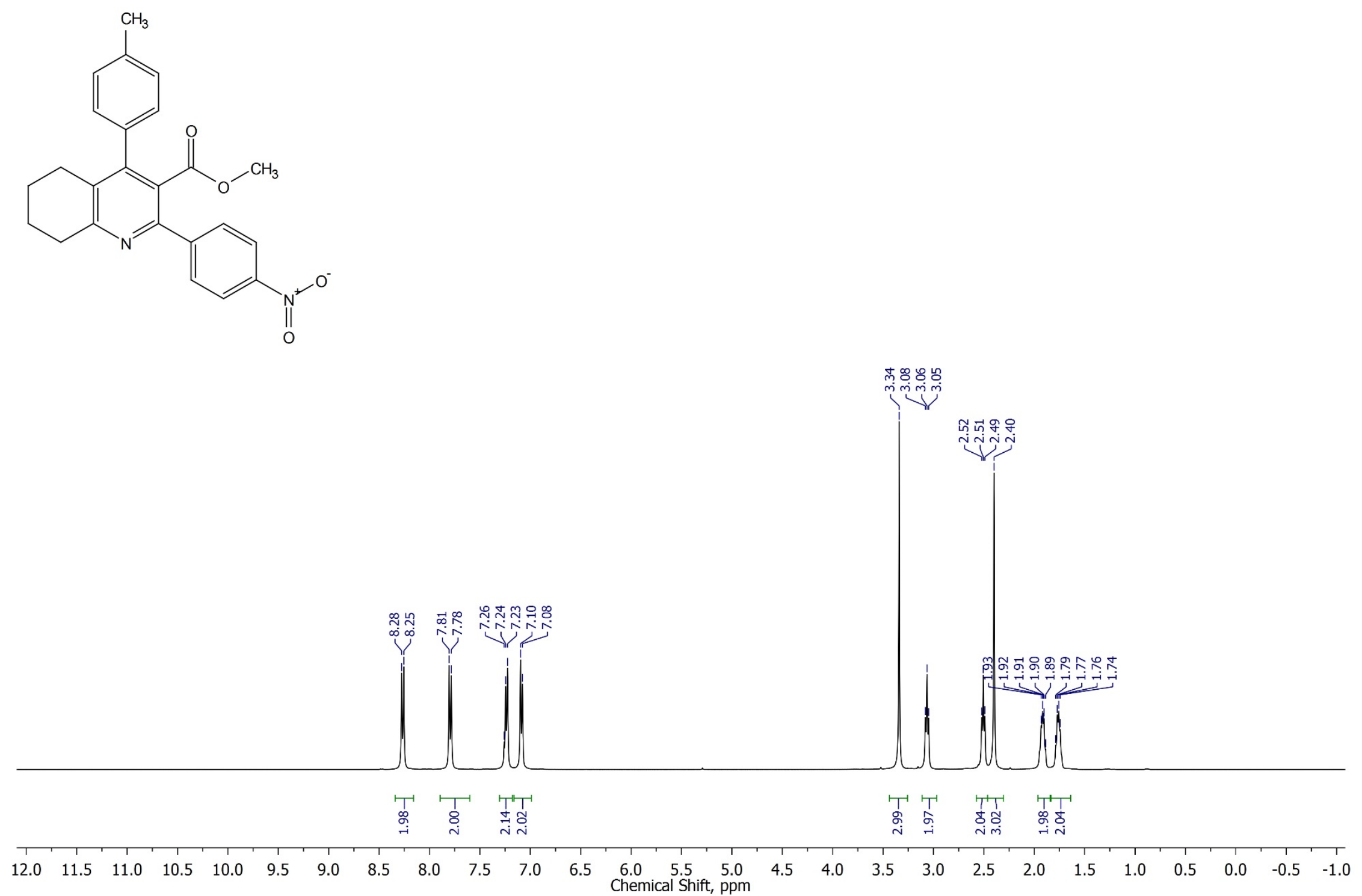
Methyl 2-(4-nitrophenyl)-4-(p-tolyl)-5,6,7,8-tetrahydroquinoline-3-carboxylate $7 \mathrm{~d},{ }^{13} \mathrm{C}\left\{{ }^{1} \mathrm{H}\right\} \mathrm{NMR}, 100 \mathrm{MHz}, \mathrm{CDCl}_{3}$
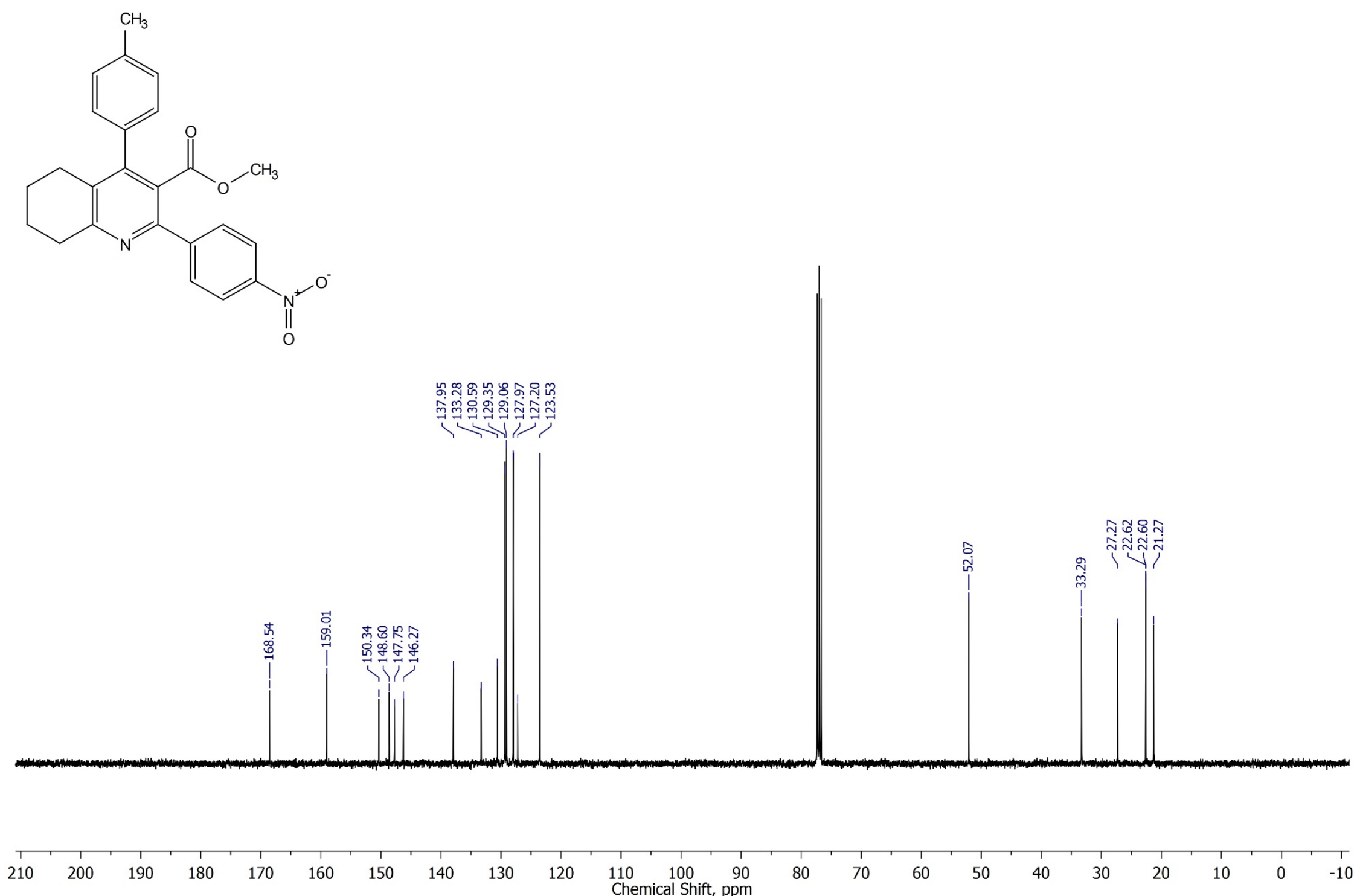
Methyl 2-(4-nitrophenyl)-4-(p-tolyl)-5,6,7,8-tetrahydroquinoline-3-carboxylate 7d, DEPT, $100 \mathrm{MHz}, \mathrm{CDCl}_{3}$
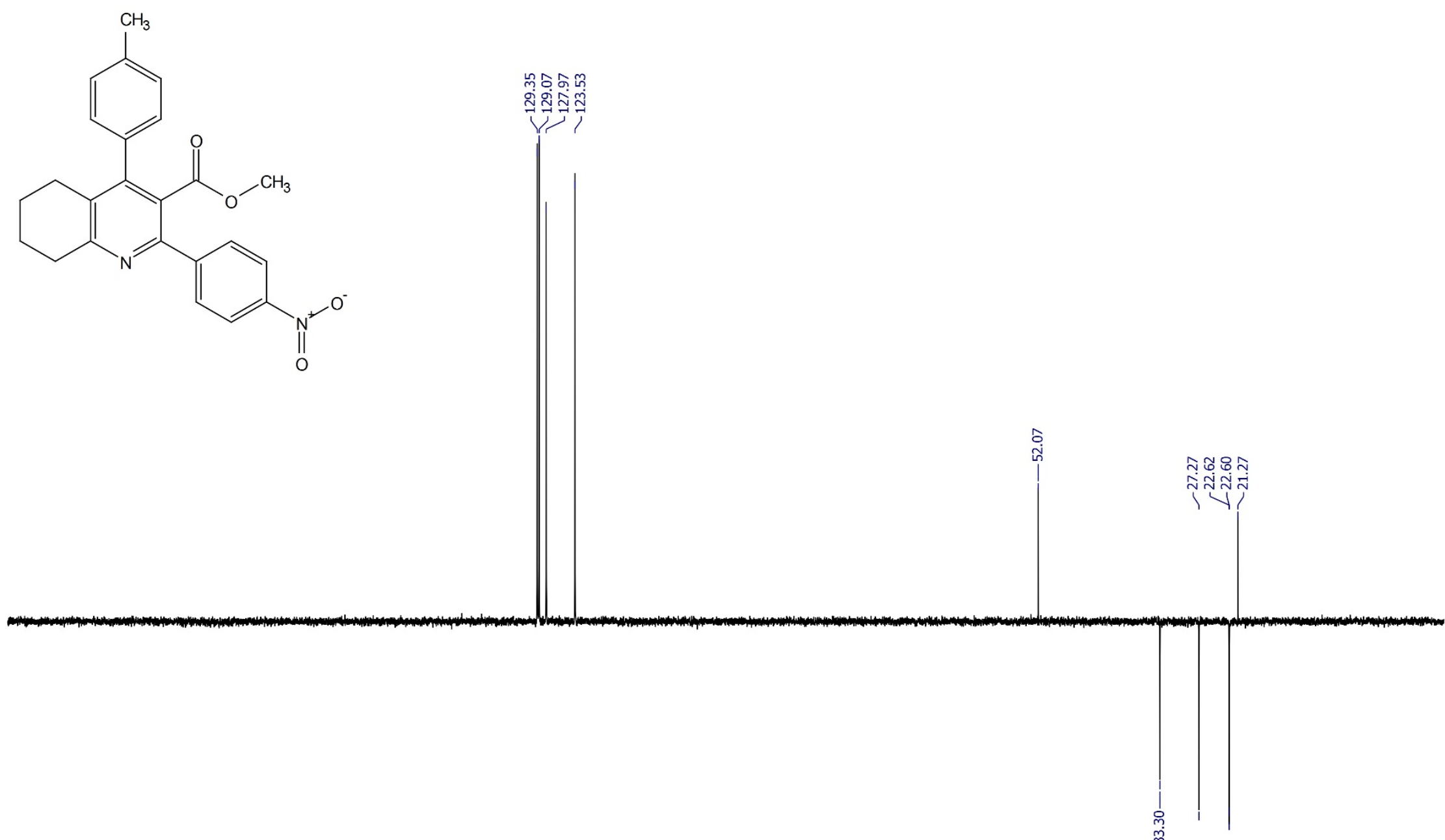

$210 \quad 200 \quad 190$ 
Methyl 4-(4-chlorophenyl)-2-(pyridin-2-yl)-5,6,7,8-tetrahydroquinoline-3-carboxylate 7e, ${ }^{1} \mathrm{H}$ NMR, $400 \mathrm{MHz}, \mathrm{CDCl}_{3}$
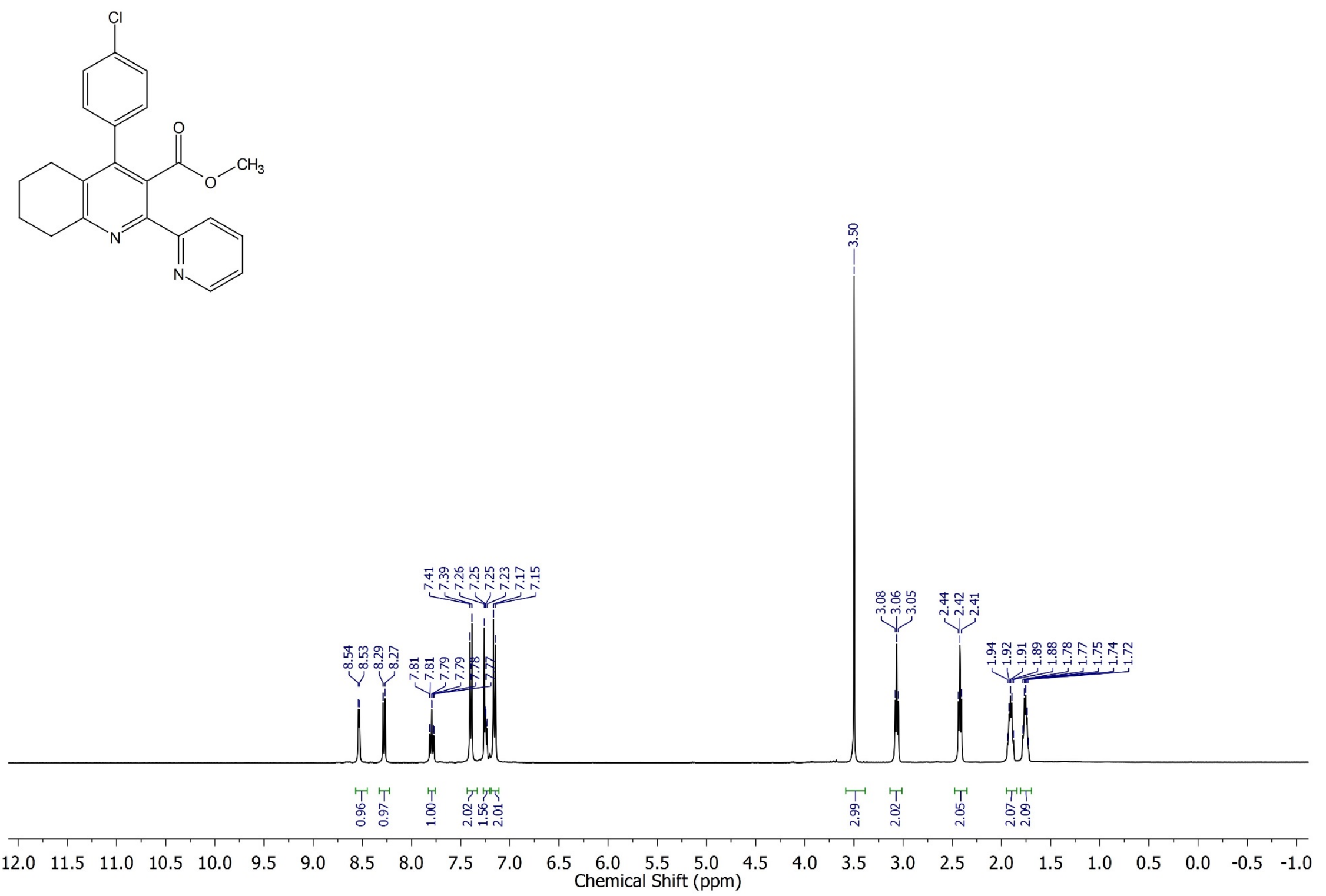
Methyl 4-(4-chlorophenyl)-2-(pyridin-2-yl)-5,6,7,8-tetrahydroquinoline-3-carboxylate $7 \mathrm{e},{ }^{13} \mathrm{C}\left\{{ }^{1} \mathrm{H}\right\} \mathrm{NMR}, 100 \mathrm{MHz}^{\mathrm{CDCl}} \mathrm{CD}_{3}$

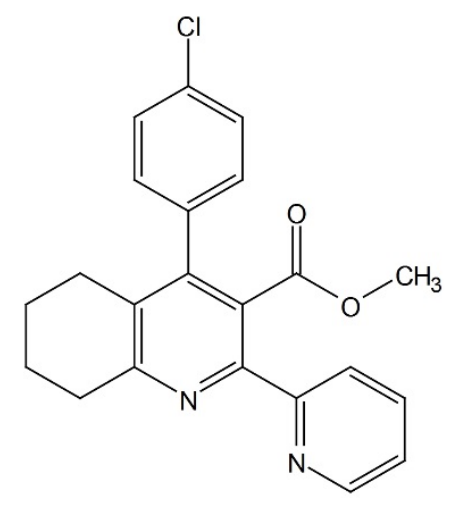

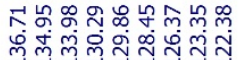

角
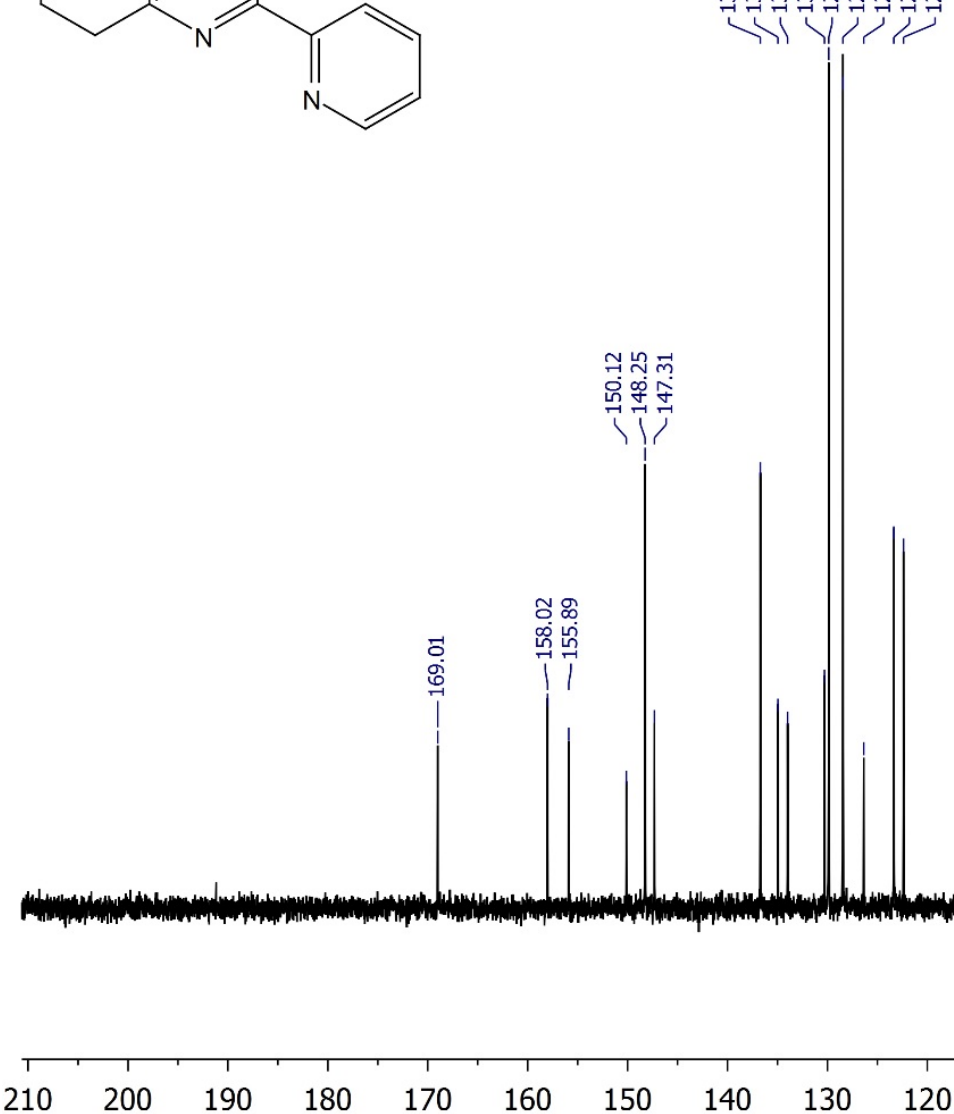

130

$20 \quad 110 \quad 100 \quad 90$

$80 \quad 70$

60

50

40

30

20

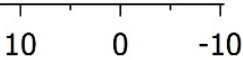


Methyl 4-(4-chlorophenyl)-2-(pyridin-2-yl)-5,6,7,8-tetrahydroquinoline-3-carboxylate 7e, DEPT, $100 \mathrm{MHz} \mathrm{CDCl}_{3}$

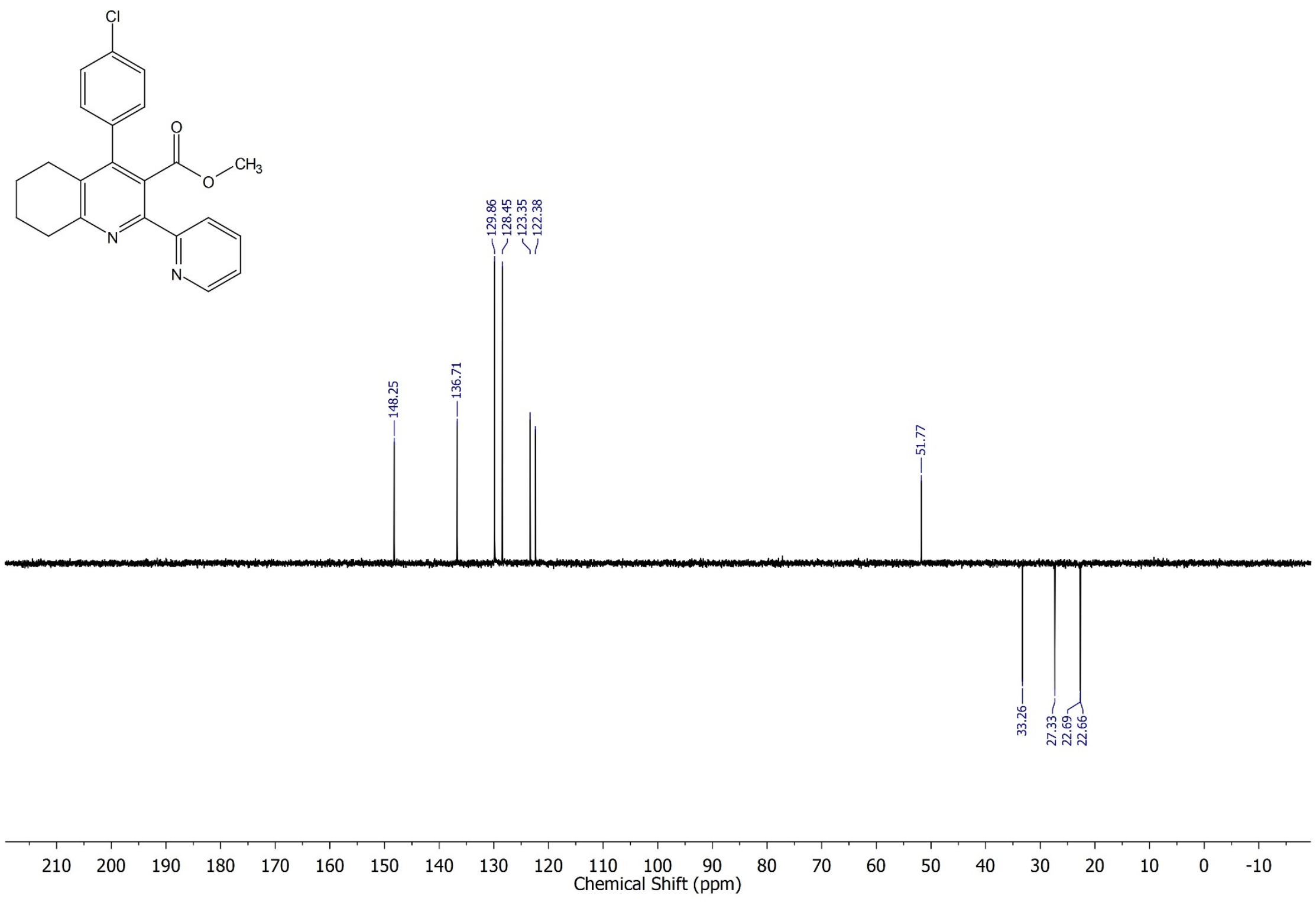


Methyl 4-(p-tolyl)-5,6,7,8-tetrahydro-[2,2'-biquinoline]-3-carboxylate 7f, ${ }^{1} \mathrm{H} \mathrm{NMR}, 400 \mathrm{MHz}, \mathrm{CDCl}_{3}$

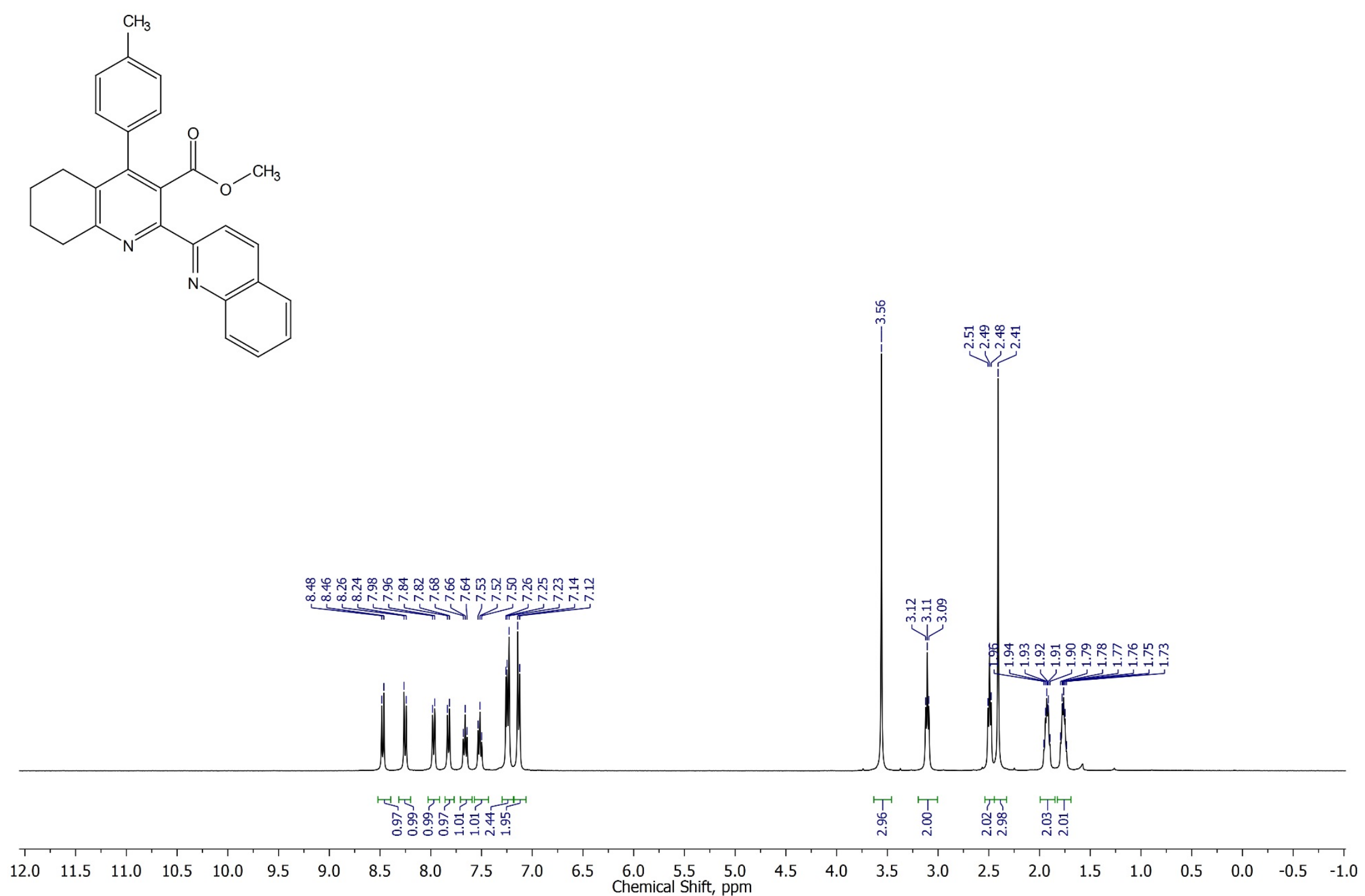


Methyl 4-(p-tolyl)-5,6,7,8-tetrahydro-[2,2'-biquinoline]-3-carboxylate 7f, ${ }^{13} \mathrm{C}\left\{{ }^{1} \mathrm{H}\right\} \mathrm{NMR}, 100 \mathrm{MHz}, \mathrm{CDCl}_{3}$

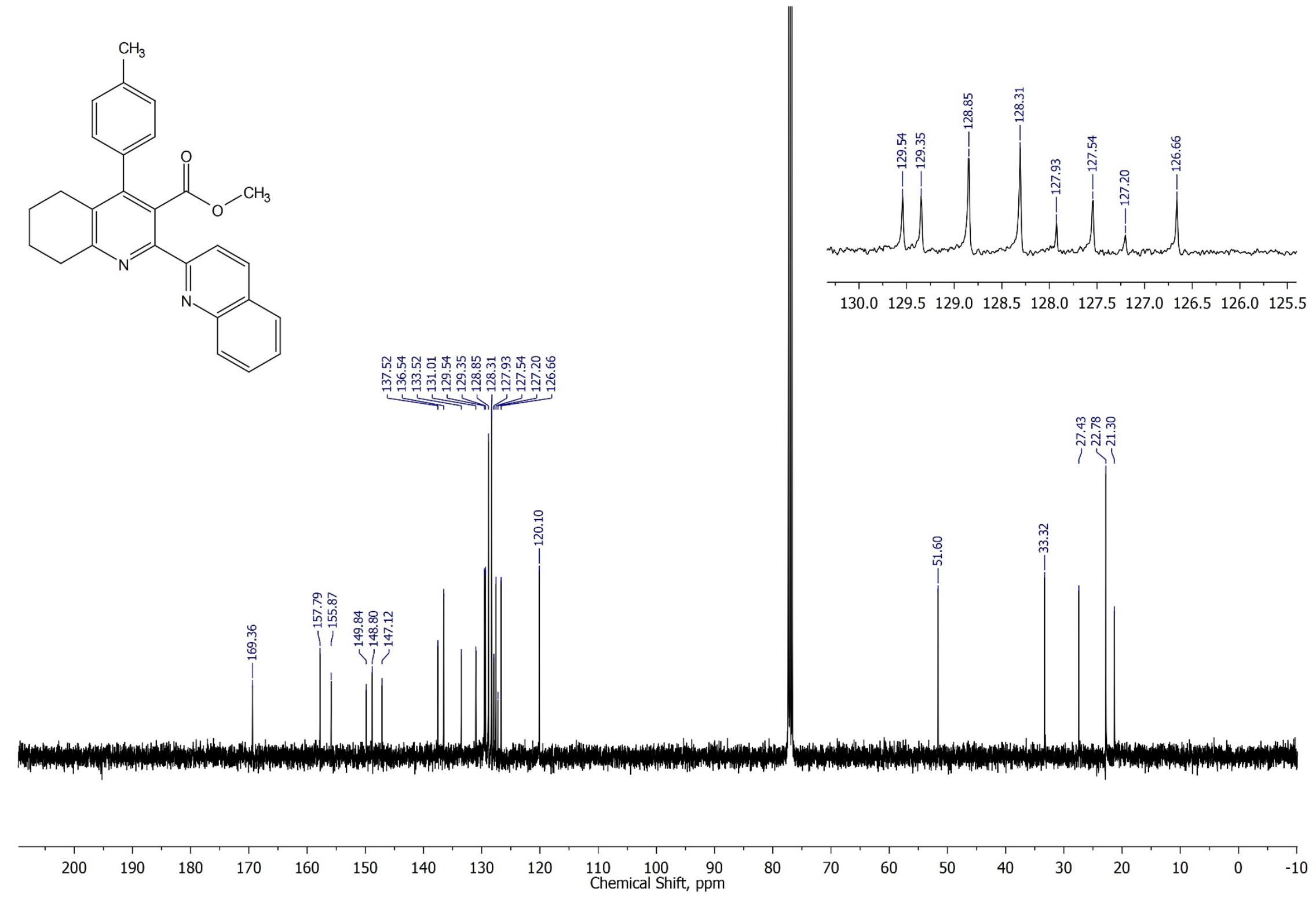


Methyl 4-(p-tolyl)-5,6,7,8-tetrahydro-[2,2'-biquinoline]-3-carboxylate 7f, DEPT, $100 \mathrm{MHz}, \mathrm{CDCl}_{3}$
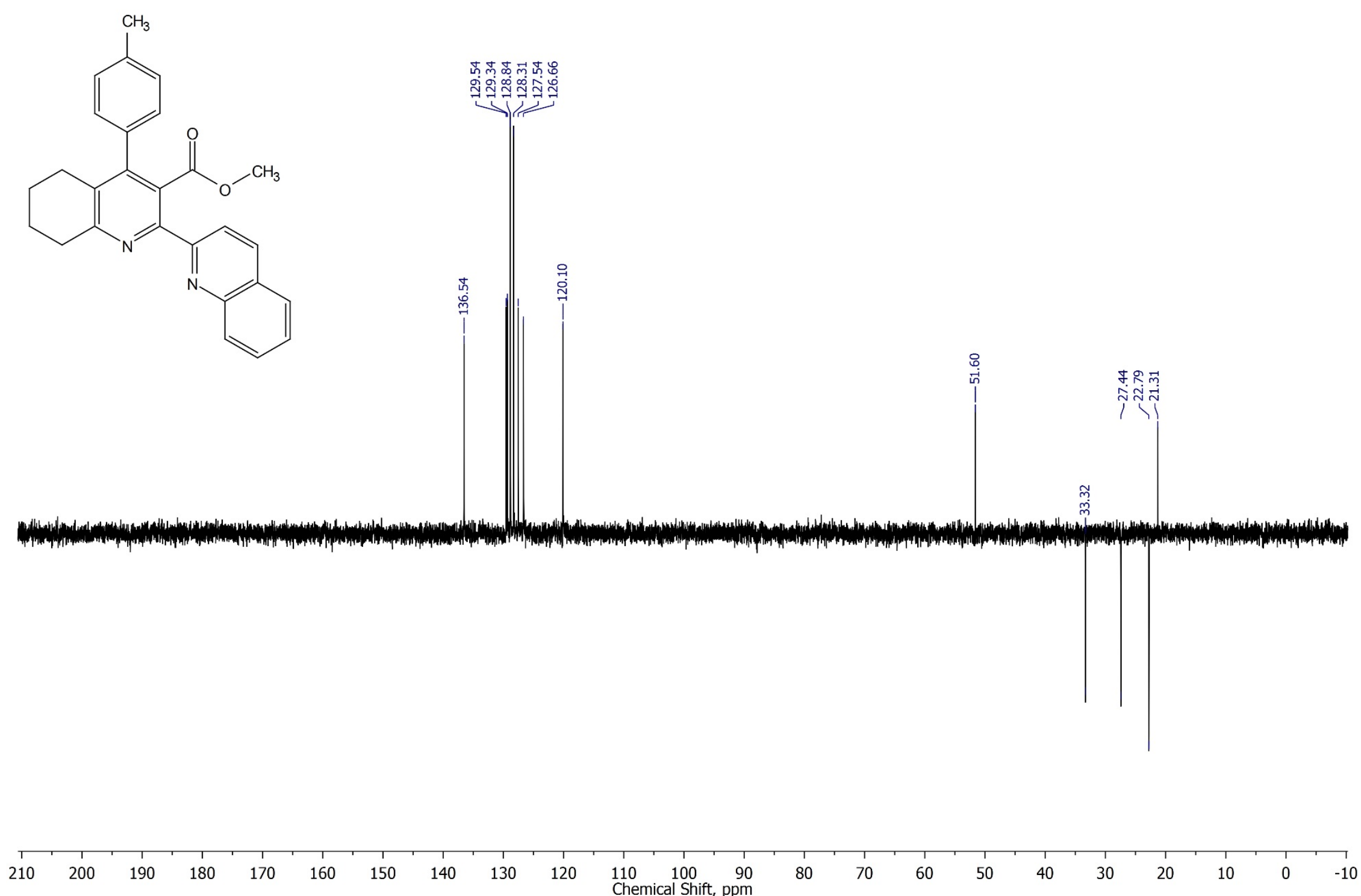
Methyl 4-(4-methoxyphenyl)-2-methyl-5,6,7,8-tetrahydroquinoline-3-carboxylate $7 \mathrm{~g},{ }^{1} \mathrm{H} \mathrm{NMR}, 400 \mathrm{MHz}, \mathrm{CDCl}_{3}$
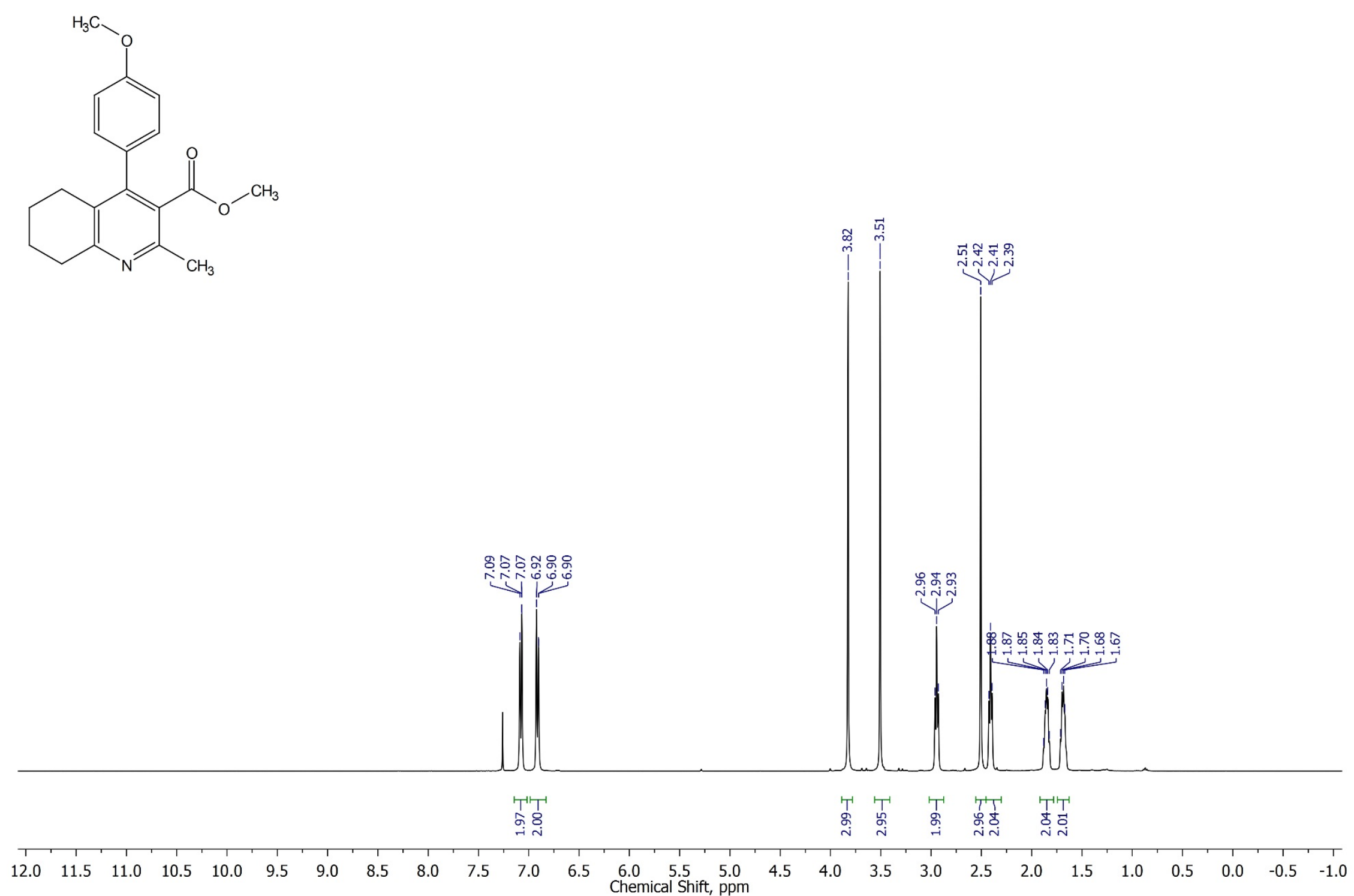
Methyl 4-(4-methoxyphenyl)-2-methyl-5,6,7,8-tetrahydroquinoline-3-carboxylate $7 \mathrm{~g},{ }^{13} \mathrm{C}\left\{{ }^{1} \mathrm{H}\right\} \mathrm{NMR}, 100 \mathrm{MHz}^{\mathrm{CDCl}} \mathrm{C}_{3}$
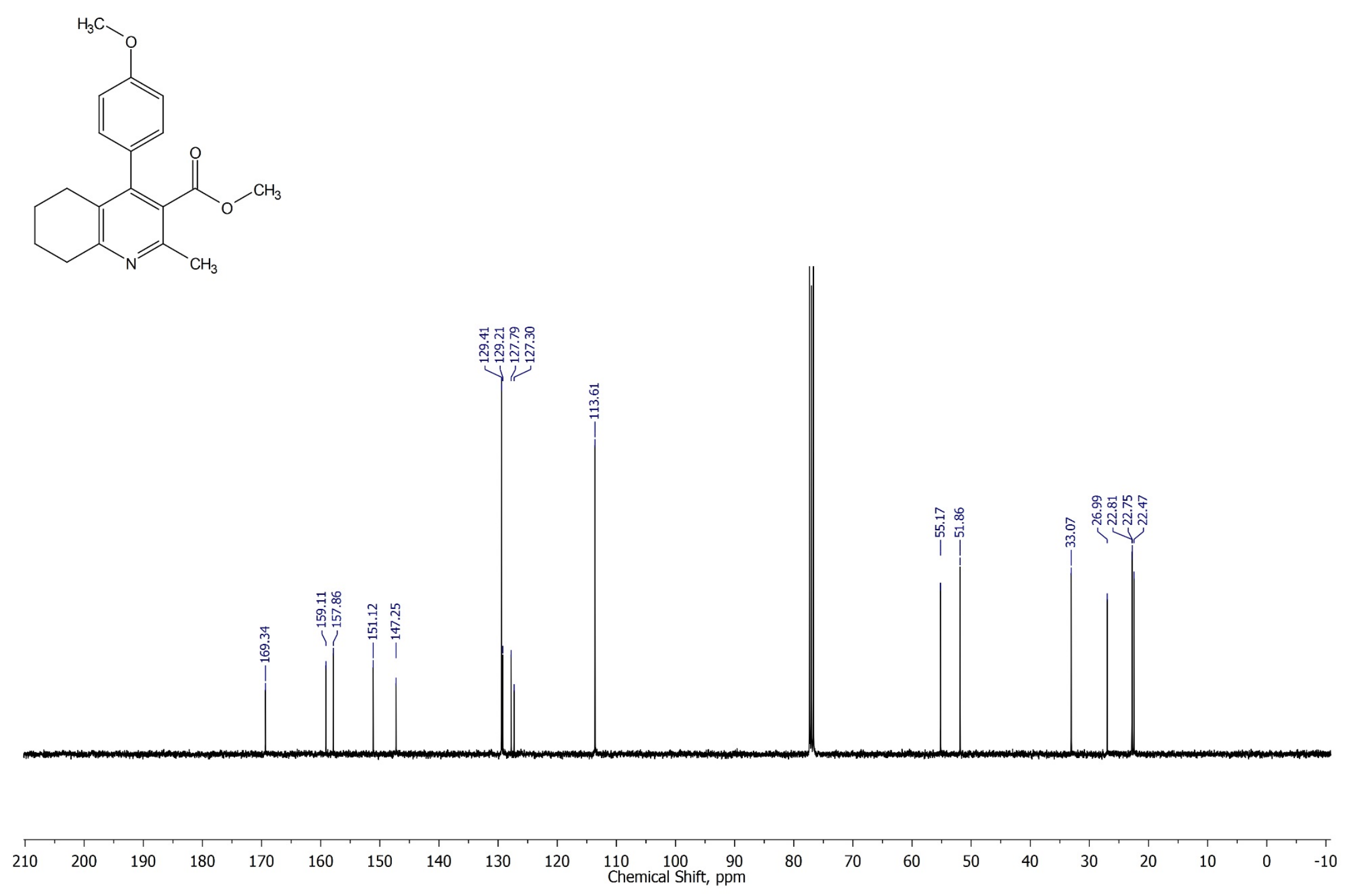
Methyl 4-(4-methoxyphenyl)-2-methyl-5,6,7,8-tetrahydroquinoline-3-carboxylate 7g, DEPT, $100 \mathrm{MHz}, \mathrm{CDCl}_{3}$
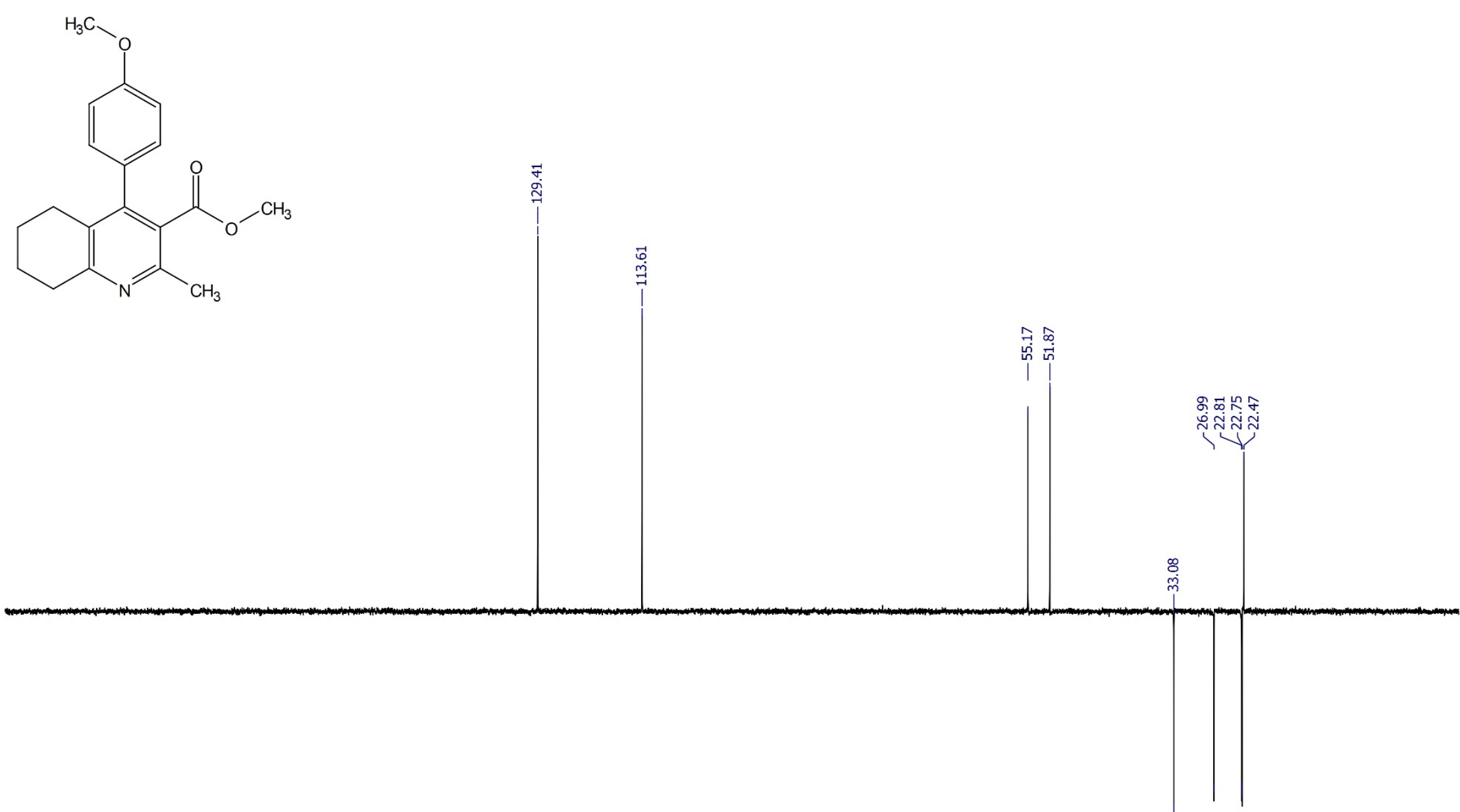

$210 \quad 200 \quad 190 \quad 180$

$170 \quad 160$ 
Methyl 4-(4-methoxyphenyl)-2-phenyl-6,7,8,9-tetrahydro-5H-cyclohepta[b]pyridine-3-carboxylate 7h, ${ }^{1} \mathrm{H} \mathrm{NMR}, 400 \mathrm{MHz}, \mathrm{CDCl}_{3}$

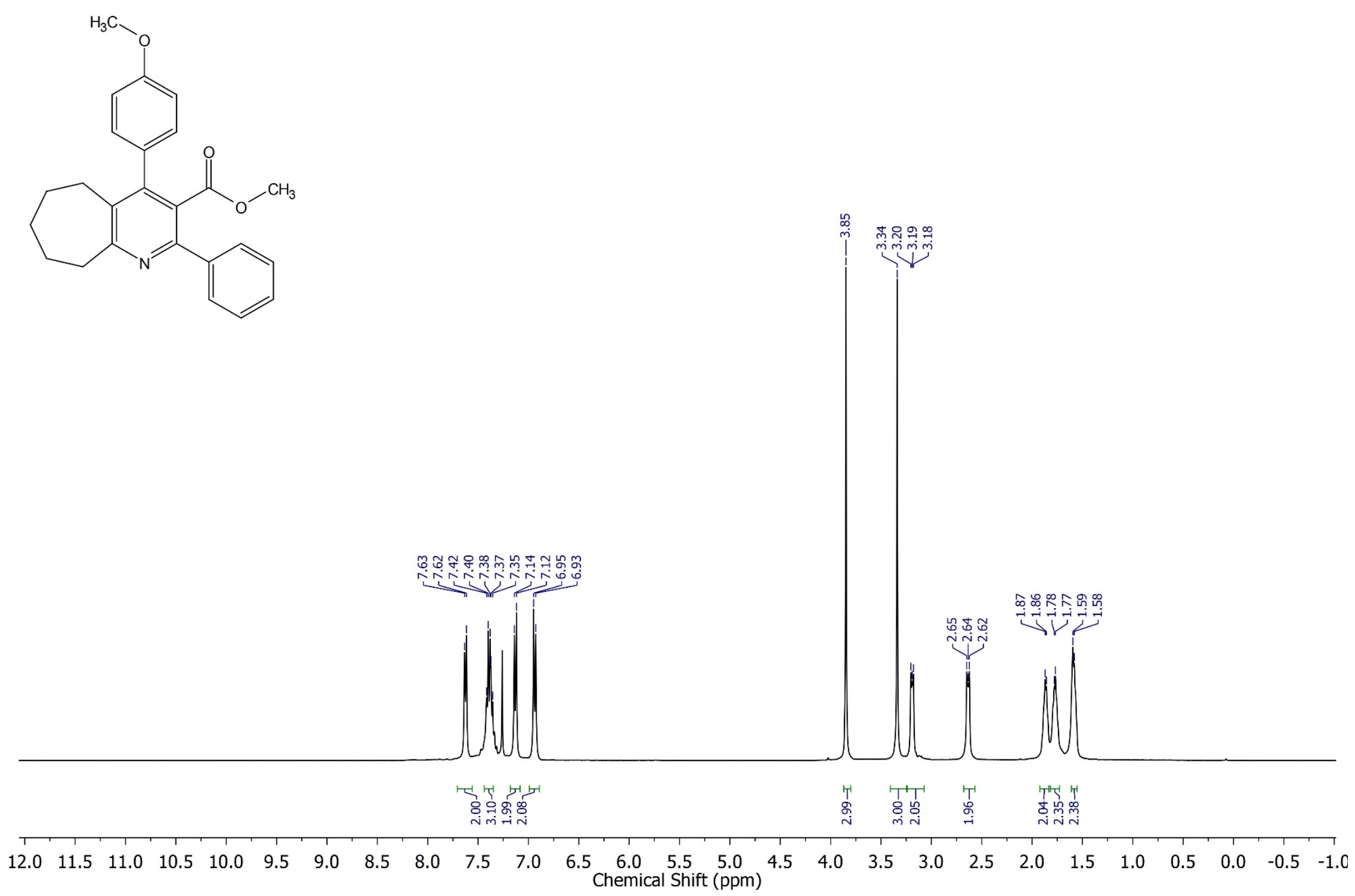


Methyl 4-(4-methoxyphenyl)-2-phenyl-6,7,8,9-tetrahydro-5H-cyclohepta[b]pyridine-3-carboxylate $7 \mathrm{~h},{ }^{13} \mathrm{C}\left\{{ }^{1} \mathrm{H}\right\}$ NMR, 100 $\mathrm{MHz}, \mathrm{CDCl}{ }_{3}$

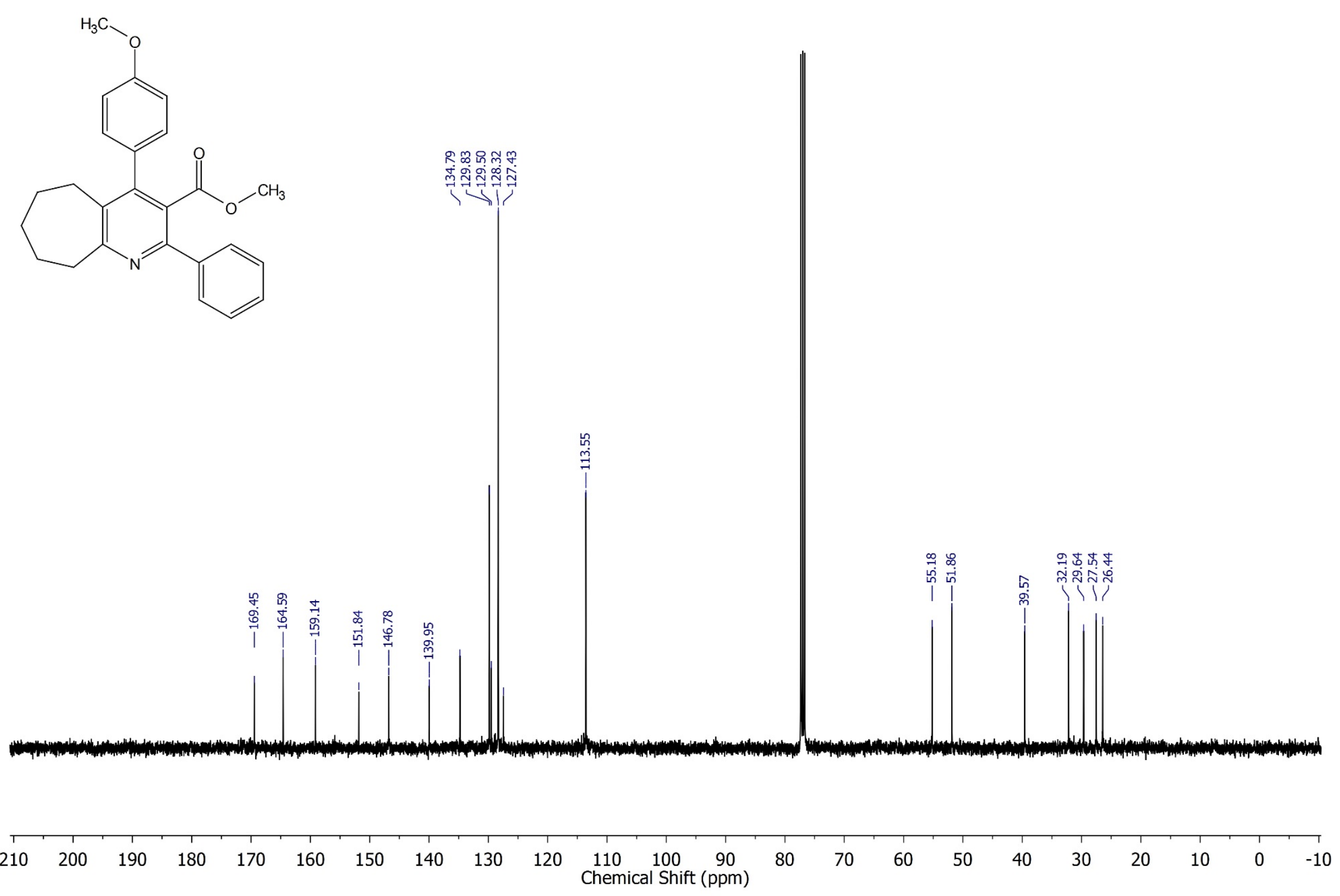


Methyl 4-(4-methoxyphenyl)-2-phenyl-6,7,8,9-tetrahydro-5H-cyclohepta[b]pyridine-3-carboxylate 7h, DEPT, $100 \mathrm{MHz}^{\mathrm{CDCl}} \mathrm{CH}_{3}$

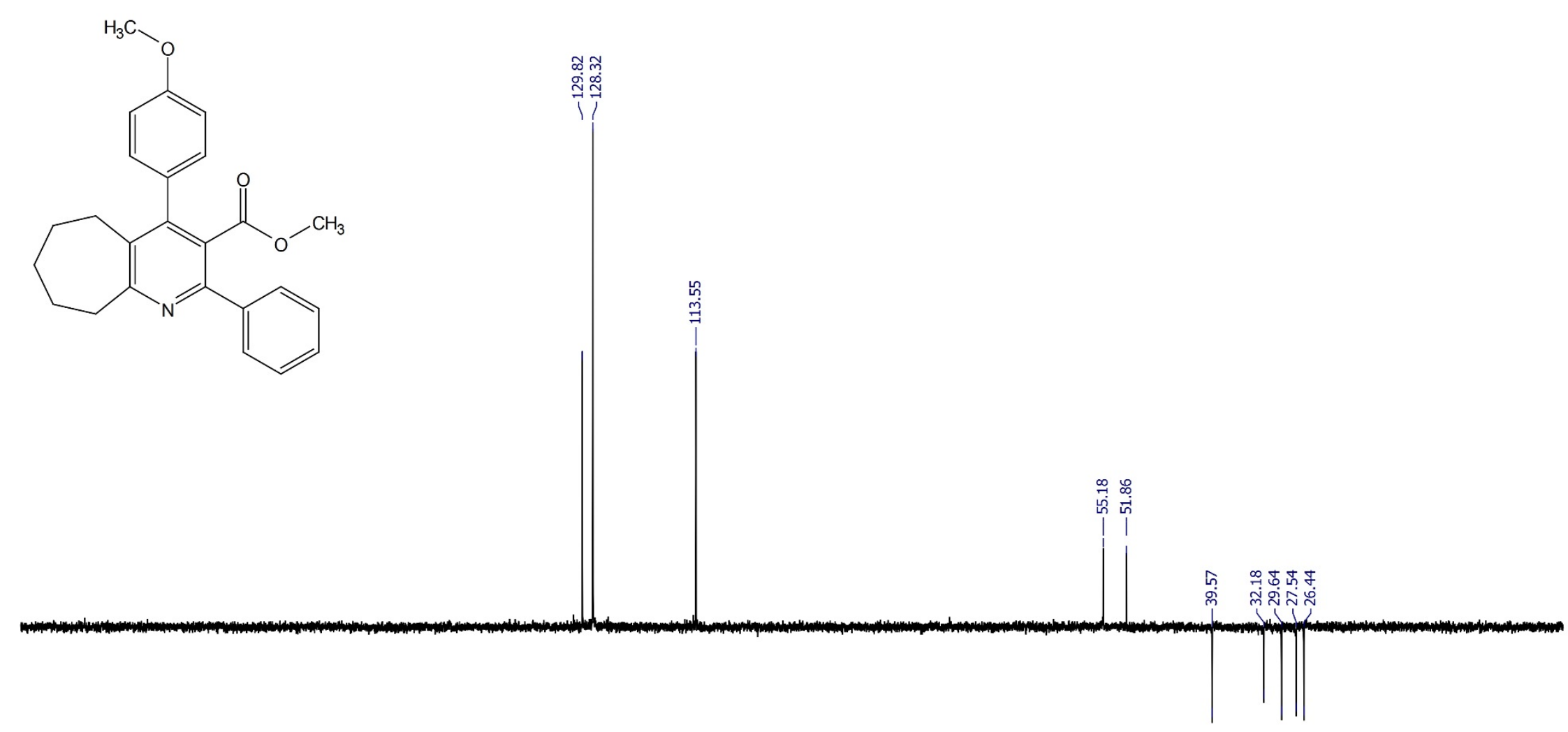

\begin{tabular}{rrrrrrrrrrrrrrrrrrrrrrrrrrrr}
\hline & 210 & 200 & 190 & 180 & 170 & 160 & 150 & 140 & 130 & 120 & $\begin{array}{c}110 \\
\text { Chemical Shift (ppm) }\end{array}$ & 80 & 70 & 60 & 50 & 40 & 30 & 20 & 10 & 0 & -10
\end{tabular}


Methyl 4-(4-nitrophenyl)-2-phenyl-5,6-dihydrobenzo[h]quinoline-3-carboxylate $7 \mathrm{i},{ }^{1} \mathrm{H} \mathrm{NMR}, 400 \mathrm{MHz}, \mathrm{CDCl}_{3}$

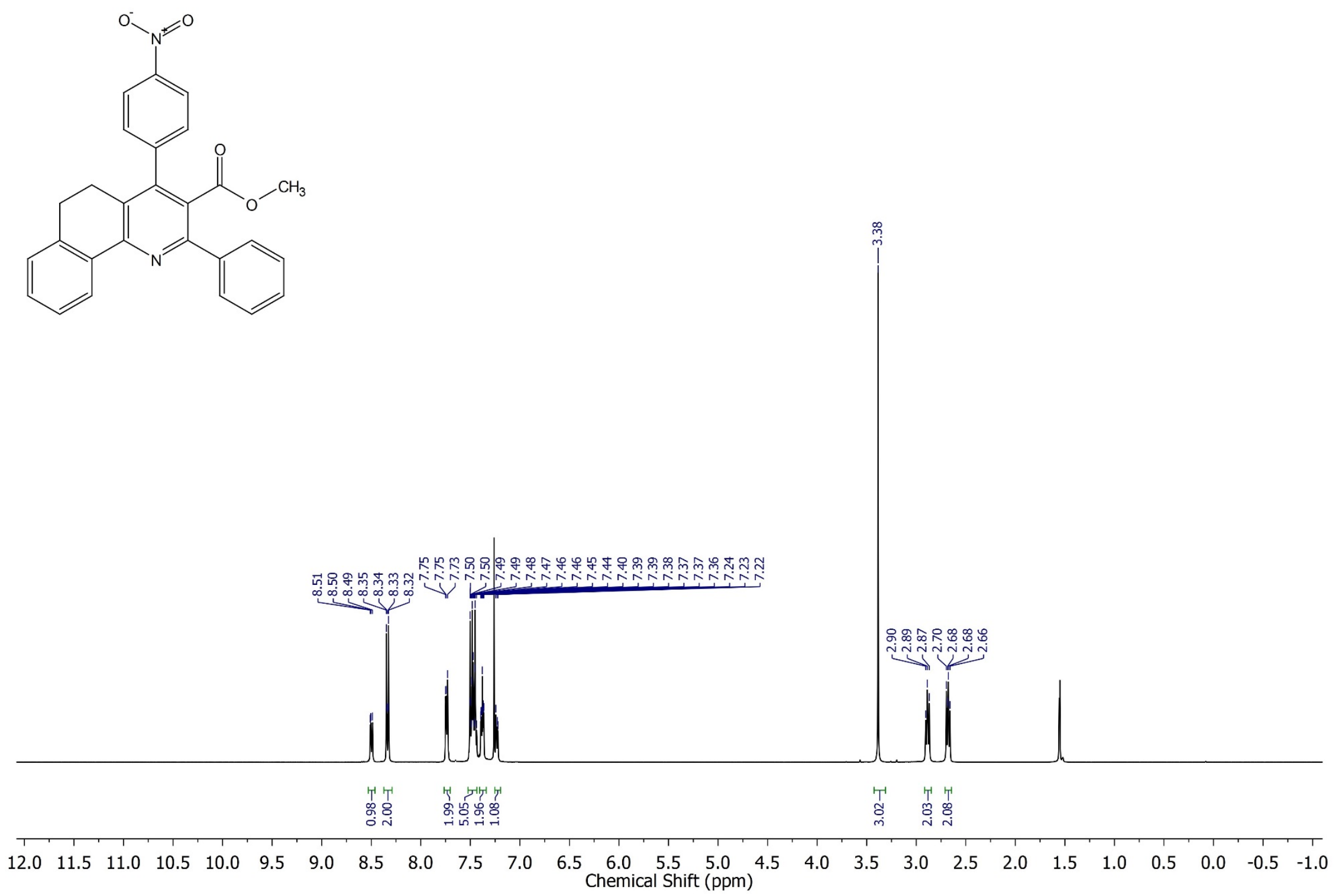


Methyl 4-(4-nitrophenyl)-2-phenyl-5,6-dihydrobenzo[h]quinoline-3-carboxylate $7 \mathrm{i},{ }^{13} \mathrm{C}\left\{{ }^{1} \mathrm{H}\right\}$ NMR, $100 \mathrm{MHz}, \mathrm{CDCl}_{3}$

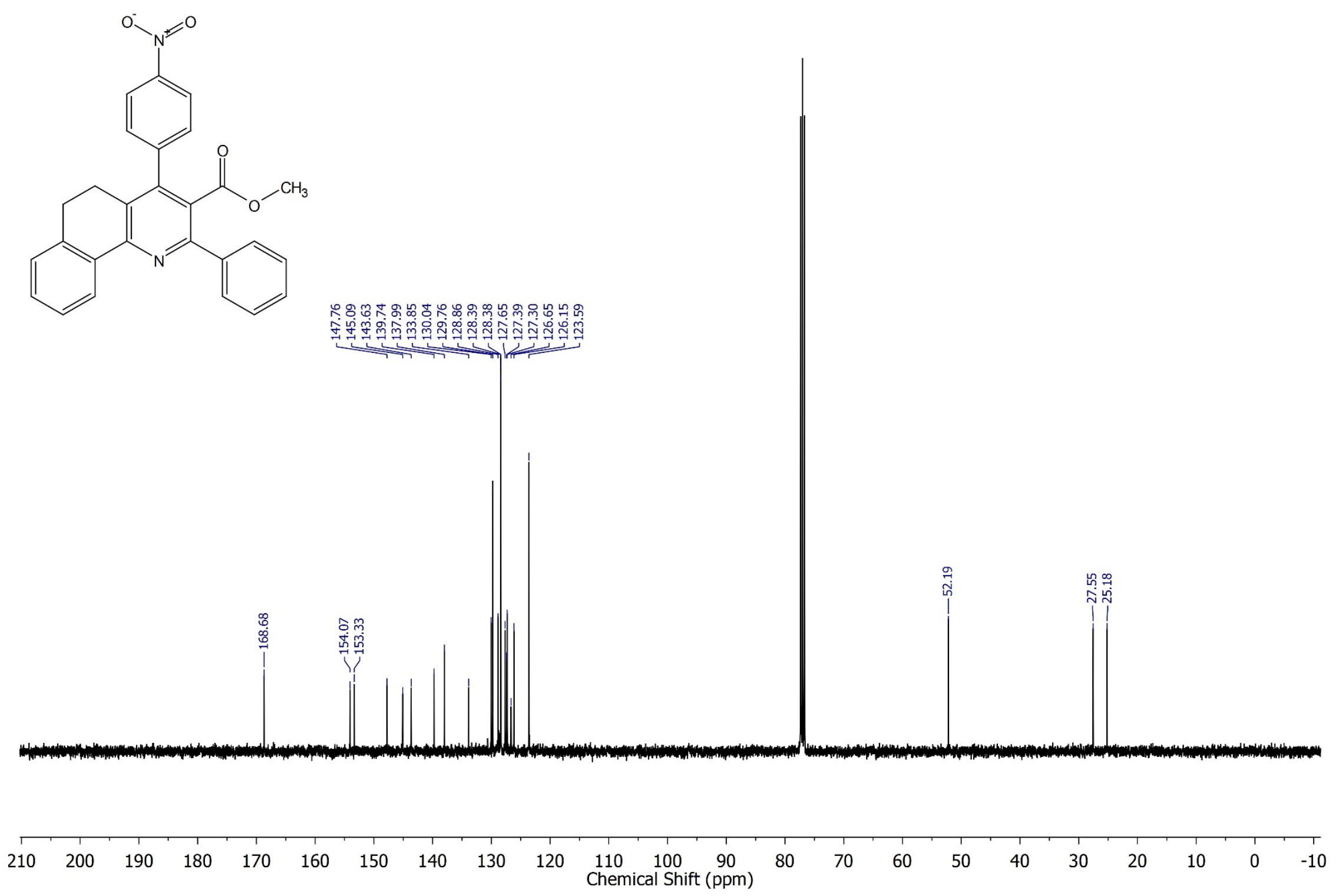


Methyl 4-(4-nitrophenyl)-2-phenyl-5,6-dihydrobenzo[h]quinoline-3-carboxylate 7i, DEPT, $100 \mathrm{MHz}, \mathrm{CDCl}_{3}$
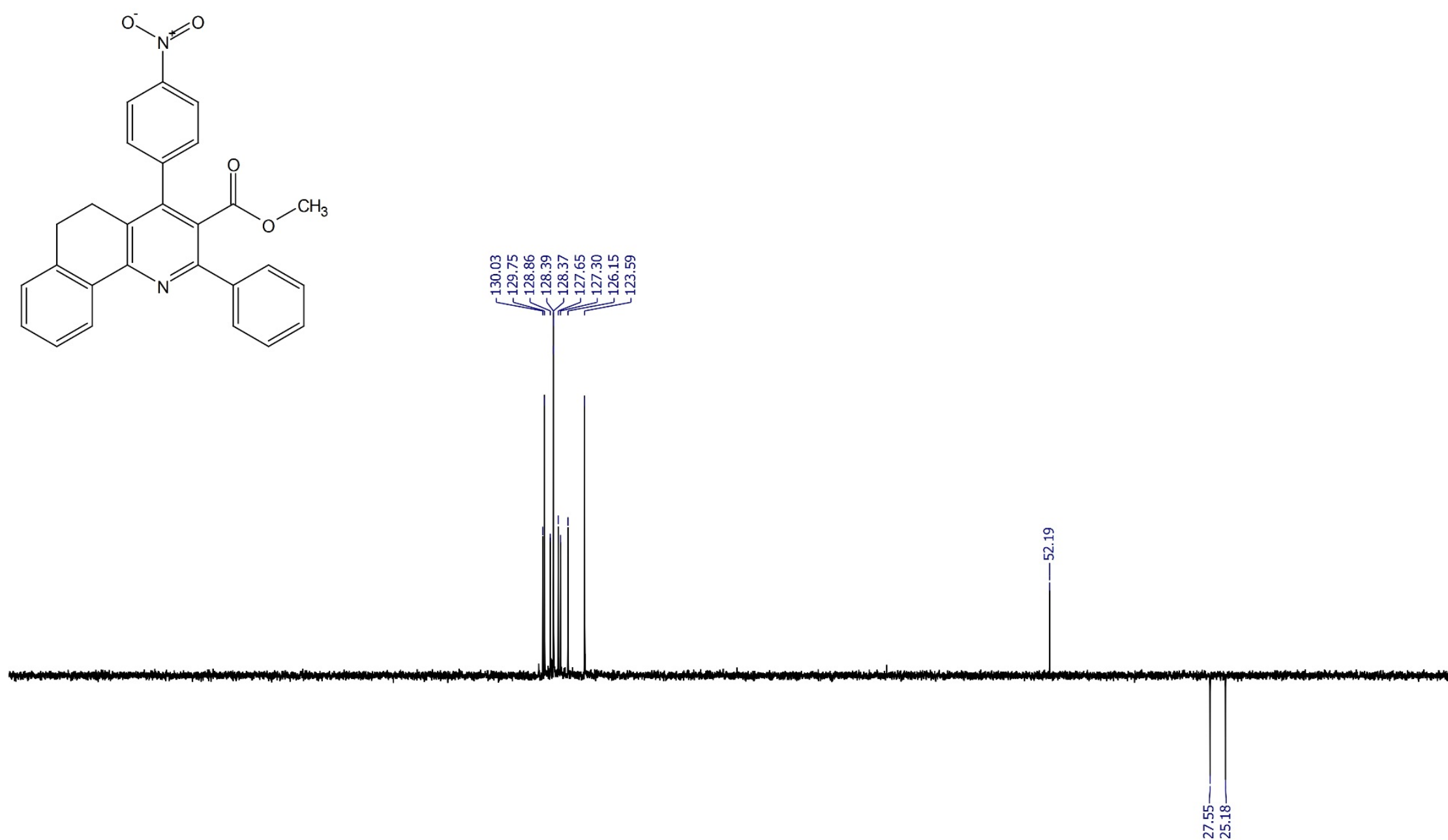
Methyl 4-(4-chlorophenyl)-2-(pyridin-2-yl)-5,6-dihydrobenzo[h]quinoline-3-carboxylate 7j, ${ }^{1} \mathrm{H}$ NMR, $400 \mathrm{MHz} \mathrm{CDCl}_{3}$
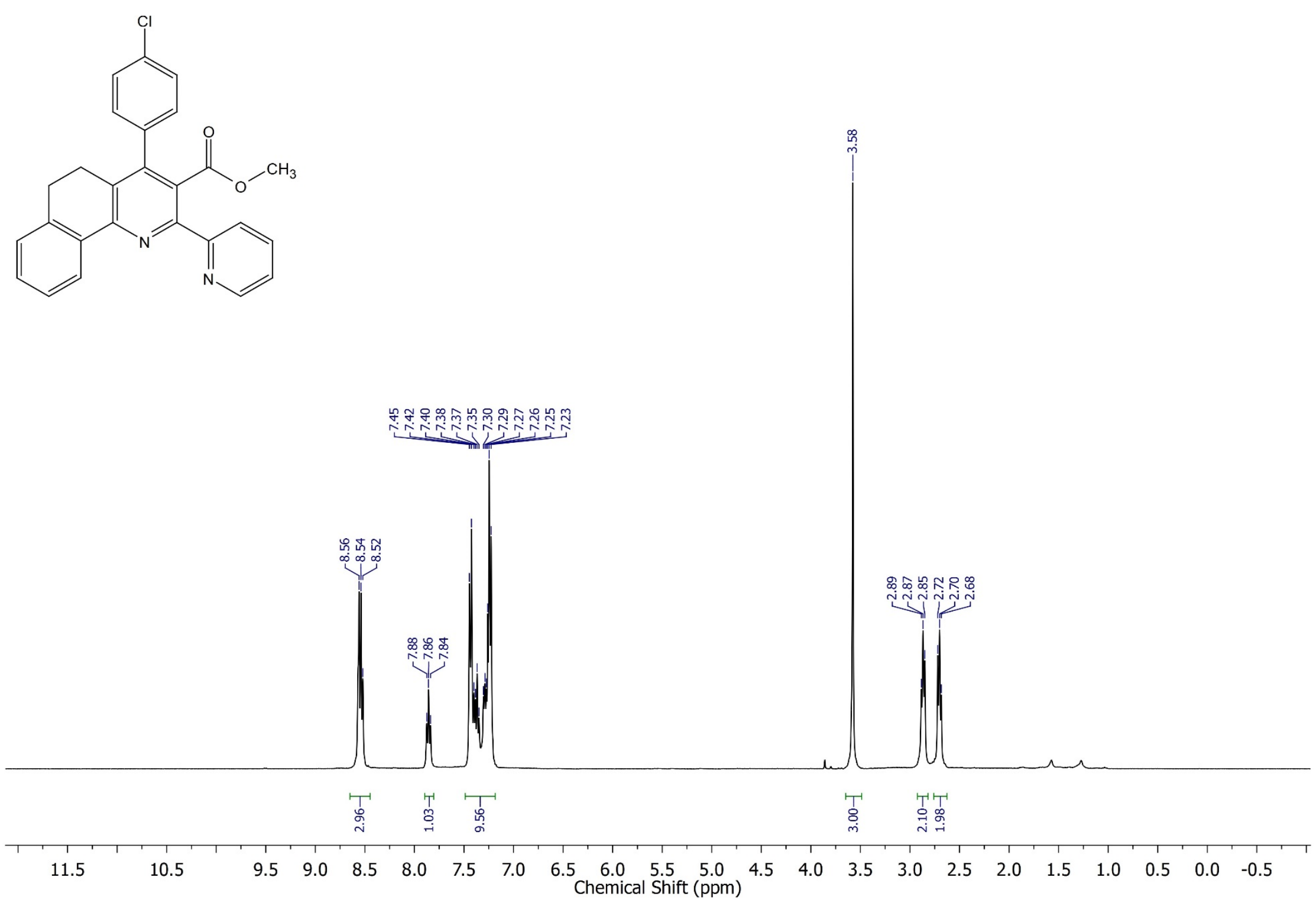
Methyl 4-(4-chlorophenyl)-2-(pyridin-2-yl)-5,6-dihydrobenzo[h]quinoline-3-carboxylate $7 \mathrm{j},{ }^{13} \mathrm{C}\left\{{ }^{1} \mathrm{H}\right\}$ NMR, $100 \mathrm{MHz} \mathrm{CDCl}_{3}$
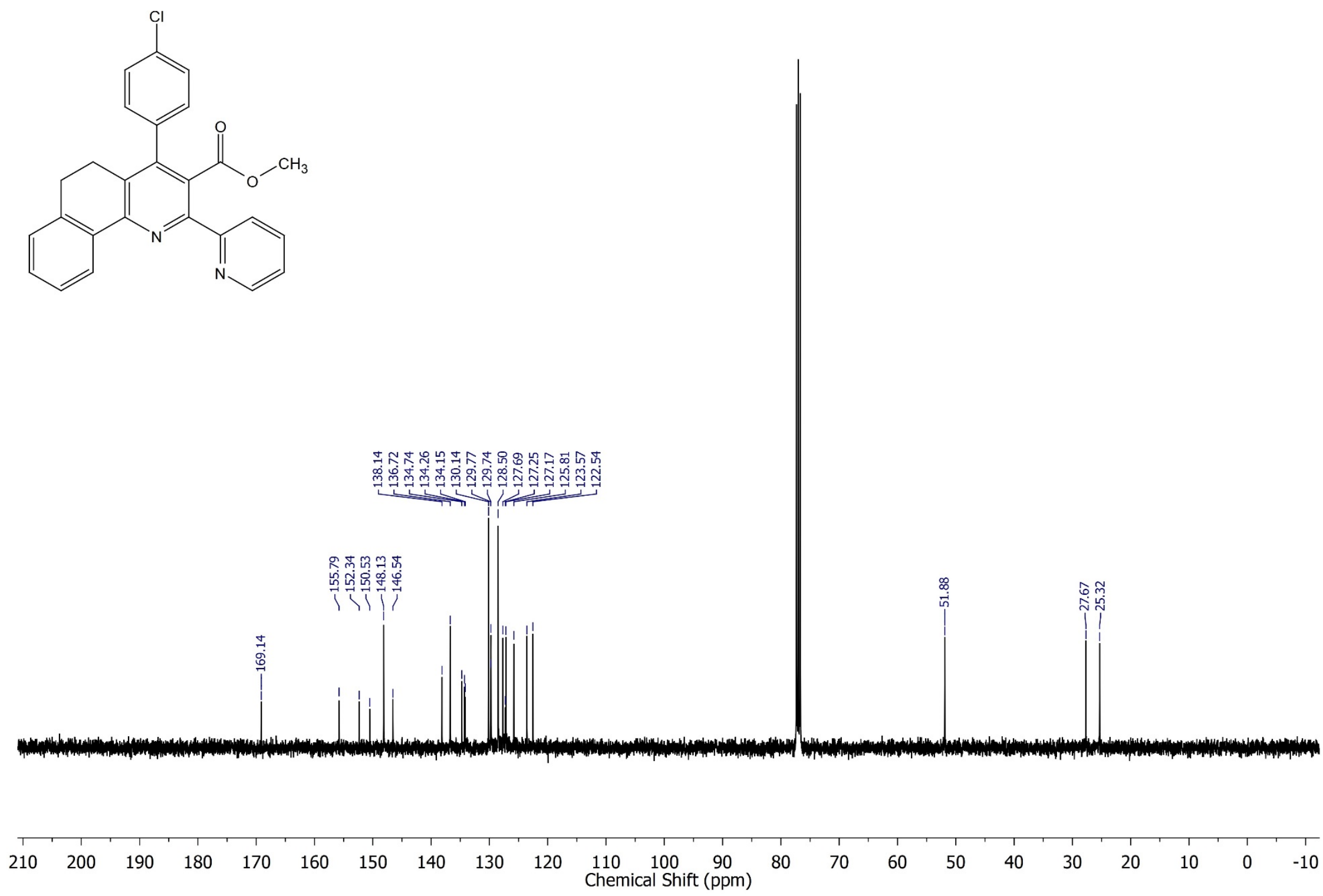
Methyl 4-(4-chlorophenyl)-2-(pyridin-2-yl)-5,6-dihydrobenzo[h]quinoline-3-carboxylate 7j, DEPT, $100 \mathrm{MHz} \mathrm{CDCl}_{3}$

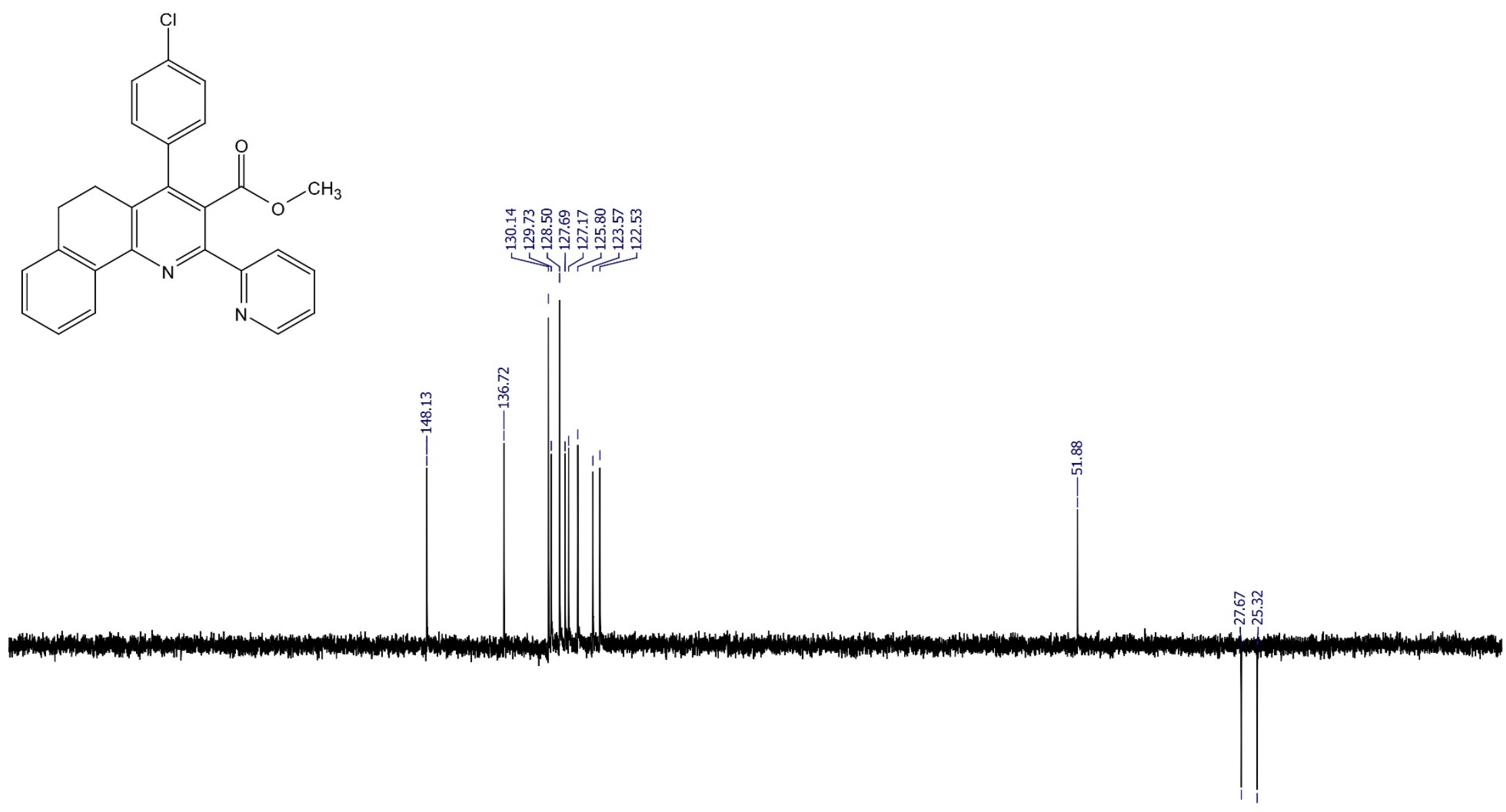

$200 \quad 190$ 
Methyl 2-(quinolin-2-yl)-4-(p-tolyl)-5,6-dihydrobenzo[h]quinoline-3-carboxylate $7 \mathrm{k},{ }^{1} \mathrm{H}$ NMR, $400 \mathrm{MHz}, \mathrm{CDCl}_{3}$

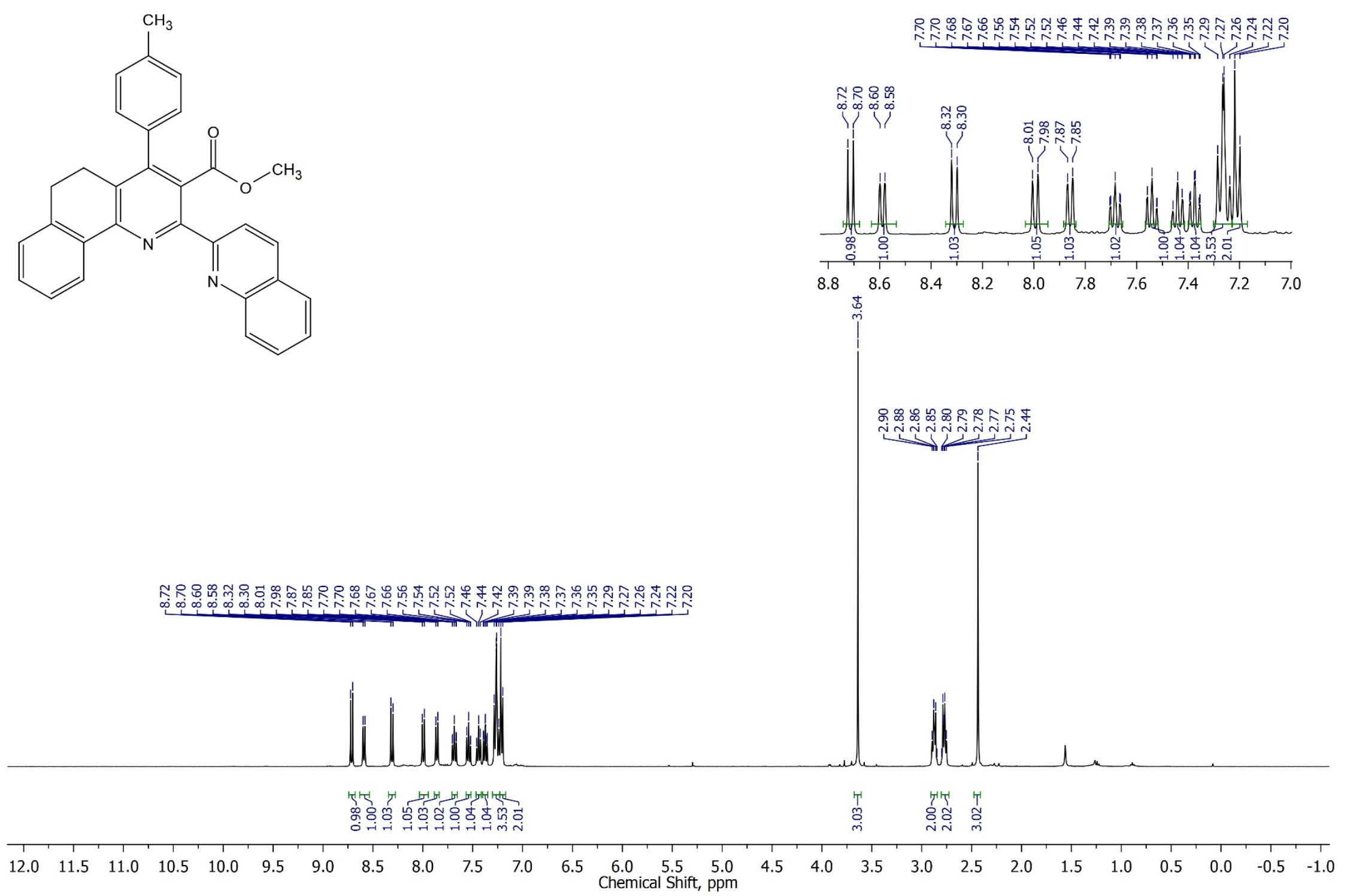


Methyl 2-(quinolin-2-yl)-4-(p-tolyl)-5,6-dihydrobenzo[h]quinoline-3-carboxylate $7 \mathrm{k},{ }^{13} \mathrm{C}\left\{{ }^{1} \mathrm{H}\right\} \mathrm{NMR}, 100 \mathrm{MHz}, \mathrm{CDCl}_{3}$

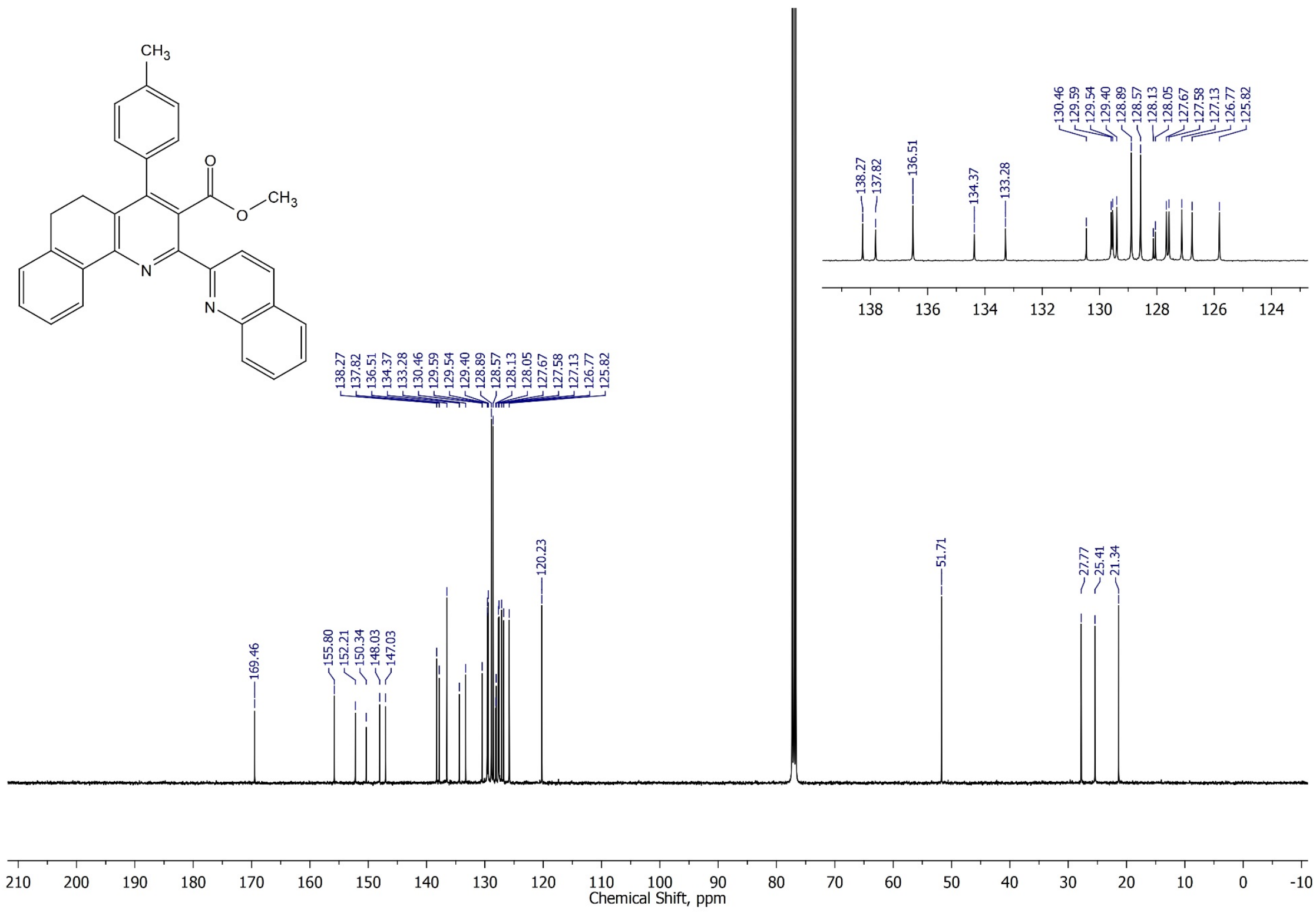


Methyl 2-(quinolin-2-yl)-4-(p-tolyl)-5,6-dihydrobenzo[h]quinoline-3-carboxylate 7k, DEPT, $100 \mathrm{MHz}, \mathrm{CDCl}_{3}$

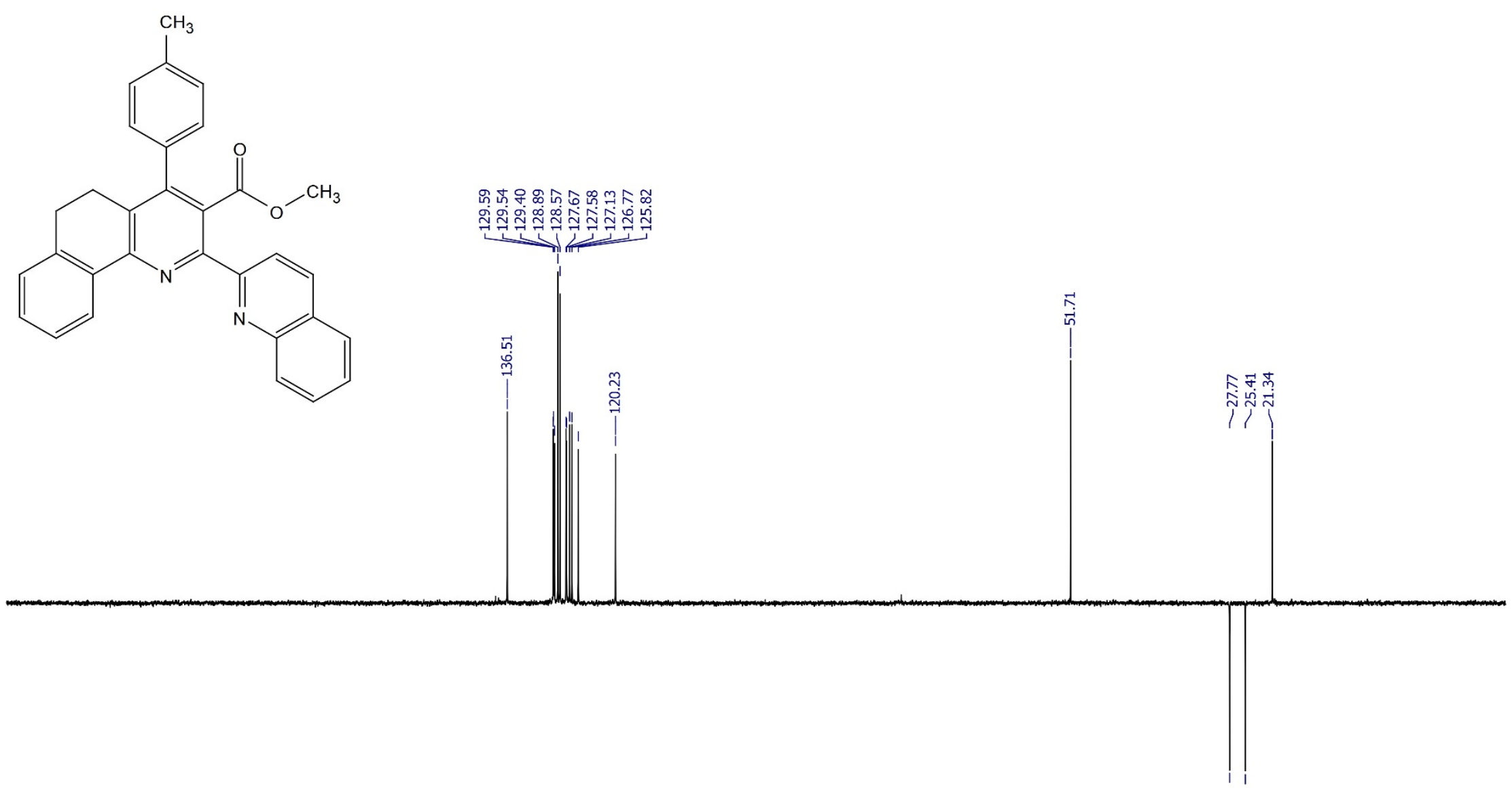

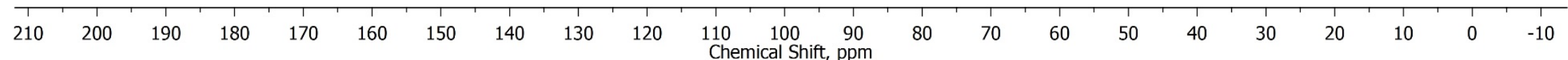


Methyl 4-(3,4-dimethoxyphenyl)-2-(thiophen-2-yl)-5,6-dihydrobenzo[h]quinoline-3-carboxylate $7 \mathrm{l},{ }^{1} \mathrm{H} \mathrm{NMR} \mathrm{NOO}^{\mathrm{MHz}}, \mathrm{CDCl}_{3}$

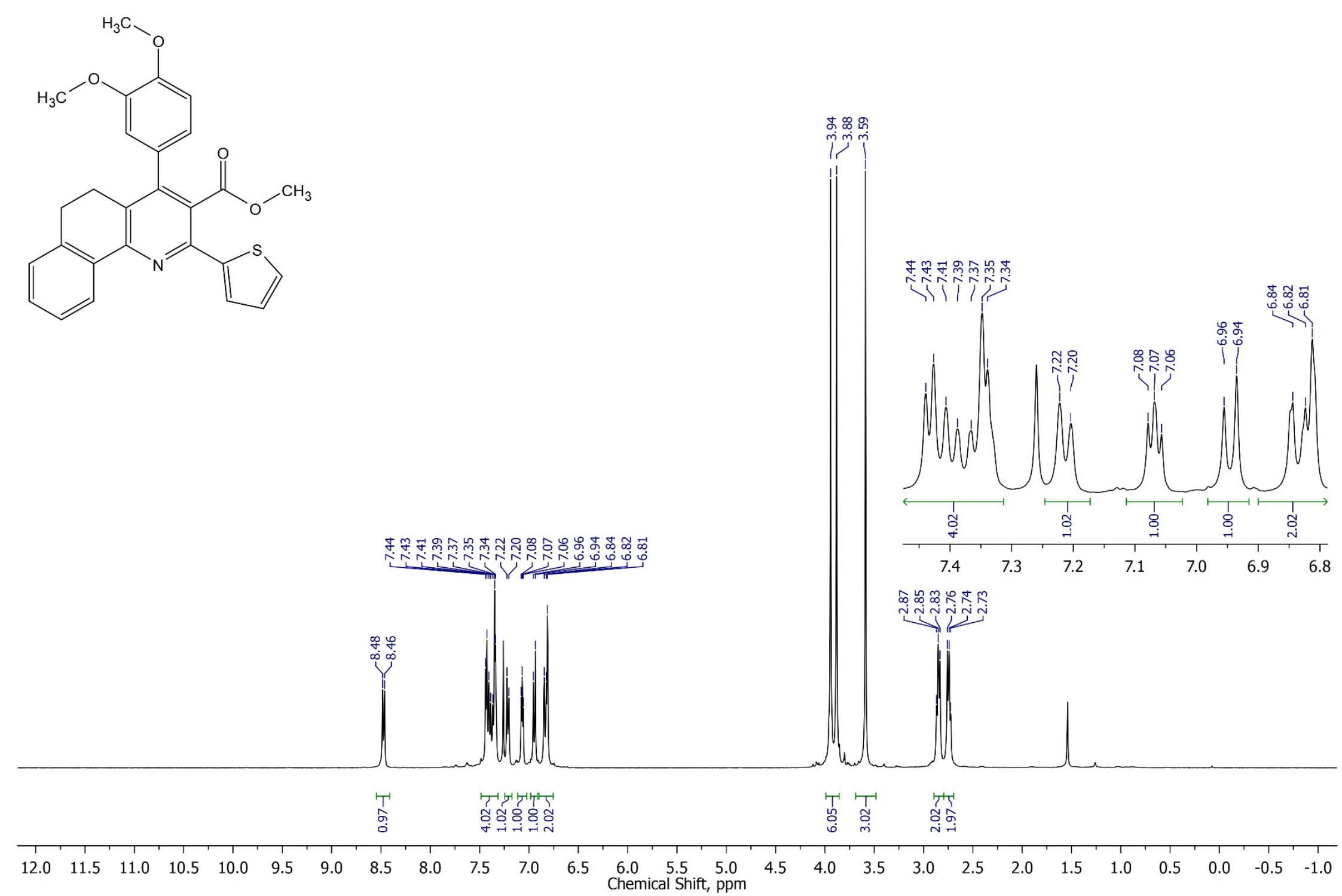


Methyl 4-(3,4-dimethoxyphenyl)-2-(thiophen-2-yl)-5,6-dihydrobenzo[h]quinoline-3-carboxylate $7 \mathrm{l},{ }^{13} \mathrm{C}\left\{{ }^{1} \mathrm{H}\right\} \mathrm{NMR}, 100 \mathrm{MHz}, \mathrm{CDCl}_{3}$
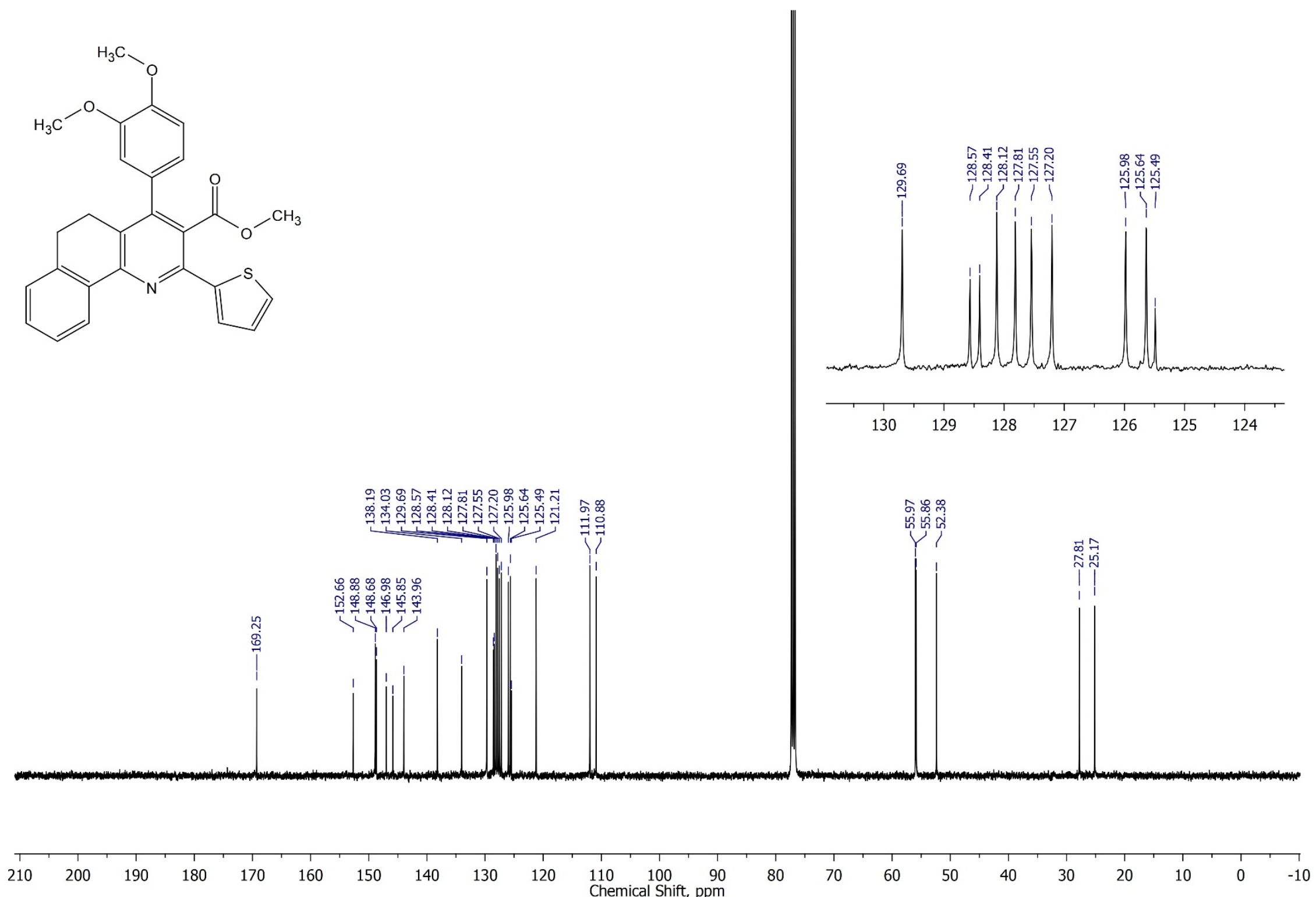
Methyl 4-(3,4-dimethoxyphenyl)-2-(thiophen-2-yl)-5,6-dihydrobenzo[h]quinoline-3-carboxylate 7l, DEPT, $100 \mathrm{MHz}^{\mathrm{CDCl}} \mathrm{CD}_{3}$
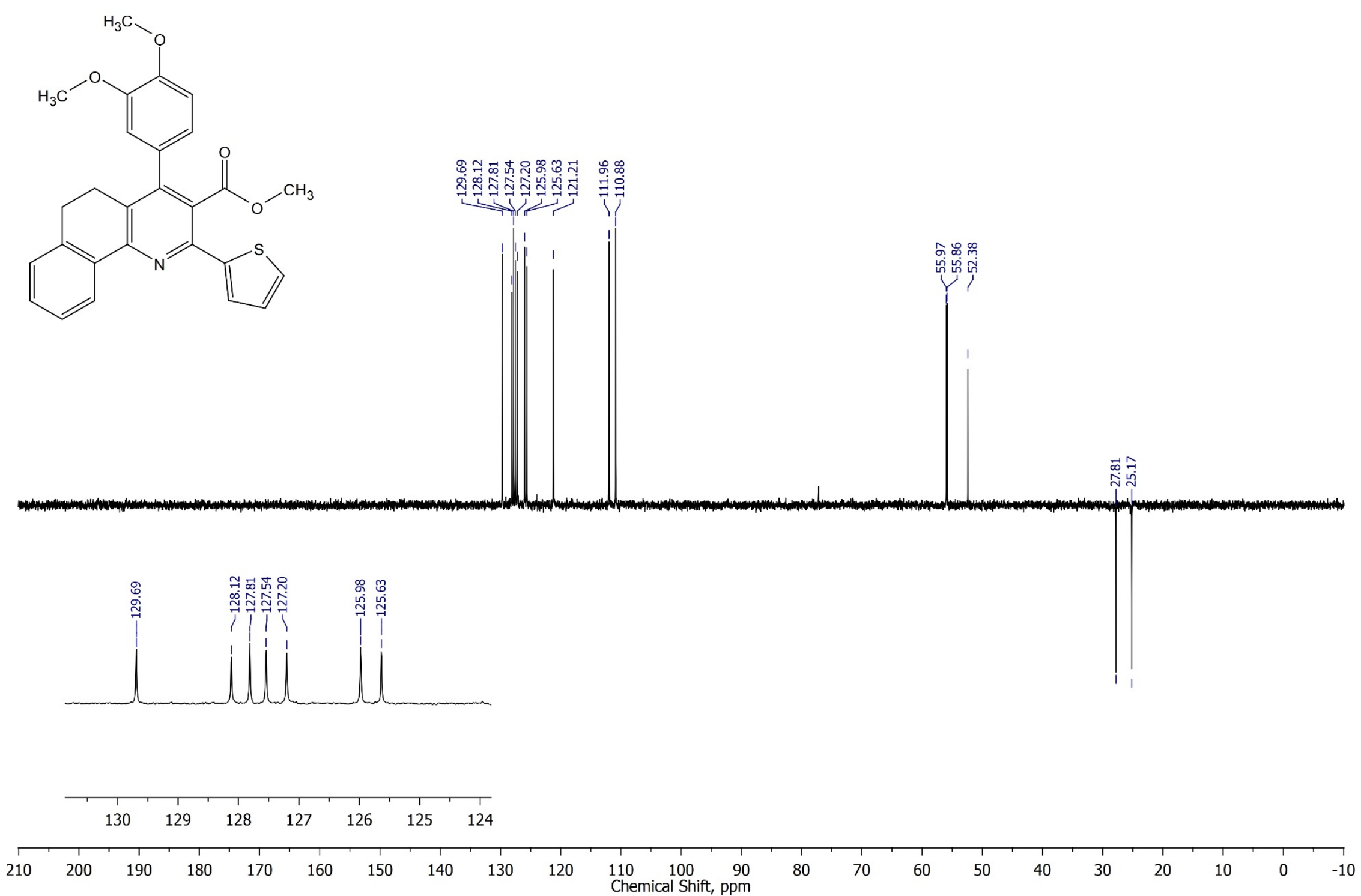
Methyl 2-(4-bromophenyl)-4-cyclopropyl-9-methoxy-5,6-dihydrobenzo[h]quinoline-3-carboxylate $7 \mathrm{~m},{ }^{1} \mathrm{H} \mathrm{NMR}, 400 \mathrm{MHz}, \mathrm{CDCl}_{3}$
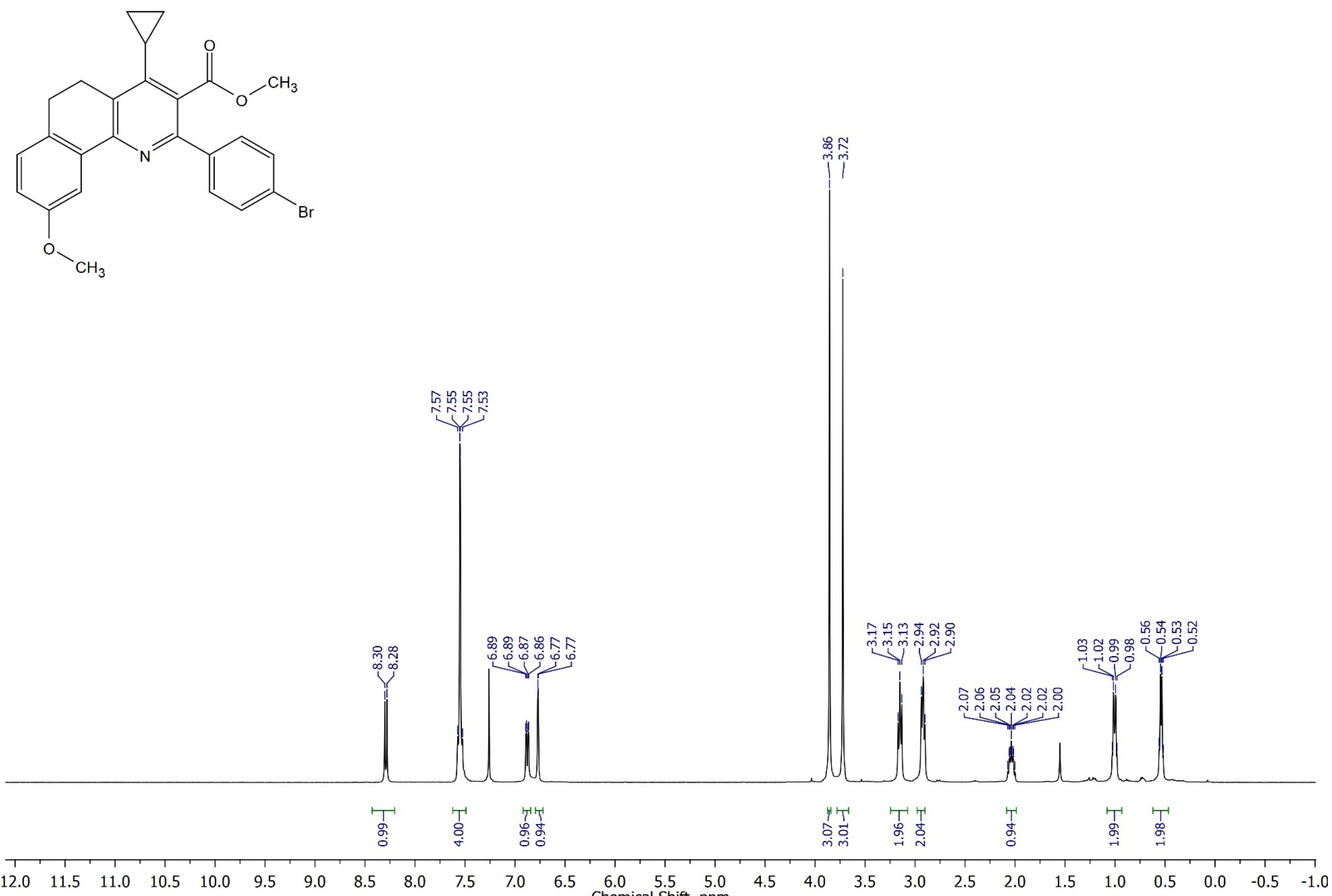
Methyl 2-(4-bromophenyl)-4-cyclopropyl-9-methoxy-5,6-dihydrobenzo[h]quinoline-3-carboxylate $7 \mathrm{~m},{ }^{13} \mathrm{C}\left\{{ }^{1} \mathrm{H}\right\} \mathrm{NMR}^{100} \mathrm{MHz}, \mathrm{CDCl}{ }_{3}$
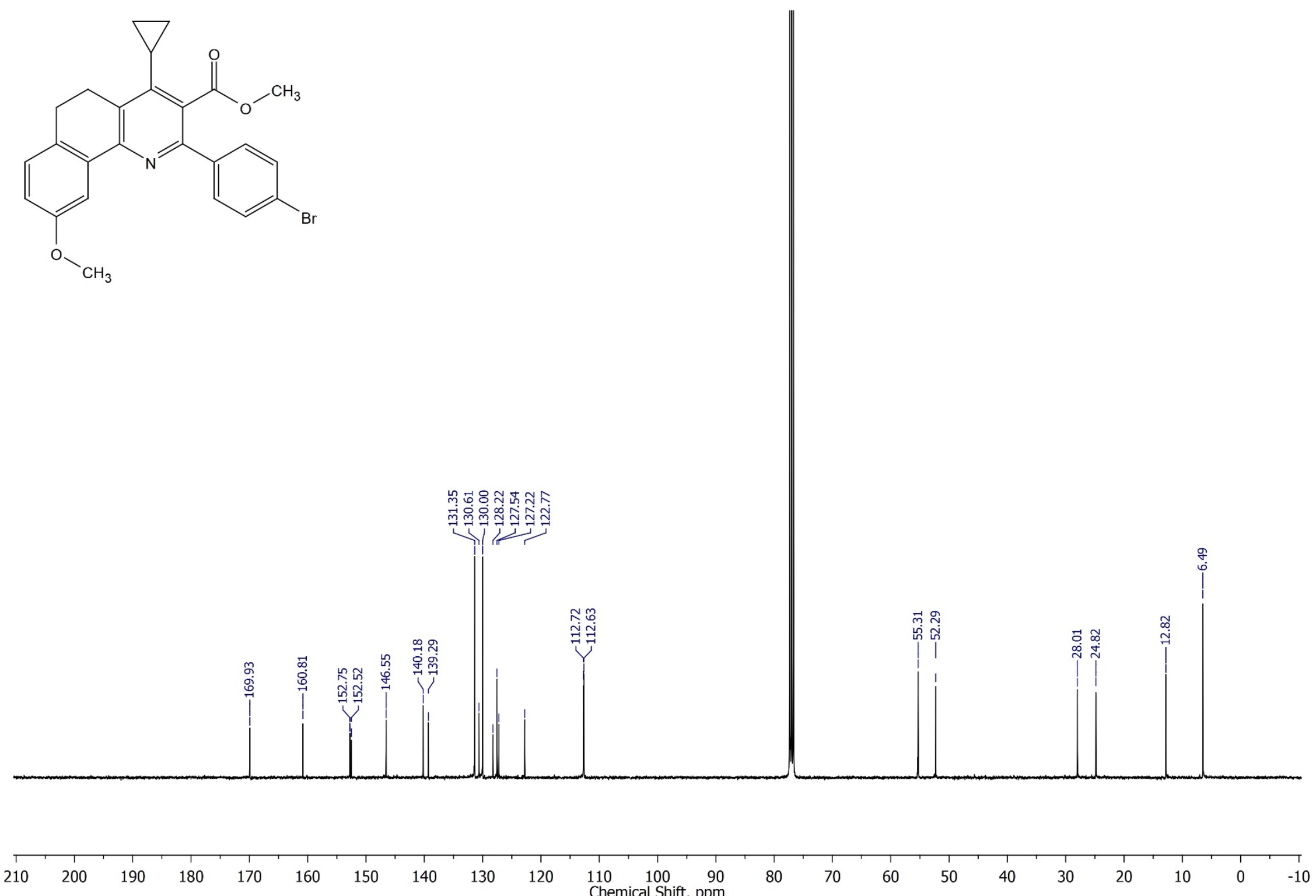
Methyl 2-(4-bromophenyl)-4-cyclopropyl-9-methoxy-5,6-dihydrobenzo[h]quinoline-3-carboxylate 7m, DEPT, $100 \mathrm{MHz}^{\mathrm{m}} \mathrm{CDCl}_{3}$
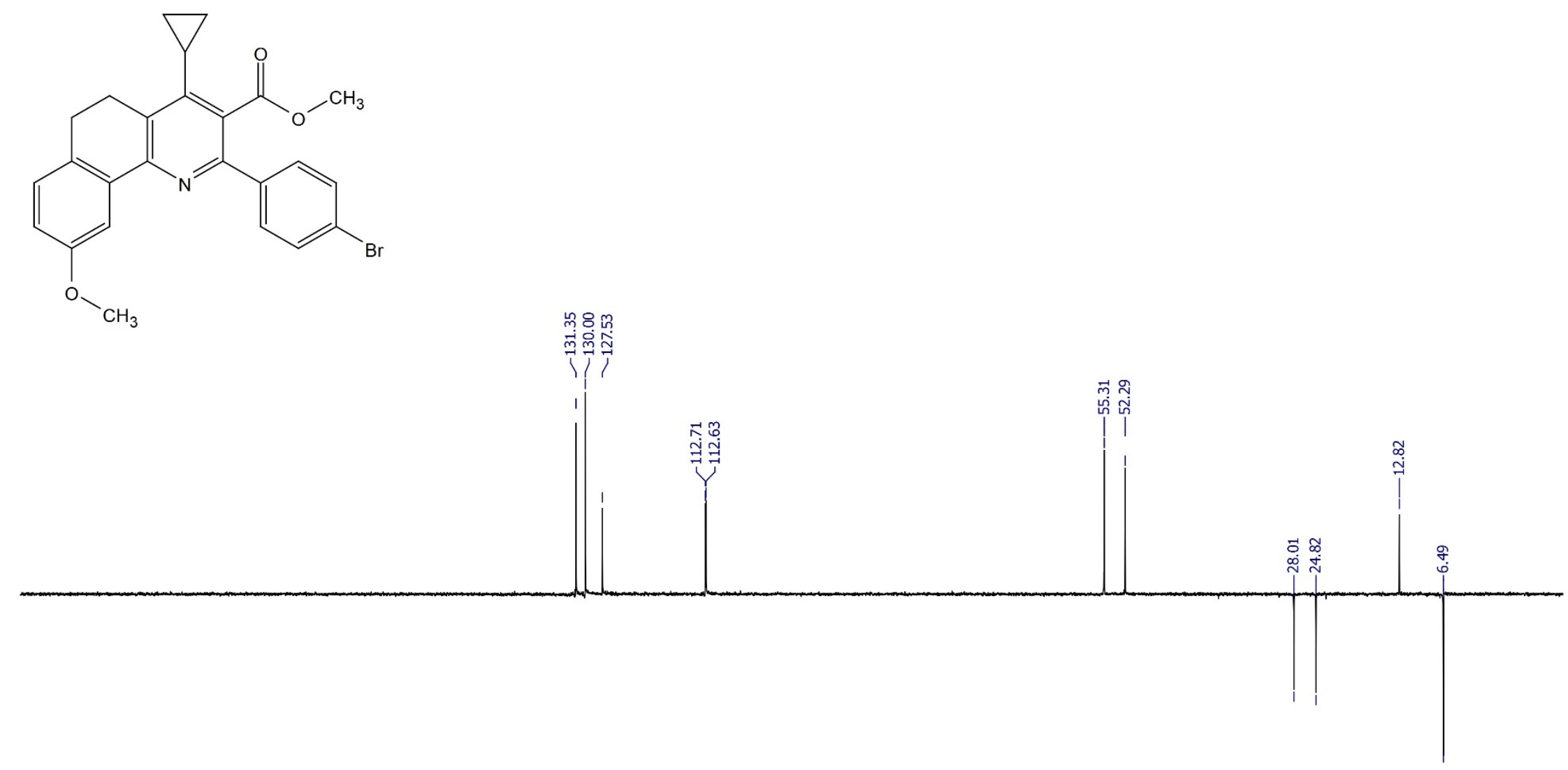

$\begin{array}{rrrrrrrrrrrrrrrrrrrrrrrrr}1 & 210 & 200 & 190 & 180 & 170 & 160 & 150 & 140 & 130 & 120 & 110 & 100 & 90 & 80 & 70 & 60 & 50 & 40 & 30 & 20 & 10 & 0 & -10\end{array}$


Methyl 2-(tert-butyl)-4-(4-(tert-butyl)phenyl)-9-methoxy-5,6-dihydrobenzo[h]quinoline-3-carboxylate $7 \mathrm{n},{ }^{1} \mathrm{H} \mathrm{NMR,}^{400} \mathrm{MHz} \mathrm{CDCl}_{3}$
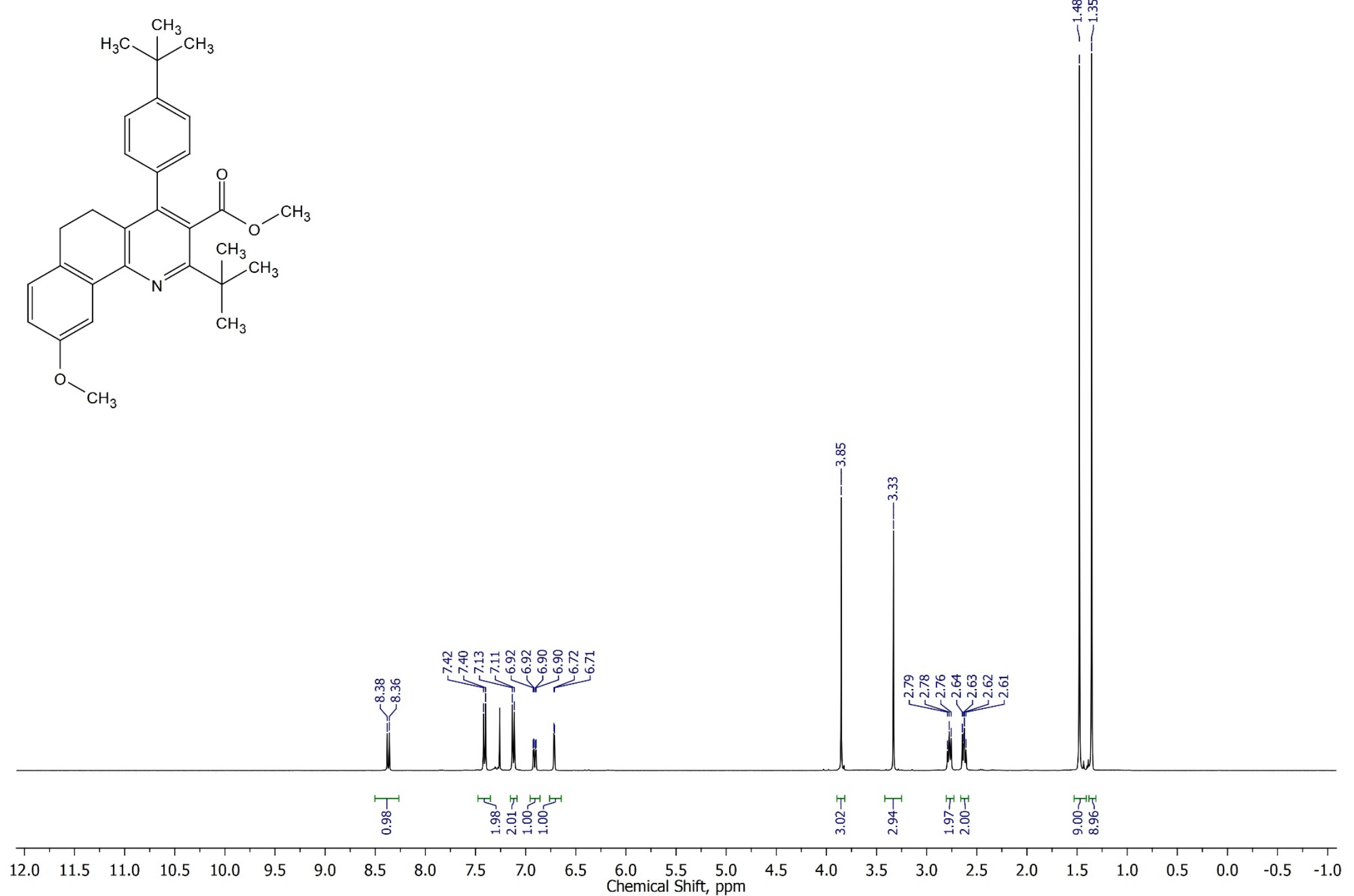
Methyl 2-(tert-butyl)-4-(4-(tert-butyl)phenyl)-9-methoxy-5,6-dihydrobenzo[h]quinoline-3-carboxylate $7 \mathrm{n},{ }^{13} \mathrm{C}\left\{{ }^{1} \mathrm{H}\right\}$ NMR, $100 \mathrm{MHz}, \mathrm{CDCl}_{3}$
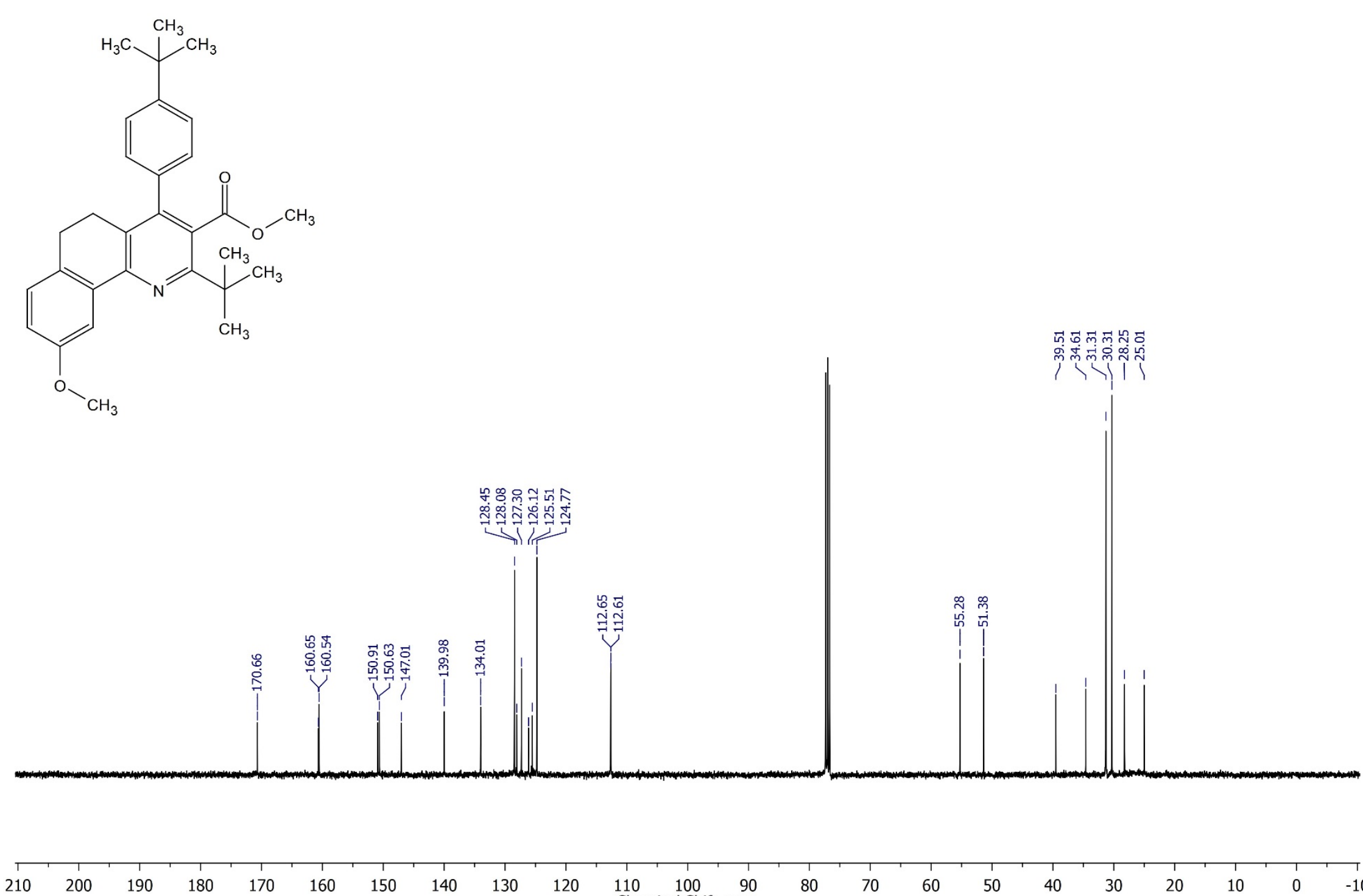

180

$170 \quad 160$

150

$140 \quad 130$

120

$\begin{array}{lll}110 & 100 & 90\end{array}$

80

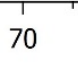

60

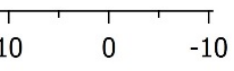


Methyl 2-(tert-butyl)-4-(4-(tert-butyl)phenyl)-9-methoxy-5,6-dihydrobenzo[h]quinoline-3-carboxylate 7n, DEPT, $100 \mathrm{MHz}^{\mathrm{C}} \mathrm{CDCl}{ }_{3}$

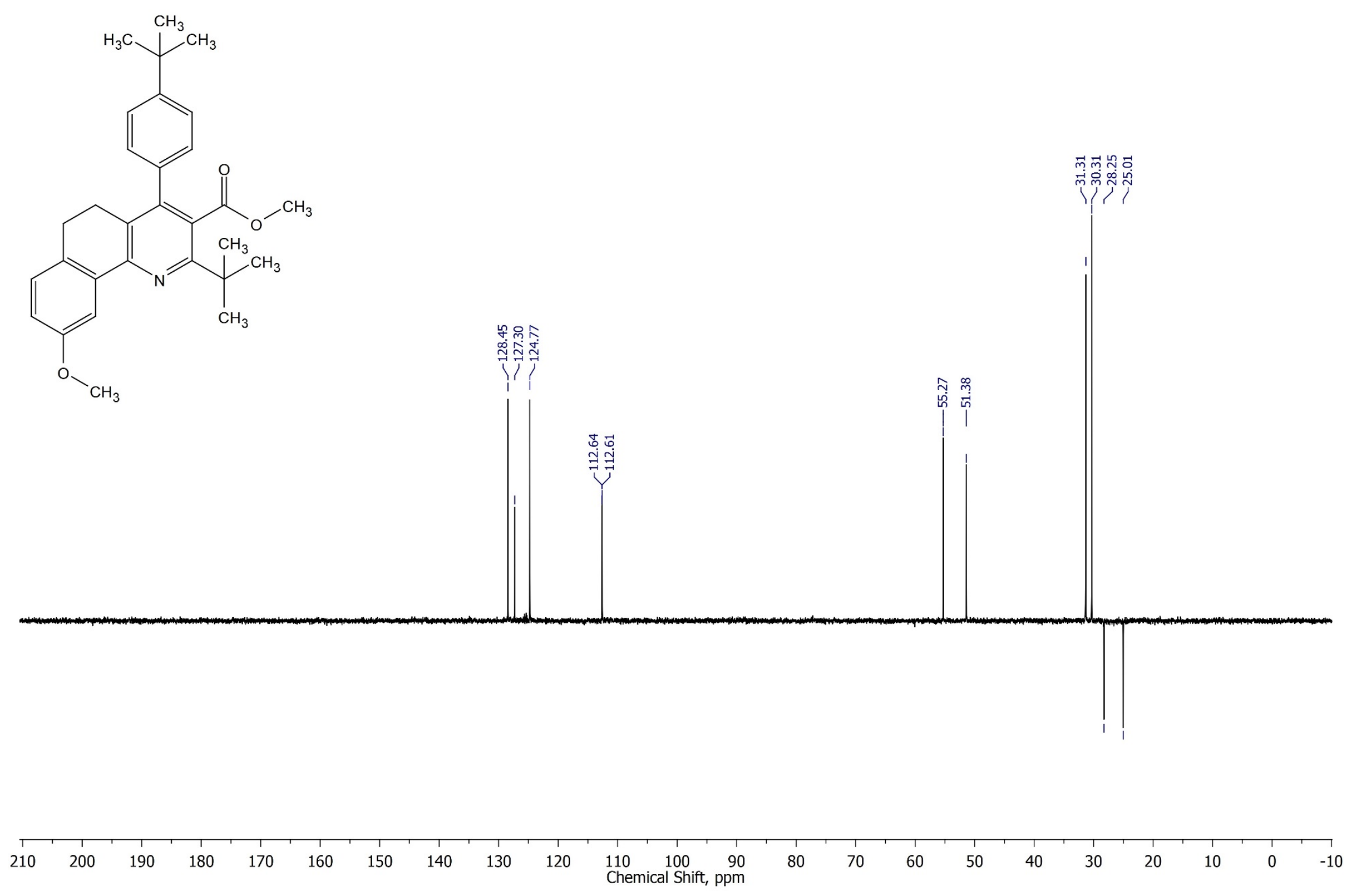


Methyl 4-(4-bromophenyl)-5-methyl-2,6-diphenylnicotinate $7 \mathrm{~m},{ }^{1} \mathrm{H} \mathrm{NMR}, 400 \mathrm{MHz}, \mathrm{CDCl}_{3}$
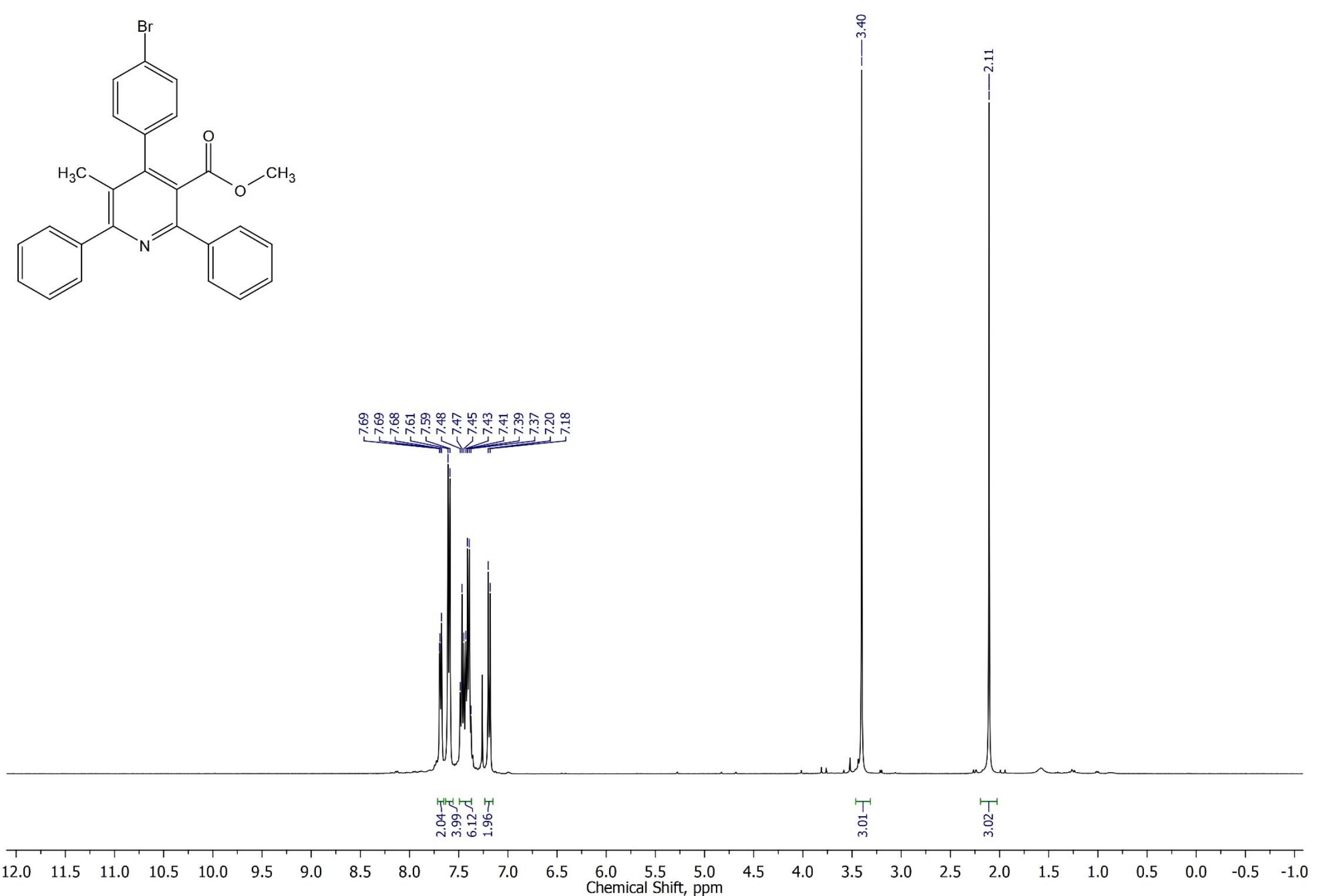
Methyl 4-(4-bromophenyl)-5-methyl-2,6-diphenylnicotinate $7 \mathrm{~m},{ }^{13} \mathrm{C}\left\{{ }^{1} \mathrm{H}\right\} \mathrm{NMR}, 100 \mathrm{MHz}, \mathrm{CDCl}_{3}$

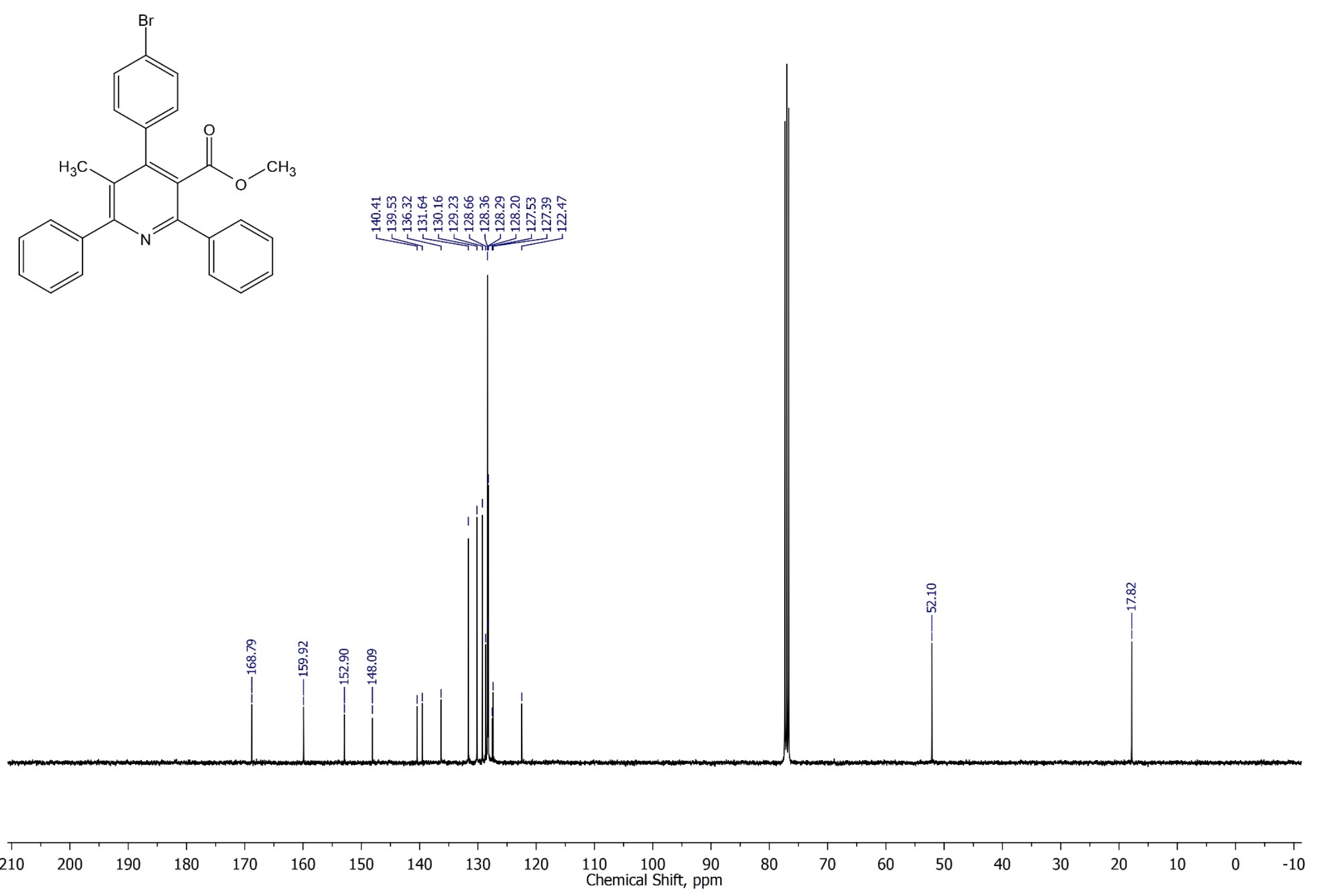


Methyl 4-(4-bromophenyl)-5-methyl-2,6-diphenylnicotinate 7m, DEPT, $100 \mathrm{MHz}, \mathrm{CDCl}_{3}$
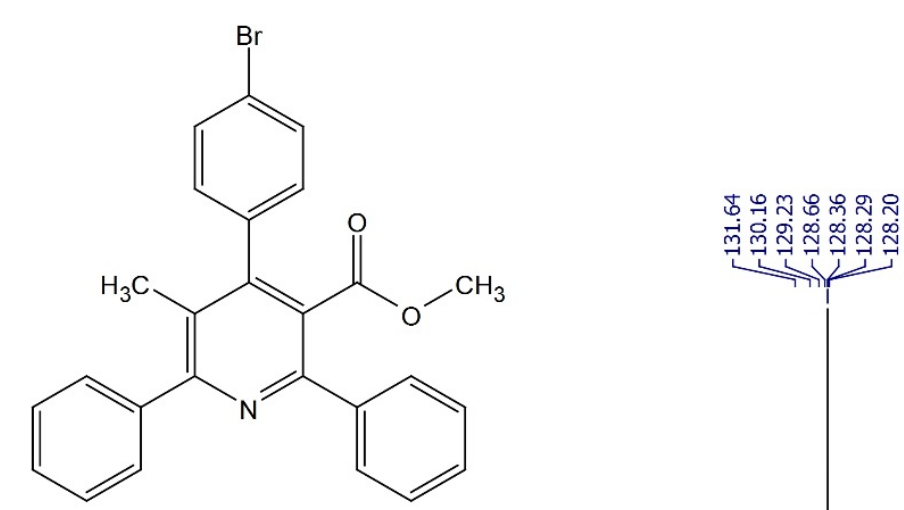

210

$200 \quad 190$

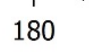

$170 \quad 160$

150

140

$130 \quad 120$

$\begin{array}{cc}110 & 100 \\ \text { Chemical Shift, } & 90 \\ \text { ppm }\end{array}$ 
4-(4-Bromophenyl)-2-phenyl-5,6,7,8-tetrahydroquinoline $8,{ }^{1} \mathrm{H} \mathrm{NMR}, 400 \mathrm{MHz}, \mathrm{CDCl}_{3}$
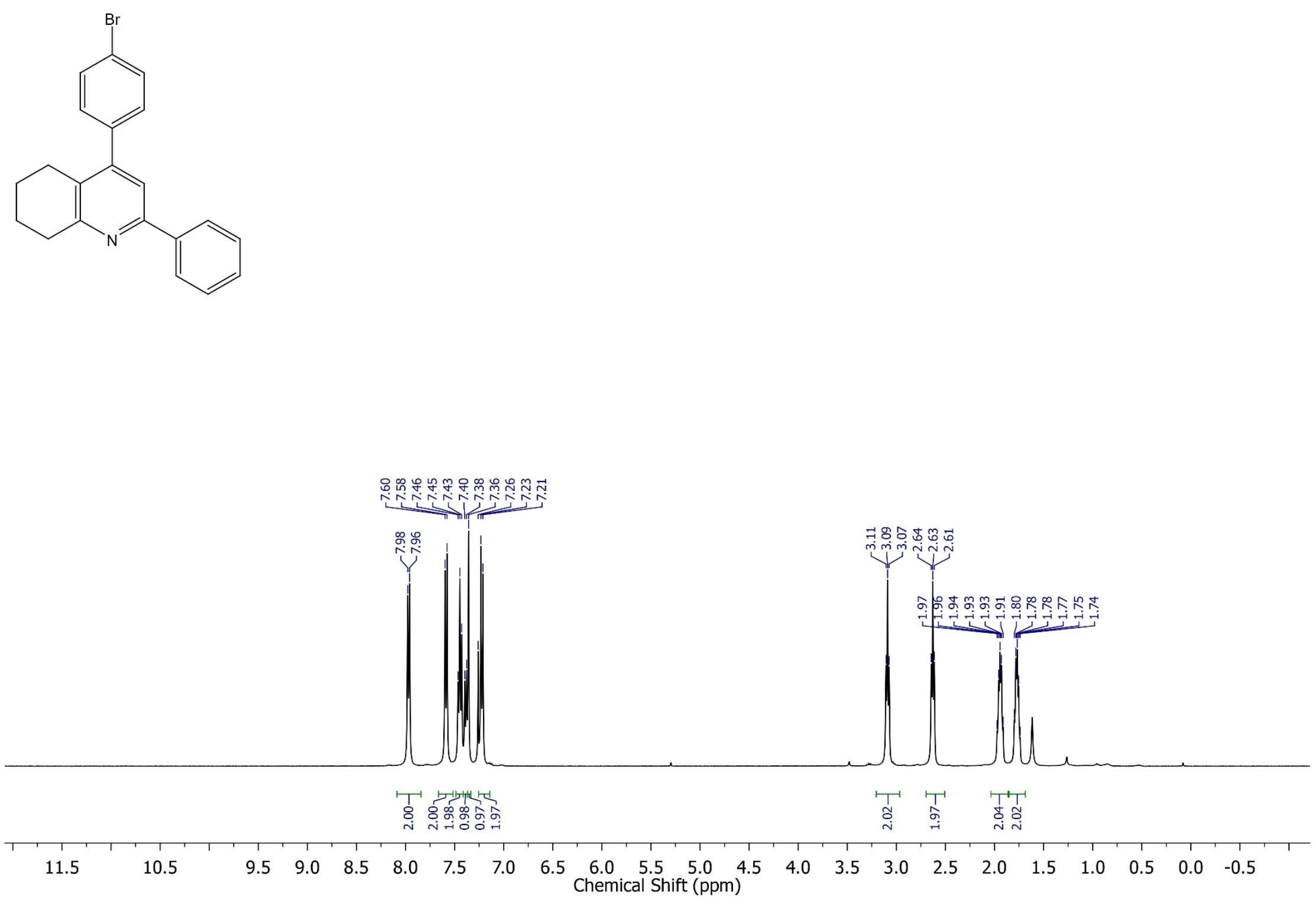
4-(4-Bromophenyl)-2-phenyl-5,6,7,8-tetrahydroquinoline $8,{ }^{13} \mathrm{C}\left\{{ }^{1} \mathrm{H}\right\} \mathrm{NMR}, 100 \mathrm{MHz}, \mathrm{CDCl}_{3}$
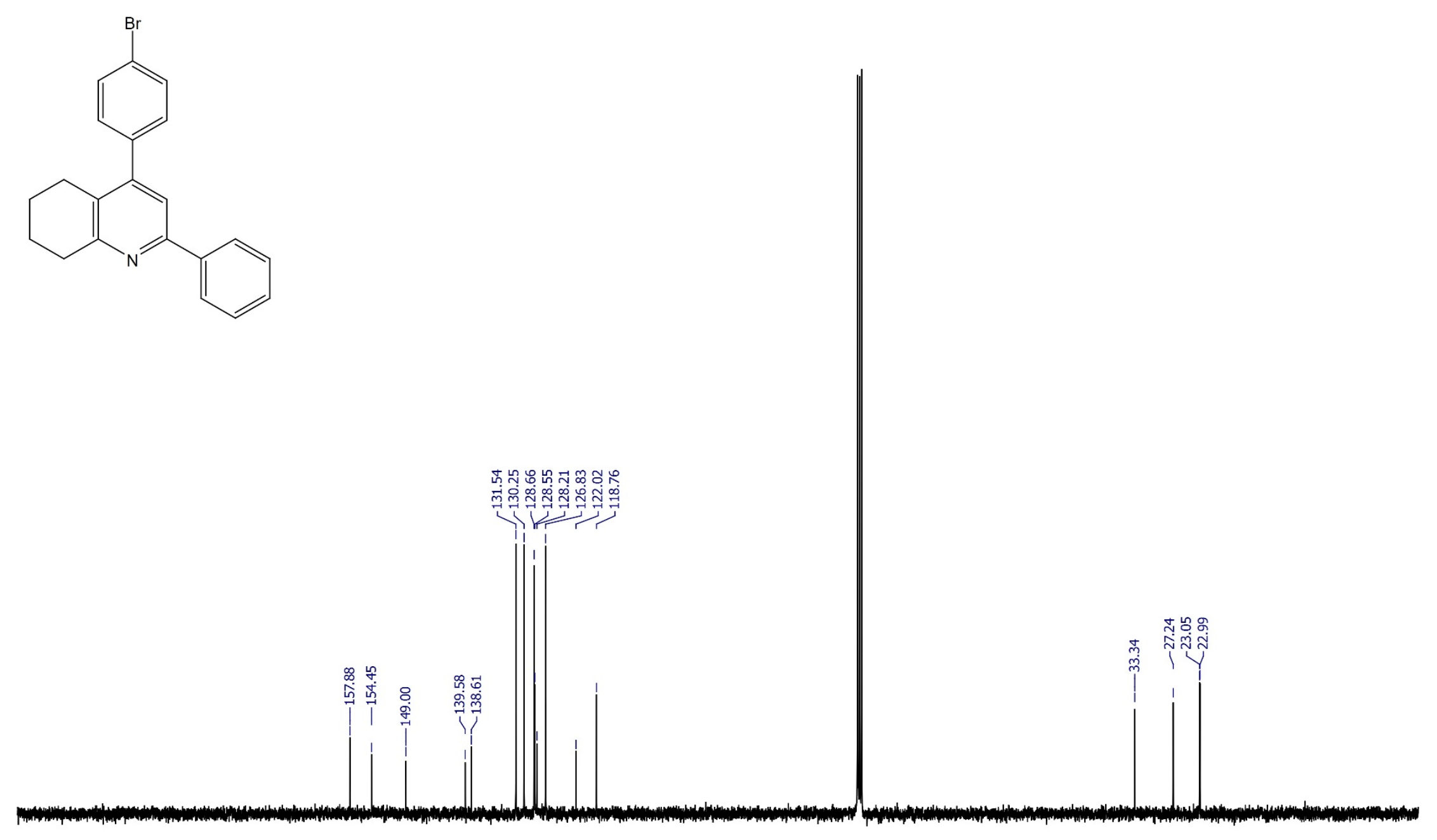

210

200

$190 \quad 180$

170

160150

140

130

120

$\begin{array}{lll}110 & 100 & 90 \\ \text { Chemical Shift } & (\mathrm{ppm})\end{array}$

$80 \quad 70$

60

50

$40 \quad 30$

20

$10 \quad 0$

$-10$ 
4-(4-Bromophenyl)-2-phenyl-5,6,7,8-tetrahydroquinoline 8, DEPT, $100 \mathrm{MHz}, \mathrm{CDCl}_{3}$

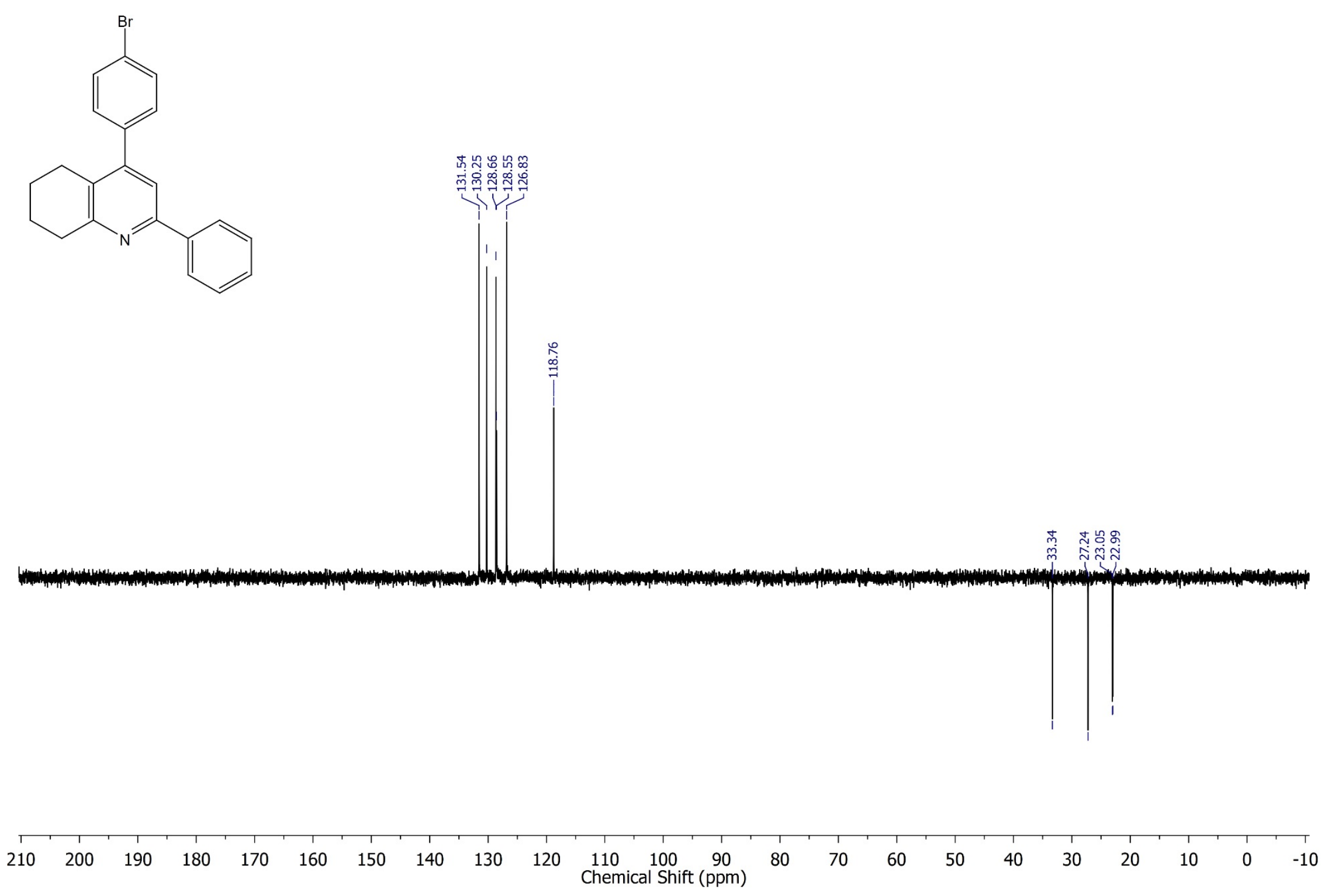


Methyl 4-(4-methoxyphenyl)-2-phenylquinoline-3-carboxylate 9, ${ }^{1} \mathrm{H}$ NMR, $400 \mathrm{MHz}, \mathrm{CDCl}_{3}$
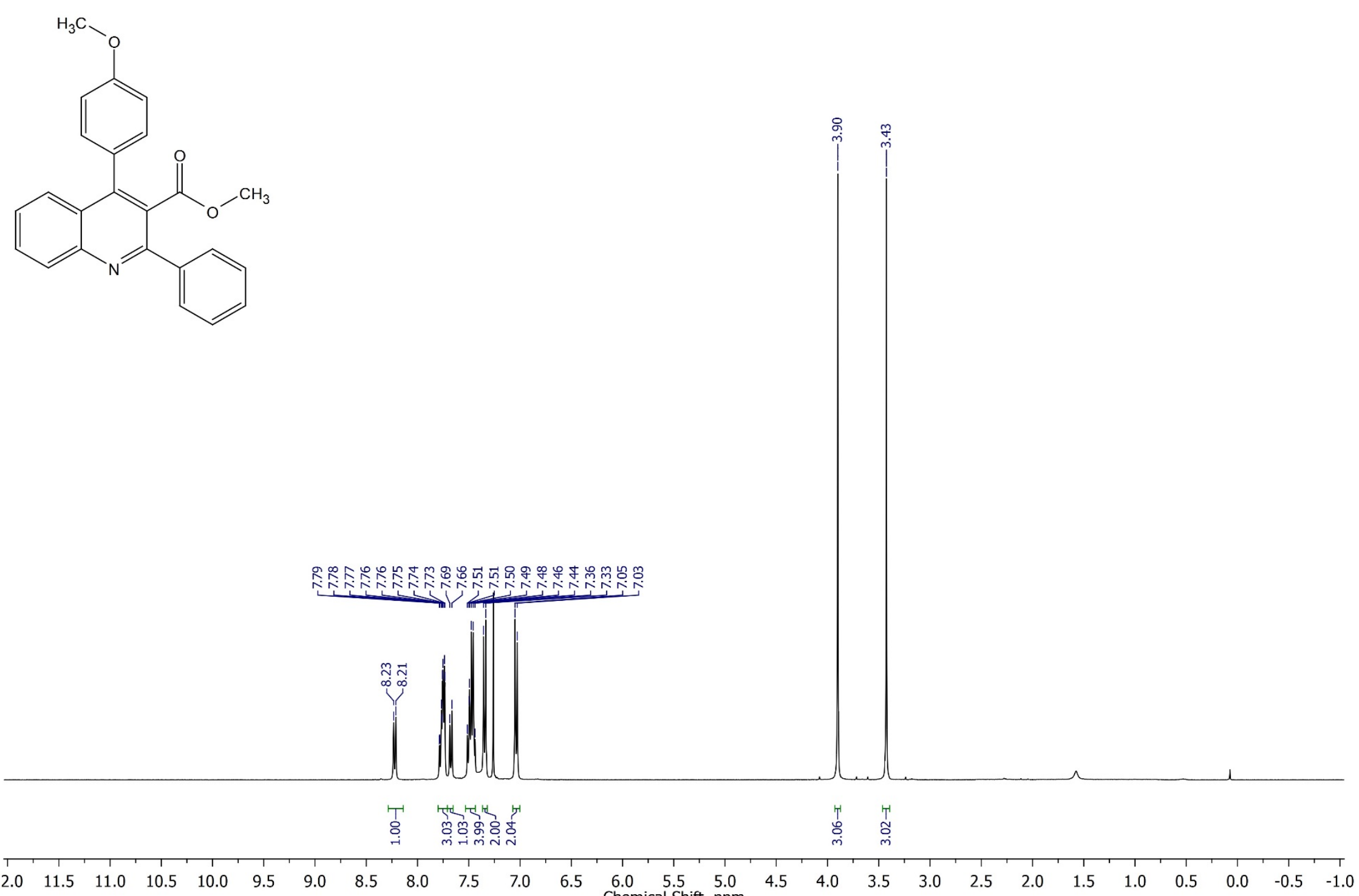
Methyl 4-(4-methoxyphenyl)-2-phenylquinoline-3-carboxylate $9,{ }^{13} \mathrm{C}\left\{{ }^{1} \mathrm{H}\right\} \mathrm{NMR}, 100 \mathrm{MHz}, \mathrm{CDCl}_{3}$
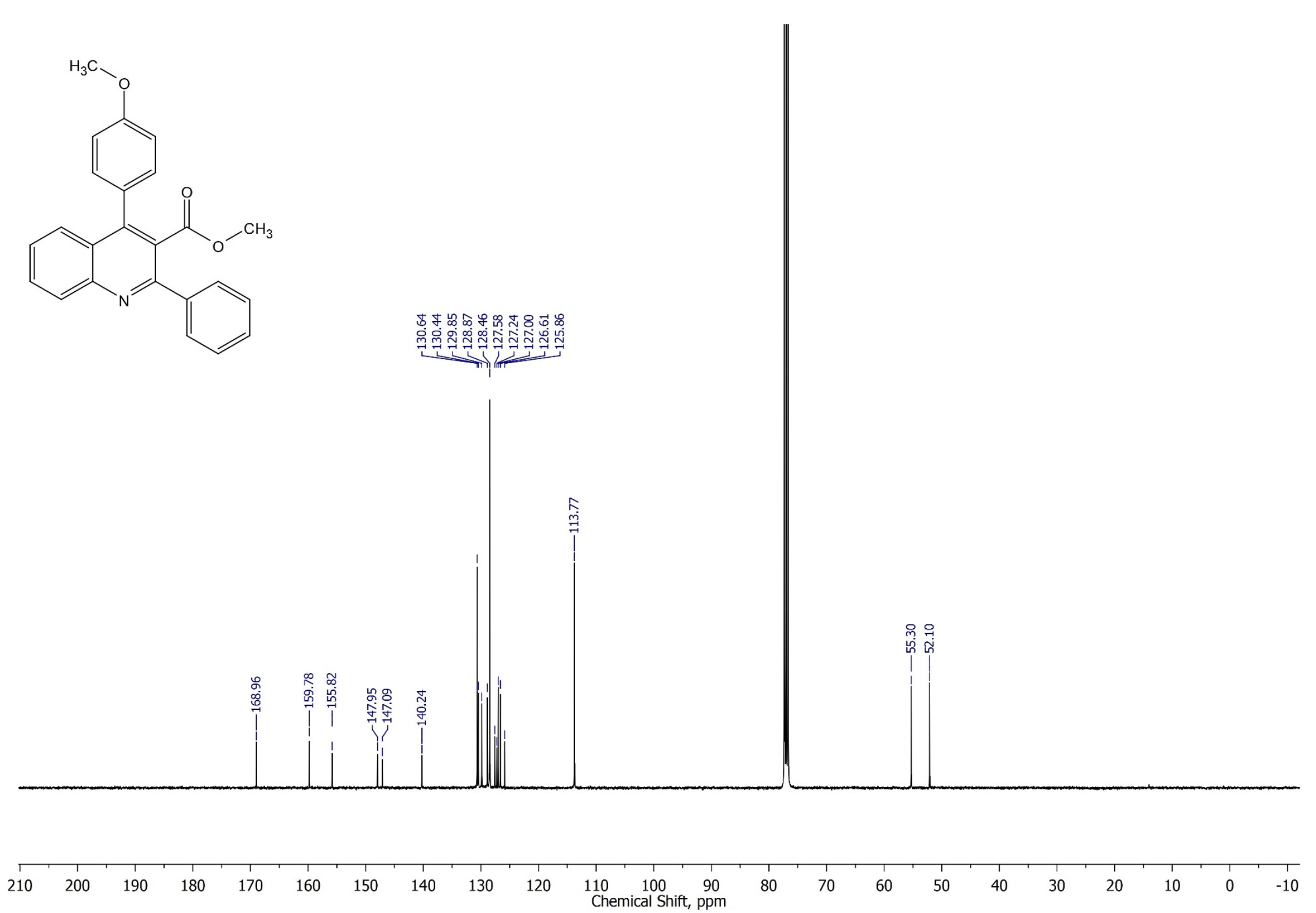
Methyl 4-(4-methoxyphenyl)-2-phenylquinoline-3-carboxylate 9, DEPT, $100 \mathrm{MHz}, \mathrm{CDCl}_{3}$
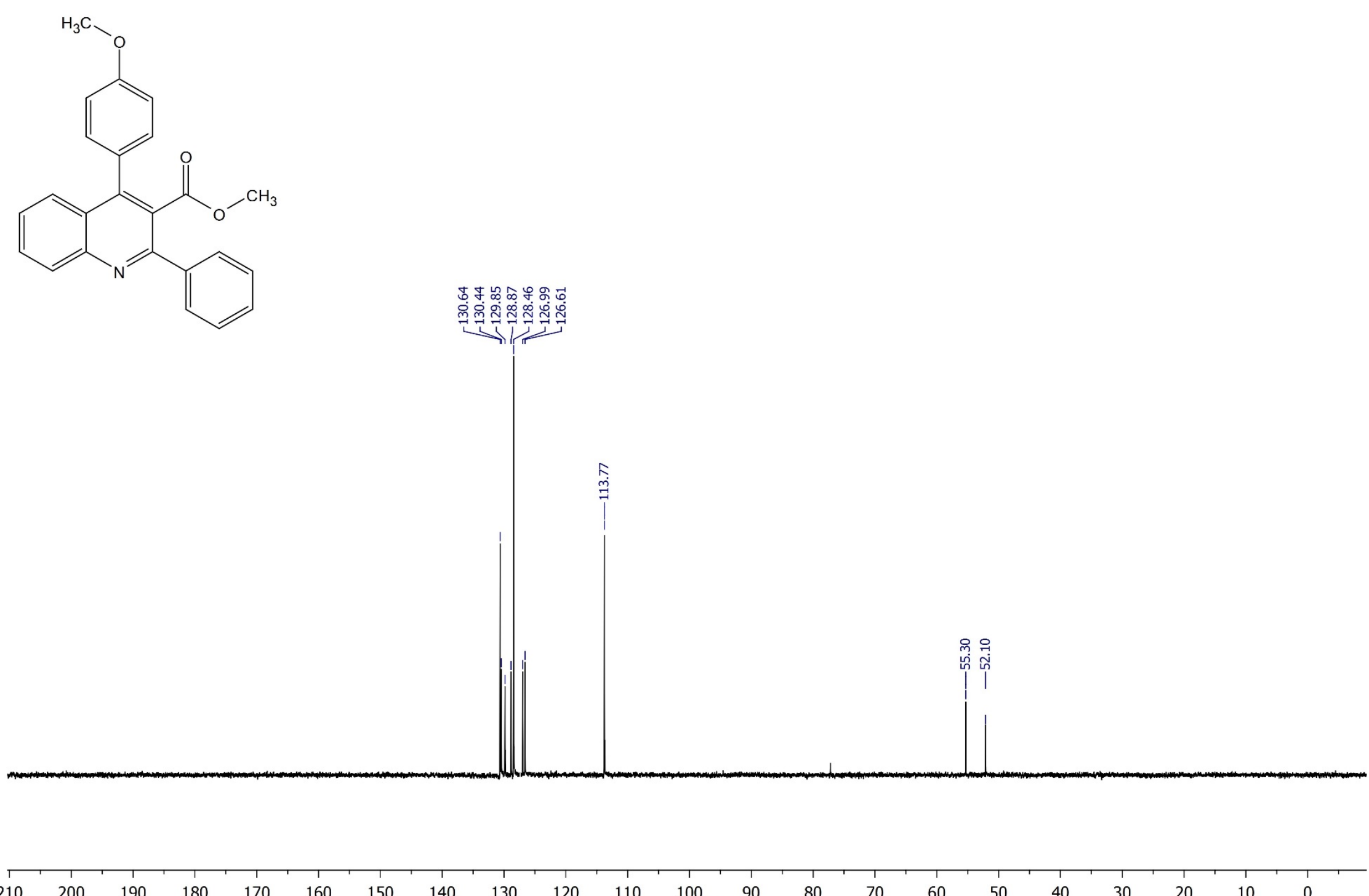

$150 \quad 140 \quad 130$

120

$110 \quad 100 \quad 90$

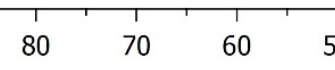

$50 \quad 40$

30

20

10 
Methyl 4-(4-nitrophenyl)-2-phenylbenzo[h]quinoline-3-carboxylate 10a, ${ }^{1} \mathrm{H} \mathrm{NMR}, 400 \mathrm{MHz}, \mathrm{CDCl}_{3}$
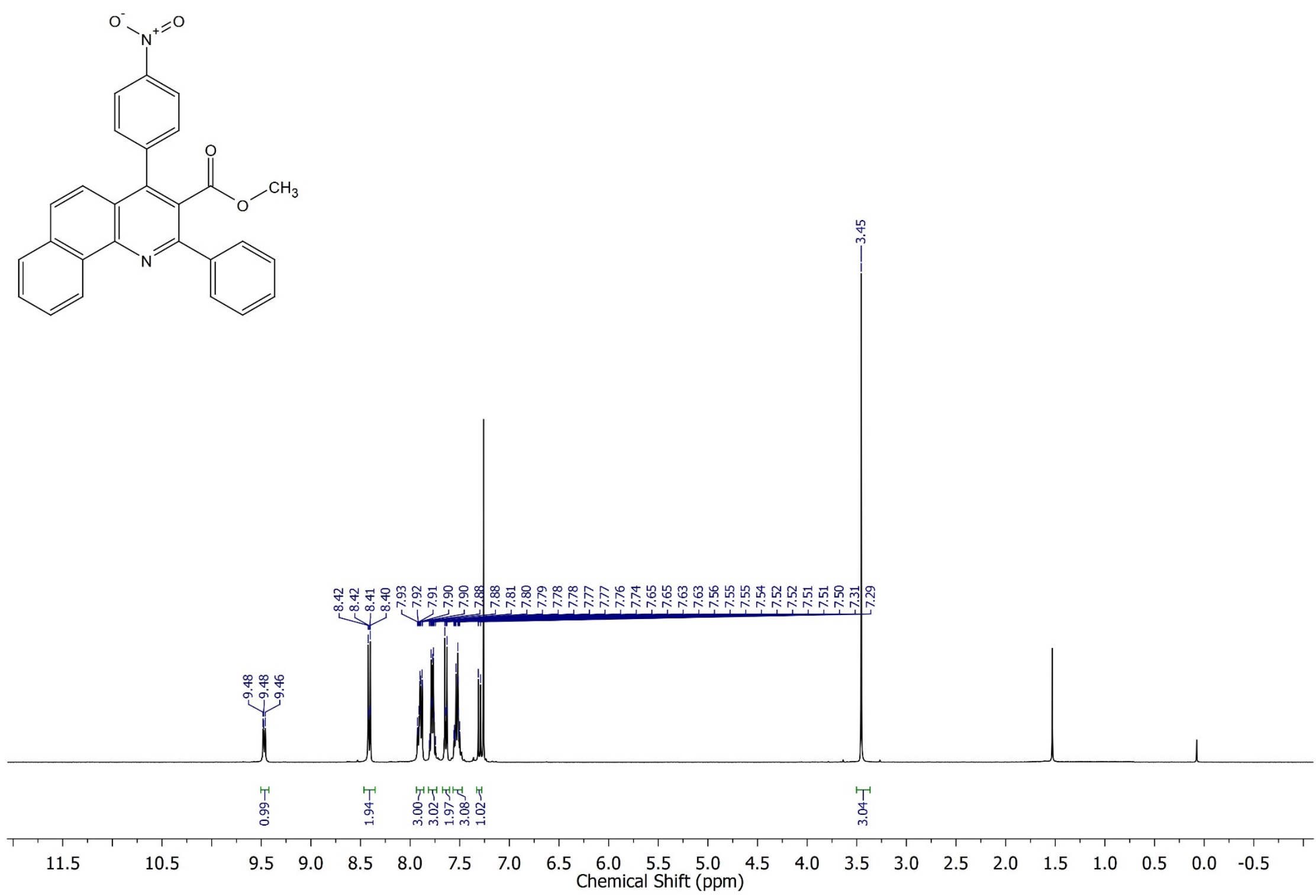
Methyl 4-(4-nitrophenyl)-2-phenylbenzo[h]quinoline-3-carboxylate 10a, ${ }^{13} \mathrm{C}\left\{{ }^{1} \mathrm{H}\right\} \mathrm{NMR}, 100 \mathrm{MHz}, \mathrm{CDCl}_{3}$
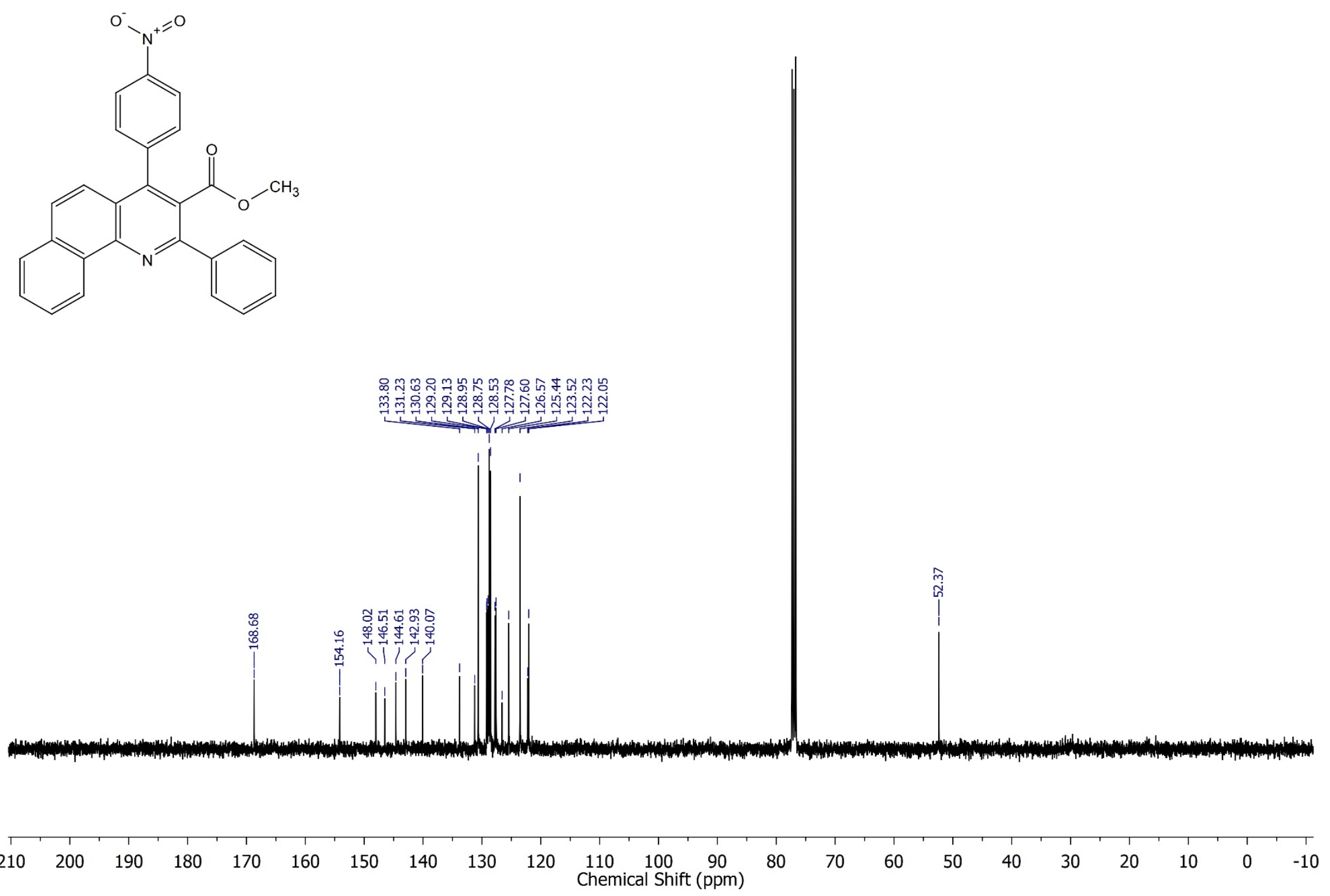
Methyl 4-(4-nitrophenyl)-2-phenylbenzo[h]quinoline-3-carboxylate 10a, DEPT, $100 \mathrm{MHz}, \mathrm{CDCl}_{3}$
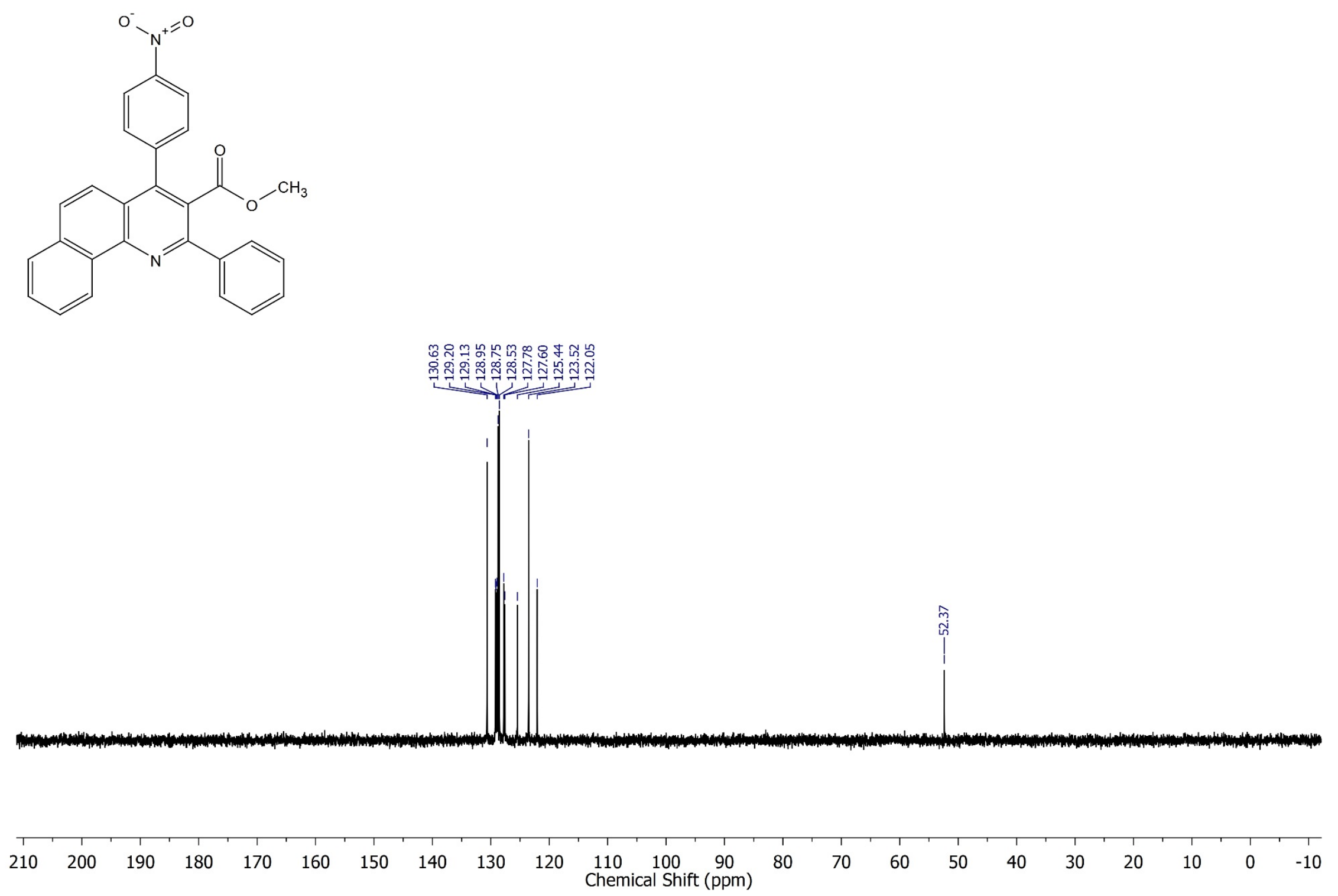
Methyl 4-(3,4-dimethoxyphenyl)-2-(thiophen-2-yl)benzo[h]quinoline-3-carboxylate 10b, ${ }^{1} \mathrm{H}$ NMR, $400 \mathrm{MHz} \mathrm{CDCl}_{3}$

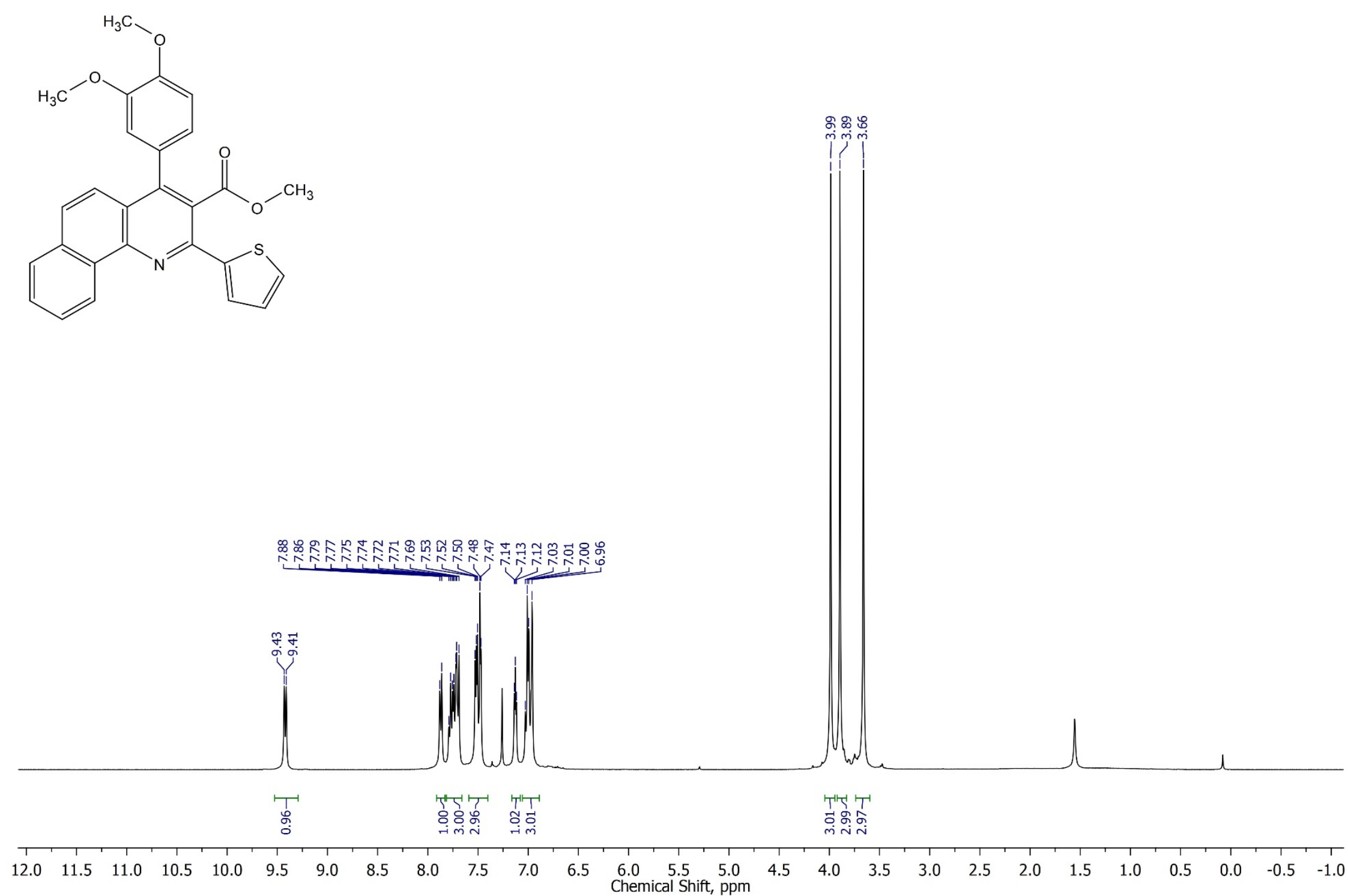


Methyl 4-(3,4-dimethoxyphenyl)-2-(thiophen-2-yl)benzo[h]quinoline-3-carboxylate $10 \mathrm{~b},{ }^{13} \mathrm{C}\left\{{ }^{1} \mathrm{H}\right\}$ NMR, $100 \mathrm{MHz} \mathrm{CDCl}_{3}$
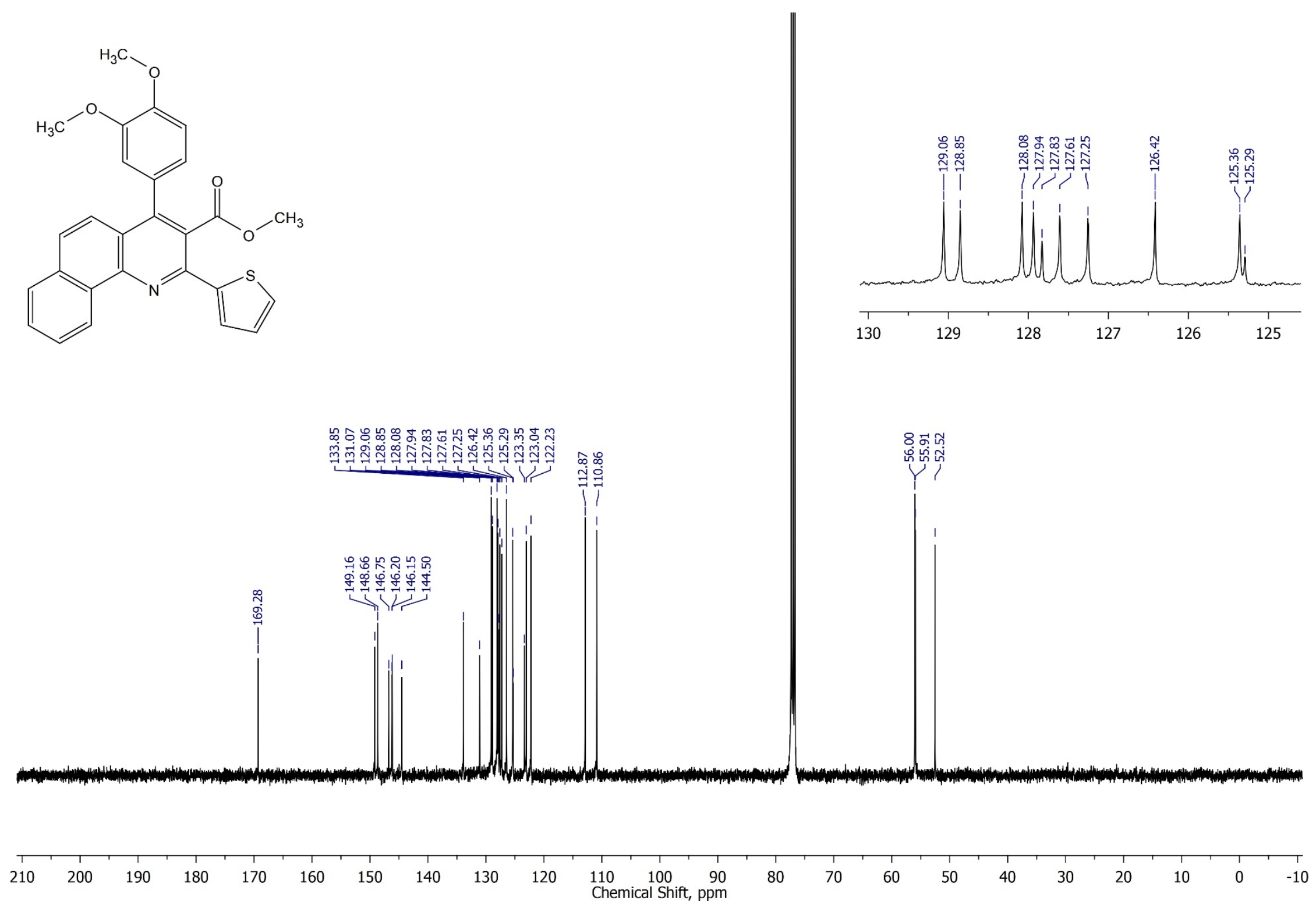
Methyl 4-(3,4-dimethoxyphenyl)-2-(thiophen-2-yl)benzo[h]quinoline-3-carboxylate 10b, DEPT, $100 \mathrm{MHz} \mathrm{CDCl}_{3}$
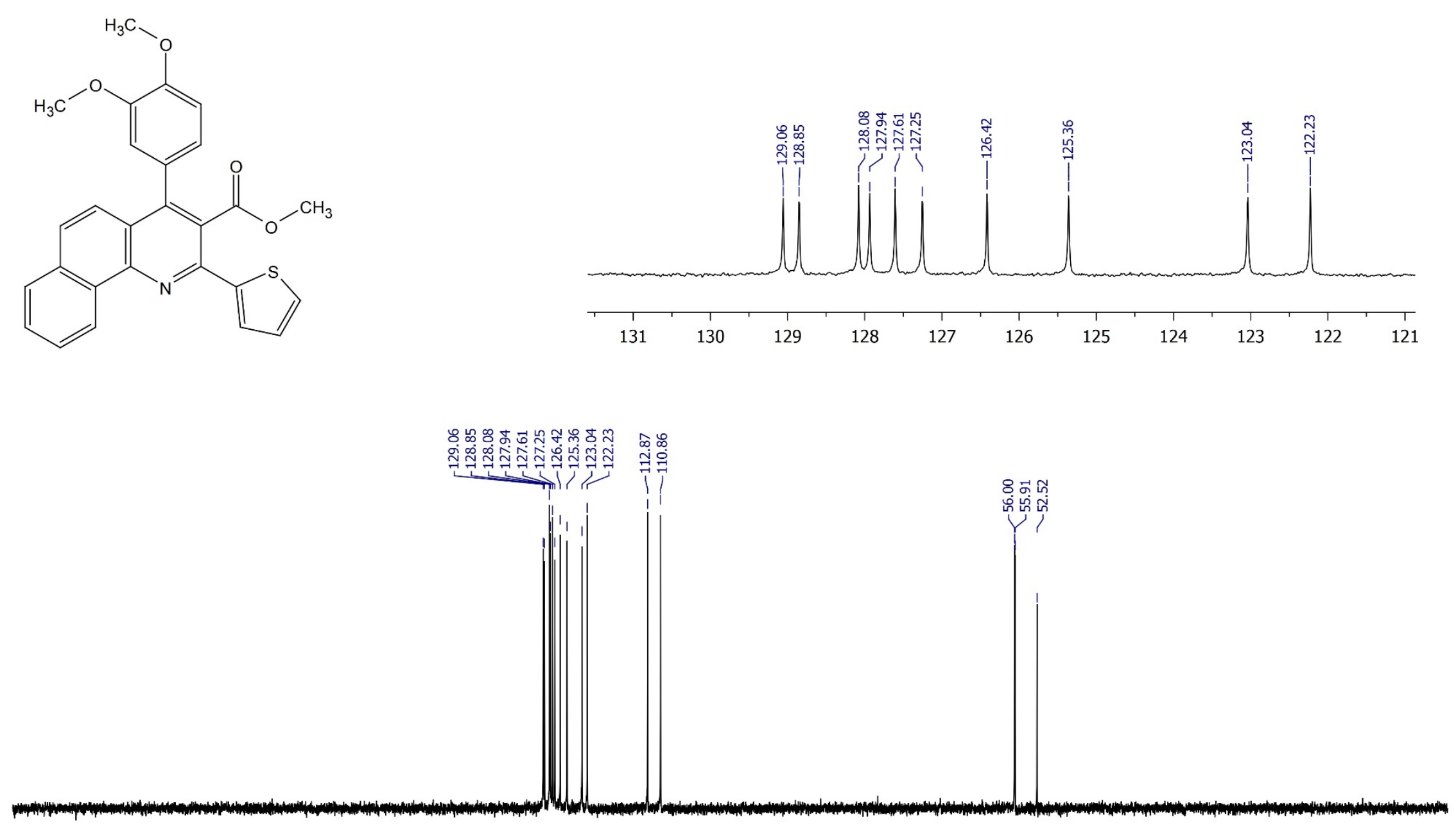

$10 \quad 200 \quad 190 \quad 180 \quad 170 \quad 160 \quad 150 \quad 140$

120

$\begin{array}{lr}110 & 100 \\ \text { Chemical Shift, } & 90\end{array}$

$80 \quad 70$

60

$50 \quad 40$

30

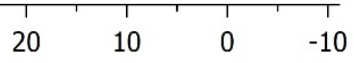

